On protein structure, function and modularity from an evolutionary perspective

\author{
Robert Pilstål
}

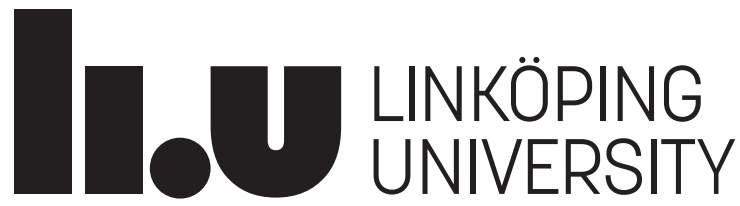

Linköping University

Department of Physics, Chemistry and Biology

Division of Bioinformatics

SE-581 83 Linköping, Sweden

Linköping 2018 
Edition 1:3

(C) Robert Pilstål, 2018

ISBN 978-91-7685-347-4

ISSN 0345-7524

URL http://urn.kb.se/resolve?urn=urn:nbn:se:liu:diva-147697

Published articles have been reprinted with permission from the respective copyright holder.

Typeset using $\mathrm{ET}_{\mathrm{E}} \mathrm{X}$

Printed by LiU-Tryck, Linköping 2018 
"Ei se kannatte."

- Meänkieli proverb 



\title{
POPULÄRVETENSKAPLIG SAMMANFATTNING
}

\begin{abstract}
Människan byggs upp av celler, de i sin tur består av än mindre beståndsdelar; livets molekyler. Dessa fungerar som mekaniska byggstenar, likt maskiner och robotar som sliter vid fabrikens band; envar utförandes en absolut nödvändig funktion för cellens, och hela kroppens, fortsatta överlevnad. De av livets molekyler som beskrivs centralt i den här avhandling är proteiner, vilka i sin tur består utav en lång kedja, med olika typer av länkar, som likt garn lindar upp sig i ett nystan av en (mer eller mindre...) bestämd struktur som avgör dess roll och funktion i cellen.
\end{abstract}

Intrinsiellt oordnade proteiner (IDP) går emot denna enkla åskådning; de är proteiner som saknar struktur och beter sig mer likt spaghetti i vatten än en maskin. IDP är ändå funktionella och bär på centrala roller i cellens maskineri; exempel är oncoproteinet c-Myc som agerar gaspedalför cellen - fel i c-Myc's funktion leder till att cellerna löper amok, delar sig hejdlöst och vi får cancer.

Man har upptäckt att c-Myc har en ombytlig struktur vi inte kan se; studier av punktvisa förändringar, mutationer, i kedjan av byggstenar hos c-Myc visar att många länkar har viktiga roller i funktionen. Detta ger oss bättre förståelse om cancer men samtidigt är laboratoriearbetet både komplicerat och dyrt; här kan evolutionen vägleda oss och avslöja hemligheterna snabbare.

Molekylär evolution studeras genom att beräkna variation i proteinkedjan mellan besläktade arter som finns lagrade i databaser; detta visar snabbt, via nätverksanalys och grafteori, vilka delar av proteinet som är centrala och kopplade till varandra av nödvändighet för artens fortlevnad. På så vis hjälper evolutionen oss att förstå proteinfunktioner via modeller baserade på proteinernas interaktioner snarare än deras struktur.

Samma modeller kan nyttjas för att förstå dynamiska förlopp och skillnader mellan normala och patologiska varianter av proteiner; mutationer kan uppstå i vår arvsmassa som kan leda till sjukdom. Genom analys av proteinernas kopplingsnätverk i grafmodellerna kan man bättre förutsäga vilka mutationer som är farligare än andra. Dessutom har det visat sig att en sådan representation kan ge bättre förståelse för den normala funktionen hos ett protein än vad en proteinstruktur kan.

Här introduceras även konceptet proteinprimärer, vilket är en abstrakt representation av proteiner centrerad på deras interaktiva mönster, snarare än på partikulär form och struktur. Det är en förhoppning att en sådan representation skall förenkla diskussionen anbelangande proteinfunktion så till den grad att strukturbestämmelse av proteiner, som är en mycket kostsam och tidskrävande process, till viss mån kan anses vara sekundär i betydelse jämfört med funktionellt modellerande baserat på evolutionära data extraherade ur våra sekvensdatabaser. 
We are compounded entities, given life by a complex molecular machinery. When studying these molecules we have to make sense of a diverse set of dynamical nanostructures with wast and intricate patterns of interactions. Protein polymers is one of the major groups of building blocks of such nanostructures which fold up into more or less distinct three dimensional structures. Due to their shape, dynamics and chemical properties proteins are able to perform a plethora of specific functions essential to all known cellular lifeforms.

The connection between protein sequence, translated into protein structure and in the continuation into protein function is well accepted but poorly understood. Malfunction in the process of protein folding is known to be implicated in natural aging, cancer and degenerative diseases such as Alzheimer's.

Protein folds are described hierarchically by structural ontologies such as SCOP, CATH and Pfam all which has yet to succeed in deciphering the natural language of protein function. These paradigmatic views centered on protein structure fail to describe more mutable entities, such as intrinsically disordered proteins (IDPs) which lack a clear defined structure.

As of 2012, about two thirds of cancer patients was predicted to survive past 5 years of diagnosis. Despite this, about a third do not survive and numerous of successfully treated patients suffer from secondary conditions due to chemotherapy, surgery and the like. In order to handle cancer more efficiently we have to better understand the underlying molecular mechanisms.

Elusive to standard methods of investigation, IDPs have a central role in pathology; dysfunction in IDPs are key factors in cellular system failures such as cancer, as many IDPs are hub regulators for major cell functions. These IDPs carry short conserved functional boxes, that are not described by known ontologies, which suggests the existence of a smaller entity. In an investigation of a pair of such boxes of c-MYC, a plausible structural model of its interacting with Pin1 emerged, but such a model still leaves the observer with a puzzle of understanding the actual function of that interaction.

If the protein is represented as a graph and modeled as the interaction patterns instead of as a structural entity, another picture emerges. As a graph, there is a parable from that of the boxes of IDPs, to that of sectors of allosterically connected residues and the theory of foldons and folding units. Such a description is also useful in deciphering the implications of specific mutations.

In order to render a functional description feasible for both structured and disordered proteins, there is a need of a model separate from form and structure. Realized as protein primes, patterns of interaction, which has a specific function that can be defined as prime interactions and context. With function defined as interactions, it might be possible that the discussion of proteins and their mechanisms is thereby simplified to the point rendering protein structural determination merely supplementary to understanding protein function. 


\section{Acknowledgments}

Ett stort tack till mina handledare för all den tid och energi ni har investerat i mig. Särskilt tack till Björn för lärdomen om värdet $i$ ordning och Maria för nyttan och nöjet med mycket oordning!

The group members of Maria's cove has been part of the majority of the works I've been involved with; special thanks to Madhan for his collaborative efforts and funny jokes in the lab, all the way from undergraduate to England. Thanks for your share of work Meri on the projects. Tack för handledningen i labbet och samarbetet i artikeln Sara! För att inte glömma alla glada stunder med Amelie, Cecilia och er andra som kommit och gått under Sunnerhagens tak!

Furthermore, I shall not forget the plentiful moments of discussion with Claudio, Malin nor Sankar, in the Wallner laboratory, with regard to anything from protein to human interactions. Hoppas du får en minst lika lärdomsfull resa Isak!

Forum Scientium skall tackas för alla de trevliga stunderna, inspirerande diskussioner och föreläsningar jag därigenom fått delta i. Speciellt tack går till Stefan, Anette och Charlotte för att de håller dörrarna öppna för fler generationer av Forum-medlemmar! Utan detta forum hade inte Martin och Andrey kunnat elda på tankarna runt evolution, eller jag fått utflöde för organiserande i samarbetet runt sommarkonferensen med Jonas; ett mycket trevligt arbete som jag är tacksam för! But there's more, so much more; Anna, Ankit, Bela, Camilla, Christopher, Fredrik, Josefin, Jesper 11 Judit, Karin, Kjersti, Leffe, Lingyin 2 Niclas, Patricia, Sofie, William et al. thanks for all the laughs!

To Rosalie and your team; thanks for you hospitality, the joint efforts in publications and discussions on the MYC paper and related matters!

Jill, thank you for your invaluable feedback and joint effort!

Mika, tack för dina kommentarer och tankar; det har varit roligt att jobba med dig även om det var ringa tid och än inte har kommit i hamn.

Detsamma gäller dig Berkant; det har varit inspirerande att få prata med någon från ett helt annat fält och få inblick i hur saker kan göras där - tack för din tid och gemytliga energi!

Joel, det har varit otroligt schysst av dig att raffla in som extra-mentor här och där; för mig har det inneburit välbehövlig feedback och verklighetskoll i stunder av grövre förvirrning - Tack för detta, och jag hoppas vi snubblar in i varandra även framöver!

\footnotetext{
${ }^{1}$ Du får fixa det där garaget...

${ }^{2}$ Let me know when you're having the next hotpot!
} 
Thomas, som mentor har du varit professionell och framstått mycket pragmatisk och öppenhjärtad de gångerna som vi faktiskt mötts; om jag hade känt dig så tidigare hade nog dessa tillfällen blivit fler i antalet! Tack för din tid.

Thanks to IFM for your time and efforts to put up with me the last 5 years; I do appreciate the moments of fun I've had with you guys in the chem-corridor, even if they might seem few and long paces apart; but that, I guess, is just my own impression. Thank you Peter, Johan, Magdalena and all of the rest of you!

Tack Magnus för din kurs i kaotisk teori; den var minst sagt upplysande, hoppas du hinner hålla den för många generationer till!

SBW2016 was a hit, and it was thanks to the awesome group of troopers leading the work to a successful delivery, that resulted in a top-notch international scientific forum; Emil, Niclas, Andrey, William, Claudio and Andreas - Great job!

Sedan vill jag tacka Forsmarks skola, särskilt Pudas, Olle och Madis; utan er hade jag inte funnit den inspiration som ledde till min utbildning och senare denna doktorsavhandling! Dessutom är det på sin plats att tacka er forsmarkare som jag känt och känner - ni har för alltid en plats i mitt hjärtd $\Omega^{3}$ jag glömmer er inte.

För er värme, omtanke och generositet vill jag tacka Maria, Rune, Tomas \& Thomas, Hans, Carolina, Rozalyn, Magdalena, Emma och alla er andra som kommer och går under sanghans tak; de senaste två åren hade inte varit möjliga utan er samlade vänlighet!

Johan, du ställer upp i sol och storm, varför vet jag egentligen inte - men jag är ändlöst tacksam; utan dig hade jag inte kunnat puttra runt i en miljövidrig dieselbil alla dessa åren! Men du får bli bättre på att casha in på gentjänsterna, så ses man oftare :)

Mormor, mina mostrar, morbröder och farbror Nisse; utan er hade jag inte tagit mig hit heller - tack för att ni ställde upp när jag verkligen behövde det, och visade att man kan ge utan att behöva ta!

Jag reserverar även ett tack här för mina föräldrar; då jag inte idag förstår vad jag har att tacka för, så antar jag att denna okunskap enbart kommer ur min i dagsläget begränsade insikt. Därför riktar jag ett varmt och fullhjärtat tack bona fides ${ }^{4}$ till er båda, för allt. Det är ju inte det enklaste att vara förälder, har jag märkt... :)

Tack Brorsan, helt enkelt för att du finns; vi får ta den dära fisketuren vi pratat om - nu när jag kommer ha all tid i världen att göra vad jag vill ;)

Sist och inte minst, vill jag tacka Elisabeth och min lilla Mån§5 ${ }^{5}$ För oss finns det tyvärr inte nog med ord för att ge en fullkomlig rättvisa åt min uppskattning till er; vad jag än mäktar skriva, ter sig torftigt i motsvarighet mot denna helhet. Nu undrar jag bara; var ska vi gräva nästa rabatt?

\footnotetext{
${ }^{3}$ Och en plats på min kavaj.

${ }^{4}$ Att en framtida inkarnation av mig själv ska ha kommit till insikt.

${ }^{5}$ Som då faktiskt också är minst i sammanhanget...
} 


\section{Author Contributions}

\section{Published Papers}

I Sara Helander, Meri Montecchio, Robert Pilstål, Yulong Su, Jacob Kuruvilla, Malin Elvén, Javed M.E. Ziauddin, Madhanagopal Anandapadamanaban, Susana Cristobal, Patrik Lundström, Rosalie C. Sears, Björn Wallner, and Maria Sunnerhagen. "PreAnchoring of Pin1 to Unphosphorylated c-Myc in a Fuzzy Complex Regulates c-Myc Activity". en. In: Structure 23.12 (Dec. 2015), pp. 2267-2279. ISSN: 09692126. DOI: 10.1016/j.str.2015.10.010 URL: http://linkinghub.elsevier.com/ retrieve/pii/S0969212615004499 (visited on 02/01/2016)

CONTRIBUTION: As joint first author, most work pertaining to computational modelling and analysis. Wrote related parts of the manuscript, participated in the writing of the full paper, and actively participated in submission and journal communication

II Madhanagopal Anandapadamanaban, Robert Pilstål, Cecilia Andresen, Jill Trewhella, Martin Moche, Björn Wallner, and Maria Sunnerhagen. "Mutation-Induced Population Shift in the MexR Conformational Ensemble Disengages DNA Binding: A Novel Mechanism for MarR Family Derepression". en. In: Structure 24.8 (Aug. 2016), pp. 1311-1321. ISSN: 09692126. DOI: $10.1016 / \mathrm{j} \cdot \mathrm{str} .2016 .06 .008$ URL: http://linkinghub.elsevier.com/retrieve/pii/s0969212616301332 (visited on 04/11/2017)

CONTRIBUTION: As joint first author, extensive contribution to analysis of simulation data and graph modelling. Wrote related parts of the manuscript, participated in the writing of the full paper, and actively participated in submission and journal communication

III Arne Elofsson, Keehyoung Joo, Chen Keasar, Jooyoung Lee, Ali H. A. Maghrabi, Balachandran Manavalan, Liam J. McGuffin, David Ménendez Hurtado, Claudio Mirabello, Robert Pilstål, Tomer Sidi, Karolis Uziela, and Björn Wallner. "Methods for estimation of model accuracy in CASP12". eng. In: Proteins (Oct. 2017). ISSN: 1097-0134. DOI: 10.1002 /prot.25395

CONTRIBUTION: RP created the automatic domain partitioner that figured in the method employed by the Wallner group. It is further detailed in [109]. 


\section{Manuscripts}

I Robert Pilstål and Björn Wallner. "Improvements in Protein Model Quality Assessment from Automated Domain Partitioning using Spectral Clustering". Apr. 2018

CONTRIBUTION: RP and BW designed the project. RP executed study and produced figures, collated material and outlined manuscript. BW rewrote the manuscript.

III Robert Pilstål, Maria Sunnerhagen, and Björn Wallner. "Functional Interaction Network of c-MYC Conserved Regions determined by Evolutionary Couplings". Apr. 2018

CONTRIBUTION: RP took part in conceiving and designing the project. RP contributed the initial work, produced figures, collated material and outlined manuscript. BW rewrote the manuscript.

\section{Not Included in Thesis}

IV William B. Tu, Sara Helander, Robert Pilstål, K. Ashley Hickman, Corey Lourenco, Igor Jurisica, Brian Raught, Björn Wallner, Maria Sunnerhagen, and Linda Z. Penn. "Myc and its interactors take shape". en. In: Biochimica et Biophysica Acta (BBA) Gene Regulatory Mechanisms 1849.5 (May 2015), pp. 469-483. ISSN: 18749399. DOI: $10.1016 / j . b b a g r m .2014 .06 .002$ URL: http://linkinghub.elsevier. com/retrieve/pii/S1874939914001540

REASON: Review article. 


\section{Contents}

Abstract v v

Acknowledgments viii

\begin{tabular}{|l|l}
\hline Author Contributions & ix
\end{tabular}

Contents $\quad x i$

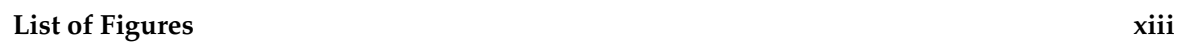

\begin{tabular}{ll}
\hline Abbreviations & 1
\end{tabular}

\begin{tabular}{ll|l}
\hline & Introduction & 3 \\
\hline
\end{tabular}

$1.1 \quad$ What is Science? . . . . . . . . . . . . . . . . . . . . . 3

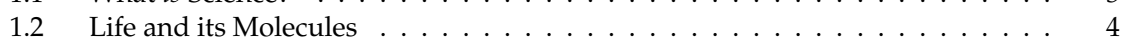

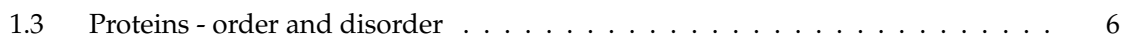

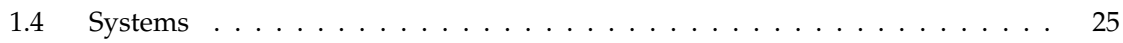

\begin{tabular}{|lll}
\hline 2 & Thesis & 27
\end{tabular}

\begin{tabular}{|l|l|}
\hline 3 Method & 29 \\
\hline & 3.1
\end{tabular}

$3.1 \quad$ Alignments . . . . . . . . . . . . . . . . . . . . . . . . . . . . . . . . . . . . . 29

$3.2 \quad$ Contact prediction $\ldots \ldots \ldots \ldots \ldots \ldots \ldots$

$3.3 \quad$ Structure prediction $\ldots \ldots \ldots \ldots$. . . . . . . . . . . . . . . . . . . . . . . . 38

3.4 Dynamics simulation. . . . . . . . . . . . . . . . . . . . . . . . . 40

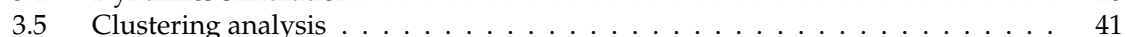

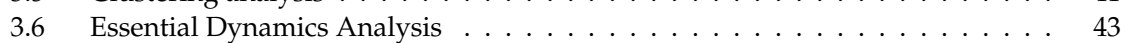

$3.7 \quad$ Graphs and Structures $\ldots \ldots \ldots \ldots \ldots$. . . . . . . . . . . . . . . . . . . . . 45

$3.8 \quad$ Allosteric Network Analysis $\ldots \ldots \ldots$. . . . . . . . . . . . . . . 47

3.9 Ensemble Quality Assessment . . . . . . . . . . . . . . . . . . . . . 47

4 Present Investigations $\quad 49$

\begin{tabular}{|lll}
4.1 & Function in transiently ordered islets of intrinsically disordered proteins (Paper I) & 49
\end{tabular}

$4.2 \quad$ Functional analysis of protein structure dynamics using graph models and mul-

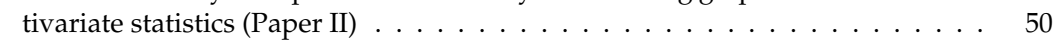

4.3 Improvements in Protein Model Quality Assessment from Automated Domain Partitioning using Spectral Clustering (Paper III and Manuscript I) $\ldots \ldots . \quad 50$

4.4 Functional Interaction Network of c-MYC Conserved Regions determined by

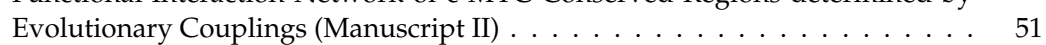

\begin{tabular}{ll|l}
5 & Conclusion & 53
\end{tabular} 
$6 \quad$ Future Perspectives

\begin{tabular}{|ll}
\hline Bibliography & 57
\end{tabular}

\begin{tabular}{ll}
\hline Paper I & 81
\end{tabular}

\begin{tabular}{ll}
\hline Paper II & 117
\end{tabular}

\begin{tabular}{ll}
\hline Paper III & 141
\end{tabular}

\begin{tabular}{ll}
\hline Manuscript I & 157
\end{tabular}

\begin{tabular}{ll}
\hline Manuscript II & 179
\end{tabular} 


\section{List of Figures}

1.1 Illustration of how the concept of human relates to its organs, the tissue of the organs to the cells and as the organelles of the cells relate to the proteins constituting them in turn. $\ldots \ldots \ldots \ldots \ldots \ldots \ldots \ldots$

1.2 Illustration on the central dogma of genetics. [1 $\mid$ (a) The genome is transcribed (b) into mRNA, which is translated (c) at the ribosome into a peptide sequence, which in turn folds into a structure that carries a certain function.

I.3 $\quad$ Illustrating the classic idea that a protein sequence folds through a series of steps into a compact native structure. In a more modern view, there is actually an ensemble of series within which the folding can follow many routes ending up into an folded state that is constantly transforming through the states of a whole ensemble of native structures. [49| The example shown here is that of the 35 residues long headpiece domain of Villin [32 $\mid$ available as PDB entry 1WY3 available at www.rscb.org [25]. The protein was rendered using the Open Source community version of the software PyMOL. [38]|

1.4 Illustrating the RRI through DDI to PPI conceptual hierarchy. Indicates that network interaction walks can be interpreted as functions, mechanisms and pathways

\begin{tabular}{|l} 
on the corresponding levels. $\ldots \ldots \ldots \ldots \ldots$ \\
\hline $1.5 \quad$ A simplified illustration of the sequence when a protein folds on the ribosome. The
\end{tabular} protein domain architecture concepts of folding units |80], foldons [49], sectors |62], primes and constellations are indicated by red in the hierarchy. Primes and constellations provide a model for all levels of the hierarchy as they focus on the interaction network, supporting a fully modular view of protein structure centered on a proteins functional aspects. $\ldots \ldots \ldots \ldots \ldots \ldots$

1.6 Protein primes are best realized as clusters of nodes in network models. They are here depicted as nodes with one color per prime, and their interactions indicated by edges. Distances does not signify real distance, as it is just a sorting; it is the connections that signify functional relationships. (a) There are prime constellations consisting of primes carrying simple functions that are not constellations themselves. They form clique-like structures in the constellation with weaker interactive patterns between them than within. The more primes in the constellation, the more complex it is. (b) Some functions are expected to be larger than other primes, here three are depicted from the bottom up in growing order. Just as with prime numbers, it is expected that there could be functions that are increasingly large but cannot be split up in smaller well defined primes. The minimum size of primes needs to be determined from a view of utility, which will have to be evaluated with respect to some assumptions (i.e. axioms). (c) Protein primes are characterized by their internal and external interactions only. Here, two analogous primes are illustrated, though without any indication of external relations. $\ldots \ldots \ldots \ldots$ 
1.7 A bound sub-ensemble model of the c-MYC IDPs [134] MoRFs-like|136| MB0 in fuzzy complex |131| with PIN1|66|. Tryptophan 35 in PIN1 is indicated with red spheres, PIN1 bound $\mathrm{PO}_{4}$ with yellow spheres and the black arrow indicates the $\mathrm{N}$ to $\mathrm{C}$-terminal direction of the sequence of $\mathrm{c}-\mathrm{MYC}_{\mathrm{MB} 0} \mid \ldots \ldots \ldots \ldots$

3.1 Co-evolution, co-adaptation, evolutionary couplings and direct contacts are all gauged as variants of analysis on co-occurrence of amino acid types in columns of multiple sequence alignments. Here, a snipplet of an MSA is shown for illustrative purposes, highlighting two selected pairs of columns. These can be represented as vectors in $|\mathbb{Z}|_{<q}^{N}$ space, by encoding each character in the column as a number from 0 to $q-1$, for $q$ different kinds of amino acids in the amino acid alphabet. A weight is often attributed to each sequence sample $\left(m_{i}\right.$ 's to the right) which reflects how prevalent/conserved the sequence is in the protein family.

3.2 One hot expansion of the coordinate space into more dimensions, reducing the encoding to binary entries. (a) The residues are expanded into residue acids (b) which is an abstraction of the encoding of the data vectors into a matrix space. This forces all possible correlations to be linear or non-existent. (c) Visualization of the one-hot encoding of one residue column in the MSA as $q$ columns of binary vectors, out of which only one entry is non-zero per row. This expands the actual matrix (S) into a 4 -tensor, but in the implementations this is usually represented as a block matrix.

3.3 Simple example of one aspect of non-linear complexities arising from different environments and interactions surrounding the involved residues in any coupling. (a) A residue pair in a hydrophobic environment is expected to have one pattern of covariation, whilst a pair involved in catalysis on the surface of the protein $(\mathbf{b})$ is expected to have another. Thus if both are expressed over the same dimensions with $q$ states each, there is no single way of sorting the amino acids for both correlations to become linear. .

3.4 Illustration of mutations of a protein system constituted out of one protein highlighting a particular internal interface essential for proper function. (I) At first the protein is in a stable state, thanks to its amino acid composition. During an extended period of relaxation, when the protein system is not needed for the survival of individuals carrying it, a number of mutations accumulate within the population generating variant strains (II, III and IV). When the protein function is suddenly required due to a selection event, the two strains that have a faulty interface perishes (II and III), leaving us with only the two strains carrying intact interfaces to be observed in the present time (I and IV). When querying our sequence databases for homologous sequences, with either the sequence pertaining to I, IV or some other related sequence, we will thus only find I and IV, not the sequences II or III since they have gone extinct and are not present in the databases. This bias is caused by the fact that the content of our current day sequence databases reflect the currently available fauna of earth, since it is here and on living organisms that we have performed most sequencing experiments. Thus, the combinations of amino acid patterns that we can observe for this particular interface becomes constrained to those of I and IV, leaving combinations II and III out of the mix. It is this process that leaves traces of allowed patterns of amino acid combinations in our sequence databases; patterns that can be detected using co-occurrence modeling.| .

Illustration of conceptual difference between global and local similarity measures. Global similarity (a) measures the difference between the two conformations as a whole, given an optimal superposition, whilst local similarity measures (b) focus on the differences between internal configuration in a superposition independent manner.

3.6 A sketch of a graph representation of a protein structure. The protein structures (a) residues are taken as the nodes with their distances as a parameter in calculating their edges. This is used in formulating the graph model (b), resulting in a matrix with the edges as entities and the node sequence along the rows and columns (c). . 


\section{Abbreviations}

c-MYC Myc proto-oncogene protein

CASP The Critical Assessment of protein Structure Prediction experiments

CATH CATH Protein Structure Classification database

CSP Chemical Shift Perturbation

DC Direct Contact

DDI Domain-Domain Interactions

DNA Deoxyribonucleic acid

EC Evolutionary Coupling

EDA Essential Dynamics Analysis

EM Electron Microscopy

GDT Global Distance Test

GO Gene Ontology

GROMACS Groningen Machine for Chemical Simulations

GPU Graphics Processing Unit

HNCO a Triple-resonance nuclear magnetic resonance spectroscopy sequence

HSQC Heteronuclear single quantum coherence spectroscopy

ID Intrinsically Disordered

IDP Intrinsically Disordered Protein

IDR Intrinsically Disordered Region

LDDT Local Distance Difference Test

LGA Local Global Alignment

MB0 MYC box 0, etc. 
MDR Multi-drug resistance

MexR Multidrug resistance operon repressor mexR

MoRFs Molecular recognition features

MSA Multiple Sequence Alignment

NMR Nuclear Magnetic Resonance

NSC National Supercomputer Centre at Linköping University

HMM Hidden Markov Model

PCA Principal Component Analysis

PDB Protein DataBank

PIN1 Peptidyl-prolyl cis-trans isomerase NIMA-interacting 1

PPI Protein-Protein Interactions

PSN Post-translational modification

PTM Post-translational modification

QA Quality assessment

RMSD Root-mean-square deviation of atomic positions

RNA Ribonucleic acid

RRI Residue-Residue Interactions

SCA Statistical Coupling Analysis

SCOP Structural Classification Of Proteins database

TM template modeling 


\section{Introduction}

\subsection{What is Science?}

"When the Philosophers speak of gold and silver, from which they extract their matter, are we to suppose that they refer to the vulgar gold and silver?

By no means; vulgar silver and gold are dead, while those of the Philosophers are full of life."

- Théodore Henri de Tschudi. Hermetic Catechism in his L'Etoile Flamboyant ou la Société des Franc-Maçons considerée sous tous les aspects. 1766. (A.E. Waite translation as found in The Hermetic and Alchemical Writings of Paracelsus.) (from Wikipedia)

Science, as understood by this thesis, is the formal cognitive modeling of the matter that we, beings of mind, percieve as the ether of reality that is forcibly observed by all the minds of the billions of lifeforms inhabiting our planet - and so, by extension, all of our known universe.

As such, science can never be understood as the ultimate wisdom of everything; albeit it can be the ultimate theory, any theory is limited to the fact that it is restrained to be a formal system; then as a corollary to Gödels incompleteness theorems, for any formal system, there will always be axioms which cannot be proved within the system itself - thus rendering the theory incomplete in its essence, no matter what.

Therefore any ultimate theory will have a bottomless depth, an infinite number of complexities, in order to fully describe every vanishingly small aspect of our infinite reality. As such, it is not within the scope of this thesis to produce any perfect models of the subject matter, but merely a humbling "as good as it gets, as of yet" depiction of our current understanding of the discussed aspects of reality. 
One might then ask what is the value of science if it cannot be absolute, but to be frank there is no value at all in science, and nor should it be. Whenever we start to attribute value to science, we are going into politics, technology and development, rather than pure science. Science as a factual thing, does not attribute measures of values, as these themselves are discussed as topics of science. In the scientific discussion of values as their abstraction in mathematical measure theory, one is on the square base of understanding that there are multiple measures for every one thing; depending on what you want to measure, that is what you define and argument for as proper value, there will be an appropriate measure of that value. Therefore any critiscism of science for its value, is not internal of science, but externally applied form the views of past, current or future utility, which is in itself a definition of public opinion and political whims. An illustrating case is the fate of the iconified Christopher Columbus.

In the beginning of the 16th century, Columbus returned with cacao and potato to Europe, for which he was later disgraced. According to his subjects, Columbus had committed vicious crimes against humanity and the crown judged him harshly for his poor ethical precepts. Just as Columbus had to promise wealth and good governance in order to be granted the funds that enabled him to settle his appetite for exploration 1 so does scientists have to promise results and adhere to ethical principles of morality in order for funding agencies to promote their endeavors. Yet, the lack of measurable results as gauged by the concurrent society might not always mean that the scientific enterprise itself carried no fruit with it.

The author of this thesis however is no Christopher Columbus, nor is he particularly interesting in any other way. Merely on the parable of embarking on a dream of discovery, then this thesis is the authors potatoes and tortured committee.

\subsection{Life and its Molecules}

"Snus och Enzymer, det är livet." - Olle Östling

\section{A human comes into being}

We commonly accept that we have one mind and one body, each separate from any other persons body. These concepts can be further divided, as we have observed through centuries of scientific investigation, realizing that our bodies themselves are built up by different compartments and structures that we term organs, which are lumped together and held in place by our skin $[1.1$.

These organs that build our bodies have different faculties that they use to perform particular functions that help perpetuate the cycle of happenings that we identify as life, but they are themselves built up by different chemo-mechanical parts. These smaller parts, be it blood vessels or other structural linings separating compartmets from each other, are also built up by even smaller structures. Virtually all structures in our bodies are constituted out of cells, small more or less self regulating and sustaining units of living tissue, which in their bare essense is constitued by a small compartment of intra-cellular fluid called cytosol, separated by a cell membrane form the outer world.

The cells of a human body pertain to the major phylogenia eukaryotes, which is the term used to distinguish cells that have an extra special compartment known as the cell nucleus, within which its genes are stored. In contrast, other phylogenia such as the prokaryotes lack this compartment and have their genes free-floating within their cytosol, or cytoplasm.

These eukaryotic cells own more pecularities, more resembling the abstract notion of structure that we have attributed to the human body as a whole, as they also contain structures known as

\footnotetext{
${ }^{1}$ While not torturing his fellow men.
} 


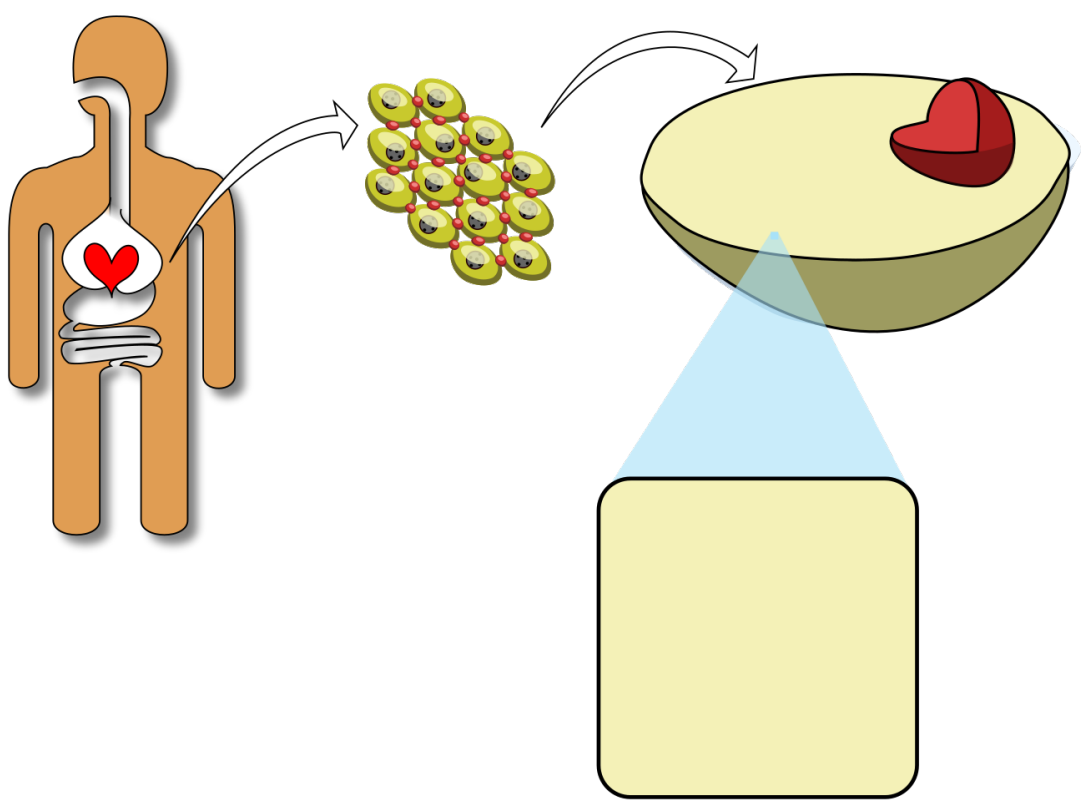

Figure 1.1: Illustration of how the concept of human relates to its organs, the tissue of the organs to the cells and as the organelles of the cells relate to the proteins constituting them in turn.

organelles. Organelles are to cells what organs are to the human body; they perform specialized tasks, and aid in separating different kinds of molecular systems from others, in order to keep them properly and efficiently functioning. For an example complex building material production is situated at the porous nuclerar membrane and in separate extra compartments known as the $E R$ and Gogli, while digestion is handled in compartments originating from the outer cellular membrane known as lysosomes.

All of these different compartments and their characteristics are maintained and defined by the interactions of a vastly complex molecular machinery that is constantly replicating and perpetuating itself.

\section{The Vitae Emergent}

The intricacy of the self replicating machinery that we call life sustains characteristics that we can term as emergent. [13] Thus as a whole, a life process is an emergent of the analytical components that constitutes it. That is, no where in any singular component of this machinery can we find something that is different to any (relatively) inanimate object, such as a pebble or a stone, yet as a whole it perceives, decides, takes action and moves.

This emergent description of life inevitably then brings the age old alchemical concept of vitae [31] to mind; i.e. the idea that animate objects and systems carry a certian vital energy which inanimate objects and systems lack. [15] 


\section{INTRODUCTION}

In taking vitalism on the interpretation as the emergent, we will discover that it encompasses the abstract notions of what a native structure, protein dynamics and protein interaction networks actually are to life as a process. By looking on these concepts from the vitae emergent point of view, one see that all of these abstractions constitute different aspects of such an emergent interpretation of vitae.

Nevertheless one should take extra caution in doing a contemporary comparison to such an old object of philosophy, since many factual propositions has been made in error under the umbrella of the term over the centuries it has been discussed. A simile can be seen on the comparison of the modern day field of epigenetics to that of the ideas of Lamarck and Lysenko.

Now, as far as the scientific discussion on the neo-Lamarckian or Lysenkov interpretation of the abstraction of epigenetics goes, there are those that want to retribute some of these aspects to ideas pushed forth by the earlier thinkers [124] while other detest that these ideas can even be attributed to their original publishers [106]. Of course, such detestation can be raised against all historic ideas; in such a light, even the theory of general relativity cannot be fully attributed to Einstein, since it necessarily builds upon the Galilean idea of relativity, the electromagnetic concept of light pushed by Planck, the Lorenzian mathematical transformations and the MichelsenMorley's concept of the invariability of the speed of light. [24] Although Einstein failed to cite his sources in his initial publications, it is to us evident what had inspired and enabled his legendary synthesis.

By keeping in mind the idea of the vitae emergent, while reading this thesis and its depiction of protein interactions and dynamics, the reader will be equipped with a broader perspective and wider foundation for a greater appreciation of the grandeur of the mechanistic networks of life that this thesis just might ignite.

\subsection{Proteins - order and disorder}

"Words do not make a man understand. It takes the man to understand the words."

- Alan watts citing chinese poem

When the very first protein structure was discovered in 1958 [72] it began to dawn on the scientific community that there would have to be a considerable effort invested in order to understand the interactions and functions of all proteins constituting such complex organisms as man. Since then numerous approaches to catalog and discriminate structures from each other has been developed, attributing function to the structures and inferring the function on their protein homologs. As will be discussed in the coming sections, protein structures are smaller building blocks in a whole, where the protein function cannot be understood without its context.

\section{Structural hierarchy}

Protein molecules are essentially long chains of smaller constituents known as amino acids, synthesized inside the cell at the ribosomes after being translated into messenger RNA from the DNA in the cell nucleus (Figure 1.2). These chains of amino acids coil up into three dimensional structures (Figure 1.3 which enable them to perform specific functions through interactions with other proteins and molecules, following the central dogma of Genomics. [1] This folding process follows another dogma coined by Anfinsen's [12], which postulates the thermodynamic hypothesis that the resulting structure is determined as the conformation with the lowest Gibbs free energy with respect to the proteins sequence and milieu.

In a protein chain, the amino acids are joined together by peptide bonds, forming a sequence with unique chemical properties known as the primary structure of the protein. The chains 


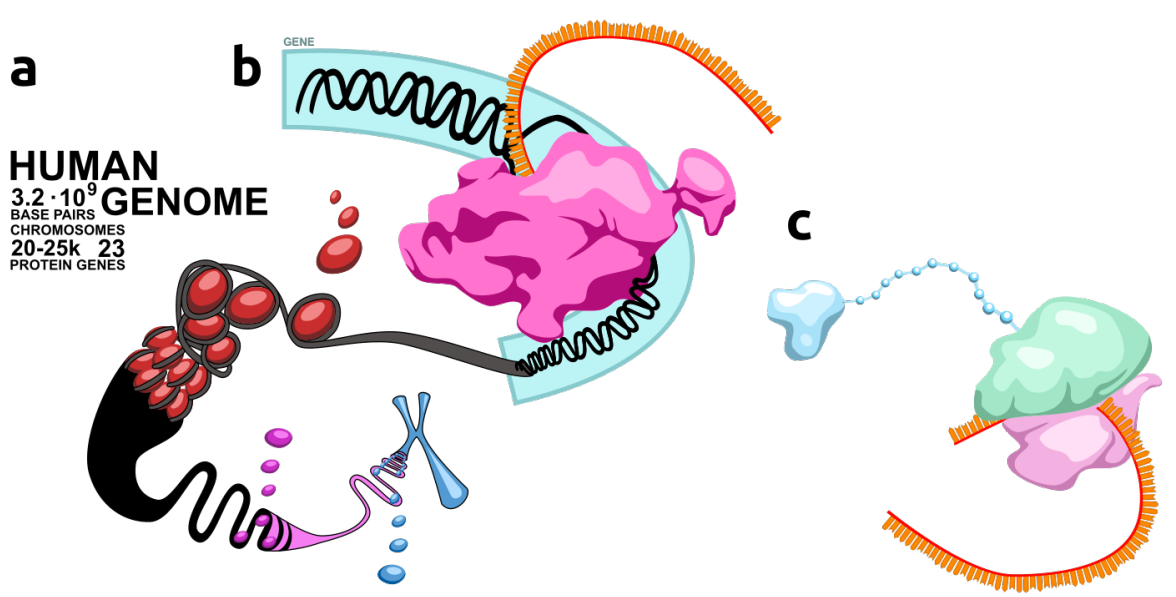

Figure 1.2: Illustration on the central dogma of genetics. [1] (a) The genome is transcribed (b) into mRNA, which is translated (c) at the ribosome into a peptide sequence, which in turn folds into a structure that carries a certain function.

chemical properties varies along the chain due to the different characteristics of each individual amino acid. These variations are determined largely by the side chains of each individual amino acid. Normally, there are 20 different possible side chains used in proteins in nature, giving the amino acids their unique properties.

As residues, the amino acids, start to interact, the backbone of the chain crinkle and coil up forming characteristic features known as secondary structure. There are three main classes of secondary structure; the helices, strands and coils.

The most prevalent secondary structure elements that forms are the alpha helices and beta strands. Alpha helices manifests as a cork-screw like coiling of the backbone that forms a complete turn around the longitudinal axis each 4-5 residues along the sequence. Beta strands are extended crinkled formations that make the amino acids protrude their side chains in opposing directions, alternating along the sequence. Beta strands combine and form sheet-like structures called beta sheets, while alpha helices can form up into bundles.

Any type of secondary structure can interact with each other, forming more complex 3D structures known as tertiary structures. It is in the complexities of the tertiary structures wherein the notion of protein domain has its origins, but the nature of its definition depends on from which perspective the protein is appreciated.

Quaternary structure describes how multiple copies of a protein chains can combine into even larger structures, such as homodimers, -trimers or larger constructs. Heterogeneous combinations stemming from different kinds of protein chains are also observed.

There exists an even higher hierarchy which is usually called protein complexes; the general definition is that already rather stable proteins of quaternary structure combine into large constructs capable of performing a multitude of actions, sometimes comprising entire manufacturing machineries. A classic example of these macromolecular complexes is the ribosome. 


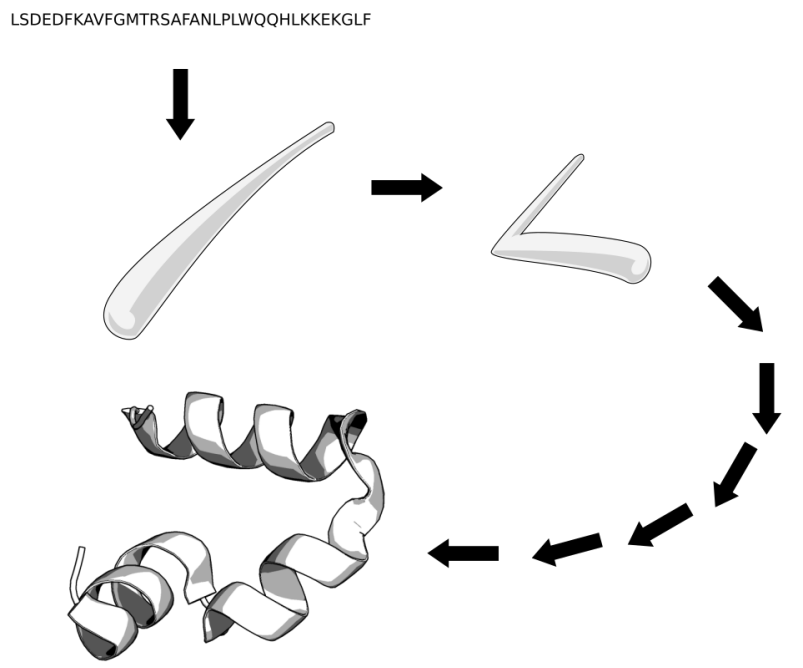

Figure 1.3: Illustrating the classic idea that a protein sequence folds through a series of steps into a compact native structure. In a more modern view, there is actually an ensemble of series within which the folding can follow many routes ending up into an folded state that is constantly transforming through the states of a whole ensemble of native structures. [49] The example shown here is that of the 35 residues long headpiece domain of Villin [32] available as PDB entry $1 \mathrm{WY} 3$ available at www.rscb.org [25]. The protein was rendered using the Open Source community version of the software PyMOL. [38]

One could go as far as to claim that there exists an even higher and more abstract notion of structural hierarchy; the pathway. Here, we are talking about a sequence, or network of mediated interactions and actions that are connected between proteins and their complexes via either direct interactions that are separated both temporal and spatial, or mediated by ligand proteins or other signaling molecules that carry the messages between complexes.

The focus in this hierarchy of this thesis is on the level of the tertiary structure, and how one can reason about the rather diffuse notion of protein domains. As will be discovered, there is an intrinsic connection to the folding pathway and that of the functional interpretation of the domain.

\section{Molecular evolution}

Whilst evolution in its broader sense involves the progressive acquiring and perfection of phenotypical traits by living organisms, molecular evolution focuses in particular on the molecular aspects supporting those speciation events. As molecular evolution involves all of the cells chemistry, such as DNA, RNA and proteins, this thesis has limited its interest in molecular evolution to proteins. 
It is of central interest to the evolutionary biochemist to distinguish relationships between the cells chemical components in order to understand their function with respect to the whole. Thus a primary interest is to understand what makes a constituent functionally homologous to another, in order to structure available information and enable parables from similar phenotypes to similar chemistry. On the protein level this chemical similarity stems primarily from the sequence, the primary structure of the protein.

Therefore, in this introductory section on molecular evolution, the discussion will be centered on polymorphisms of the protein molecular amino acid chain, a concept that is generally known as mutation.

\section{Mutations and Genetic Drift}

Amino acid mutations and genetic drift are two major factors that alter the functional protein expression within a population of organisms. The mutations are alterations of the expressed gene products from generation to generation which accumulate and give rise to genetic diversity. Diversification by mutations happens either by genetic damaging and error prone repair mechanisms [130] or by processes like those associated with pseudogenes and domain fusion and fission 64 138. Contrary to mutations is the action of genetic drift which tend to select dominant traits and risks eliminating rare features. [89] Genetic drift is also a factor in speciation, coming from its tendency to eliminate less abundant traits in rare individuals that in their similarity bridge two larger groups of individuals that are more dissimilar. Thus genetic drift can act as a selector of common traits while mutation provide the diversification.

Although these processes are fundamentally interesting in understanding evolution, the product of them can be readily seen as co-adaptive traces in current day databases that can be utilized by models with more modest assumptions. This thesis focus on such patterns of amino acid concurrency in residue positions observable in families of protein sequence homologs and use those patterns to infer functional relationships between the residues.

Having a notion of mutations defined, the next matter of concern is that of homology and how mutated protein sequences can be considered of the same kind and function, even when they are seemingly different in sequence.

\section{Homology}

Homology is the central concept that makes it possible for biologists to infer structure and relate different organisms traits and origins to each other. [118] As such, it is multi-faceted carrying different aspects, and can be applied to different levels of the biological structures.

On the level of molecular biology and evolution, the concept of homology gives rise to protein families and super families that encompass conserved sequences and structures. Structurally similar proteins are considered of the same super family even if their sequence do not imply that they are similar by sequence homology. [102] These superfamilies are then subdivided into smaller protein families which are centered on sequential similarity. [54 53] Families are an useful way of structuring protein knowledge since all members of both protein superfamilies and families are expected to have some sort of homogeneous function due to their apparent biochemical homology. It is such groupings of proteins into families that enable the attribution of function to conserved regions.

A fundamental assumption, used in the study of protein structures and sequences, is that evolutionary conserved regions are signifying parts that are functionally important. By conservation we mean the concept that a certain feature or trait is commonly recurring as an attribute of all entities within the set of entities under consideration. If then given a group of proteins with conserved phenotypical function and role in multiple organisms, and this group of proteins also 


\section{INTRODUCTION}

have part of their structure or sequence conserved, it is natural to conclude that this conserved structural or sequential region is also important to the function of that particular class of proteins. This importance is especially significant if much of the other sequence regions in general are not conserved throughout the protein family, as this pinpoints the conserved region as more or less the only common biochemical denominator of the family, thus suggesting an attribution for the function of the family to the conserved region.

In order to identify patterns of conservation in the allowable space of protein sequences for a particular protein family or superfamily, on need to identify the set of homologous proteins pertaining to the particular structure and function discussed. Finding homologous proteins in the sequence space is usually done by assuming that sequences pertaining to a the same superfamily, having the same structure or function, also has roughly the same or similar amino acids. In other words, the assumption is that the superfamily, to which the query protein belong, can be divided into subsets of protein families where at least one family contain the query.

It has been shown empirically that about $30 \%$ of sequence amino acid identity is required for sequence homologues to be roughly structural homologues as well, thus algorithms producing measures of sequence similarity can be used to identify homologous sequences. [20] The inverse of the homology by similarity criteria is not true however, since there are structural homologues that have a common fold but a sequence identity of less than $30 \%$. 96.81 .

Expectation is that more homology data are available for any given specific protein than can be discovered by using only traditional homology identification by sequence similarity. By collecting the set of sequences that maximize a similarity scoring, we can be reasonably sure that the top scoring matches are pertaining to proteins or protein fragments carrying the same structure or function as our query sequence. The set of proteins so found is however not the full set of proteins functionally related to the query, since it is expected that there are other proteins stemming from entirely different evolutionary origins that has evolved convergently into the same form and function, but having a rather different amino acid sequence. It is therefore intractable to find these convergently evolved by using the standard approach of sequence similarity alone. Thus we expect that there are more homology data available, in terms of protein sequences in the databases, than that we can find by mere sequence similarity alignments.

Also divergent evolving protein homologs can maintain the same function and structure while the sequence similarity is all but lost. As stated before, sequence similarity can arise from both divergent and convergent evolution, where closely related divergently evolved sequence homologs are readily detectable by standard approaches. Distantly diverged homologs can evolve way below the $30 \%$ similarity threshold, whilst still maintaining structural and functional homology to the query sequence, rendering these homologs hard to find. The sequence differences in such protein homologs often stem from divergent evolution where single mutations has occurred over time. These mutations can then be followed by compensatory mutations also arising over time, thus stabilizing the function and structure by a function known as epistasis. [52

The maintaining of protein function, while sequences diverge, is known as epistasis and will be discussed in the next section. Epistasis is central to the methodology discussed later that uses co-adaptation patterns discovered in databases to infer functional relations between protein residues.

\section{Epistasis}

"[...] epistasis, that is, the interaction between mutations through fitness [...]

- Figliuzzi et al. 52

In structure bioinformatics, the epistasis of a residue pair means their mutual ability to alter the spatial conformation of each other by mutation. [103] This definition of epistasis as the coupling 
of conformational perturbations to mutations connects epistasis on the molecular level through the coupling of local conformations to overall structure, function and thus phenotypical fitness. Therefore epistasis can be interpreted as the coupling of residues on the molecular level, to the maintaining of fitness on the level of the organism with respect to the selection processes of evolution. [52] This coupling between hierarchical levels, from organism to molecule, is therefore providing a direct link between observable traits and their underlying molecular mechanisms.

The force of selection on fitness combined with mutation and recombination processes is what drive the evolution of new protein mechanisms through epistasis; but epistasis provide the direction of evolution through the functional connections offered by protein promiscuity. [128] Proteins are in general functionally promiscuous which means that they can interact with more partners than required by their natural function. Given enough slack in evolutionary pressure, mutation can result in a protein that is even more promiscuous, enabling new interactions to start taking place within the host. [113] Such mutations can accumulate, if not pathogenic, and provides a mechanism for new systems to emerge. [127. 61] These new systems can then be actively selected for through fitness from corresponding new environments or selection events. Thus, since fitness on the molecular level is realized as epistasis between residues, it is epistasis that determine the functional promiscuity with respect to fitness; defining the pathways for evolving new functionality, in a cyclical relationship.

This model for evolving by protein promiscuity is one of two extremes, where the other model is the alternation between two states of relaxation and selection, depicted in Figure 3.4 of section 3.2. with the two forming end-points of a continuum of process characteristics within which evolution works. The relaxation-selection model described in the method section is more reminiscent of the classic evolutionary model proposed by Charles Darwin, with a focus on the survival of the population rather than on the individual. Another difference between the Darwinian concept of evolution and that of the relaxation-selection model resides in that the latter puts emphasis on the requirement of relaxation for mutational processes to take speed, posing the selection event as a mere focusing aspect of the process as a whole and not as a driving force ${ }^{2}$ On the contrary from this is the epistatic model, which emphasize the focusing agent being the driving force, realized as the detailed epistatic relationships. As such the combination of two models, epistatic and selection-relaxation, forms a continuum of processes that make up a broader definition of the evolutionary mechanism.

The two views of evolution is better juxtaposed by how rare and significant they define a selection event, which influence how they are manifested in genetic archives and fossils. In the epistatic model, the selection is towards small increments of mutations that only alter fitness and function slightly. Contrary to this, the relaxation-selection view emphasize that any mutation at all can happen, until the point at which selection happens and only the fit enough populations survive. Thus the difference is merely on the definition of the character of the selection event considered; how often is selection expected to occur, and how harsh will it be on the weaker individuals in the population. It is also this difference that makes the epistatic-like processes

${ }^{2}$ This proposed difference might stem from a common misconception, in which case it would mean that the author of this thesis also has designed some concepts on a slightly erroneous assumption. The Darwinian theory has often been interpreted as "The survival of the strongest", whilst it actually is written "The survival of the fittest". [37| This Nazi-like interpretation is contrary to modern authors, who are to this thesis contemporary, whose argumentation suggests that it is better interpreted as "The survival of the most varied". This is also bringing to mind that it was the rag-tag allied forces that won the war, not the stringent and (proposedly) perfected axis. However, the author of this thesis have not yet amassed energy and time enough to work through the full original works of Darwin, so he cannot surely discriminate whether this is a misconception of those that followed Darwin, or an error of Darwin himself. Should the misconception be on the side of this thesis author, which is highly likely, then the relaxationselection model can just be taken as another name for the Darwinian theory of evolution with the rest of the discussion taken intact as a modern extension to the classic theory. 


\section{INTRODUCTION}

manifest patterns in contemporary DNA and protein sequence databases while the selectionrelaxation processes is more evident in major extinction events visible in fossil records.

The theory of the relaxation-selection model implies that the differentiation process that creates diversity from evolution happens under states of relaxation; the more relaxed habitat the faster it goes. Since stringent selection means that, on the molecular level, epistasis will hold most mutations deleterious [113] which will tend to remove such variants by the well known process of natural selection. Such stringent natural selection that removes many new variants, before they get established in the population of variants, means that the variant generation will be slower with selection than with no selection. Without selection, in a theoretical state of full relaxation, all genes would mutate randomly and the most variation would arise with respect to time. Most variants that accumulate so over time would not be functional, since there is no guiding force to determine what is functional with respect to the habitat. Here the process of natural selection comes in as merely the focusing agent, selecting the variants that has accumulated beneficial co-mutations and improved or maintained fitness enough to survive and stay in population. Thus it would be expected that non-deleterious co-adaptive mutations would start to arise in later generations, after few initial generations of slightly less fit individuals, something that has been observed in mutational experiments. [113 Therefore it is expected that it is in the relaxed state of a population wherein the actual evolutionary recombination and mutation of the gene pool takes place, and the more relaxed the environment, the faster this goes ${ }^{3}$

Other mechanisms for increasing mutational rates include environmental factors that directly damage DNA, such as radiation damage [147] and oxidative chemical agents. Such DNA damaging events will activate the DNA-associated repair mechanisms and therefore heighten the incidence of error prone repairs. If working out a more precise model on evolutionary speed, all of these different aspects of mutation incidence modifiers needs to be taken into consideration. For the disposition here however, it suffices to mention these mutation modulators and instead focus on the general aspects of the evolutionary process as a whole.

Relating this interpretation of the evolutionary process to the current state of the human population leads to the tantalizing idea that the evolutionary pace of mankind is at the moment taking up a great deal of speed. This is contrary to a common belief that the evolution has more or less stopped for mankind, due to the lack of selection. Such a view stems from the assumption that in the lack of selection, when society takes due care ensuring the survival of individuals otherwise less likely to survive, that it would somehow be detrimental to the evolutionary objective for mankind on the large. ${ }^{4}$ Now, from the above discussion on the relaxation-selection model, we can see that this tending to our collective survival just means that we are in the relaxation epoch of the model, wherein we generate variation. Thus the current survival of most variants means that it is in fact thanks to this lack of selection that we actually evolve; it is variation that makes us fit.

Before moving on from this detour in misconceptions and their relation to the evolutionary theory described, it is better to clarify the authors assumptions on the extant misconceptions;

- Assumed misconception 1; Survival of the fittest $\Rightarrow$ Only the strongest survive

- Assumed misconception 2; No selection $\Rightarrow$ No evolution

${ }^{3}$ There is also another concept contrary to this that makes the variation generation process slow down. The more relaxed the environment, the less needed is each protein system. Since most cells have regulation mechanisms that will down regulate the translation of the gene products of systems not in use, this will also lessen the wear and tear on those genes. Thus the genes will less often get damaged, and they will therefore also mutate less often. So there is an equilibrium here, where we would expect optimal speed of evolution when the habitat is as much relaxed as possible, but still maintaining just enough activity so that variation generation takes place. By the way, do the reader see the connect to labor and market ethics?

${ }^{4}$ Again, some left over belief systems of the Nazi era. 
so, moving on.

With the epistatic model in mind, focusing on the digression of molecular evolution, the evolution process guided by the epistasis between residues should thus be evident as certain co-adaptation patterns within protein families that emerge over time. These patterns of coadaptation is expected to arise in homologous proteins as they represent a population of proteins owning the same function. Since the proteins of the same family own the same function they should also follow the same model of epistasis, and thus a pressure of fitness will keep the family's mutations within a certain pattern of allowable co-occurring amino acid types over certain residual positions. Therefore patterns in large protein families that are different to the expected background mutation would start to emerge over time.

Co-adaptation patterns of mutations in residual positions of a protein family can then be used to infer functional relationships between residues and be used to build more detailed models of the proteins of the family. Epistasis links the proteins residual mutations to that of fitness, requiring the mutations to stay in certain patterns compatible to the residues conformational relationships. These requirements reflect the molecular arrangement, thus the co-adaptive patterns that has risen out of epistasis also reflect conformational interactions between residues. Many conformational interactions are more or less static in the native fold of the protein, which means that there is a connection between spatial 3D nearness of residues and their epistatic couplings. Thus mutational co-adaptive patterns can be used to infer measures of functional connections and spatial distances.

Moving on to the concept of allostery, we will discuss the concept of functional relationships between residues on the molecular level, a concept which is closely related to that of epistasis. Since the coupling of residues, via fitness manifested by the forces of evolution on the organism level, is mirrored by the concept of allostery manifested by dynamics on the molecular level.

\section{Function and Allostery}

Allostery is the concept describing how distant parts of a protein structure is in functional connection with each other, a mechanism which allows proteins to form the basic link within signal pathways. An example is when the interactions with a smaller ligand or other protein, on one side of the protein in question, is changing the characteristics of the surface on another side of the protein, influencing the interaction with a third partner. Thus this transmitting of a proteins interactions from one partner to another partner forms one link in the chains of interactions that we call signal pathways.

The classical view of allostery, seen as a signal transmitted by the transition of a whole protein or domain between two conformational distinct states 92 75], need reinterpretation on a more detailed level emphasizing the allosteric pathway as contrary to conformational changes [112]. As new methodologies for studying the intrinsic mechanisms of protein domains has become available, a more clear picture of protein allostery has emerged. These details involve the interpretation of residues functionally connected throughout a protein structure as allosteric pathways. Such pathways are those that transmit the information about a ligand interaction in one part of the molecule to another distant part, not directly connected to the first part. This information is transmitted via changes in connected and immediate structural interactions traversing the structure residue by residue. Thus the allosteric pathway can be viewed as a path of functional interactions, being part of a larger residual network of interactions.

Such allosteric pathways require modern tools for analyzing the protein structure and their dynamics using a residue-residue interaction (RRI) network model. Residue interactions responsible for the allosteric signal transduction can only be distinguished from other nontransmitting interactions within the structure by their changed interaction patterns upon transduction. Therefore comparison of interaction patterns between different conformational states 


\section{INTRODUCTION}

of the protein can reveal which interactions that have changed and thus being responsible for transmitting the signal. Such interaction patterns can be readily discovered using a network model that emphasize the connectivity between residues.

As will be outlined below, not only allostery but function itself can be defined using the network interaction model. Therefore there exist a synonymous relationship (a morphism) between the concept of allostery and function; allostery can be interpreted as function and vice versa, the former being the mechanistic causation of the latter.

The thesis of this dissertation is centered on the idea that the protein structures and their connective interactions constitute a multi-layered network wherein the functional mechanisms of its subnetworks are defined through their connections to the network itself and the conditions of the native environment within which the living system is immersed. In more general terms this means that each function of each part of each protein within the network of the cell, which the proteins constitute, is determined by their interactions within the cell and, in turn, the cells interactions with its natural habitat. Such an idea builds upon the assumption that it is the holistic network that give rise to, and most accurately define what life is; interpreted as a holon [74] of self sustaining recurrent networks, self similar on all scales of temporal and spatial dimensions.

Thus, in their highest abstraction, proteins are logical blocks or nodes in the networks that constitute living organisms and the proteins themselves are also expected to be constituted out of networks of smaller components of logic. Ranging from classic domain definitions [96], to smaller entities such as protein sectors [112] and foldons [49], to amino acids; all of which are being reused in a myriad of different combinations, thus generating the plethora of proteins and their functions found in nature.

A condensed description of these logics are best centered on their syntax, which means the way they interact with each others; to this end, we find that the best descriptor, closest to this real syntax, would be the physical interactions manifested by proximity contacts between the residue nodes (amino acids) constituting the protein domains and complexes.

Modeling residual interactions is of interest since it puts into relation the actual structure of a protein to its role in the network of proteins within the cell, i.e. the protein function. Protein function is usually defined in terms of gene ontologies [17], or GO terms, which make out a controlled vocabulary for describing function and other features of genes and their associated products.

As pointed out by the GO consortia, protein function is a problematic term due to its ambiguous nature. [17 114 The term might refer to either or all of biochemical interactions, scaffolding, cell structure and biological roles which all appear at different scales of the biological system considered. It is hard to pinpoint the characteristics responsible for all of these functions at the protein level, since biochemical reactions happen at the atomistic nano level, scaffolding on the polymer scale, cell structure on the microscopic scale and so forth.

In order for proper (i.e. automatic) attribution of function from known proteins to newly discovered entities, we are in need of a functional definition that is better equipped to scale between these levels and more correctly attribute the different aspects up and down the hierarchy. Promising are models that use gene and protein network interactions of whole organisms to compare their interaction patterns and attribute function cross species. [141 114 These methods are modeling proteins as entities in a network of protein-protein interactions (PPI), which is a model similar in form to how a protein structure can be expressed as a network of interacting residues. Therefore, these network representations are promising, since the structural similarity between RRI and PPI networks provide a common framework for the two views. Through this similarity, it would then be more straightforward to attribute certain functional propensity to a group of residue interaction patterns, down the hierarchy, from that of protein entities. Such 


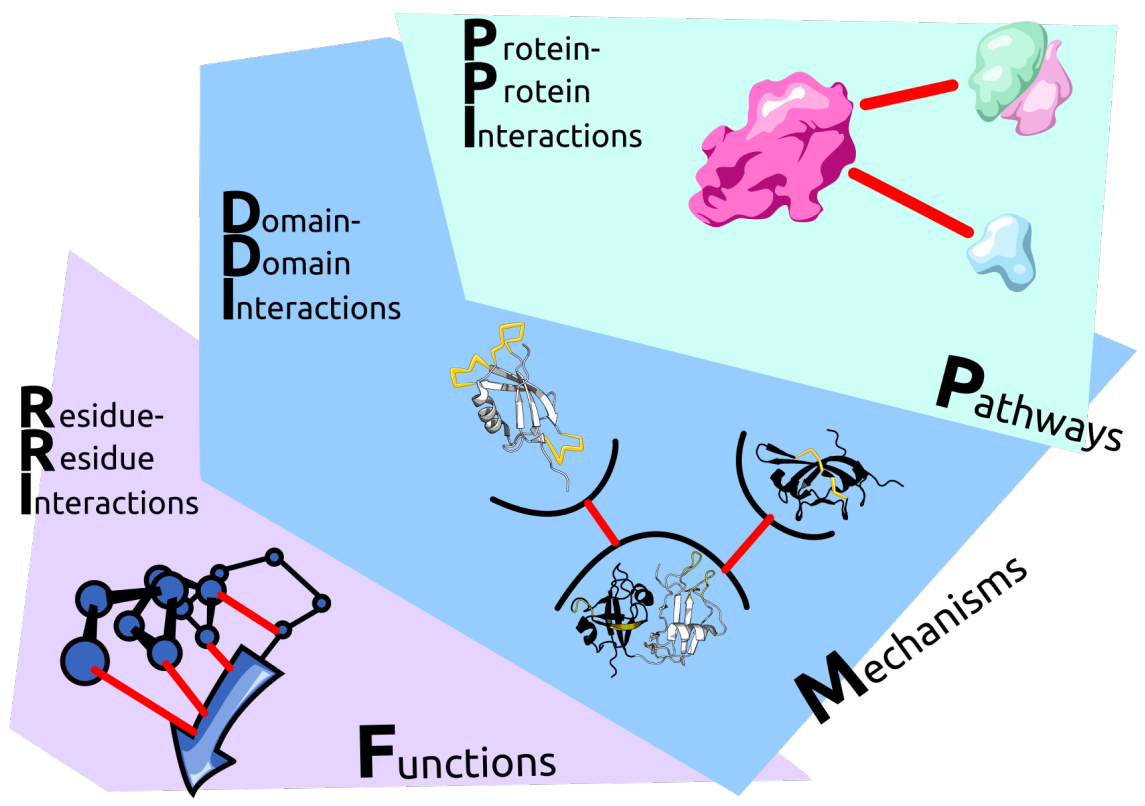

Figure 1.4: Illustrating the RRI through DDI to PPI conceptual hierarchy. Indicates that network interaction walks can be interpreted as functions, mechanisms and pathways on the corresponding levels.

hierarchical modeling could serve in identification and understanding of the underlying mechanisms in the related groups of GO-terms.

Restating the above differently, models similar to those resulting from protein RRI network predictions are also being used in propagating functional annotations (GO terms etc.) over predicted PPI networks. On the PPI level the interaction network is modeled as a graph resulting from various sources of PPI predictions [94] where function is predictively propagated over the graph. In comparison, the epistatic models [70, 47] illustrate how RRI networks are predicted from co-adaptive data detected in protein families. Similarities in such RRI networks between proteins could then be used to infer functional similarity between proteins, which in turn can aid in edge completion inference in PPI networks. Further functional couplings on the PPI level can be extracted from RRI networks based on the evolutionary tendency of modular reuse of RRI functionals. It is therefore tempting to consider using RRI predictions as raw material for PPI predictions, then propagating functional annotations across the PPI network and in turn down to the associated RRI networks, revealing the underlying mechanisms.

Further strengthening the network paradigm are results from studies that has been using the network model to attribute similar function with sequence homology as measure of nearness, suggesting that the network model for protein function and structure is universally sane. Basically all three instantiations of the network model is making the same assumption. First is the PPI network that assumes that proteins that interact with the same kinds of proteins are probably having the same function. Secondly, the sequence homology network wherein proteins that have the same function also score high in homology to the same proteins. And thirdly, in the RRI network where residues that are connected to each other and have a similar neighborhood also 


\section{INTRODUCTION}

carry a strong functional connection. All these similarities in the interpretation of the network model on the different levels carry the same assumption; if two nodes in the network has the same neighborhood, then the two nodes are also functionally related. Such similarity in both the interpretation and the structure of the network model strongly suggests that the model is suitable as a starting point for designing a multileveled hierarchical model as depicted in Figure 1.4

For an accurate, effective and plastic hierarchical arrangement to be realized there must be a common model, enabling an interpretation of the function concept on the residual level. It is in making this connection from protein function on the protein level, to that of the interactions on the residual level, where we find interest in the protein domain modularity and domain-domain interaction (DDI) network concepts that are discussed next.

\section{Modularity and Protein primes}

A common concept in protein modularity is the protein domain, defined as the subunit(s) of a protein sequence that can fold into perceptually distinguishable shapes, independently of the rest of the protein. Mainstream approaches to understand protein domain structures and catalog them focus on a description of homology, within and between protein families, by constructing a hierarchy extending upon the classic phylogeny view of genetic heredity. [96 101] Here the protein folds and homologous sequences are organized in a tree-like hierarchy which branches out from common tertiary structures of superfamilies into smaller families with more stringent demand on sequential homology. 102. 53 This view is important in understanding protein relationships and transfer known function between similar entities, but it does not help in deciphering the underlying molecular mechanisms as the protein domains themselves are treated as more or less holistic units.

Contrary to the more holistic phylogenetic models, similarities between smaller modular elements has been observed between domains of different ancestry, giving reason to look into the structural hierarchy and not only the ancestral one. [8] Smaller elements can always be partitioned out of a whole, but if it is done without any underlying assumptions one risks ending up with bits and pieces which cannot be attributed any real meaning. An example is the partitioning of a protein sequence into its amino acids; we know the chemical aspects of most amino acids but the procedure does not give us much insight into the function of the protein by studying the amino acids separately. If one instead divide the protein into smaller entities that also carry distinct mechanisms that relate to the function of the whole, then these parts will start to relate the domains function down to its mechanistic background.

Whilst homologous elements of larger domains has been identified, epistatic models of protein domains has revealed that domains consists of smaller distinct functional protein sectors that communicate by allostery throughout the protein structure, providing a basis of functional meaning. 62 Protein sectors form smaller and dense sub-domains that are evolutionary coupled via co-adaptation patterns detectable in protein families, patterns which suggests that sectors arise from epistatic networks. As the sectors also form distinct functional networks with respect to allostery, they seem centered on the mechanistic elements underlying protein function. Since these webs of interactive protein sectors are expected to be common to proteins in general, such protein sectors could constitute a functional basis for modular evolution and a meaningful partition of protein domains into smaller entities.

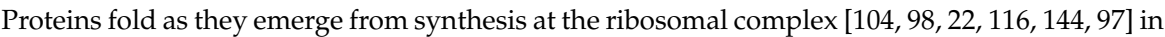
a hierarchical manner as depicted in Figure 1.5 The ribosome is a large enzyme complex of multiple protein chains and enzymatic ribonucleic acid (RNA) that is largely common in form and sequence to all cellular lifeforms and responsible for virtually all protein sequence synthesi ${ }^{5}$

\footnotetext{
${ }^{5}$ Mind there are man made synthetic amino acid peptides.
} 


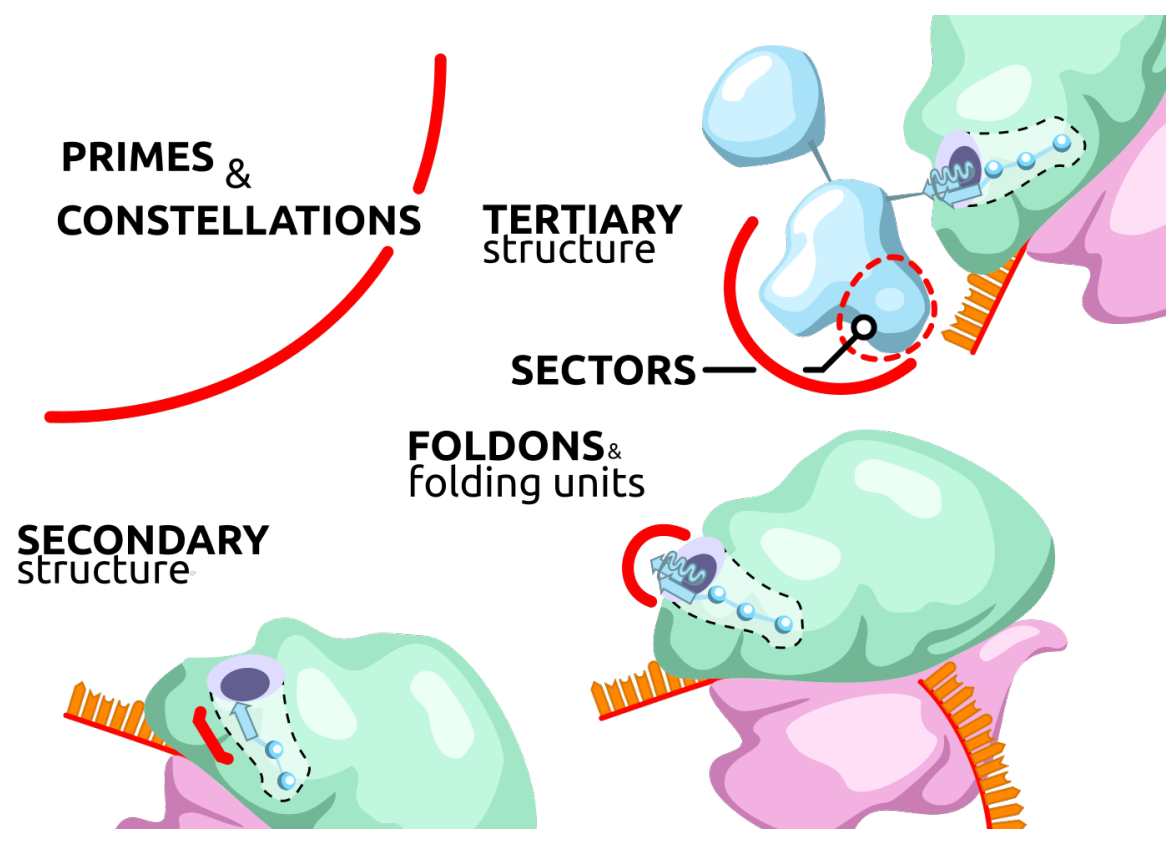

Figure 1.5: A simplified illustration of the sequence when a protein folds on the ribosome. The protein domain architecture concepts of folding units [80], foldons [49], sectors [62], primes and constellations are indicated by red in the hierarchy. Primes and constellations provide a model for all levels of the hierarchy as they focus on the interaction network, supporting a fully modular view of protein structure centered on a proteins functional aspects.

This large complex is what translates the genetic code from messenger RNA, which has been transcribed from deoxyribonucleic acid (DNA), into the protein peptide sequences that fold into functional proteins. Pioneers in bioinformatics proposed that proteins fold by first forming folding units after the initial secondary structure has formed. [80] Since the inception of the high resolution ribosomal structure model [144], it has been suggested that this process happen partially inside the ribosomal funnel [59]. This nascent domain view has found its critique largely based on the assumption that the ribosomal funnel is too narrow to encompass a fully folded domain, even if the domain considered is one of the smallest known. [143] Such critique could be largely unwarranted since the domain considered is larger than what one normally would consider as a folding unit [125, 80]. The nascent folding process, partially inside the ribosomal funnel, has also been observed by more recent studies. |76| This all comes together as what is now known as the foldon theory [49], which suggests that protein domains folds by forming an initial smaller folding unit called foldon that acts as a nucleus for subsequent step-wise foldon formations. These foldons can be seen as the units which are the fold mechanical counterpart of what we previously have described as protein sectors from the view of epistasis.

Now that we have a description of smaller sub-domain entities from the view of epistasis on protein sectors [62] and another from the fold mechanical view of foldons [49], we need the concept of allostery to connect these entities by their homology as functions. Moving away from the definition of homology as sequence similarity (protein sectors) and the folding process (foldons), 


\section{INTRODUCTION}

one can instead center the concept of homology on the network model of function. Such a view yields links of homology from the point of view as a syntax of interactions; a network that has the same kind of interactions as another network is essentially a homologous network. It makes clear sense to use network homology to infer sameness between protein sub-domain entities, since it is in these networks that we also can identify function interpreted as allostery.

With this allosterical concept of protein function expressed in residue connectivity, the identification of logical networks or blocks centered on function is made concrete by adhering to the reasonable definition that arise from the two rather distinguishing features enumerated below;

1. a logical block, or protein prime of a simple function, form a densely interconnected network as defined by allostery and epistasis, that at the same time

2. show a well defined interface with some, but not all, of other such primes, yielding a sparse network of connectivity between primes, each carrying a simple function.

In this way protein primes becomes a network based realization of sub-domain entities that is centered directly on function and not on form nor sequentiality (Figure 1.6). As protein primes are defined purely as networks, they can reflect multiple sectors from multiple families, should they somehow be governed by same epistatic network and have identical allosteric pathways. Furthermore, protein primes can also reflect the connectivity within multiple distinct folding units, as different sequences can give rise to the overall same topology and connectivity of a folded subunit.

Thus, a collection of protein sectors and foldons that do not stem from the same protein family, nor have the same sequence of secondary structure, can still pertain to the same function and thus the same protein prime. As protein primes are defined as the connective network and not the form, they describe what is understood as function. Since there are multiple protein families and folds that can form partially the same RRI networks and provide similar channels of allostery, these can also be modeled with similar network models, meaning that protein primes render a measure of homologous function (Figure $1.6 \mathrm{c}$ ).

As mentioned, a third homology aspect centered on functionality, from which to view and catalog protein sub-domain entities, arise from the concept of protein primes. This view provides a way to relate different sectors and foldons via primes as a measure of homology. Primes also provide a homology that can be used for building hierarchies based on functional relationships. These functional relationships between primes can be expressed as the complex functions of prime constellations and the simple functions of isolated protein primes.

Prime constellations are loosely defined as the set of primes that make up a protein or domain (Figure 1.6 a). They can feature different number of primes and do not necessarily have to encompass a whole domain or protein; the only requirement is that a constellation can be divided into a set of clearly defined primes of simple function. The concept of simple function is distinguished from that of a complex function on the basis of modularity and is another way of inferring protein prime constellations.

The parity of the concept of a more complex and another more simple functions stems from the notion of functional, or allosterical connectivity, posing a secondary way of defining the concept of protein prime constellations. A more simple function is one that is built up from a more discretized logical block than that of a complex one, such that there is a clearly distinguishable sub-network that encompass the simple function centered on a prime. This prime should be clearly rendered within the network with respect to the rest of the protein in consideration. Such an abstract block of an simple function is distinguished from other more complex blocks of functions by featuring a more even distribution of its internal node connectivity than in the distribution of connectivity to other blocks. A complex function is on the contrary made up 
a

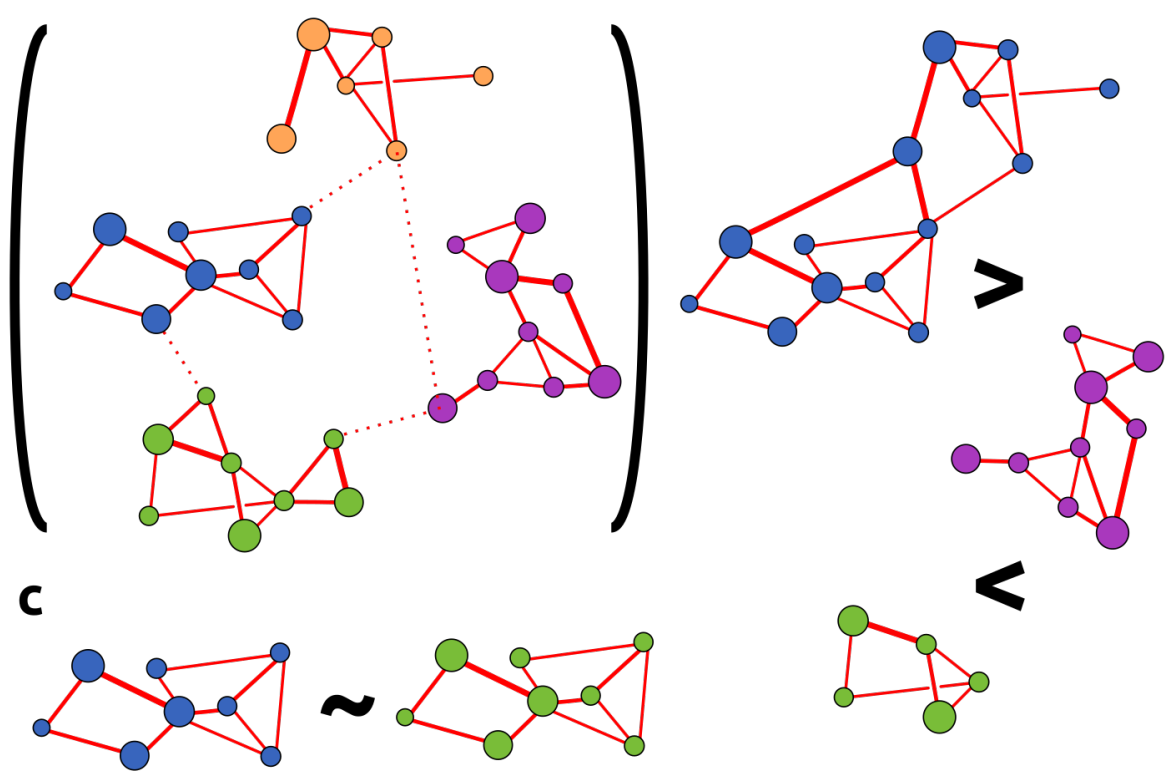

Figure 1.6: Protein primes are best realized as clusters of nodes in network models. They are here depicted as nodes with one color per prime, and their interactions indicated by edges. Distances does not signify real distance, as it is just a sorting; it is the connections that signify functional relationships. (a) There are prime constellations consisting of primes carrying simple functions that are not constellations themselves. They form clique-like structures in the constellation with weaker interactive patterns between them than within. The more primes in the constellation, the more complex it is. (b) Some functions are expected to be larger than other primes, here three are depicted from the bottom up in growing order. Just as with prime numbers, it is expected that there could be functions that are increasingly large but cannot be split up in smaller well defined primes. The minimum size of primes needs to be determined from a view of utility, which will have to be evaluated with respect to some assumptions (i.e. axioms). (c) Protein primes are characterized by their internal and external interactions only. Here, two analogous primes are illustrated, though without any indication of external relations. 


\section{INTRODUCTION}

of a heterogeneous mixture of multiple interleaved blocks of function and must therefore be a constellation of protein primes.

Protein primes should also perform a simpler mechanistic function than prime constellations since the allosteric network that constitute the prime is more well defined than that of the constellation. The prime consist more of a "chunk" of material as contrasted to a complex function or constellation which has a more delicate structure with interleaved densities of interactions on different levels. Within such denser material the number of modes of actions are also expected to be less than in the more complex one, due to the smaller number of degrees of freedom. Thus this yields us the notion of a complex contra a simple function expressed as, and quantified by, network connectivity. Next, the notion of small and large protein primes is explored.

There are to be expected increasingly large primes constituted out of larger interconnected networks of residues that cannot be said to be prime constellations as they cannot be partitioned into smaller and simpler well defined primes (Figure $1.6 \mathrm{~b}$ ). As a morphism to the natural number system we have a concept here much like that of prime numbers, where increasingly large networks can arise that do not constitute a constellation of smaller prime functions but define an entirely new and unprecedentedly large prime function. Thus by this definition of small and large, one can expect to find both smaller and larger simple functions manifested as the potentiality of discovering increasingly large residue node networks that cannot be partitioned into previously cataloged smaller primes.

This model of primes and constellations do however break down in particular situations; one example being due to functional emergence and another example is the structural collapse. An emergent function is one that is built by multiple primes, thus being a constellation, but in itself having a function alien to the set of primes that constitute it. In this way, an emergent function of a constellation cannot be described as the functional summation of the primes as perceived in isolation. Since such an emergent function could arise while still maintaining the prime structures the model breaks down. This breakdown by functional emergence is perpendicular in nature to the model breakdown stemming from prime structural collapse.

The other mode of model breakdown can be described as the concept of prime structural collapse. If the interactions between the primes energetically favors an alteration of the internal prime interactions it can promote a collapse of the prime internal structures. Such collapse of the prime structures will result in the formation of new primes; thus in effect altering the prime definitions in the constellation, while the sequence is the same. This is best viewed as being part of the folding process and therefore a problem of protein design, where the correct prediction from sequence to interactions and function is paramount. In such predictions you would probably be able to predict some prime constellation using prior knowledge, but as these primes interacts there is always the possibility that they might combine into something that is more energetically favorable that has yet to be documented. As such, any prime constellation predictions performed on such a de-novo sequence must be considered as part of an iterative process, where the folding mechanics are taken into consideration, thus expanding the model into temporal space. Otherwise one risks that, given a constellation designed and synthesized using a set of poorly predicted primes, these primes might in reality perturb into another set of primes due to prime plasticity, and result in a functionality not described by the primes used to construct the constellation in the first place.

For protein design, one would therefore need to take into account any possible prime plasticity, and would have to understand a temporal relation between primes which expand the model complexity and relates it to the concept of foldons. One need to model what happens in the folding process on the ribosome, i.e. given the sequence of synthesis and folding (Figure 1.5; what primes will arise at first and how will these merge and combine into new primes, eventually forming a stable constellation? This modeling is in large outlined and described by the foldon theory mentioned above. [49] By understanding this temporal relation, marrying the 
foldon and prime perspectives, one could potentially start to reverse engineer the folding of a set of desired primes and thereby design a protein from function to structure to sequence.

In the setting of this thesis, being presented with already folded protein conformations, neither of these routes of model breakdown poses any problem. As one is presented with the final conformation of the peptide considered, the problem simply becomes to understand what primes are already there. Thus, one do not have to consider how the primes formed initially, reducing the problem of prime plasticity and emergent functionality to the simpler act of identifying a new set of primes.

Thus we have discussed three theories on protein domain hierarchy structure; the folding units and foldons [80, 49], the statistical allostery centered protein sectors [112] and the interaction centered prime theory presented here. It is important to emphasize the differences between the three theories and in particular their central views. Folding units and foldons discuss the folding pathway and the mechanics involved in the folding process and centers on the sequential folding of the protein structure while the protein sectors depict the interaction network of a particular protein once it has folded. These two views is contrasted against the more general protein prime setting, as the prime setting only considers a particular network of interactions and do not discriminate between particular proteins who carry the same prime functions, even if they have different sequences and/or folds. As such the protein prime model reflect the general function in its bare essentials, whilst the foldon and sector views put emphasis on the particular protein. It should be noted that even though the classical definitions of the protein domains closely relate to the idea of primes on some level, such as the definition of the SCOP common fold [96], these models do not discuss the hierarchical aspects of domain formation as does the prime and foldon theories.

As protein dynamics is discussed next, the concept of protein foldons and primes graciously connects to the dynamical nature of the intrinsically disordered protein discussed thereafter.

\section{Dynamics and Function}

In this section protein dynamics are described as a mechanism governing allostery, offering on one hand support for the interaction view of function but also explains the idea of dynamics as function.

When protein dynamics are considered, a modification is prompted in the dogmatic view of Anfinsen on protein structure.[12] Proteins seems not to assume just a single state, but instead perpetuate to traverse an ensemble of conformations over an energy landscape. [56] This modifies the energy well in the original thermodynamic hypothesis into a energy valley, wherein the protein molecule transform continuously through multiple similar but distinct states.

Through the realization as the interconversion between multiple conformational states, protein dynamics provide a mechanical basis for allostery and signal transmission within DDI and PPI networks. [29] For allosterical signals to be transmitted between protein domains and through interaction pathways, they need to have a vector of transmission. This vector is realized as the dynamical movement of residues within the protein structures, that transform the conformations continuously from one state to another. Such transformations can create concerted motions of residues linked in chains, forming pathways of allosterical connections throughout the structures, connecting distal sites. [112] These joint motions of protein residues and domains are therefore what outlines the underlying mechanical foundation for protein allostery and functional signal transduction.

Furthermore, whole domains are expected to be moving with relation to each other, changing the interaction patterns over time and providing functional mechanisms. [65] By pivoting on hinges and rotating around connective axes, domains can change the overall structure of the protein they constitute without altering their internal structure. In this way mechanistic 


\section{INTRODUCTION}

functions can arise from the mechanical perturbation of the proteins interfaces or that of any connected protein partners.

As not all conformations are visible in the models provided from experiments, dynamic simulations are warranted to explore the full functional interactions available to protein systems. [55] Multiple conformations can be discovered for protein systems but, depending on the technique used, these conformations can be limited in number and might not express the full interactive variability of the system. Models are available that can interpolate between known conformations, revealing a more complete but still limited picture. [77, 4] Therefore, to unlock even more information on the interactive functionality of the protein systems, protein dynamics simulations are warranted.

Some protein dynamics are expected to be functional providing successions of interactions, or lack of interactions, that carry functional implications for the system under consideration. [131] Parts of the protein system might remain unstable while other parts are forming stable interactions, providing character to the function that involve the lack of interactions. Further dynamical aspects of function can be realized as interaction patterns that develop over time.

Structure centered views of protein function has problems capturing such dynamic functions, whilst both succession of interaction patterns and the lack of interactions can be modeled in a network model such as the one describing protein primes. Interaction centered models that work from the structural depiction of protein interactions make the assumption that it is the static interactions that are of importance for function. [6. 5] These views does not support the possibility of functionality within the patterns of succession of interactions in addition to the mere interaction patterns. The protein prime and similar models based upon networks of interactions must not make the same assumption. Network models can encompass a succession of interaction patterns by expanding the patterns into temporal space. Such expansions are readily realized as formulating the interaction patterns in the network models as tensors rather than ordinary matrices. Therefore protein primes and other network models are much more suited to approach dynamical function than the structural view.

Another impact that dynamics has is on the evolutionary aspects since, from the epistatic view, dynamics provide a mechanism for protein promiscuity. [129] As protein dynamics are not expected to be perfect, pertaining to the proteins current main function in the host, only a subset of the proteins conformations is expected to be related to this main function. There might however be other conformations that carry less functional roles in the proteins native setting, and those conformations could possibly support other interactions with novel function. Should such other interaction possibilities arise and become selected for, then it would instigate an epistatic pressure over which the protein could evolve into this new function via the dynamic ensemble. Thus dynamics provide a plausible underlying mechanism for epistatic evolution by protein promiscuity.

In the next section we go into detail about the intrinsically disordered proteins, proteins which poses central in parts of the discussion on the dynamical functions highlighted above.

\section{Intrinsically Disordered Proteins}

In the previous section, Anfinsen's old dogma [12] needed an expansion in complexity to explain protein dynamics. Now, the concept of intrinsically disordered proteins (IDP) that is described below challenges the central dogma of genetics instead, as these IDPs takes ensemble structures to an extreme when some IDPs seem to retain function while owning no structure.

Contemporary understanding is, contradictory to the sequence-to-structure-to-function view, that not all proteins has to fold into stable globular structures to acquire function. Indeed, it has

*and Function 

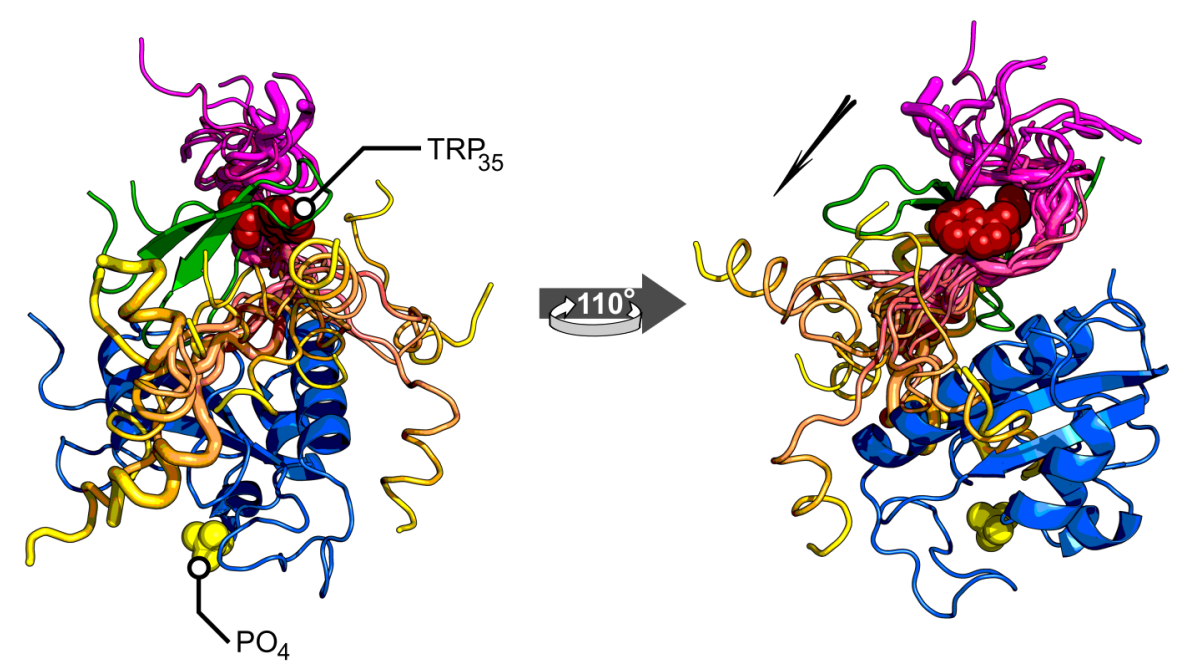

Figure 1.7: A bound sub-ensemble model of the c-MYC IDPs [134] MoRFslike[136] MB0 in fuzzy complex [131] with PIN1[66]. Tryptophan 35 in PIN1 is indicated with red spheres, PIN1 bound $\mathrm{PO}_{4}$ with yellow spheres and the black arrow indicates the $\mathrm{N}$ - to $\mathrm{C}$-terminal direction of the sequence of c-MYC $\mathrm{MBO}_{\mathrm{O}}$

been established that there are proteins that lack stable structures and still maintain essential functions pertaining to their systems. Such a class of unstructured functional proteins are the IDPs, which provide function without necessarily forming stable interactions with their partners. [41] IDPs function is mediated either through folding upon interaction or by maintaining dynamic ensembles that influence the partner molecule in a fuzzy complex (Figure 1.7). 131] This fuzziness of IDPs interactions provide another route for protein allostery and signal transmission, thus adding another dimension to the space of protein functionals. [58] It should here be emphasized that this additional descriptor of protein function is not necessarily centered on structural interactions, but rather on a more dynamical aspect of molecular interplay. These additional routes of information transmission seem essential for higher organisms whose survival strategies rely on multiple regulatory processes that involve IDPs. [45, 135, 115]

The intrinsically disordered (ID) class of proteins are more prevalent in higher organisms 42 . 43] and figure predominantly in regulatory processes and pathways. Higher organisms such as eukaryotes and multicellular organisms seem to use regulation as a means to attain more functional variability. [115] Such requirements of more variability in higher organisms could stem from these variations functional involvement in processes that enable communication between cells in different tissues, facilitating differentiation. $|135|^{6}$ These regulatory processes are intrinsically connected to the IDPs which support a wide number of modulating events involving post-translational modifications, which are often featured in conjunction with many-to-one and one-to-many molecular interaction hubs that resides in signaling and processing pathways. 34. 63. 99]

\footnotetext{
${ }^{6}$ There is a simile from this need of greater regulation in multicellular organisms to the need of greater intricacy in legislations for an urban environment of human populations as compared to those individuals living by themselves in rural settings.
} 


\section{INTRODUCTION}

Post-translational modifications (PTMs) are providing a means of modifying biological molecules further after their translation and synthesis, adding even greater levels of complexity to protein interactions and enable more diversified functions. The most prevalent of the known PTMs is phosphorylation [73], which is the covalent ligation of phosphoryl groups on amino acid side groups in the protein peptide chain. These processes of phosphorylation are facilitated by protein catalysts, or enzymes, known collectively as kinases. In part, it is these phosphorylations by kinases that are involved in enabling the cells to respond efficiently to new and large changes in the environment, without having to resort directly to mutation. 133 Thus such PTMs provide a mechanism that expands the adaptive functionality of the higher eukaryotes and multicellular organisms.

As such, PTMs an the dynamical aspects of IDPs adds more layers of complexities in the cellular arsenal of function, which presents a more mixed underlying mechanism for function than mere interactions. [43, 79] PTMs provide a form of allosteric regulation for IDPs that cannot directly be reflected as interactions between the functionally affected partners. Additionally, by themselves, IDPs dynamical and fuzzy aspects provide more pathways of allostery than mere interactions. This expansion of function from notions separate in nature from structural interactions suggest that the standard structure-function view only include a subset of the total functional space of proteins in general. Therefore, any model that is able to encompass the full functional space of proteins cannot be restricted to a viewpoint wherein function is described as mere structural interactions.

In light of these complexities, a network model such as the protein primes model could be used to facilitate a functional description of IDPs. Since a network model just depicts the relations between the contained network nodes, it is agnostic of spatial nor temporal locality. Relations can be expanded in temporal or other dimensions by simply adding more layers in the models. These layers can then be collapsed providing statistical images of the system over time or whatever particular descriptor it was expanded over.

As an example, such layers can be from multiple snapshots of interaction networks of a fuzzy complex evolving over time in a simulation. This multi-layered network can then be collapsed back to a single layered simple network again to provide a statistical picture of the system. The resulting model then convey the functional coupling within the fuzzy complex as measured by spatial proximity over time.

A measure of function used in the network model can thus be anything that has been shown to be suitably reminiscent to organism or system fitness. The aim of modeling is to understand what functional relationships that are at work, contributing to the proper function and survival of the organism. Therefore its natural to use a measure that is descriptive on the molecular level yet still reflect organism fitness.

Such measures of organism fitness on the molecular level is readily realized as epistasis [52], which can be employed to identify functional connections within IDPs. Fitness measured as epistasis can be detected in co-adaptive patterns of homologous proteins. As homologs are readily found in protein families, methods for family extraction and epistasis analysis can be combined to infer functional couplings. [16. 47| In this thesis, and in other works, epistasis has been employed to study function in IDPs. [132, 108]

Furthermore, since also IDPs seem to contain small functional modular elements, it is a tantalizing idea that they also could be supported by the protein prime model. Evidence exists that IDPs contain functional short molecular recognition features (MoRFs) [136] and transiently structured islets that form fuzzy complexes upon interaction [10, 134 66]. These phenomenons are similar by two analogies to that of the foldons of the foldon theory [49]. First, MoRFs and their fuzzier cousins are smaller and relatively condensed parts of their constellations. Secondly, as foldons fold upon interaction so does MoRFs and, to some extent, also the partially fuzzy islets. As 
foldons previously (Section 1.3) was described as an incarnation of protein primes, so can then these shorter ID regions (IDRs) also be thought of as primes.

In some aspects, due to their analogy, IDRs can be thought of as immature or highly evolving foldons. For the construction of this analogy, three similarities are drawn to that of foldons and globular proteins. First, some IDRs fold upon recognition just like foldons, providing the simile to the foldon units. [136 49] Secondly, the more flexible loops between the MoRFs, or other more functionally conserved regions [115], can be regarded as longer counterparts to the flexible loops of a folded domain. And last, the high evolutionary speed of some IDPs [27, 82] indicate that they might be under active selection for a new immature function via a route of epistatic evolution through protein promiscuity. [40, 129. 128]

The last corollary above merits a more detailed disposition in motivating IDRs as evolving foldons. The corollary stems from that IDPs has highly variable ensembles of conformations. These ensembles provide a broader array of adaptive interaction possibilities that has a low energetic barrier in comparison to the IDPs native functions. [40] Such adaptiveness provides the protein with functional promiscuity which can be realized as a less steep gradient for evolving new functions. [129] Thus, in this view, IDPs can act as an extreme form of a vector for epistatic evolution through protein promiscuity. [128] Furthermore, the very origin of the IDPs might stem from processes that recombine genetic material en masse, as such operations are likely to result in genes of initial low complexity. [64. 138] Taken together, this can be described as IDPs being vectors for the evolutionary development of new protein primes merging into novel constellations. However, not all IDRs can be considered to be evolving collections of foldons since some aspects of IDPs has direct functional implications which can be considered relatively mature with respect to evolution. [131] The existence of such mature IDP native functions are also supported by the incidence of relatively slowly evolving IDPs. [27. 82] Therefore it is plausible that IDP acts both as a vector for fast and adaptable evolution, while also lending more functional variation to the cellular machinery.

To summarize, IDPs puts another nail in the coffin of the central dogma of genetics, providing pathways of allostery and function not realizable as structural interactions and indicates a putative mechanism for protein prime evolution. Next is a short description of the molecular systems studied in the published articles included in the thesis.

\subsection{Systems}

In the published articles of this thesis there figures two molecular systems; one surrounding the human c-MYC and Pin1 protein interaction, and the other involving Pseudomonas aeruginosa's MexR protein and its DNA interaction.

The reader should already be slightly familiar with the nature of the first system c-MYC, an intrinsically disordered protein involved in the cell differentiation and proliferation. [134] cMYC is phosphorylated by its kinase interactor PIN1. In this thesis, the c-MYC-PIN1 interaction is investigated for its role as a molecular timer. [84] [83] This timer is of particular interest due to c-MYC being an intrinsically disordered phophoprotein implicated in cancer pathology. [91] The full c-MYC N-terminal IDP domain is also investigated for conserved islets and their epistatic relationships are cataloged.

MexR is a DNA binding protein responsible for the regulation of a multi-drug efflux pump. [142, 107] A particular mutation (R21W) [69, 3, 11] of this system was investigated by molecular dynamics to hunt down the mechanical background for its pathology in multi-drug resistance in the deadly bacterium Pseudomonas aeruginosa. 



\section{Thesis}

The thesis of this dissertation is centered on the idea that the protein structures and their connective interaction network constitute one aspect of a self defining syndiffeonic syntax of logic [78], which comes into being purely out of the conditional interactions within the network immersed in its native environment, i.e. the protein network within cells in their natural habitat.

It has previously been outlined how this theoretical network relates to protein residue interactions and their clusters, which has been a central point of investigation in the parts relating to this thesis of the principal articles included. However, as the works of this thesis is parts of other larger endeavors, in order to point out the essentials being part of this thesis, a descriptive summary of the aims follow below.

(Divisibility) Larger proteins should constitute a constellation of primes, each carrying a simple function. Therefore we should be able to observe smaller fragments in a constellation (protein), not necessarily sequentially connected, which by themselves constitute a simple function as an interface to one or a few other smaller fragments from within the same or other constellations. The investigation into c-MYC and its boxes explores this potentiality.

(Modularity) A protein prime of a single simple function is observed in multiple constellations of different function. After identifying primes within one constellation, we should be able to find these across other constellations in the known databases such as UniprotKB (sequences) and the PDB (structural models). Upon searching for analogues of the protein primes identified in an originating constellation, for an example the c-MYC protein, any finding of other constellations containing both primes of the originating constellations and other primes not present in the origin will serve to differentiate the function of these other constellations from that of the originating constellation, thus corroborating the corollary.

(Function) The function of a protein prime is defined by its pattern of interactions with other primes in the prime network. Given a constellation and defining its prime structure, a perturbation of the interactions between the primes should be enough to alter the simpler functions of 


\section{THESIS}

the primes involved in the constellation, and thus also the complex function of the constellation as a whole. Investigation of a seemingly benign mutation R21W in the MexR constellation reveals information about how the residue node network alters its character due to this modification; i.e. the interaction patterns within the residue node network of the protein constellation is severely affected by this alteration.

(Connectivity) Protein primes are internally more uniform and dense in their connectivity than the primes are in their interaction interfaces with other primes. This should reflect in a higher energetic stability of protein primes in general. As such, they should be observed intact more often than not in low energy decoys generated by protein structure prediction softwares that optimize any kind of pseudo-energy function, given they in some way resemble the true energetic relationships governing the native structure of the protein. In application of this idea to CASP, it translates to the possibility of finding protein primes prevalently folded in the top predictions of modeling softwares in the model quality assessment genre of the experiment. This prevalence is due to that protein primes should be centered on protein function, functions that in turn should be related to how native structures naturally are constructed and therefore also be reflected in the energetic models of the softwares generating the structural decoys. A corollary that stems from the basic fact that most such softwares are trained on known native structures. Thus quality assessment on the functionals of a protein should therefore be a better prognosis for native quality, and if the connectivity assumption is reflected by primes then quality assessment from using this assumption should also lead to a better prognosis. Therefore gains in quality assessment prognosis from dividing protein structures into smaller fragments based on their connectivity should, at the least, corroborate the connectivity assumption for defining and segregating protein primes. 


\section{Method}

\subsection{Alignments}

\section{MSA generation}

Searching for sequences using linear sequences, and their profiles, has long been solved using dynamic programming. Advanced methods of homology, utilizing statistics such as hidden Markov models, have over the years been developed and perfected. Today, we can search for homologs on the assumption of sequence similarity, using a laptop with the latest sequence alignment softwares, finding most of the homologs of a query sequence within minutes in a database of millions of sequences.

By selecting the set of sequences forming the protein family or families associated with the query protein, one can then build a multiple sequence alignment (MSA) for these sequences. These MSAs is built by incrementally adding more sequences to the alignment by aligning each one to the query protein. Such a pairwise sequence alignment is a column by column alignment of the residues in two proteins, which is optimized to align similar residues with each other maximizing a similarity scoring. Usually the scoring is based upon the phenotypical mutation distance between residues, stemming from their chemical properties and observation in sets of well studied proteins with different sequences that form the same structure.

The top, strongest protein homologue hits in an MSA are taken as having roughly the same structure and function as the query protein. MSA's are sorted by the score or significance estimation of each hit, yielding a list of protein hits and their alignments to the query, with significance and score decreasing from top to bottom. A threshold is usually imposed upon the process of collecting protein homologues, and proteins scoring an optimal alignment below this threshold is not even listed in the resulting alignment. Therefore, since strong homology as in sequence identity and similarity is known to mean that the structure of the proteins formed by 


\section{METHOD}

the sequences have the same characteristics and function, the top scoring matches are inferred to be homologous in structure and function as well as sequence.

An initial MSA is generated by generating a pairwise alignment for each protein in a database against the query protein using dynamical programming. The process of generating an MSA is done generally by selecting a query sequence as the origin, and then identifying all significant pairwise matches to this query according to some model. A standard model is to score these pairwise matches by the grade of identity of the matching residues and the number of inserts and deletions along the alignment. Indels, i.e. inserts and deletions, is a conceptual product of this alignment process when allowing for insertion of gaps in both the query and the hit sequences, in order to maximize the score of matching similar or identical residues to each other. Each indel is scored as a penalty, which balances the amount of indels to the resulting number of matches and the grade of their similarities in the alignment. The identification of the alignment that maximizes the score is done through the use of the standard method of dynamical programming.

Thereafter these pairwise alignments are sorted according to their scoring, and those proteins whose alignments to the query are scoring over a set threshold are then selected for building a hidden Markov model (HMM). The procedure is then repeated, but using the HMM as a query instead of the original query protein. Aligning an HMM to the database is done using the viterbi algorithm, which is in its essence an implementation of dynamical programming but for first order HMM.

The procedure of searching for sequences using the HMM profile and viterbi and then generating a new HMM is repeated either for a set number of times or until the MSA converges or drifts. One usually defines the convergence of an MSA as the point where either not many new sequences are found, after an iteration when comparing to the previous iteration, whilst drift is characterized by large losses of sequences in the later iterations that were present in the earlier iterations. Some drift is acceptable since that allows the HMM to naturally find a local minima in the sequence conservation well which the query protein is best associated with, but a too large drift means that the HMM might have found another more global minima related to another function. In the work of this thesis a fixed number of maximum iterations was preferred.

Depending on what the MSA will be used for, this drift of the selected sequences can be considered detrimental or beneficial and even encouraged. In the cases of softwares applying progressive alignments the aim is to discover a protein family and not only the sequences closest in functional relation to the protein query. The progressive alignment algorithms encourage drift and have adaptive models that utilize it in order to help identify distant homologs.

Further analysis can then applied to the resulting MSA, identifying conservation patterns or inferring residue interaction networks using epistatic modeling based on residue mutational covariance.

The multiple sequence alignments in this thesis is mainly generated using the computer software program jackhammer in the software suite HMMer. This has proven to produces more sequences in MSAs of adequate quality than from other comparative methods, for the subsequent analysis of conservation and covariances, taking into account that up to date databases are readily available for sequence searches.

MSAs should be treated as a protein subset of the general protein family discovered around, or in the vicinity of, the query protein. As MSAs produced in this iterative way are biased towards the particular function and position of the query sequence on the manifold of possible sequences, the MSA can be perceived to be sort of "trapped" in the area of sequences that are not evolutionary too distant from the origin query. 
To capture distant and convergently evolved protein homologs, or describing the function of the protein in terms of its interaction network, epistatic information needs to be considered in addition to sequence similarity patterns. The homology by sequence identity can be captured by using a linear sequence profile, but to study the homologs stemming from long term divergent or convergent evolution one need a method to capture and consider the compensatory mutational patterns, i.e. the epistatic model of the protein. One method for capturing these epistatic patterns are based around the idea of sequence covariance, or mutational correlation of residues in a protein family.

The problem in finding sequence homologs using the second order assumption of compensatory mutations has yet to be solved. While there are extant methods that try to do something along these lines, these are limited by two main reasons. First, the methods still use approximations, as the full description of the alignment problem using second order information is not yet complete. Secondly they also suffer due to the computational complexity, which grows much faster with the size of the proteins than what it does for the linear sequence homology assumption. In order to utilize both the first and second order assumptions in tandem, one would need to marry the concepts of dynamical programming and graph isomorphism or find another route entirely that combines the essence of the two.

\section{Entropy and conservation}

Sequence conservation of protein residues is a common measure for each residues relative importance for the functional integrity of the protein considered. This importance is defined as the evolutionary pressure to keep various parts of the protein relatively unperturbed and functionally intact as gauged over the protein family of the protein considered. When the conservation has been measured across the protein family it establishes a profile where patterns of conservation can be seen. Such conservation patterns signify which parts of each protein that are most important to the general function of the proteins of the family as a whole.

Sequence conservation is commonly measured across an MSA of a protein family and indicate which parts of the proteins therein that are important to the proteins function. A column of an MSA, representing a residue position in a protein family, is considered to be conserved when it is not deleted and prevalently assumes an identity within a small subset of all possible amino acid types. This assumption stems from the argument that if proteins with the same function has these residues within a narrow set of amino acids, then the proteins functions depend more critically on these residues and their identity than other residues with wider identity sets. Therefore the residue is completely conserved if it is only represented by one amino acid type throughout the whole MSA and it is completely non-conserved if it has the same amino acid frequency as the background or, in the ideal case, assumes every amino acid equally often.

One caveat of this approach, being focused on conservation, is that the actual selection of which proteins that are functionally related is done through using conservation measures, an approach that misses higher order information such as epistasis. This use of conservation measures in the definition of functional sameness is based upon statistical significance of sequence similarity or identity. As statistical significance requires that the selected set of homologs must have some sequence identity consensus, otherwise it would not be significant when using the sequence similarity measures, one is therefore actually selecting subsets of proteins which already has a common denominator that is centered upon a conserved pattern of amino acid over a specific region of the MSA profile. After selecting such a subset of sequences, by their consensus conservation profile as common denominator, and defining this conservation homology as a functional relationship, it is trivial to assume the inverse; i.e. that sequence conservation is the determinant of protein function and structural importance of individual residues. Albeit a half-truth, it is not the case in a general sense since it can only find the common denominators of a subset of the functionally related proteins, i.e. the subset found by having a first order sequence identity consensus as common denominator. In order to find a more complete set of protein functional homologs 


\section{METHOD}

one has to take into account higher order intra-sequence relationships between residues such as epistasis. There are relationships of even higher order than epistasis, one of which is part of this thesis as the concept of protein primes, but that subject is merely outlined for the purpose of further investigations. However, the concept of epistasis and how it relates to different modes of evolution, showing how standard conservation measures risks to fail in identifying specific functionality, will be discussed in more detail later in this thesis.

A measure that reflect this pattern of conservation and non-conservation is Shannon entropy from information theory [120], which is loosely based on the same assumptions as energy and entropy in physics. This measure of entropy assumes its maximum on any given residue column when all possible amino acids are equally prevalent over that column in the MSA, and its minimum when a column only assume a single amino acid throughout. Thus the measure of Shannon entropy is the negation of the desired measure of conservation and it is therefore subtracted from the theoretical maximum. The entropy is measured in Bits but can be converted into a conservation measure between zero and one through simple normalization with the theoretical maximum of Bits. [36]

The conservation, in the form of the inverse of the entropy, gives for a HMM of a multiple sequence alignment a measure of the importance of that positions residual identity and existence. That is to say how important the residue position is and that it is not a gap, i.e. that the sequence has a corresponding residue here - in order to be a member of the family that correspond to the particular function or structure that the family represents. Thus one can use conservation as a way to gauge the relative importance of parts of a protein sequence, with regard to its function, as gauged by the sequence variation in its protein sequence family, which is a measure of the evolutionary importance of those regions.

Shannon entropy is arguably the most wide spread and accepted measure of sequence conservation implemented in popular softwares. Examples of such softwares are the sequence alignment tools BLAST [7] and the HMMer suite [46], not to forget mentioning the HMM profile online conservation measurement service SkyLign [140].

These conservation patters, regions with low conservation interleaved by regions of high conservation, can be used as a telltale for domain boundaries. However, it is not always possible to tell from this information alone which domain each high conservation segment belongs to; as they could be from separate domains or stem from the same domain. These sequence segment interactions depend on how the structural fold of the protein is constituted, which is an unknown if we only know the protein family's sequence conservation patterns. To understand the segment connectivity we would either have to know the structure of the protein or have a model for the higher order relationships of the residues involved, such as epistasis.

Both structural and epistatic models can be expressed as undirected graphs with the residues taken as nodes and their interactions as edges. But as the conservation measure over a MSA only give us the importance of each residue position relative to each other, and their linear connectivity, we need other methods to probe the residual interconnectivity.

\subsection{Contact prediction}

The idea of co-evolution of protein residue pairs (Figure 3.1, and their use in deciphering protein structure and function has been around since the turn of the millennia. [88] Over the last decade, improvements in mitigating phylogenic bias [44] and discriminating between direct and indirect interaction predictions [93, 21, 70, 47, has greatly improved the sensitivity of the methodology.

Initially, efforts was made in using measures such as mutual information, based on entropy measures. 88 For these methodologies to report reliant residue interaction predictions one 


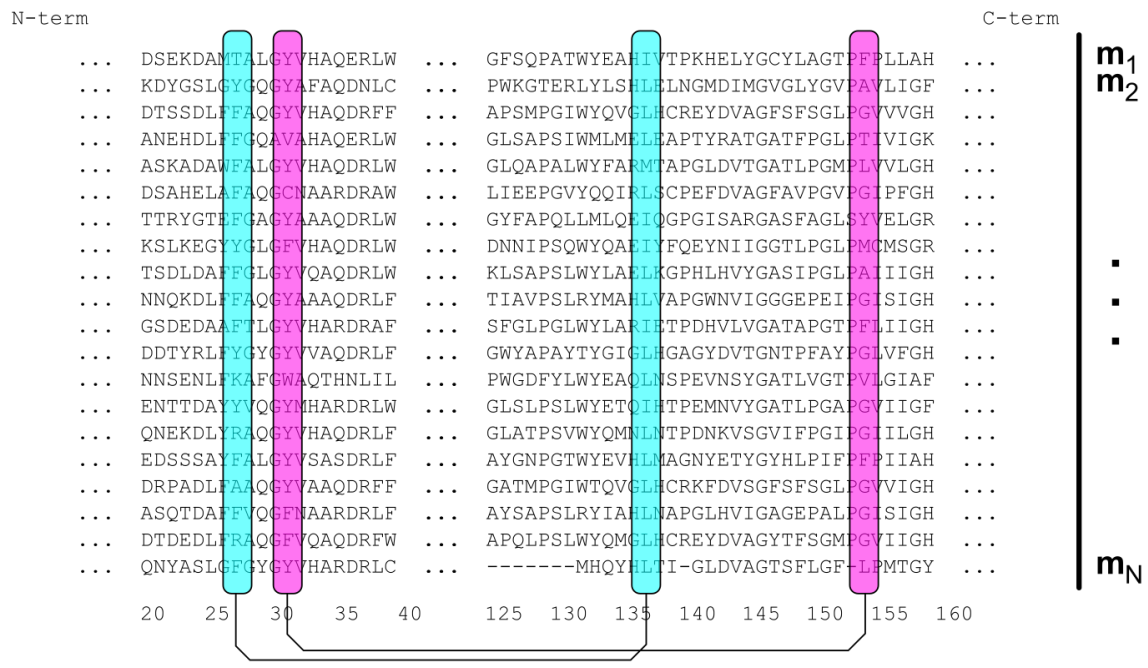

$$
\left\{R_{1}, R_{2}, \ldots, R_{L}\right\} \subset|\mathbb{Z}|_{<q}^{N}
$$

Figure 3.1: Co-evolution, co-adaptation, evolutionary couplings and direct contacts are all gauged as variants of analysis on co-occurrence of amino acid types in columns of multiple sequence alignments. Here, a snipplet of an MSA is shown for illustrative purposes, highlighting two selected pairs of columns. These can be represented as vectors in $|\mathbb{Z}|_{<q}^{N}$ space, by encoding each character in the column as a number from 0 to $q-1$, for $q$ different kinds of amino acids in the amino acid alphabet. A weight is often attributed to each sequence sample ( $m_{i}{ }^{\prime}$ s to the right) which reflects how prevalent/conserved the sequence is in the protein family.

need large high-quality sequence alignments, which was not abundantly available at the time, and generally the methods themselves was too computationally heavy.

A major hurdle that had to be overcome was the phylogenic bias. This bias comes into play due to the very nature of the MSAs used in computation of epistatic models in general; as mentioned, they are collected with the measure of conservation in mind, which will limit the patterns of covariation to that of a subset of proteins related to a conserved structure and function. In this setting, such conservation selects for the part of the phylogenic tree of sequences close to the query sequence. The solution to this problem of bias is twofold; first the conservation is normalized by both down weighing each sequences contribution in the data source depending on a measure of how many near neighbors within the phylogenetic tree it has. Then secondly, the significance of the artificial co-occurrence of conserved residues, that appear in the parameter solution of the model, are down weighed by their average product correction (APC). [44

It can be argued that given perfect data, one would not need the APC term, since then the regulation would favour a perfect model where all indirect interactions is reduced to the perfect 
a

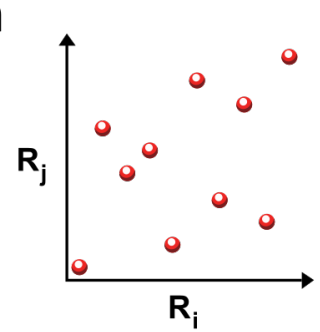

b $\left\{R_{i} \rightarrow\left\{R_{i 1}, R_{i 2}, \ldots, R_{i q}\right\} \subset \mathbb{B}^{N}\right.$

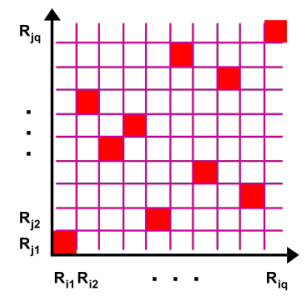

C

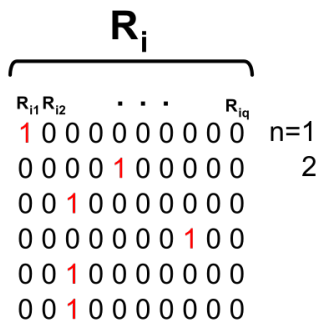

0010000000

1000000000

0000000100

1000000000

1000000000

0000100000

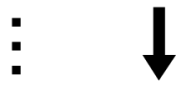

$0000000010 \quad \mathrm{~N}$

Figure 3.2: One hot expansion of the coordinate space into more dimensions, reducing the encoding to binary entries. (a) The residues are expanded into residue acids (b) which is an abstraction of the encoding of the data vectors into a matrix space. This forces all possible correlations to be linear or nonexistent. (c) Visualization of the one-hot encoding of one residue column in the MSA as $q$ columns of binary vectors, out of which only one entry is nonzero per row. This expands the actual matrix (S) into a 4-tensor, but in the implementations this is usually represented as a block matrix.

descriptor of each chain of direct interactions that couple the indirect covariates. But in reality, we almost always have less than perfect data, and alas we are left with scoring schemes like APC to help us in coping with this bias that comes out of the lack of complete data.

Since 2011 and onwards, there has been considerable development in the field of the prediction of epistatic protein graph models from protein sequence alignments alone. 21, 93, 70, 47, 33. It was largely due to a novel approach, treating each residues possible amino acid type as a separate node in the graph model, that the methods was made more effective and able to use sparser data, rendering predictions feasible. [139.

This novel idea means to expand the dimensionality of the problem from the space of protein residues into the space of residue acids. The expansion results in that a model with an $L$ residue long protein, using a amino acid alphabet of $q$ unique entities, becomes an expanded model with $L q$ residue acids. Thus the model ends up with $q$ times the initial number of nodes, see Figure 3.2

The dimensionality expansion is similar in nature to that of one-hot encoding [14] often employed in machine learning, and it maps the multinomial integer variables of protein residue amino acid identities into a boolean space (although there is no such defined concept in formal mathematics) of dimensionality $q$, which is equivalent to an integer space $\mathbb{Z}^{q}$ with all dimensions constrained to the interval $[0,1]$, denoted as $\mathbb{B}^{q}$. 
$\mathbf{a}$

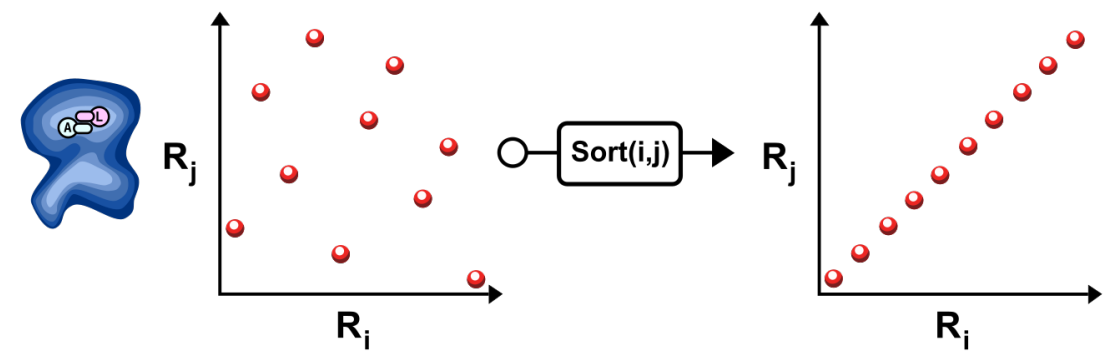

b

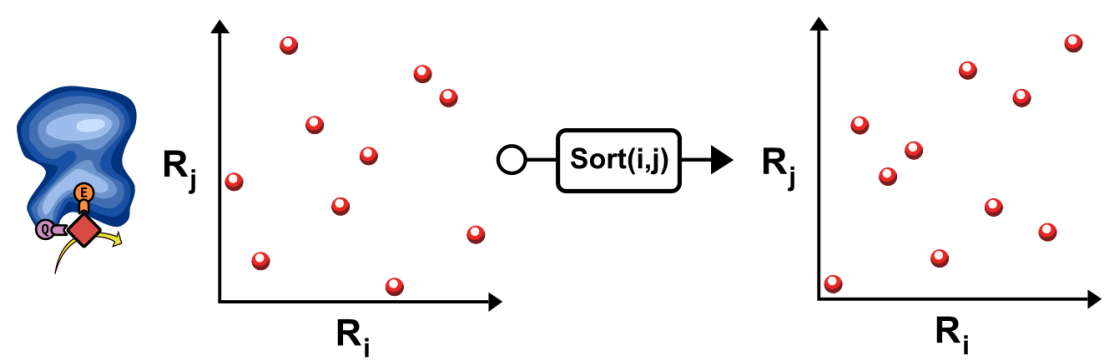

Figure 3.3: Simple example of one aspect of non-linear complexities arising from different environments and interactions surrounding the involved residues in any coupling. (a) A residue pair in a hydrophobic environment is expected to have one pattern of covariation, whilst a pair involved in catalysis on the surface of the protein $(\mathbf{b})$ is expected to have another. Thus if both are expressed over the same dimensions with $q$ states each, there is no single way of sorting the amino acids for both correlations to become linear.

In the boolean space so defined, a protein sequence sample is represented as a vector $v \in \mathbb{B}^{L q}$, and its outer product $v v^{\prime}$ yields a co-occurrence matrix sample.

It can seem contradictory at first that this would improve the model, since it expands the parameter space of a second order model with a factor of $q^{2}$, but it really simplifies the possible statistical behavior of the model (Figure 3.3). The statistical behavior is much more complicated for a residue node, having $q$ possible states (the full alphabet), as compared to a residue acid node which only has 2 possible states (either being present or not). Such complexity arises due to that standard statistical measures such as correlation can only capture linear relationships between nodes. This is no surprise as each residue node is in fact expected to have a non-linear relationship when it comes to its epistasis with other residues. When the same relationship is expanded into $q^{2}$ different relationships, each one of them is simplified into a linear relationship for which simple standard measures of statistics such as correlation becomes a complete descriptor of the relationship.

Learning a graph model in this expanded parameter space can then be effectively solved with standard methods of regulated optimization that favor parameter sparsity [90, 57] using linear algebraic solutions thanks to the models now linear behavior. 


\section{METHOD}

Once such a graph model has been extracted by learning its parameters using any of the proposed optimization schemes, one can then identify strong couplings between residues in the model as functional interactions between residues in a, possibly yet unknown, fold or structure. In order to make the inference from the model parameters to residue interactions, one first need to collapse the expanded tensor structure of the model, down into residue space again. This process is commonly handled by taking the Frobenius norm of each block matrix, yielded by treating each set of $q \times q$ residue acids in the model pertaining to an ordered tuple of nodes $(i, j)$ as a block, which collapse the tensor structure $\in \mathbb{R}^{L \times L \times q \times q}$ into a standard matrix $\in \mathbb{R}^{L \times L}$ . The resulting matrix can then be subject to further filtering for false positives, after which the resulting matrix is considered the Direct Contact [93] (DC), or Evolutionary Coupling [67] (EC), matrix. Furthermore, this kind of information about functional couplings between residues, be it either from DC's, protein dynamics simulation data analysis [119] or SCA [62], can deliver a clearer picture of a known but unintelligible structure. By seeing what is actually functionally coupled by evolutionary mandate will help elucidate which bonds between the residues that are of functional importance. Thereby interactions of the structure that do not have a direct functional coupling themselves can be viewed secondary in importance and the structural complexity can be greatly reduced in analysis without fear of loosing important features.

There is an intrinsical link between that of co-adaptation/epistasis and that of protein functional allostery. [112] 95] This is to say that an analysis of connectivity done on the epistatic model of a protein will reflect actual function and thus allosteric pathways in a protein. As such, given enough data to back an epistatic model of a protein of known, or unknown structure, one could resort to study the allostery on the level of epistasis instead of on trajectories of protein dynamics simulation data.

In order to understand the process of molecular evolution we need to ask ourselves - what is value to life? The simplest and yet arguably the most plausible answer, without going into theology or esoterica, is that it is merely to perpetuate itself; through the connectedness of the network of interactors that constitute life, a cycle of feedback that "eats" energy from outside as input and is able to perpetuate itself by finding even more energy to feed into itself. So, therefore, what is functional to life, is what is useful for life, which is what it values, i.e. being alive. Thus function is defined by the fact that it is keeping us alive, and what is keeping us alive is the perpetuation of this cycle. Therefore, the interactions within the cycle, between each of the counterparts of the cycle, is defining the function of the very same counterparts of the cycle.

This constitutes a link from DC's to that of protein structure, allostery and function, which rises out of the way evolutionary pressure has left traces of co-adaptation in the sequence databases (Figure 3.4. The cells of all lifeforms (but a few synthetic ones) has survived from generation to generation over the eons through a never ending cyclic mechanistic loop maintained by molecular networks of proteins and other macro molecules. Those individuals that had died are either a subject to circumstances or a mutant for which this network has deviated too far from that which, as a whole, is integrated enough to sustain the individuals life long enough for its progeny to proliferate. Given enough size population, a number of the former individuals strain will still survive and its corresponding hallmark mutations will mutate further and have a probability to be observed in current days databases, whilst the latter one with its inability to survive until maturation and proliferation will not. What we observe in the current day databases are not the exact copies of all of these individuals that has survived, but rather a jumble of all those mutations that comes from all those individuals that over the long time of evolution has evolved into the current day species. These current day species carry a combination of all of the most adaptable and efficient traits history has come up with so far.

That these evolutionary couplings arise in the first place mandates that they are somehow related to the function that provides some sort of evolutionary fitness for the species. Therefore this translates into an evolutionary pressure to keep the interactions that maintain the function and the residue interaction network that it represents, which has been discussed extensively in Section 1.3 
I

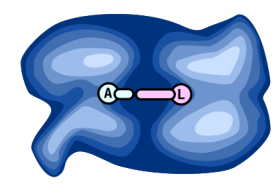

III

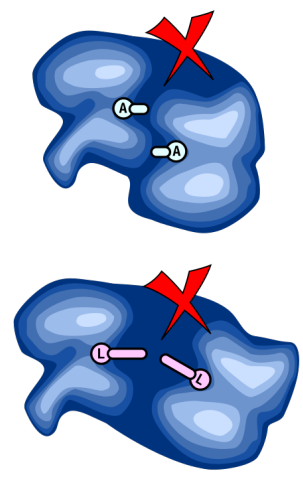

SELECTION
IV

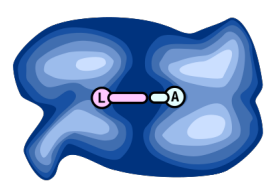

OBSERVED

(IN DATABASES)

$X X X A x X X L X X X$

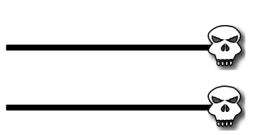

III $x x x L x x x L x x x$

IV $x x x \operatorname{Lxx} \times \operatorname{Axxx}$

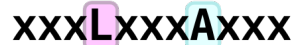

Figure 3.4: Illustration of mutations of a protein system constituted out of one protein highlighting a particular internal interface essential for proper function. (I) At first the protein is in a stable state, thanks to its amino acid composition. During an extended period of relaxation, when the protein system is not needed for the survival of individuals carrying it, a number of mutations accumulate within the population generating variant strains (II, III and IV). When the protein function is suddenly required due to a selection event, the two strains that have a faulty interface perishes (II and III), leaving us with only the two strains carrying intact interfaces to be observed in the present time (I and IV). When querying our sequence databases for homologous sequences, with either the sequence pertaining to I, IV or some other related sequence, we will thus only find I and IV, not the sequences II or III since they have gone extinct and are not present in the databases. This bias is caused by the fact that the content of our current day sequence databases reflect the currently available fauna of earth, since it is here and on living organisms that we have performed most sequencing experiments. Thus, the combinations of amino acid patterns that we can observe for this particular interface becomes constrained to those of I and IV, leaving combinations II and III out of the mix. It is this process that leaves traces of allowed patterns of amino acid combinations in our sequence databases; patterns that can be detected using co-occurrence modeling. 


\section{METHOD}

Moving into the next section, protein structure prediction will be discussed. In the structure prediction methods it is possible to guide the simulation using known epistatic couplings extracted in the above described methods of contact prediction.

\subsection{Structure prediction}

\section{Threading}

Protein threading is an approach where a protein sequence of an unknown structure is modeled by threading it through the backbone of known structures. A backbone conformation within such a structural template is accepted as a model for a stretch of the query protein sequence if it "fits" adequately well, considering some scoring function for bond angles and side chain similarities and other factors. The main source of structural templates for threading is usually provided by the Protein Data Bank (PDB), which is an online resource containing most of our known and experimentally backed models of protein structures and ensembles.

A well studied system of molecular conformation prediction software utilizing the threading method is the Rosetta suite which was based on the once novel idea to thread using template fragments. In Rosetta, the query protein sequence being modeled is threaded through short peptides of known structure en masse, optimizing a knowledge based energy function that minimize steric clashes within the predicted conformation while at the same time maximizing the structural homology by a threading fit score.

\section{Rosetta and FlexPepDock}

The Rosetta framework is built around a core set of procedures, which can be extensibly modified and combined by method developers to tailor protocols for specific folding and modeling tasks. A well defined protocol is documented, benchmarked and published in a peer review journal, rendering it available for other researchers to employ in their studies.

One such protocol is the Rosetta FlexPepDock [111] which was developed to dock flexible short peptide fragments (10-30 amino acids long) to a relatively fixed receptor molecule of known structure. In the FlexPepDock protocol, the receptor molecule is kept rigid while the peptide ligand is moved in a random fashion around the receptor, while allowing random perturbations of the ligand's backbone. The side chain orientations for both the receptor and ligand are optimized on the fly, keeping their backbones fixed, yielding a side chain conformation of approximately the lowest energy given the backbone conformation. This enables the derivation of a pseudo energy for any given receptor-ligand interaction determined by the conformation of the ligand backbone.

A Monte Carlo simulation is employed to find the receptor-ligand orientation and ligand backbone conformation of lowest energy. The conformation of the ligand backbone and the ligands position with relation to the receptor molecule is perturbed in a random fashion, measuring and storing the lowest energy conformations found. A great number of iterations are run, starting with a high simulation energy tolerance, or temperature, which is lowered towards the end of the simulation. The simulation temperature determines the likelihood to accept conformations of higher energy in order to avoid getting stuck in low-energy local minima, opting for finding the global energy minimum which is expected to be resembling the native state. As the simulation arrives at a relatively stable minima, i.e. it cannot find any better conformations with lower energy, the lowest energy conformation yet found is accepted as a putative near native state of the interaction and reported as a decoy; i.e. a sample structure.

As a best practice, a multitude of such Monte Carlo simulations are run in parallel, from multiple starting conformations which enables a more full probing of the ligand-receptor systems 
pseudo energy landscape. In doing so, one can utilize massive parallelization capabilities at super computing facilities such as the Swedish National Supercomputing Center (NSC), and avoid simulation bias by resorting to multiple starting conformations.

Clustering of the resulting decoys can then be employed to find the most plausible native-like conformation. It has been observed that clustering of low energy decoys reveals which such conformations that are the most prevalent in simulation. That a specific low-energy conformation is prevalent $i$ the simulation is a telltale of the conformation to be reminiscent of the native structure. [123

For a highly dynamic interaction, such as one that would be expected between a structured receptor and an IDP ligand, one would be better off in accepting an ensemble of such clusters as a putative model of the interaction. Such an ensemble cannot be directly interpreted as a statistical representation of the dynamical picture of the interaction, because of the way that such an ensemble has been generated; by only selecting the low energy conformations. In a real dynamical ensemble, also higher energy conformations are expected, but to an lesser extent than then low-energy conformations. This lack of high-energy conformations in a Rosetta generated ensemble makes it more reminiscent of a pruned ensemble, one in which high-energy states has been pruned away and only the low energy binding mode end-states (of sorts) are visualized.

\section{Simulation Constraints}

By introducing constraints to the simulation, one can steer the putative models to more reflect specific prior knowledge that are not represented by the general energy function of the simulation. Such prior knowledge could come from experimental data.

Experimental data collected by various means can be translated into, and expressed as, score terms between specific residues or atoms in the simulation. For an example, experimental data from differential HSQC NMR experiments express that, upon binding of a ligand, the chemical environments of certain residues in both receptor and ligand are being perturbed by the interaction. [66] These perturbations can then be translated into plausible ambiguous interaction constraints between perturbed ligand and receptor residues. One unbiased method of generating such ambiguous constraints are by assuming equal probability of interaction between any one of the perturbed interactants, forming a tuple set of interactions from the Cartesian product between the set of perturbed residues in the ligand and receptor respectively. [39] These tuples can then be assigned an interaction scoring function and weight, where the weight is a product of the mutual perturbation as measured in the experiment, and the scoring is a proximity function between pairs of residues in the simulation.

By adding such score terms derived from experiment to the general function of the simulation energy potential, one can then both use prior knowledge from protein structures in general, via the standard energy function of Rosetta, and the knowledge from new experiments by using the constraint function and weighted tuples. By making the terms pertaining to the experimental constraints score lower when the corresponding interaction residues are in proximity than when they are on a far distance, low-energy states will be skewed towards conformations that reflect that which is observed in experiment.

Some of the more interesting interactions do not form stable interactions, rendering direct interaction measures hard to achieve, and thus indirect measures of interactions can be employed instead. From NMR experiments, HNCO titration ratios can be measured, which reveals patterns of interactions through chemical shift perturbations (CSP) in NMR spectra influenced by factors such as nearness to specific chemical groups (amino acid side chains, surrounding water or other ligands) as well as the local orientation (phi/psi angles) of the protein backbone. 66

By assuming these indirect $\mathrm{HNCO}$ perturbations are due to direct interactions, between ligand and receptor in NMR titration experiments, one can transform these CSP into signals of interac- 


\section{METHOD}

tion that can be used in weighing constraints in simulation. By employing a three way experiment setup, where spectra of ligand and receptor is measured both separately and together, a difference spectra of CSP perturbations can be constructed.

The delta-CSP's are transformed into signals by first filtering them, using a standard approach [117], and then rescaling them into appropriate weights [66]. The rescaling is done relative to the strengths of the signals observed in experiment, yielding weights between 1 and 0 , i.e. between the maximum interaction signal and that of the filtered background. Thus weighted signals between 0 and 1 are generated for the most significant delta-CSP found in experiment.

These signals can then be used to form a weight for each interaction within a set of ambiguous constraint. By regarding the signals as a pseudo probability, ambiguous constraints can be readily formed by simple multiplication, yielding weights for the constraints as the product of the two individual signals, yet again between 0 and 1. This is then used in Rosetta as a scoring term, together with a scale parameter that will help balance the total contribution of experimental ambiguous constraint terms to the other Rosetta terms.

\subsection{Dynamics simulation}

Proteins do in nature exhibit some sort of dynamical behavior which manifests as a discretization of the systems behavior, with respect to time, in contradiction to an otherwise classically probabilistic and thermodynamical picture of chemical agents properties and interactions. Only by allowing an assumption of outcomes averaging on a macroscopic level, validates the description of a protein system in a simplistic classic thermodynamic view; since such a rough representation of the system as mere thermodynamical parameters renders the mechanistic description of the causation of complex interactions and pathways intractable. For a complete description of the interactions and mechanisms performed by real proteins, a more detailed model is needed than what is offered by entities in classic physical chemistry.

Since protein dynamics are manifesting on time and spatial scales demanding a resolution intractable to most feasible current day experimental methodologies, a typical resort is to use some sort of simulation in order to build mechanistic models of protein systems, filling in the gaps between the de facto experimental measurements.

As the dynamics of protein macromolecules and their complexes span from the detailed quantum mechanical scales of enzyme active sites to clock-work-like mechanics of domain movements, which are enormously complex to model using quantum models, it is common to use simplifying assumptions on the behavior of the protein chains to enable simulation within a consistent framework. Example of such a simplifying assumption is that of using a classical Newtonian model where forces and bonds between atoms are modeled with only classical concepts. A simplified view of the Newtonian assumption can be to imagine that the atoms are modeled as being held together with different kinds of springs, where each spring have a certain force equation depending on what kind of force it models. The Newtonian assumption is used in highly efficient dynamics packages such as GROMACS [23. 110], which comes with GPU enabled implementations [105] and an interface developed to fit high throughput computation such as those provided by super computing cluster environments [2].

Although packages like GROMACS support parallelization, the parallelization of a single simulation trajectory is limited due to the sequential nature of a dynamics simulation. A dynamics simulation is split up in epochs, i.e. time segments wherein forces are calculated and translated into momentum and coordinates are updated. Each epoch require that the epoch immediately previous is computed before itself can be computed, rendering the computation sequential in its temporal dimension and only parallel within epochs; i.e. we cannot compute the next step before we know the step immediately before it. Within an epoch the forces on each atom can be calculated and its coordinates and momentum updated in parallel by assuming that all other 
atoms are fixed during the epoch and minimizing errors by interpolation. Since the assumption within epochs does not hold between epochs, the parallelization is limited to the size of the system and long trajectories will have to be run more or less serially.

This limitation can be side-stepped and the employment of the full parallelization capabilities offered by super computing facilities can thus be achieved by performing computation of multiple shorter trajectories in parallel instead of only a single long one. If there are more compute resources available than there are room for parallelization, the dynamical behavior can be explored by starting multiple shorter trajectories from different starting conformations of the same system. This produces a more complete, yet shattered, picture of the dynamics of the system in shorter real time, thanks to the more efficient use of the compute resources. [30, 86]

In order to gain insight into what kind of dynamics that are present, statistical assumptions and models are needed to analyze such an ensemble of trajectories, produced by performing multiple shorter trajectory computations using different starting conformations, since the ensemble cannot be connected into a single trajectory deterministically. This thesis focus on such approaches of analyzing ensembles of parallel trajectories, by extracting condensed information about the principal dynamics illustrating the sensitivity of the dynamical behavior of the molecules to critical mutations.

\subsection{Clustering analysis}

[...] a universe comes into being when a space is severed or taken apart. The skin of a living organism cuts off an outside from an inside. So does the circumference of a circle in a plane. By tracing the way we represent such a severance, we can [...] begin to see how the familiar laws of our own experience follow inexorably from the original act of severance.

- G. Spencer Brown, Laws of Form 126

In silico modeling of protein structures carry with it a great uncertainty whether the resulting example structures (decoys) are native like and of any scientific value. A native like protein structural model is expected to be similar to a set of low energy protein conformations [28]. Thus it is reasonable to assume that any modeling software that can approximate the energy of generated conformations reasonably well, will produce most of its low energy conformations close to that of the native ensemble. Therefore, those low energy conformations that appear most frequently are also those that would be expected to be most similar to the native ensemble. 123

In this section we will discuss such clustering approaches with an emphasis on how to measure similarity, highlighting the problems that arise when one try to compare highly structurally mutable objects such as protein conformations.

\section{Structural clustering}

A complex structural ensemble model consisting of low energy decoys numbering in thousands can be readily reduced into a handful representative conformations by the use of a clustering approach, selecting the cluster centroids of the few largest conformational clusters as a representative set. It has been shown that the most prevalent low energy decoys, in the sense that they pertain to one of the larger low energy clusters, often reflect the native structure. [123] As such, it is natural to assume that such ensembles of centroids, from a handful of the largest lowest energy conformational clusters can be taken to represent an near native ensemble of, for an example, the true low energy binding mode of a receptor-ligand system. [28] This is especially true if using simulation constraints derived from experimental evidence, in such a way that the resulting decoys reflect such evidence. 


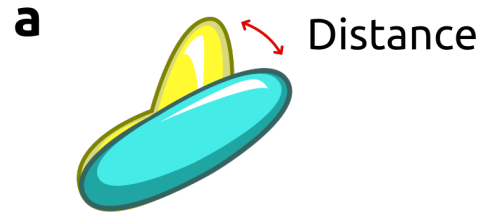

b

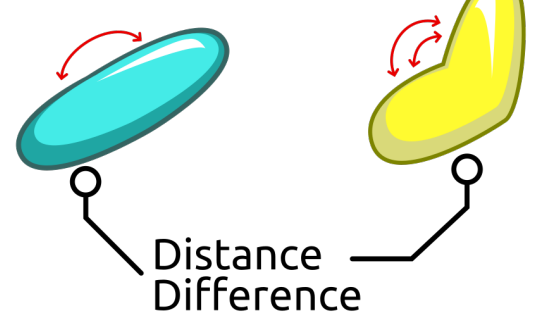

Figure 3.5: Illustration of conceptual difference between global and local similarity measures. Global similarity (a) measures the difference between the two conformations as a whole, given an optimal superposition, whilst local similarity measures (b) focus on the differences between internal configuration in a superposition independent manner.

Since there are as many methods of clustering as there are measures of entity similarities, as each measure of similarity naturally generates a partition of all entities so measured, this thesis focus on two popular methodologies targeting either similarity of the conformation with the protein taken as a whole or as in its separate parts. In order to describe a protein decoy's similarity to another, taken as a whole, a good approach is to consider the decoys as being immersed in a 3D-space only perturbed by rotation and translation transformations from that of other decoys. Such a setting consider decoys similar if there is little or no distance between the decoys' atoms given an optimal superposition (Figure 3.5 a). Superposition dependent measures are usually sensitive to small perturbations that accumulate through the structure, a sensitivity which tend to cause those measures to focus more on a global similarity between structures. The other way of describing similarity is by instead considering the decoys as immersed in an hyperspace induced by a measure of nearness between each and every residue, see Figure $3.5 \mathbf{b}$, where measures can be used that put emphasis on local structure as opposed to global structure.

In the next section, this difference between global and local similarity will be detailed with the perspective on a couple of similarity measures.

\section{Measures of Similarity}

Over time, it has been proven not to be easy finding measures of structural similarity in a way that put more emphasis on physiologically important structural regions and less so on less important regions. Initially, the Root-Mean-Square Deviation of atom positions (RMSD) has been the preferred measure of global similarity between molecular structures. [71] RMSD suffers from sensitivity to outliers, which in the case of proteins would result in that regions with the most variation between models will be contributing the most to the errors in the model. [145] Regions with most differences are often found in disordered or dynamic loop segments and 
C-/N-terminal tails, and since loops and dynamic elements are expected to be structurally mutable this results in error being falsely attributed to expected and natural variation. Instead, a focus on the differences between the models in the less varying parts are more important to judge their quality, since comparing the models on how well formed the naturally less mutable elements are will put emphasis on unnatural variation. This shifting of focus has proven not to be trivial as, within the continuum of variability in protein structure model ensembles, in attributing natural or unnatural variation through the measure of similarity there are no absolutes. Later developments has therefore resulted in a plethora of measures of structural similarity, as the bioinformatics field has progressed, each trying to address the weaknesses of RMSD and other measures discovered. [35]

Two major types of measures are of interest to this thesis; measures for gauging the similarity between structures on a global level, and measures concerned with local similarity of the structures. On the local level of similarity, the focus has settled upon comparison of local topology between protein models by converting them into graph models using inverse distance measures as similarity. Examples of measures that focus on local topological similarity using graph models are $\mathrm{S}_{\text {str }}$ /LGscore [81 35] and IDDT [87], which only depend on a proper alignment but are at least in part superposition independent. Other measures for global similarity are template modeling (TM) score [146], global distance test (GDT) and the local global alignment (LGA) [145], which depend on multiple global superpositions.

Again, RMSD is a measure that is calculated between two conformations of the same protein system, given a superposition of the two conformations, rendering a global measure of similarity. With such an superposition specified, the RMSD is just the mean of the sum of the squares of distance between each corresponding residue node in both structures when they have been superimposed in the same coordinate system. The square root of the sum of squares divided by the number of nodes is usually employed as a normalized measure of RMSD similarity.

Albeit its appealing simplicity, RMSD suffers from a sensitivity to outliers that makes the measure unable to differentiate between errors in domain folds or interfaces in multi-domain proteins. [145. 87] A high RMSD indicate significantly different structures with respect to a given optimal global superposition. This difference can arise from different kinds of factors. One factor are outliers, which in the setting of comparison of protein conformations is characterized by a few residues that are on markedly different coordinate positions while the rest of the two structures superimpose graciously. Such extreme deviations could arise in flexible linkers or $\mathrm{C}-/ \mathrm{N}-$ terminal tails. Another cause of these difference might come from that the two structures actually are folded slightly differently; many small perturbations add up to a large error. The last obvious major cause is that of domain misalignment, where a small difference in the interfaces translates into a large difference between the residues in one of the domains of the protein even though these corresponding two domains might be folded completely identically internally. Conceptually, this kind of misalignment is illustrated in Figure 3.5(a) where the kink in the yellow molecules represent a perturbed domain interface. The last two causes of high RMSD, the general misfolding and the interface error, are very different in nature but will produce the same kind of gradual RMSD error which is accumulated throughout the structure. The pattern similarity make the two errors hard to separate from each other using a global superposition dependent measure such as RMSD, and since the two errors have a marked difference in relevance it is of interest to have measures that can separate the errors, localizing and capturing the correct significance of the error.

\subsection{Essential Dynamics Analysis}

Simulations of protein dynamics in software suits such as GROMACS [23, 110] output data that need further analysis in order to enable the understanding of system dynamics in the wider sense. Dynamics simulations yield massive amounts of data in the form of trajectories. Tra- 


\section{METHOD}

jectories are ordered sets of protein conformations represented as a set of 3D coordinates, one for each atom present in the simulation. Except for an interesting show of moving molecules, direct visualization of such representations does not provide much insight. Therefore further exploration by various analysis techniques are often employed to extract the essential aspects of the data, thus reducing its complexity.

Pooling all conformations and performing a Principal Component Analysis (PCA) yields 3D vectors for the structure which can be used to visualize the principal movements of the system. The pooling is usually done by assimilating all simulation samples in the trajectories as a series of vectors. This vectorization is performed by putting each atom coordinate in a $3 \mathrm{~N}$ long vector, where $\mathrm{N}$ is the number of atoms in the simulation. Then by performing a PCA, as implemented by the Essential Dynamics Analysis (EDA) module in ProDy [19], one produces the vectors which represents the directions over which each atom vary the most over the course of the simulation. After superimposing the conformations, locating a central representative, the so acquired directional vectors can then be used to illustrate the directions of this principal movement for each atom as an arrow originating at the atoms corresponding center. This gives an ample indication of the overall character in the simulation with respect to the maximum structural variability, as viewed on the central representative conformation.

However, Essential dynamics does not provide any temporal information; the movements might be ratchet, or they may come in wider strokes and circular motions, not captured by the PCA.

At the same time as the lost temporal aspects might be seen as a loss of information; this indifference to temporarily and sequentiality renders EDA useful for analysis of a pool of trajectories stemming from multiple parallel simulations of the same protein system. Since there are no aspects of the temporal nature retained by the analysis, the interpretation should be roughly the same even from multiple starting positions. One caveat to this would be the case where these parallel isolated simulations are diverse in such a manner that they do simulate quite different conformational spaces, and that the pool do not represent the full conformational space of the protein system. In such an event the differences between the conformational spaces explored could skew the maximum variance in its favor. This skewing can then yield an artificial maximal dynamics variability that might not reflect that found in a larger simulation, exploring the full dynamical ensemble.

Any differences, between a couple of trajectories, in the picture of the EDA principal movement directions for the atoms around the central conformations will yield a hand waving about what can be the major dynamical differences between the flavor of the trajectories. Thus reducing the problem of comparing a full trajectory of movements, which essentially is a full movie of protein conformation, into the problem of differentiating between two conformations and the directional indications therein.

Furthermore, Essential Dynamics can give an overview over the conformational space explored by different trajectories, when performing the analysis over the pool of all trajectories and then projecting each individual trajectory on the largest components. This gives a low dimensional picture of the conformational distribution of the trajectory pool, as well as the contribution to the pool of each projected trajectory. [30] In this way, differences in simulation trajectories can be readily visualized, providing information of existent differences, albeit the details resulting in these differences are obfuscated.

Nevertheless, Essential Dynamics provide a great summary of the major dynamical aspects of the system explored, and renders comparison of protein systems of slightly different nature comparable. 


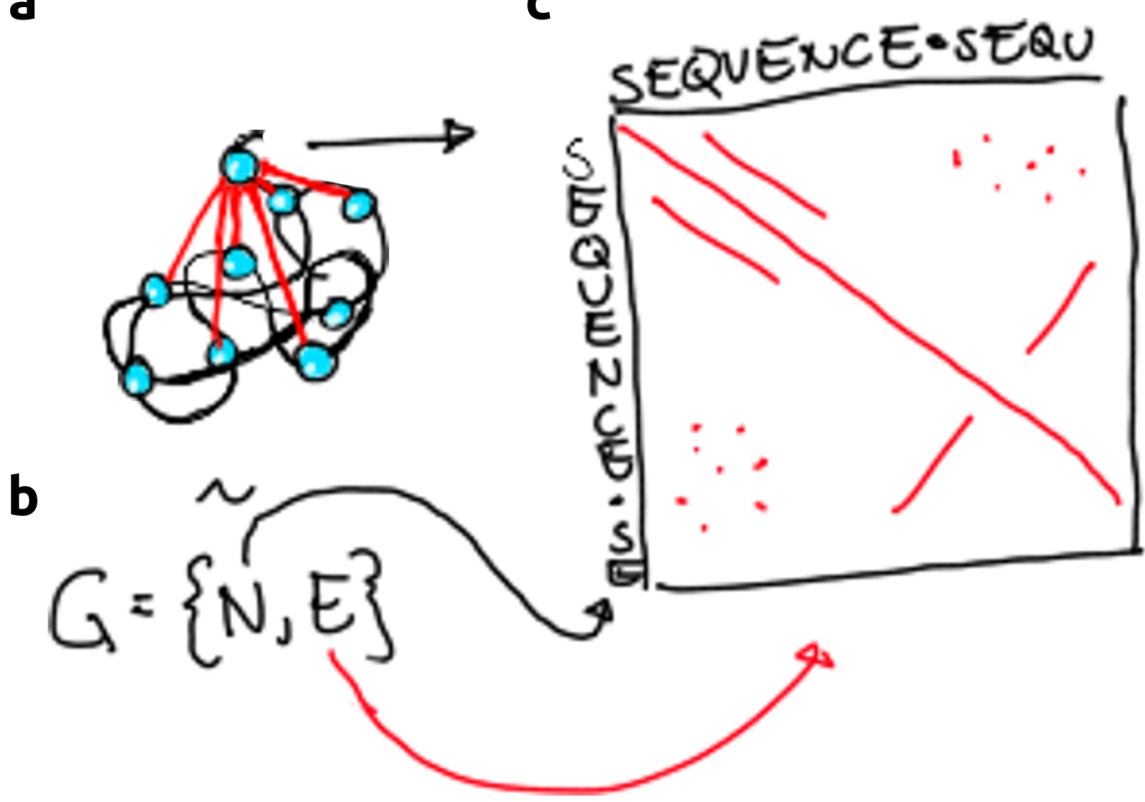

Figure 3.6: A sketch of a graph representation of a protein structure. The protein structures (a) residues are taken as the nodes with their distances as a parameter in calculating their edges. This is used in formulating the graph model (b), resulting in a matrix with the edges as entities and the node sequence along the rows and columns (c).

\subsection{Graphs and Structures}

Since this thesis has been quite rabid in proposing graph models to represent networks of various macromolecular interactions, it is only fitting that it also includes an actual section explaining these models to at least some detail. Therefore, a very short disposition on graphs pertaining to protein molecules follows below.

Network models from graph theory are well suited to model syntactic interactions in a semiquantitative way. These models had their principal inception in Euler's classic solution to the problem of the seven bridges of Köningsberg [50, 122]. The qualitative aspect of graph models is that they only consider the internal structure of the system, while they can model these internal relations relatively quantitative. In structural bioinformatics, the graph is built up by treating the protein residues as network nodes and applying a measure of similarity between them as the graph edges. The measures of similarity is often related to their spatial distance in 3D. In such a way, a protein graph can be expressed as a matrix containing the edge measures between each node and a sequence vector representing the nodes as shown in Figure 3.6

Graph tensors are extracted both from epistatic and structural models of this thesis, but their full potential is not explored before collapsing them back into flat matrices. The epistatic modeling softwares used in this thesis facilitate the extraction of tensors from multiple sequence alignments. These tensors are then flattened into matrices and treated as block matrices. The results 


\section{METHOD}

are also reported as a norm matrix produced from the norm of each block matrix. As the same is true for the tensors extracted for essential dynamics analysis (Sections 3.6 and 3.8 and ensemble quality assessment (Section 3.9) it would be interesting to see what could be explored by using some standard MATLAB libraries for tensor experimentation. [18]

However, the analysis of decoys from protein conformation prediction softwares, and the ensembles of trajectories from dynamics simulation softwares, necessitates models that enable objective and quantifiable comparison of protein conformations. A model, or decoy, of protein structures is usually depicted as a singular fixed conformation of the protein sequence fold in a 3D coordinate file. Such folds stored in coordinate files can be visualized using different softwares such as PyMOL and VMD. [38. 68] The model that represent a structure in 3D coordinates does not however lend itself to much analysis, except for as input to classical dynamics simulations using software such as GROMACS, as discussed previously. This is because such a model do only emphasize occupancy in space, and not relations of atoms to other atoms and thus the relationships between residues has to be computed and derived into another model wherein they are directly represented before any interaction analysis can be made. Usually this is done by computing a graph model out of the protein. As mentioned, these models are computed using the residues as the graph nodes, and applying a similarity function which is inverse proportional to the distance between residues, measured in some convenient way (Figure 3.6.

In this work, the similarity functions used are variations of the sigmoid thresholding logistic function, based on the distance as measured between each residues $\mathrm{C}_{\alpha}$-carbons. This ensures an emphasis on the backbone positions of the nodes, giving a slightly higher tendency for clusters of nodes to conceptually form following sequentially connected segments of structure.

There exists other interesting measures of nearness than simply distances; one could use the interaction surfaces as calculated from the interfaces of voronoi volumes around each residue. [100] Such a nearness function would yield a similarity matrix containing more emphasis towards the directionality and interactivity of the amino acid residues side chains than by using an inverse logistic measure of the distance between alpha carbons. This emphasis arises as the voronoi surface actually is directly affected by the side chain orientation and size, as compared to the carbon alphas which are not directly, but only indirectly, affected through the overall shape of the backbone.

For simplicity, as already mentioned, the work with protein graph models in the thesis has been on models derived from the measure of nearness based on a logistic of the inverse of the distances between the alpha carbons. Once such a graph model has been created, the similarity matrix represents for each element a measure of how proximal each residue are to each other; and the vector for similarity (i.e. row or column of the similarity matrix) represents how similar in interaction each residue is to each other with respect to the global graph model of the whole protein. In this way the measure defines the relationships within the molecules, and thus their intramolecular function with respect to each other.

Measures of similarity in function and interaction patterns can be used to infer interactive connectivity throughout the protein. Allostery can be conceived from it (see Section 1.3 , and functionally more or less independent subgraphs can be identified by partitioning the network, or similarity matrix, into chunks that are internally highly connected but have lower connectivity between each other. This give a better picture of which parts that are dependent on which other parts within the structure, and delivers a view of how interactions with other molecules or surfaces can be mediated indirectly through the networks of internal interactions. Interaction transferral from one part of the protein molecule to another part can thus be discovered, three dimensionally distant, which would otherwise not be visible from the study of the structure in 3D-coordinate representation only. 
Thus, graph models of proteins are an essential tool in understand the intramolecular dynamics and stability, as well as the different functional sites and their relationships; as simple 3-space coordinate representations fail to deliver this information in its raw form.

\section{Spectral Clustering}

Spectral clustering is a standard approach for partitioning nodes within a graph in a way that separate clusters that are denser in internal than external connections. [85] The clustering approach requires that a similarity matrix of the nodes can be formulated. After that it progresses through a reformulation of this similarity, or adjacency, matrix into a so called Laplacian matrix. Singular value decomposition is then performed on the Laplacian yielding a set of singular vectors and singular values. A special vector called the Fiedler vector is selected as the vector with the corresponding smallest, yet non-zero singular value. Each node represent an element on this vector, providing a value that can be used to sort the nodes. Such sorting results in that the nodes that are proximal to each other in this new sorting carry many high scoring similarities with each others, while those that are scoring low are distal. Over this induced dimension, it is easy to partition the set of nodes by a single threshold, yielding two sets of nodes. These two sets are the partitions that can be separated into two new graphs by minimizing the number of edges that are cut in the similarity matrix. [121]

\subsection{Allosteric Network Analysis}

Propensity for allostery can be indicated by residues being in proximal contact throughout the span of dynamics simulations allowing the identification of functional residue clusters. For allostery to occur between two residues via the structure-interaction-function route (see Section 1.3, the least requirement is that the two residues are in contact. When the two residues are in contact they can potentially mediate information from each other to any other residue they are also in contact with. Therefore is is of interest to study clusters of residues that maintain interaction throughout simulation trajectories as these can represent modular functional components of the structure.

WORDOM [119] is a software package that implements protein structure network (PSN) analysis supporting the identification of structural interaction clusters in dynamics trajectories. The PSN analysis starts by representing the frames of the trajectories as graphs. Frames are transformed into graphs by weighing each edge by the extent of nearness between each residue pairs side chains. These graphs can then be averaged over the trajectory yielding a general picture over the full simulation. Clustering of the nodes into communities then reveals structural interaction clusters which can be used to identify difference between simulations, functional relations between certain residues, and understand mechanical groups in the structure.

\subsection{Ensemble Quality Assessment}

In protein model quality assessment (QA), the problem is to find the best example of protein models that reflects the native structure of the protein under consideration, out of an ensemble of decoys. [48] Protein model decoys are examples of protein structural models for which the knowledge of the native structure is either unknown or withheld. Such decoys, pertaining to the sought after native structure, can be generated by any kind of software that produces protein model decoy sets. In order to identify the most native like decoy structure, the QA algorithm has some way of calculating a score for each decoy structure. These scores can be used to sort the decoys and thus find the highest scoring decoys. Given that the QA algorithm has been trained on a representative set of native structures, then the sorting rendered by the scoring can be trusted and the highest scoring decoy are probably one of the most native like ones. 


\section{METHOD}

The parity between ensemble and single model QA stems from how many decoys that are presented to the QA algorithm at the same time. In ensemble quality assessment, the full or a subset of the decoy set is presented at the same time. Therefore ensemble QA can be performed in a consensus fashion, which simplifies the problem since qualitative relational measures can be employed. Contrary to ensemble QA, in single model QA only a single decoy is presented to the QA algorithm, which has to produce a scoring based on only one observation at a time.

Next, the ensemble QA algorithm PCONS and its BiPART modification is discussed in brief.

\section{PCONS}

Contributed decoys in ensemble QA are expected to be of low-energy conformations, which enable the use of cluster centroids as good quality predictors. Structure decoy generating softwares often have their own internal QA algorithms evaluating produced and evolving conformations. As such, the reported final set of decoys usually have a conformation of reasonably low energy relative to that of random sampling. The decoy generation algorithm itself might also have a tendency to select structures closer together around easily realizable conformations. This affinity for easily accessible conformational space, together with low-energy reporting, enables clustering algorithms to detect low-energy decoys of easily accessible conformations within the reported decoys sets. Such centroids of accessible low-energy conformations are expected to be enriched in native like conformations. [123] By employing distance measures from decoy ensemble cluster centroids, QA algorithms can therefore blindly utilize also the accessibility criterion of the underlying decoy set generation softwares, improving their outcomes.

PCONS [137 is an ensemble QA algorithm that utilize this centroid effect, providing both a global and a local score for each structure in the ensemble. The PCONS QA algorithm uses the Kabsch algorithm to first superimpose each structure in the ensemble. Then it performs a round of calculating the local differences on each $\mathrm{C}_{\alpha}$-carbon in the ensemble, yielding an estimate of local quality over each residual position. [35, 60] Such estimation over the full superimposed ensemble has an averaging effect much like a comparison to a centroid structure. After the first round of estimation, a global score is calculated for each decoy from its local scores. The decoys can then be sorted by the global scores and intra-structural errors attributed via the local scoring.

\section{BiPART}

Domain-hinging motions and other large-scale perturbations in the conformations of the decoy set can give false error-attribution in ensemble QAs like PCONS. Superimposing structures with large-scale motions are problematic (see Section 3.5. Figure 3.5, risking large misalignments due to global motions. Error estimates in PCONS is based on the distances between residue atoms after superimposition. This, together with misalignments, leads to large error in estimates of local integrity in some domains due to their global motions. False errors in local integrity is then propagated into the global estimates which risks lowering the QA algorithm performance.

The BiPART algorithm aims to mitigate this error by partitioning the structure into two subdomains and then combining error estimates from the two separate partitions. By generating a graph model from each structure, BiPART populates a tensor model representing the ensemble of decoys. This tensor is then flattened down into an average graph model edge matrix of the interactions present in the decoy set. A min-cut approach is then employed to partition the residues into two set, via the well known method of spectral clustering. [85] These two residue sets are fed through PCONS together with the decoy set generating two separate domain QA reports. By combining the two reports the final global and local scores are delivered for the full decoy structures of the ensemble. 


\section{Present Investigations}

\subsection{Function in transiently ordered islets of intrinsically disordered proteins (Paper I)}

In this paper, specific function is attributed to one short protein prime/MoRFs-like segment termed MB0 of the intrinsically disordered protein c-MYC by joint efforts of investigation on a $\mathrm{N}$-terminal sequence of c-MYC encompassing both segments MB0 and MB1.

Two intrinsically disordered regions in the protein c-MYC owning MoRFs-like [136] character was investigated in complexing with its structured partner PIN1. Function was verified and attributed to small transiently ordered subsequences, or boxes (MoRFs-like), of the intrinsically disordered protein c-MYC. Empirical measurements was followed by constrained modeling yielding a view of the interaction. Mutations in key positions of proposed interaction rendered the function absent and thus the wild-type phenotype disappeared. Combined with empirical measurements indicating a lowering of the interaction prevalence/strength between c-MYC MB0 and Pin1 a corollary can be drawn that specific aspects of the total function of the c-MYC protein, namely those of proper interaction with the Pin1 protein, can be attributed to the MB0 region of c-MYC.

Here the computational method used was developed under an assumptions that is not true for this study; the method assumes that the structure of the protein peptide it is modeling is represented by some conformation found by a similar sequence in a folded globular domain within the PDB. This is not true, since there are no models of IDPs segments within the PDB dataset used to construct the fragment library for Rosetta. However, to mitigate this error, another assumption was made on the particular bound states of the sub-ensemble that is actively interacting through the full length of the MYC boxes. The assumption is that such bound states should owe more familiarity to some of those fragments in the method library. Then the experimental constraints is applied to modulate the energy function toward the empirical evidence and attain a plausible model for such bound states of the sub-ensemble. 
Interestingly, a multitude of binding modes is evident even in this very constrained subensemble. As such, it strongly suggests that this ensemble model presented, is a part of a fuzzy complex interaction [131, 58 between Pin1 and c-MYC; which is this thesis' contribution to the work.

\subsection{Functional analysis of protein structure dynamics using graph models and multivariate statistics (Paper II)}

Allostery prone networks of MexR is identified by PSN analysis in the WORDOM software. Comparing wt and R21W networks, there is a much more marked difference of allosteric prone interactions between networks than within networks. This can be seen as a collapse of the network prime structure into an inactive and constrained constellation. Activity of DNA binding is also predicted to be less frequent. Such alteration of allosteric prone networks interactions coupled to the loss of function illustrate the function by prime interaction pattern alteration.

The MexR protein is a DNA-binding regulator responsible in the bacterium Pseudomonas aeruginosa (P.a.) for repressing its native ability of multi-drug resistance (MDR) through the downregulation of the production of broad spectrum efflux pumps. The differences between a functional (MexR-wt) and non-functional (MexR-R21W) structure was discovered to be very minute. A crystal structure of a particularly MDR promoting mutant of MexR was determined by the coauthors. Through a network analysis [119] of MexR over data procured from extensive molecular dynamics simulations [110] it was understood that this difference was from a change in the interaction between separate functional elements of the wild-type structure, and not of alternations in internal interactions within any centrally important structural element. The alteration of the interaction introduced structural rigidity in mutant MexR-R21W, not present in MexR-wt, and as a result changed the function of the structural elements relative to each other due to the altered interfacial interaction between the elements. Thus the change in functional phenotype of the MexR constellation as a whole, can be attributed to the malfunction of the interfaces between the functional elements; i.e. the functional elements has changed their function relative each others, where function is interpreted as their interactions, and therefore the structure of mutant MexR loose its ability to fulfill its role as a MDR efflux-pump repressor.

\subsection{Improvements in Protein Model Quality Assessment from Automated Domain Partitioning using Spectral Clustering (Paper III and Manuscript I)}

Pseudo-primes is here identified using a bi-partitioning method based on spectral clustering over a set of low-energy decoys from protein structure prediction softwares; this divide and conquer approach improves decoy quality assessment which indicates that the pseudo-primes are a natural partition that reflect a functional role in the native protein structure.

In order to enable the study en masse of protein primes or functional sub-elements of structure, automatic methods for identification of primes are needed. Since protein primes are defined as smaller structural nuclei with denser interactions than over prime interfaces, these primes are expected to form energetically more stable cores than other randomly selected partitions of the structures. Therefore, predicted low-energy structural decoys from modeling software using any kind of pseudo-energy for optimization based on compactness and favorable interactions ought to promote prime formation. Given a set of low-energy decoys from a number of such modeling softwares, any dense structural kernels that can be found conformationally preserved throughout such a set of decoys should be indicative of a protein prime. Such a decoy set is constituted out of decoys of low-energy conformations, as predicted from many different softwares; as these softwares carry different pseudo-energy definitions, the kernel can be judged 
4.4. Functional Interaction Network of c-MYC Conserved Regions determined by Evolutionary Couplings (Manuscript II)

to be stable with respect to many evaluators and therefore taken to represent a dense core of low energy.

This paper explores a spectral clustering approach as a concretion to identifying such primes and their application in a divide and conquer approach to model quality assessment. It is shown that the decoy quality measure is improved on attributing errors of structural quality on the local level, when a simplistic BiPART pseudo-prime partitioner is employed together with the state-of-the art quality assessment tool PCONS, as compared to PCONS alone. Furthermore, the BiPART-PCONS methodology maintain a competitive quality assessment on the global level of the full decoy, as compared to PCONS and other tools alone. On the whole, it is shown that even with a very simplistic approach to identify only pseudo-primes, we are able to better assess decoy quality in some interesting and hard cases.

\subsection{Functional Interaction Network of c-MYC Conserved Regions determined by Evolutionary Couplings (Manuscript II)}

Protein primes are here used to describe MoRFs-like or slightly conserved regions. By sequence homology searches they are found in a multitude of protein constellations, forming separate families which only intersect partially. This implies that the primes are used in multiple different constellations, possibly functionally alien to each others as wholes.

The modular nature of the c-MYC N-terminal intrinsically disordered domain is investigated. Observation of small linear MoRFs-like [136] segments of sequence, termed boxes, which maintain higher sequence conservation than the surrounding linker regions between the boxes are used as queries in a number of sequence homology searches.

These boxes are hypothesized to be responsible to carry the specific interactive functionality of the IDP; and thus understanding of their interaction pattern can give information on function and pathology. Furthermore, interaction patterns between the boxes are indicative on their mutual interplay. By employing evolutionary coupling analysis on the separate boxes and between them, an interaction graph of the conserved residual network of c-MYC can be constructed. Such an interaction graph yield predictions on which boxes that are to be expected to be functionally related. Thus revealing interdependence between boxes for carrying out their role in the host.

Residual couplings emerge in the analysis, revealing functional patterns that are correlated with empirical evidence. 



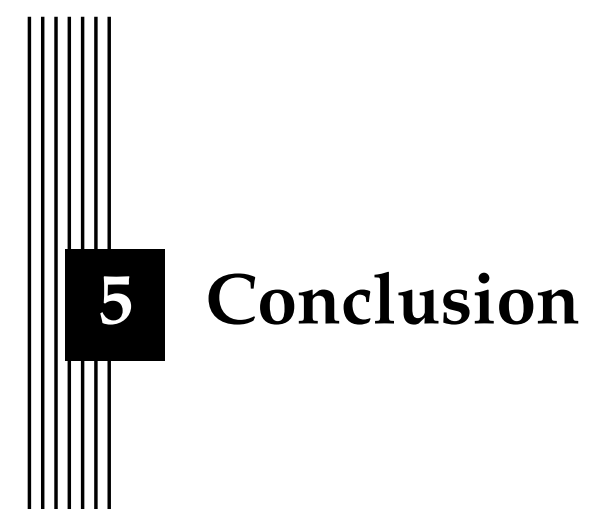

Evolution proceeds through one singular process, but it can be perceived differently on different scales. On the molecular level it can be described in the light of epistasis, whilst on the macroscopic level is best modeled via a relaxation-selection model.

Network models of residue, domain and protein interactions are today available and shed light on a myriad of problems, but they are limited in scope due to their inability to handle the connection between hierarchies.

Therefore it is of utmost importance to develop models that incorporate the interaction holarchy [74] systematically so that information can propagate up and down in the models, effectively cross-pollinating our knowledge of protein systems. Such data derived from cross hierarchical connections could prove invaluable in providing new insights to, and resolution of, previously unresolved problems in structure and systems biology.

Intrinsically disordered proteins emerge as candidates in great need for investigation with network models. They are hypothesized, feverishly so in the literature, to have a role both as functions derived from new mechanics other than structural interactions, and as vectors for fast promiscuous evolution through epistasis.

A central concept for function is warranted, which can capture and describe function arising from physical interactions, dynamics and the like. The epistatic view on function might be one of the most general ones, given that it capture both relationships stemming from dynamical events and from functional interactions. Further generalization can be realized as the protein prime and constellation model, enabling the expansion of the functional description into tensor spaces potentially supporting both conformational precession and environmental variability. 


\section{CONCLUSION}

As research into bioinformatics progresses, time will only tell how novel techniques such as epistasis based function analysis and new structure ensemble determination methodology such as cryo-EM ${ }^{1}$ will influence and develop the field.

${ }^{1}$ Electron microscopy is a widely applicable technique, shedding light on anything from mineral samples [51] to biological systems. In recent years the Cryo-EM technique has been conceived which utilize massive computational resources and smart algorithms to combine multiple low-resolution images in-silico into high-resolution image of X-ray crystallographic resolution. 


\section{\|\|\|\|\|\|$_{6}$ Future Perspectives}

This, too, will pass.

- Eckhart Tolle

When you, the reader, closes this little booklet, you will take with you the concept of protein primes. Not with the interpretation that interactions are function [5], neither that dynamics are function [115], nor epistasis is function [26]; you will bring it with you as the description of protein function centered between these views as a model accepting inferred functional connections from all of them.

But you do not stop there, constraining this idea of network connectivity to proteins; no, you expand the concept of networks as a model for all of life, letting it permeate the holarchy [74] wherein the protein prime model merely resides as an aspect of one level of clustering within its infinite fractal structure.

With this view of life, yourself and yourother, you continue your work of creation wherein you flow, fused together in perpetuity; celebrating existence as the everlasting tug between unity and partition.

In Lak'ech 



\section{Bibliography}

[1] Gregory A Petsko. "Dog eat dogma". In: Genome Biology 1 (July 2000), comment1002. ISSN: 1474-760X. DOI: $10.1186 / \mathrm{gb}-2000-1-2-$ comment1002 URL: https://doi.org/10.1186/gb-2000-12-comment 1002.

[2] Mark James Abraham, Teemu Murtola, Roland Schulz, Szilárd Páll, Jeremy C. Smith, Berk Hess, and Erik Lindahl. "GROMACS: High performance molecular simulations through multi-level parallelism from laptops to supercomputers". In: SoftwareX 1-2 (Sept. 2015), pp. 19-25. ISSN: 2352-7110. DOI:10.1016/j.softx.2015.06.001. URL: http: / / wWw. sciencedirect.com/science/article/ pii/S2352711015000059 (visited on 04/06/2018).

[3] Lateef Adewoye, Ainsley Sutherland, Ramakrishnan Srikumar, and Keith Poole. "The MexR Repressor of the mexAB-oprM Multidrug Efflux Operon in Pseudomonas aeruginosa: Characterization of $\mathrm{Mu}-$ tations Compromising Activity". en. In: Journal of Bacteriology 184.15 (Aug. 2002), pp. 4308-4312. ISSN: 0021-9193, 1098-5530. DOI: 10 . $1128 / J B .184 .15 .4308-4312.2002$, URL: http: / / jb.asm . org/content/184/15/4308.

[4] Vadim Alexandrov, Ursula Lehnert, Nathaniel Echols, Duncan Milburn, Donald Engelman, and Mark Gerstein. "Normal modes for predicting protein motions: A comprehensive database assessment and associated Web tool". en. In: Protein Science 14.3 (Mar. 2005), pp. 633643. ISSN: 1469-896X. DOI: $10.1110 /$ ps .04882105. URL: https: 
// onlinelibrary . wiley . com / doi / abs / 10 . 1110/ps . 04882105

[5] Patrick Aloy and Robert B. Russell. "Structural systems biology: modelling protein interactions". en. In: Nature Reviews Molecular Cell Biology 7.3 (Mar. 2006), pp. 188-197. ISSN: 1471-0080. DOI: 10 . $1038 /$ nrm1859. URL: https : / / www - nature . com / articles / nrm1859

[6] Patrick Aloy and Robert B. Russell. "Ten thousand interactions for the molecular biologist". en. In: Nature Biotechnology 22.10 (Oct. 2004), pp. 1317-1321. ISSN: 1546-1696. DOI: 10 . 1038 / nbt1018. URL: https://www.nature.com/articles/nbt1018.

[7] Stephen F. Altschul, Warren Gish, Webb Miller, Eugene W. Myers, and David J. Lipman. "Basic local alignment search tool". In: Journal of Molecular Biology 215.3 (Oct. 1990), pp. 403-410. ISSN: 00222836. DOI: 10 . 1016/S0022-2836(05) 80360-2 URL: http: / / wWw . sciencedirect . com / science / article / pii / S0022283605803602

[8] Vikram Alva, Johannes Söding, and Andrei N Lupas. "A vocabulary of ancient peptides at the origin of folded proteins". en. In: eLife 4 (Dec. 2015). ISSN: 2050-084X. DOI: 10 . 7554/eLife . 09410 . URL: http://elifesciences.org/lookup/doi/10.7554/elife. 09410.

[9] Madhanagopal Anandapadamanaban, Robert Pilstål, Cecilia Andresen, Jill Trewhella, Martin Moche, Björn Wallner, and Maria Sunnerhagen. "Mutation-Induced Population Shift in the MexR Conformational Ensemble Disengages DNA Binding: A Novel Mechanism for MarR Family Derepression". en. In: Structure 24.8 (Aug. 2016), pp. 1311-1321. ISSN: 09692126. DOI: $10.1016 / j$.str.2016. 06 . 008 . URL: http : / / linkinghub.elsevier. com / retrieve / pii/s0969212616301332 (visited on 04/11/2017).

[10] Cecilia Andresen, Sara Helander, Alexander Lemak, Christophe Farès, Veronika Csizmok, Jonas Carlsson, Linda Z Penn, Julie D Forman-Kay, Cheryl H Arrowsmith, Patrik Lundström, and Maria Sunnerhagen. "Transient structure and dynamics in the disordered c-Myc transactivation domain affect Bin1 binding." In: Nucleic acids research 40.13 (July 2012), pp. 6353-66. DOI: 10 . 1093/ nar/ gks263. URL: http : / / www - pubmedcentral . nih . gov / articlerender. fcgi ? artid $=3401448$ \& tool=pmcentrez \& rendertype=abstract 
Bibliography

[11] Cecilia Andrésen, Shah Jalal, Daniel Aili, Yi Wang, Sohidul Islam, Anngelica Jarl, Bo Liedberg, Bengt Wretlind, Lars-Göran Mårtensson, and Maria Sunnerhagen. "Critical biophysical properties in the Pseudomonas aeruginosa efflux gene regulator MexR are targeted by mutations conferring multidrug resistance". en. In: Protein Science 19.4 (Apr. 2010), pp. 680-692. ISSN: 1469-896X. DOI: $10.1002 /$ pro. 343. URL: https : / / onlinelibrary · wiley • com/doi/abs / 10 . $1002 /$ pro.343.

[12] Christian B. Anfinsen. "Principles that Govern the Folding of Protein Chains". en. In: Science 181.4096 (July 1973), pp. 223-230. ISSN: 00368075, 1095-9203. DOI: $10.1126 /$ science.181.4096.223. URL: http://science.sciencemag.org/content/181/4096/223.

[13] Anonymous. Emergentism. en. Page Version ID: 828400806. Mar. 2018. URL: https://en .wikipedia . org/w/index . php?title= Emergentism\&oldid=828400806 (visited on 03/20/2018).

[14] Anonymous. One-hot. en. Page Version ID: 808377328. Nov. 2017. URL: https://en.wikipedia.org/w/index.php?title=Onehot $\&$ oldid $=808377328$

[15] Anonymous. Vitalism. en. Page Version ID: 830263604. Mar. 2018. URL: https: / / en . wikipedia . org/w/index . php ?title $=$ Vitalism\&oldid=830263604 (visited on 03/20/2018).

[16] anonymous. Hmmer publications. Jan. 2017. URL: http : / / hmmer . org/publications.html (visited on 01/19/2017).

[17] Michael Ashburner, Catherine A. Ball, Judith A. Blake, David Botstein, Heather Butler, J. Michael Cherry, Allan P. Davis, Kara Dolinski, Selina S. Dwight, Janan T. Eppig, Midori A. Harris, David P. Hill, Laurie Issel-Tarver, Andrew Kasarskis, Suzanna Lewis, John C. Matese, Joel E. Richardson, Martin Ringwald, Gerald M. Rubin, and Gavin Sherlock. "Gene Ontology: tool for the unification of biology". In: Nature genetics 25.1 (May 2000), pp. 25-29. ISSN: 1061-4036. DOI: 10 . 1038/75556. URL:https://www.ncbi.nlm.nih.gov/pmc/ articles/PMC3037419/(visited on 10/05/2017).

[18] Brett W. Bader and Tamara G. Kolda. "Algorithm 862: MATLAB Tensor Classes for Fast Algorithm Prototyping". In: ACM Trans. Math. Softw. 32.4 (Dec. 2006), pp. 635-653. ISSN: 0098-3500. DOI:10.1145/ 1186785 .1186794, URL: http://doi . acm . org/10.1145/ 1186785.1186794

[19] Ahmet Bakan, Lidio M. Meireles, and Ivet Bahar. "ProDy: Protein Dynamics Inferred from Theory and Experiments". en. In: Bioinformatics 27.11 (June 2011), pp. 1575-1577. ISSN: 1367-4803. DOI: 10 . $1093 /$ bioinformatics/btr168, uRL:https://academic.oup.com/ bioinformatics/article/27/11/1575/217006 
[20] David Baker and Andrej Sali. "Protein Structure Prediction and Structural Genomics". en. In: Science 294.5540 (Oct. 2001), pp. 93-96. ISSN: 0036-8075, 1095-9203. DOI: 10 . 1126 / science . 1065659, URL: http://science.sciencemag.org/content/294/5540/93 (visited on $02 / 20 / 2018$ ).

[21] Sivaraman Balakrishnan, Hetunandan Kamisetty, Jaime G Carbonell, Su-In Lee, and Christopher James Langmead. "Learning generative models for protein fold families." In: Proteins 79.4 (Apr. 2011), pp. 1061-78. DOI: 10 . 1002 / prot . 22934. URL: http: / / www . ncbi.nlm.nih.gov/pubmed/21268112.

[22] Nenad Ban, Poul Nissen, Jeffrey Hansen, Peter B. Moore, and Thomas A. Steitz. "The Complete Atomic Structure of the Large Ribosomal Subunit at $2.4 \AA$ Resolution". en. In: Science 289.5481 (Aug. 2000), pp. 905-920. ISSN: 0036-8075, 1095-9203. DOI: $10.1126 /$ science. 289.5481.905. URL: http:// science.sciencemag.org/ content/289/5481/905.

[23] H.J.C. Berendsen, D. van der Spoel, and R. van Drunen. "GROMACS: A message-passing parallel molecular dynamics implementation". In: Computer Physics Communications 91.1-3 (Sept. 1995), pp. 43-56. DOI: 10.1016/0010-4655(95)00042-E. URL: http://linkinghub. elsevier.com/retrieve/pii/001046559500042E.

[24] Christian Berggren. Vetenskaplig publicering: historik, praktik och etik. Swedish. OCLC: 1016175821. Lund: Studentlitteratur AB, 2016. ISBN: 978-91-44-10884-1.

[25] Helen M Berman, John Westbrook, Zukang Feng, Gary Gilliland, T N Bhat, Helge Weissig, Ilya N Shindyalov, and Philip E Bourne. "The Protein Data Bank". In: Nucleic acids research 28.1 (2000), pp. 235-242. URL: wWw.rCsb.org

[26] Jeffrey I. Boucher, Daniel N. A. Bolon, and Dan S. Tawfik. "Quantifying and understanding the fitness effects of protein mutations: Laboratory versus nature". eng. In: Protein Science: A Publication of the Protein Society 25.7 (July 2016), pp. 1219-1226. ISSN: 1469-896X. DOI: $10.1002 /$ pro. 2928

[27] Celeste J. Brown, Sachiko Takayama, Andrew M. Campen, Pam Vise, Thomas W. Marshall, Christopher J. Oldfield, Christopher J. Williams, and A. Keith Dunker. "Evolutionary Rate Heterogeneity in Proteins with Long Disordered Regions". en. In: Journal of Molecular Evolution 55.1 (July 2002), pp. 104-110. ISSN: 0022-2844, 1432-1432. DOI: 10 . 1007/s00239-001-2309-6. URL: https : / / link. springer. com/article/10.1007/s00239-001-2309-6. 
Bibliography

[28] J. D. Bryngelson, J. N. Onuchic, N. D. Socci, and P. G. Wolynes. "Funnels, Pathways and the Energy Landscape of Protein Folding: A Synthesis". In: arXiv:chem-ph/9411008 (Nov. 1994). arXiv: chemph/9411008. URL: http: //arxiv.org/abs/chem-ph/9411008 (visited on $03 / 27 / 2018$ ).

[29] Zimei Bu and David J. E. Callaway. "Chapter 5 - Proteins MOVE! Protein dynamics and long-range allostery in cell signaling". In: Advances in Protein Chemistry and Structural Biology. Ed. by Rossen Donev. Vol. 83. Protein Structure and Diseases. Academic Press, Jan. 2011, pp. 163-221. DOI: $10.1016 /$ B978-0-12-381262-9.00005-7. URL: http: // wWw.sciencedirect.com/science/article/ pii/B9780123812629000057.

[30] Caves Leo S. D., Evanseck Jeffrey D., and Karplus Martin. "Locally accessible conformations of proteins: Multiple molecular dynamics simulations of crambin". In: Protein Science 7.3 (Dec. 2008), pp. 649-666. ISSN: 0961-8368. DOI: 10.1002 / pro.5560070314, URL: https: // onlinelibrary .wiley.com/doi/full/10.1002/pro. 5560070314 (visited on 04/06/2018).

[31] Ku-ming (Kevin) Chang. "Alchemy as Studies of Life and Matter: Reconsidering the Place of Vitalism in Early Modern Chymistry". In: Isis 102.2 (June 2011), pp. 322-329. ISSN: 0021-1753. DOI: 10 . $1086 /$ 660141. URL: https://www. journals . uchicago.edu/doi/ full/10.1086/660141 (visited on 03/20/2018).

[32] Thang K. Chiu, Jan Kubelka, Regine Herbst-Irmer, William A. Eaton, James Hofrichter, and David R. Davies. "High-resolution x-ray crystal structures of the villin headpiece subdomain, an ultrafast folding protein". en. In: Proceedings of the National Academy of Sciences 102.21 (May 2005), pp. 7517-7522. ISSN: 0027-8424, 1091-6490. DOI: 10 . $1073 /$ pnas . 0502495102, URL: http: / / www . pnas . org/ content/ $102 / 21 / 7517$

[33] Simona Cocco, Remi Monasson, and Martin Weigt. "From Principal Component to Direct Coupling Analysis of Coevolution in Proteins: Low-Eigenvalue Modes are Needed for Structure Prediction". en. In: PLoS Computational Biology 9.8 (Aug. 2013). Ed. by Björn Wallner, e1003176. ISSN: 1553-7358. DOI: 10 . 1371 / journal . pcbi. 1003176, URL: http: / / dx.plos . org/10.1371/ journal. pcbi.1003176 (visited on 09/22/2015).

[34] Mark O. Collins, Lu Yu, Iain Campuzano, Seth G. N. Grant, and Jyoti S. Choudhary. "Phosphoproteomic Analysis of the Mouse Brain Cytosol Reveals a Predominance of Protein Phosphorylation in Regions of Intrinsic Sequence Disorder". en. In: Molecular \& Cellular Proteomics 7.7 (July 2008), pp. 1331-1348. ISSN: 1535-9476, 1535-9484. DOI: 10 . 
BIBLIOGRAPHY

1074/mcp . M700564-MCP200, URL: http: / / www . mcponline. org/content/7/7/1331.

[35] Susana Cristobal, Adam Zemla, Daniel Fischer, Leszek Rychlewski, and Arne Elofsson. "A study of quality measures for protein threading models". en. In: BMC Bioinformatics 2.1 (Dec. 2001), p. 5. ISSN: 1471-2105. DOI: $10.1186 / 1471-2105-2-5$. URL: https : / / link . springer.com/article/10.1186/1471-2105-2-5 (visited on 09/18/2017).

[36] Gavin E Crooks, Gary Hon, John-Marc Chandonia, and Steven E Brenner. "WebLogo: a sequence logo generator." In: Genome research 14.6 (June 2004), pp. 1188-90. DOI: 10 . 1101/gr. 849004 . URL: http : / / www . pubmedcentral . nih . gov / articlerender. fcgi ? artid $=419797 \&$ tool $=$ pmcentrez \& rendertype $=$ abstract.

[37] Charles Darwin. On the origin of species by means of natural selection, or the preservation of favoured races in the struggle for life. $5 \mathrm{th}$. London: Murray, 1869. URL: http: / / darwin-online.org.uk/ contents. html\#origin.

[38] Warren L. DeLano and Legion. The PyMOL Molecular Graphics System. 2018. URL: https: / / sourceforge.net/projects/pymol/ (visited on 04/13/2018).

[39] Cyril Dominguez, Rolf Boelens, and Alexandre M. J. J. Bonvin. "HADDOCK: A ProteinProtein Docking Approach Based on Biochemical or Biophysical Information". In: Journal of the American Chemical Society 125.7 (Feb. 2003), pp. 1731-1737. ISSN: 0002-7863. DOI: $10.1021 /$ ja026939x. URL: https: / / doi.org/10.1021/ ja026939x (visited on 03/16/2018).

[40] A. K. Dunker, E. Garner, S. Guilliot, P. Romero, K. Albrecht, J. Hart, Z. Obradovic, C. Kissinger, and J. E. Villafranca. "Protein disorder and the evolution of molecular recognition: theory, predictions and observations". eng. In: Pacific Symposium on Biocomputing. Pacific Symposium on Biocomputing (1998), pp. 473-484. ISSN: 2335-6928.

[41] A. Keith Dunker, J. David Lawson, Celeste J Brown, Ryan M Williams, Pedro Romero, Jeong S Oh, Christopher J Oldfield, Andrew M Campen, Catherine M Ratliff, Kerry W Hipps, Juan Ausio, Mark S Nissen, Raymond Reeves, ChulHee Kang, Charles R Kissinger, Robert W Bailey, Michael D Griswold, Wah Chiu, Ethan C Garner, and Zoran Obradovic. "Intrinsically disordered protein". In: Journal of Molecular Graphics and Modelling 19.1 (Feb. 2001), pp. 26-59. ISSN: 1093-3263. DOI: 10 .1016/S1093-3263 (00) 00138-8. URL: http://wWw. sciencedirect.com/science/article/pii/ S1093326300001388. 
[42] A. Keith Dunker, Pedro Romero, Zoran Obradovic, Ethan C. Garner, and Celeste J. Brown. "Intrinsic Protein Disorder in Complete Genomes". en. In: Genome Informatics 11 (2000), pp. 161-171. ISSN: 0919-9454, 2185-842X. DOI: 10 . 11234 / gi1990 . 11 . 161. URL: https://www.jstage.jst.go.jp/article/gi1990/11/ 0/11_0_161/_article/-char/ja/

[43] A Keith Dunker, Israel Silman, Vladimir N Uversky, and Joel L Sussman. "Function and structure of inherently disordered proteins". In: Current Opinion in Structural Biology. Catalysis and regulation / Proteins 18.6 (Dec. 2008), pp. 756-764. ISSN: 0959-440X. DOI:10.1016/ j.sbi.2008.10.002, URL: http://www.sciencedirect.com/ science/article/pii/s0959440X08001516.

[44] S D Dunn, L M Wahl, and G B Gloor. "Mutual information without the influence of phylogeny or entropy dramatically improves residue contact prediction." In: Bioinformatics (Oxford, England) 24.3 (Feb. 2008), pp. 333-40. DOI: 10.1093/bioinformatics/btm604. URL: http://www.ncbi.nlm.nih.gov/pubmed/18057019.

[45] H. Jane Dyson and Peter E. Wright. "Intrinsically unstructured proteins and their functions". en. In: Nature Reviews Molecular Cell Biology 6.3 (Mar. 2005), pp. 197-208. ISSN: 1471-0080. DOI: 10 . $1038 /$ nrm1589. URL: https : / / www - nature . com / articles / nrm1589

[46] Sean R. Eddy. "Accelerated Profile HMM Searches". en. In: PLoS Computational Biology 7.10 (Oct. 2011). Ed. by William R. Pearson, e1002195. ISSN: 1553-7358. DOI: 10 . $1371 /$ journal . pcbi. 1002195 URL: http: / / dx.plos . org/10 .1371/ journal. pcbi.1002195.

[47] Magnus Ekeberg, Cecilia Lövkvist, Yueheng Lan, Martin Weigt, and Erik Aurell. "Improved contact prediction in proteins: Using pseudolikelihoods to infer Potts models". In: Physical Review E 87.1 (Jan. 2013), pp. 012707-012707. DOI: 10 .1103/PhysRevE . 87 . 012707. URL: http:// link.aps .org/doi/10.1103/PhysRevE . 87 . 012707

[48] Arne Elofsson, Keehyoung Joo, Chen Keasar, Jooyoung Lee, Ali H. A. Maghrabi, Balachandran Manavalan, Liam J. McGuffin, David Ménendez Hurtado, Claudio Mirabello, Robert Pilstål, Tomer Sidi, Karolis Uziela, and Björn Wallner. "Methods for estimation of model accuracy in CASP12". eng. In: Proteins (Oct. 2017). ISSN: 1097-0134. DOI: $10.1002 /$ prot .25395 
[49] S. Walter Englander and Leland Mayne. "The nature of protein folding pathways". en. In: Proceedings of the National Academy of Sciences 111.45 (Nov. 2014), pp. 15873-15880. ISSN: 0027-8424, 1091-6490. DOI: 10.1073/pnas.1411798111, URL: http: / /www . pnas . org/ content/111/45/15873 (visited on 03/27/2018).

[50] Leonhard Euler. "Solutio problematis ad geometriam situs pertinentis". In: Comment. Acad. Sci. U. Petrop 8 (1736), pp. 128-40.

[51] Embaie Ferrow and Madis Roots. "A preparation technique for TEM specimens; application to synthetic Mg-chlorite". en. In: European Journal of Mineralogy (Dec. 1989), pp. 815-820. ISSN: , DOI: $10.1127 /$ ejm/1/6/0815, URL: https : / / www. schweizerbart. de/ papers/ejm/detail/1/82531/A_preparation_technique_. for_TEM_specimens_applicat?af=crossref

[52] Matteo Figliuzzi, Hervé Jacquier, Alexander Schug, Oliver Tenaillon, and Martin Weigt. "Coevolutionary Landscape Inference and the Context-Dependence of Mutations in Beta-Lactamase TEM-1". en. In: Molecular Biology and Evolution 33.1 (Jan. 2016), pp. 268-280. ISSN: 0737-4038. DOI: 10 . $1093 / \mathrm{molbev} / \mathrm{msv} 211$. URL: https : //academic.oup.com/mbe/article/33/1/268/2579532.

[53] Robert D. Finn, Penelope Coggill, Ruth Y. Eberhardt, Sean R. Eddy, Jaina Mistry, Alex L. Mitchell, Simon C. Potter, Marco Punta, Matloob Qureshi, Amaia Sangrador-Vegas, Gustavo A. Salazar, John Tate, and Alex Bateman. "The Pfam protein families database: towards a more sustainable future". en. In: Nucleic Acids Research 44.D1 (Jan. 2016), pp. D279-D285. ISSN: 0305-1048, 1362-4962. DOI: 10 . $1093 /$ nar $/$ gkv1344. URL: http: // nar.oxfordjournals.org/lookup/ doi/10.1093/nar/gkv1344.

[54] Robert D. Finn, Jaina Mistry, Benjamin Schuster-Böckler, Sam Griffiths-Jones, Volker Hollich, Timo Lassmann, Simon Moxon, Mhairi Marshall, Ajay Khanna, Richard Durbin, Sean R. Eddy, Erik L. L. Sonnhammer, and Alex Bateman. "Pfam: clans, web tools and services". en. In: Nucleic Acids Research 34.suppl_1 (Jan. 2006), pp. D247-D251. ISSN: 0305-1048. DOI: 10.1093/nar/gk j149. URL: https://academic.oup.com/nar/article/34/suppl_1/ D247/1133922.

[55] James S. Fraser, Michael W. Clarkson, Sheena C. Degnan, Renske Erion, Dorothee Kern, and Tom Alber. "Hidden alternative structures of proline isomerase essential for catalysis". en. In: Nature 462.7273 (Dec. 2009), pp. 669-673. ISSN: 1476-4687. DOI: 10 . $1038 /$ nature08615. URL: https : / / www . nature.com/articles / nature08615 
Bibliography

[56] H. Frauenfelder, S. G. Sligar, and P. G. Wolynes. "The energy landscapes and motions of proteins". en. In: Science 254.5038 (Dec. 1991), pp. 1598-1603. ISSN: 0036-8075, 1095-9203. DOI: $10.1126 /$ science. 1749933. URL: http://science.sciencemag.org/content/ $254 / 5038 / 1598$.

[57] Jerome Friedman, Trevor Hastie, and Robert Tibshirani. "Sparse inverse covariance estimation with the graphical lasso." In: Biostatistics (Oxford, England) 9.3 (July 2008), pp. 432-41. DOI: $10.1093 /$ biostatistics / kxm045, URL: http: / / www . pubmedcentral. nih.gov/articlerender. fcgi ? artid $=3019769 \&$ tool $=$ pmcentrez\&rendertype=abstract.

[58] Monika Fuxreiter. "Fuzziness: linking regulation to protein dynamics". en. In: Mol. BioSyst. 8.1 (2012), pp. 168-177. ISSN: 1742-206X, 17422051. DOI: 10 . 1039 / C1MB05234A URL: http: / / xlink . rSC . org $/$ ?DOI=C1MB05234A

[59] Robert J. C. Gilbert, Paola Fucini, Sean Connell, Stephen D. Fuller, Knud H. Nierhaus, Carol V. Robinson, Christopher M. Dobson, and David I. Stuart. "Three-Dimensional Structures of Translating Ribosomes by Cryo-EM". In: Molecular Cell 14.1 (Apr. 2004), pp. 57-66. ISSN: 1097-2765. DOI: $10.1016 /$ S1097-2765 (04) 00163-7. URL: http://www.sciencedirect.com/science/article/pii/ S1097276504001637.

[60] Krzysztof Ginalski, Arne Elofsson, Daniel Fischer, and Leszek Rychlewski. "3D-Jury: a simple approach to improve protein structure predictions". In: Bioinformatics 19.8 (May 2003), pp. 1015-1018. ISSN: 1367-4803. DOI: 10 . 1093 / bioinformatics / btg124 URL: https://academic.oup.com/bioinformatics/article/ 19/8/1015/235399/3D-Jury-a-simple-approach-toimprove-protein (visited on 09/08/2017).

[61] Moshe Goldsmith and Dan S Tawfik. "Enzyme engineering: reaching the maximal catalytic efficiency peak". In: Current Opinion in Structural Biology. Proteinnucleic acid interactions Catalysis and regulation 47 (Dec. 2017), pp. 140-150. ISSN: 0959-440X. DOI: $10.1016 / j$. sbi.2017.09.002. URL: http://www.sciencedirect.com/ science/article/pii/s0959440X17300647.

[62] Najeeb Halabi, Olivier Rivoire, Stanislas Leibler, and Rama Ranganathan. "Protein Sectors: Evolutionary Units of Three-Dimensional Structure". In: Cell 138.4 (Aug. 2009), pp. 774-786. ISSN: 00928674. DOI: $10.1016 /$ j . cell . 2009.07 .038 uRL: http : // / ww . sciencedirect . com / science/ article/pii/ S0092867409009635 (visited on 09/08/2017). 
[63] Jing-Dong J. Han, Nicolas Bertin, Tong Hao, Debra S. Goldberg, Gabriel F. Berriz, Lan V. Zhang, Denis Dupuy, Albertha J. M. Walhout, Michael E. Cusick, Frederick P. Roth, and Marc Vidal. "Evidence for dynamically organized modularity in the yeast proteinprotein interaction network". en. In: Nature 430.6995 (July 2004), pp. 88-93. ISSN: 1476-4687. DOI: 10 . 1038 / nature02555, URL: https : / / www . nature.com/articles/nature02555

[64] Paul M. Harrison and Mark Gerstein. "Studying Genomes Through the Aeons: Protein Families, Pseudogenes and Proteome Evolution". In: Journal of Molecular Biology 318.5 (May 2002), pp. 1155-1174. ISSN: 0022-2836. DOI: 10 . 1016/S0022-2836(02) 00109-2. URL: http://www.sciencedirect.com/science/article/pii/ S0022283602001092.

[65] Steven Hayward. "Structural principles governing domain motions in proteins". en. In: Proteins: Structure, Function, and Bioinformatics 36.4 (Sept. 1999), pp. 425-435. ISSN: 1097-0134. DOI: 10.1002 / (SICI) 1097-0134(19990901) 36:4<425::AID-PROT6>3.0.CO; 2-S. URL: https://onlinelibrary .wiley.com/doi/abs/ $10.1002 / \% 28$ SICI $\div 291097-0134 \div 2819990901 \% 2936 \div 3 A 4 \%$ $3 \mathrm{C} 425 \div 3 \mathrm{~A} \div 3 \mathrm{AAID}-\mathrm{PROT} 6 \div 3 \mathrm{E} 3.0 . \mathrm{CO} \div 3 \mathrm{~B} 2-\mathrm{S}$.

[66] Sara Helander, Meri Montecchio, Robert Pilstål, Yulong Su, Jacob Kuruvilla, Malin Elvén, Javed M.E. Ziauddin, Madhanagopal Anandapadamanaban, Susana Cristobal, Patrik Lundström, Rosalie C. Sears, Björn Wallner, and Maria Sunnerhagen. "Pre-Anchoring of Pin1 to Unphosphorylated c-Myc in a Fuzzy Complex Regulates cMyc Activity". en. In: Structure 23.12 (Dec. 2015), pp. 2267-2279. ISSN: 09692126. DOI: $10.1016 / j$. str. 2015 . 10 . 010 URL: http : / / linkinghub. elsevier. com / retrieve / pii / S0969212615004499 (visited on 02/01/2016).

[67] Thomas A. Hopf, Lucy J. Colwell, Robert Sheridan, Burkhard Rost, Chris Sander, and Debora S. Marks. "Three-Dimensional Structures of Membrane Proteins from Genomic Sequencing". English. In: Cell 149.7 (June 2012), pp. 1607-1621. ISSN: 0092-8674, 1097-4172. DOI: $10.1016 / j \cdot$ cell.2012.04.012 URL: http: / / www . cell. com/ cell / abstract/S0092-8674 (12)00509-0 (visited on 09/05/2017).

[68] William Humphrey, Andrew Dalke, and Klaus Schulten. "VMD Visual Molecular Dynamics". In: Journal of Molecular Graphics 14 (1996). bibtex: HUMP96 bibtex[tbstatus=Published.;techrep=;tbreference=222], pp. 33-38. 
Bibliography

[69] Shah Jalal, Gunnar Wretlind, Naomasa Gotoh, and Bengt Wretlind. "Rapid identification of mutations in a multidrug efflux pump in Pseudomonas aeruginosa". en. In: APMIS 107.7-12 (Mar. 1999), pp. 1109-1116. ISSN: 1600-0463. DOI: $10.1111 / j .1699-0463$. 1999.tb01516.x. URL:https://onlinelibrary.wiley.com/ doi/abs/10.1111/j.1699-0463.1999.tb01516.x

[70] David T Jones, Daniel W a Buchan, Domenico Cozzetto, and Massimiliano Pontil. "PSICOV: precise structural contact prediction using sparse inverse covariance estimation on large multiple sequence alignments." In: Bioinformatics (Oxford, England) 28.2 (Jan. 2012), pp. 184-90. DOI:10.1093/bioinformatics/btr638. URL: http: //www.ncbi.nlm.nih.gov/pubmed/22101153

[71] W. Kabsch. "A solution for the best rotation to relate two sets of vectors". en. In: Acta Crystallographica Section A: Crystal Physics, Diffraction, Theoretical and General Crystallography 32.5 (Sept. 1976), pp. 922923. ISSN: 0567-7394. DOI: 10 . 1107 / S0567739476001873, URL: // scripts.iucr.org/cgi-bin/paper?a12999 (visited on 03/28/2018).

[72] J. C. Kendrew, G. Bodo, H. M. Dintzis, R. G. Parrish, H. Wyckoff, and D. C. Phillips. "A Three-Dimensional Model of the Myoglobin Molecule Obtained by X-Ray Analysis". en. In: Nature 181.4610 (Mar. 1958), pp. 662-666. ISSN: 1476-4687. DOI: $10.1038 / 181662$ a 0. URL: https://www.nature.com/articles/181662a0

[73] George A. Khoury, Richard C. Baliban, and Christodoulos A. Floudas. "Proteome-wide post-translational modification statistics: frequency analysis and curation of the swiss-prot database". en. In: Scientific Reports 1 (Sept. 2011), p. 90. ISSN: 2045-2322. DOI: 10 . $1038 /$ srep00090, URL: https : / / www . nature.com/articles / srep00090

[74] Arthur Koestler. The ghost in the machine. eng. OCLC: 20932999. London: Arkana, 1989. ISBN: 978-0-14-019192-9.

[75] D. E. Koshland, G. Némethy, and D. Filmer. "Comparison of Experimental Binding Data and Theoretical Models in Proteins Containing Subunits*". In: Biochemistry 5.1 (Jan. 1966), pp. 365-385. ISSN: 00062960. DOI:10.1021/bi00865a047. URL:https://doi.org/10. 1021/bi00865a047

[76] Günter Kramer, Daniel Boehringer, Nenad Ban, and Bernd Bukau. "The ribosome as a platform for co-translational processing, folding and targeting of newly synthesized proteins". en. In: Nature Structural E Molecular Biology 16.6 (June 2009), pp. 589-597. ISSN: 1545-9985. DOI: $10.1038 / \mathrm{nsmb} .1614$. URL: https: / / www . nature.com/ articles/nsmb.1614 
[77] Werner G. Krebs and Mark Gerstein. "SURVEY AND SUMMARYThe morph server: a standardized system for analyzing and visualizing macromolecular motions in a database framework". en. In: Nucleic Acids Research 28.8 (Apr. 2000), pp. 1665-1675. ISSN: 0305-1048. DOI: 10.1093/nar/28.8.1665. URL: https://academic.oup.com/ nar/article/28/8/1665/1009208

[78] Christopher Langan. "An Introduction to Mathematical Metaphysics". en. In: Cosmos and History: The Journal of Natural and Social Philosophy 13.2 (Mar. 2017), pp. 313-330. ISSN: 1832-9101. URL: https : / / cosmosandhistory . org/index . php/ journal/ article/view/618

[79] Robin van der Lee, Marija Buljan, Benjamin Lang, Robert J. Weatheritt, Gary W. Daughdrill, A. Keith Dunker, Monika Fuxreiter, Julian Gough, Joerg Gsponer, David T. Jones, Philip M. Kim, Richard W. Kriwacki, Christopher J. Oldfield, Rohit V. Pappu, Peter Tompa, Vladimir N. Uversky, Peter E. Wright, and M. Madan Babu. "Classification of Intrinsically Disordered Regions and Proteins". In: Chemical Reviews 114.13 (July 2014), pp. 6589-6631. ISSN: 0009-2665. DOI: 10 . 1021 / cr400525m. URL: https : / / doi . org/10 . 1021/ cr $400525 \mathrm{~m}$

[80] Michael Levitt and Cyrus Chothia. "Structural patterns in globular proteins". en. In: Nature 261.5561 (June 1976), pp. 552-558. ISSN: 00280836. DOI: 10 . 1038 / 261552a0, URL: http : / / www . nature. com/nature/journal/v261/n5561/abs/261552a0.html? foxtrotcallback=true.

[81] Michael Levitt and Mark Gerstein. "A unified statistical framework for sequence comparison and structure comparison". en. In: Proceedings of the National Academy of Sciences 95.11 (May 1998), pp. 59135920. ISSN: 0027-8424, 1091-6490. URL: http: / / www • pnas . org/ content/95/11/5913 (visited on 09/18/2017).

[82] Yeong-Shin Lin, Wei-Lun Hsu, Jenn-Kang Hwang, and Wen-Hsiung Li. "Proportion of Solvent-Exposed Amino Acids in a Protein and Rate of Protein Evolution". en. In: Molecular Biology and Evolution 24.4 (Apr. 2007), pp. 1005-1011. ISSN: 0737-4038. DOI: $10.1093 / \mathrm{mol} \mathrm{bev} /$ msm019. URL:https://academic.oup.com/mbe/article/24/ 4/1005/1012960.

[83] Yih-Cherng Liou, Xiao Zhen Zhou, and Kun Ping Lu. "Prolyl isomerase Pin1 as a molecular switch to determine the fate of phosphoproteins". In: Trends in Biochemical Sciences 36.10 (Oct. 2011), pp. 501514. ISSN: 0968-0004. DOI: $10.1016 / j$.tibs.2011.07.001. URL: http://www.sciencedirect.com/science/article/pii/ S0968000411001125. 
Bibliography

[84] Kun Ping Lu and Xiao Zhen Zhou. "The prolyl isomerase PIN1: a pivotal new twist in phosphorylation signalling and disease." In: $\mathrm{Na}$ ture reviews. Molecular cell biology 8.11 (Nov. 2007), pp. 904-16. DOI: 10.1038/nrm2261. URL: http: //www.ncbi.nlm.nih.gov/ pubmed/17878917

[85] Ulrike Luxburg. "A tutorial on spectral clustering". In: Statistics and Computing 17.4 (Aug. 2007), pp. 395-416. DOI: 10 . 1007/s11222007-9033-z, URL: http:// link. springer.com/10.1007/ s11222-007-9033-z.

[86] Edward Lyman and Daniel M. Zuckerman. "Ensemble-Based Convergence Analysis of Biomolecular Trajectories". In: Biophysical Journal 91.1 (July 2006), pp. 164-172. ISSN: 0006-3495. DOI: 10 . 1529/ biophysj.106.082941. URL: http: / / www . sciencedirect . com/science/article/pii/s0006349506717167 (visited on 04/06/2018).

[87] Valerio Mariani, Marco Biasini, Alessandro Barbato, and Torsten Schwede. "IDDT: a local superposition-free score for comparing protein structures and models using distance difference tests". In: Bioinformatics 29.21 (Nov. 2013), pp. 2722-2728. ISSN: 1367-4803. DOI: 10 . 1093 / bioinformatics/btt473, URL: https : / / academic . oup.com/bioinformatics/article/29/21/2722/195896/ 1DDT-a-local-superposition-free-score-for (visited on 09/18/2017).

[88] L C Martin, G B Gloor, S D Dunn, and L M Wahl. "Using information theory to search for co-evolving residues in proteins." In: Bioinformatics (Oxford, England) 21.22 (Nov. 2005), pp. 4116-24. DOI: $10.1093 /$ bioinformatics/bti671. URL: http://www.ncbi.nlm.nih. gov/pubmed/16159918

[89] Joanna Masel. "Genetic drift". In: Current Biology 21.20 (Oct. 2011), R837-R838. ISSN: 0960-9822. DOI: $10.1016 / j . c u b .2011 .08 .007$. URL: http://www.sciencedirect.com/science/article/ pii/S0960982211008827

[90] Nicolai Meinshausen and Peter Bühlmann. "High-dimensional graphs and variable selection with the Lasso". In: The Annals of Statistics 34.3 (June 2006), pp. 1436-1462. DOI: 10 . $1214 /$ 009053606000000281, URL: http: / / projecteuclid.org/ Dienst/getRecord?id=euclid.aos/1152540754/

[91] Natalie Meyer and Linda Z Penn. "Reflecting on 25 years with MYC". In: Nature Reviews Cancer 8.December (2008), pp. 976-990. 
[92] Jacque Monod, Jeffries Wyman, and Jean-Pierre Changeux. "On the nature of allosteric transitions: A plausible model". In: Journal of Molecular Biology 12.1 (May 1965), pp. 88-118. ISSN: 00222836. DOI: 10 . $1016 /$ S0022-2836(65) 80285-6. URL: http : // / ww . sciencedirect. com / science/article/pii/ S0022283665802856

[93] Faruck Morcos, Andrea Pagnani, Bryan Lunt, Arianna Bertolino, Debora S Marks, Chris Sander, Riccardo Zecchina, José N Onuchic, Terence Hwa, and Martin Weigt. "Direct-coupling analysis of residue coevolution captures native contacts across many protein families." In: Proceedings of the National Academy of Sciences of the United States of America 108.49 (Dec. 2011), E1293-301. DOI: 10 . 1073 / pnas . 1111471108, URL: http: / / www • pubmedcentral . nih.gov/ articlerender. fcgi ? artid $=3241805 \&$ tool = pmcentrez $\&$ rendertype=abstract

[94] Sara Mostafavi, Debajyoti Ray, David Warde-Farley, Chris Grouios, and Quaid Morris. "GeneMANIA: a real-time multiple association network integration algorithm for predicting gene function". In: Genome Biology 9.Suppl 1 (2008), S4. ISSN: 1465-6906. DOI:10.1186/ gb-2008-9-s1-s4. URL: https: //wWw.ncbi.nlm.nih.gov/ pmc/articles/PMC2447538/(visited on 04/06/2018).

[95] Hesam N Motlagh, James O Wrabl, Jing Li, and Vincent J Hilser. "The ensemble nature of allostery." In: Nature 508.7496 (Apr. 2014), pp. 3319. DOI: $10.1038 /$ nature13001. URL: http://www. ncbi.nlm. nih.gov/pubmed/24740064.

[96] Alexey G. Murzin, Steven E. Brenner, Tim Hubbard, and Cyrus Chothia. "SCOP: A structural classification of proteins database for the investigation of sequences and structures". In: Journal of Molecular Biology 247.4 (Apr. 1995), pp. 536-540. ISSN: 0022-2836. DOI: 10 . 1016/S0022 - 2836(05 ) 80134-2. URL: http : // / ww . sciencedirect. com/ science/article/pii / S0022283605801342

[97] Alfred Nobel. The Nobel Prize in Chemistry 2009. 2009. URL: https: // www - nobelprize . org / nobel_prizes / chemistry / laureates/2009/(visited on 04/13/2018).

[98] Alfred Nobel. The Nobel Prize in Physiology or Medicine 1974. 1974. URL: https://www . nobelprize.org/nobel_prizes/medicine/ laureates/1974/(visited on 04/13/2018).

[99] Christopher J. Oldfield and A. Keith Dunker. "Intrinsically Disordered Proteins and Intrinsically Disordered Protein Regions". In: Annual Review of Biochemistry 83.1 (2014), pp. 553-584. DOI: 10 . 1146/ 
annurev-biochem-072711-164947, URL: https: / / doi.org/ 10.1146/annurev-biochem-072711-164947.

[100] Kliment Olechnovi, Eleonora Kulberkyt, and eslovas Venclovas. "CAD-score: A new contact area difference-based function for evaluation of protein structural models". en. In: Proteins: Structure, Function, and Bioinformatics 81.1 (Jan. 2013), pp. 149-162. ISSN: 1097-0134. DOI: 10 . 1002 /prot . 24172. URL: http: / / onlinelibrary .wiley. com/doi/10.1002/prot.24172/abstract.

[101] C a Orengo, a D Michie, S Jones, D T Jones, M B Swindells, and J M Thornton. "CATH-a hierarchic classification of protein domain structures." In: Structure (London, England : 1993) 5.8 (Aug. 1997), pp. 1093108. URL: http://www.ncbi.nlm.nih.gov/pubmed/9309224.

[102] Christine A. Orengo, David T. Jones, and Janet M. Thornton. "Protein superfamilles and domain superfolds". en. In: Nature 372.6507 (Dec. 1994), pp. 631-634. ISSN: 0028-0836. DOI: 10 . 1038 / 372631 a 0. URL: http: / / www . nature.com/nature/journal/v372/n6507/ abs/372631a0.html?foxtrotcallback=true

[103] Eric A. Ortlund, Jamie T. Bridgham, Matthew R. Redinbo, and Joseph W. Thornton. "Crystal structure of an ancient protein". In: Science (New York, N.Y.) 317.5844 (Sept. 2007), pp. 1544-1548. ISSN: 0036-8075. DOI: 10 .1126/science.1142819. URL: https : / / wWw . ncbi. nlm.nih.gov/pmc/articles/PMC2519897/

[104] George E. Palade. "A Small Particulate Component of the Cytoplasm". en. In: The Journal of Cell Biology 1.1 (Jan. 1955), pp. 59-68. ISSN: 0021-9525, 1540-8140. DOI: 10 . 1083 / jcb.1.1.59. URL: http://jcb.rupress.org/content/1/1/59.

[105] Szilárd Páll, Mark James Abraham, Carsten Kutzner, Berk Hess, and Erik Lindahl. "Tackling Exascale Software Challenges in Molecular Dynamics Simulations with GROMACS". en. In: Solving Software Challenges for Exascale. Lecture Notes in Computer Science. Springer, Cham, Apr. 2014, pp. 3-27. ISBN: 978-3-319-15975-1 978-3-319-15976-8. DOI: 10 . 1007/978-3-319-15976-8_1. URL: https : / / link . springer.com/chapter/10.1007/978-3-319-15976-8_1.

[106] David Penny. "Epigenetics, Darwin, and Lamarck". In: Genome Biology and Evolution 7.6 (May 2015), pp. 1758-1760. ISSN: 1759-6653. DOI: 10 . 1093 / gbe / evv107. URL: https: / / www . ncbi.nlm.nih. gov/pmc/articles/PMC4494054/

[107] Inoka C. Perera and Anne Grove. "Molecular Mechanisms of LigandMediated Attenuation of DNA Binding by MarR Family Transcriptional Regulators". en. In: Journal of Molecular Cell Biology 2.5 (Oct. 2010), pp. 243-254. ISSN: 1674-2788. DOI: 10 . 1093 / jmcb/m jq021. 
URL: https://academic.oup.com/jmcb/article/2/5/243/ 868608

[108] Robert Pilstål, Maria Sunnerhagen, and Björn Wallner. "Functional Interaction Network of c-MYC Conserved Regions determined by Evolutionary Couplings". Apr. 2018.

[109] Robert Pilstål and Björn Wallner. "Improvements in Protein Model Quality Assessment from Automated Domain Partitioning using Spectral Clustering". Apr. 2018.

[110] Sander Pronk, Szilárd Páll, Roland Schulz, Per Larsson, Pär Bjelkmar, Rossen Apostolov, Michael R Shirts, Jeremy C Smith, Peter M Kasson, David van der Spoel, Berk Hess, and Erik Lindahl. "GROMACS 4.5: a high-throughput and highly parallel open source molecular simulation toolkit." In: Bioinformatics (Oxford, England) 29.7 (Feb. 2013), pp. 845-854. DOI:10.1093/bioinformatics/btt055 URL: http://www.ncbi.nlm.nih.gov/pubmed/23407358

[111] Barak Raveh, Nir London, Lior Zimmerman, and Ora SchuelerFurman. "Rosetta FlexPepDock ab-initio: simultaneous folding, docking and refinement of peptides onto their receptors." In: PloS one 6.4 (Jan. 2011), e18934-e18934. DOI: 10 . 1371 / journal • pone. 0018934, URL: http: / / www - pubmedcentral . nih.gov / articlerender. fcgi ? artid $=3084719 \&$ tool = pmcentrez \& rendertype $=$ abstract

[112] Kimberly A. Reynolds, Richard N. McLaughlin, and Rama Ranganathan. "Hot Spots for Allosteric Regulation on Protein Surfaces". In: Cell 147.7 (Dec. 2011), pp. 1564-1575. ISSN: 0092-8674. DOI: $10 \cdot 1016 / j \cdot \mathrm{cell} \cdot 2011 \cdot 10 \cdot 049$. URL: http : // www . sciencedirect. com / science/article/pii/ S0092867411013626.

[113] Liat Rockah-Shmuel, Ágnes Tóth-Petróczy, and Dan S Tawfik. "Systematic Mapping of Protein Mutational Space by Prolonged Drift Reveals the Deleterious Effects of Seemingly Neutral Mutations." In: PLoS computational biology 11.8 (Aug. 2015), e1004421-e1004421. DOI: 10.1371/journal.pcbi.1004421. URL: http: //www.ncbi. nlm.nih.gov/pubmed/26274323

[114] B. Rost, J. Liu, R. Nair, K. O. Wrzeszczynski, and Y. Ofran. "Automatic prediction of protein function". en. In: Cellular and Molecular Life Sciences CMLS 60.12 (Dec. 2003), pp. 2637-2650. ISSN: 1420-682X, 1420-9071. DOI: 10 . 1007 / s00018-003-3114-8, URL: https : //link.springer.com/article/10.1007/s00018-003$3114-8$ 
[115] Avner Schlessinger, Christian Schaefer, Esmeralda Vicedo, Markus Schmidberger, Marco Punta, and Burkhard Rost. "Protein disordera breakthrough invention of evolution?" In: Current Opinion in Structural Biology 21.3 (June 2011), pp. 412-418. ISSN: 0959-440X. DOI: $10 \cdot 1016 / j \cdot$ sbi . 2011 . 03 . 014 URL: http : / / www . sciencedirect . com / science / article / pii / S0959440X11000662

[116] Frank Schluenzen, Ante Tocilj, Raz Zarivach, Joerg Harms, Marco Gluehmann, Daniela Janell, Anat Bashan, Heike Bartels, Ilana Agmon, François Franceschi, and Ada Yonath. "Structure of Functionally Activated Small Ribosomal Subunit at $3.3 \AA$ A Resolution". English. In: Cell 102.5 (Sept. 2000), pp. 615-623. ISSN: 0092-8674, 1097-4172. DOI: 10.1016/S0092-8674(00)00084-2. URL: http://wWw. cell. com/cell/abstract/S0092-8674(00)00084-2.

[117] Frank H Schumann, Hubert Riepl, Till Maurer, Wolfram Gronwald, Klaus-Peter Neidig, and Hans Robert Kalbitzer. "Combined chemical shift changes and amino acid specific chemical shift mapping of protein-protein interactions." In: Journal of biomolecular NMR 39.4 (Dec. 2007), pp. 275-89. DOI: $10.1007 /$ s10858-007-9197-z. URL: http://www.ncbi.nlm.nih.gov/pubmed/17955183

[118] Scotland Robert W. "Deep homology: A view from systematics". In: BioEssays 32.5 (Apr. 2010), pp. 438-449. ISSN: 0265-9247. DOI: 10 . 1002 / bies . 200900175, URL: https : / / onlinelibrary . wiley.com/doi/abs/10.1002/bies.200900175

[119] Michele Seeber, Angelo Felline, Francesco Raimondi, Stefanie Muff, Ran Friedman, Francesco Rao, Amedeo Caflisch, and Francesca Fanelli. "Wordom: A user-friendly program for the analysis of molecular structures, trajectories, and free energy surfaces". en. In: Journal of Computational Chemistry 32.6 (Apr. 2011), pp. 1183-1194. ISSN: 01928651. DOI: $10.1002 /$ jcc.21688, URL: http://doi.wiley. $\mathrm{com} / 10.1002 / \mathrm{jcc} .21688$

[120] C. E. Shannon. "A Mathematical Theory of Communication". In: The Bell System Technical Journal 27 (1948), pp. 379-423.

[121] J Shi and J Malik. "Normalized Cuts and Image Segmentation". In: Ieee Transactions on Pattern Analysis and Machine Intelligence 22.8 (2000), pp. 888-905. ISSN: 0818678224. DOI: $10.1109 / 34.868688$. URL: http://www . computer.org/portal/web/csdl/doi ? doc $=$ abs/proceedings/cvpr/1997/7822/00/78220731abs.htmo 5Cnpapers3: / / publication/uuid/268FC197-AF47-4C7C887F-BEDB94A81320. 
[122] Rob Shields. "Cultural Topology: The Seven Bridges of Königsburg, 1736". en. In: Theory, Culture \& Society 29.4-5 (July 2012), pp. 43-57. ISSN: 0263-2764. DOI: $10.1177 / 0263276412451161$, URL: https: //doi.org/10.1177/0263276412451161.

[123] D Shortle, K T Simons, and D Baker. "Clustering of low-energy conformations near the native structures of small proteins." In: Proceedings of the National Academy of Sciences of the United States of America 95.19 (Sept. 1998), pp. 11158-62. URL: http : / / www . pubmedcentral . nih.gov/articlerender. fcgi ? artid $=$ 21612\&tool=pmcentrez\&rendertype=abstract

[124] Michael K. Skinner. "Environmental Epigenetics and a Unified Theory of the Molecular Aspects of Evolution: A Neo-Lamarckian Concept that Facilitates Neo-Darwinian Evolution". In: Genome Biology and Evolution 7.5 (Apr. 2015), pp. 1296-1302. ISSN: 1759-6653. DOI: 10.1093/gbe/evv073, URL: https: //www.ncbi.nlm.nih. gov/pmc/articles/PMC4453068/

[125] Vladislav Soroka, Kateryna Kolkova, Jette S Kastrup, Kay Diederichs, Jason Breed, Vladislav V Kiselyov, Flemming M Poulsen, Ingrid K Larsen, Wolfram Welte, Vladimir Berezin, Elisabeth Bock, and Christina Kasper. "Structure and Interactions of NCAM Ig1-2-3 Suggest a Novel Zipper Mechanism for Homophilic Adhesion". In: Structure 11.10 (Oct. 2003), pp. 1291-1301. ISSN: 0969-2126. DOI: $10.1016 /$ j.str.2003.09.006. URL: http://www.sciencedirect.com/ science/article/pii/S0969212603002077.

[126] G. Spencer Brown. Laws of form. OCLC: 246480679. New York: Dutton, 1979. ISBN: 978-0-525-47544-6.

[127] Tyler N. Starr and Joseph W. Thornton. "Epistasis in protein evolution". en. In: Protein Science 25.7 (July 2016), pp. 1204-1218. ISSN: 1469896X. DOI: $10.1002 /$ pro.2897 URL: https://onlinelibrary. wiley.com/doi/abs/10.1002/pro.2897.

[128] Olga Khersonsky and Dan S. Tawfik. "Enzyme Promiscuity: A Mechanistic and Evolutionary Perspective". In: Annual Review of Biochemistry 79.1 (June 2010), pp. 471-505. ISSN: 0066-4154. DOI: 10 . 1146/ annurev-biochem-030409-143718, URL: https : / / ww . annualreviews . org/doi/10.1146/annurev-biochem030409-143718.

[129] Nobuhiko Tokuriki and Dan S. Tawfik. "Protein Dynamism and Evolvability". en. In: Science 324.5924 (Apr. 2009), pp. 203-207. ISSN: 0036-8075, 1095-9203. DOI: 10 . 1126 / science . 1169375. URL: http://science.sciencemag.org/content/324/5924/203 
Bibliography

[130] Cristian Tomasetti, Lu Li, and Bert Vogelstein. "Stem cell divisions, somatic mutations, cancer etiology, and cancer prevention". en. In: Science 355.6331 (Mar. 2017), pp. 1330-1334. ISSN: 0036-8075, 10959203. DOI:10.1126/science.aaf9011. URL: http://science. sciencemag.org/content/355/6331/1330.

[131] Peter Tompa and Monika Fuxreiter. "Fuzzy complexes: polymorphism and structural disorder in proteinprotein interactions". In: Trends in Biochemical Sciences 33.1 (Jan. 2008), pp. 2-8. ISSN: 09680004. DOI: 10 . $1016 / j$. tibs . 2007 . 10 . 003 uRL: http : // Www . sciencedirect . com / science / article / pii / S096800040700285X.

[132] Agnes Toth-Petroczy, Perry Palmedo, John Ingraham, Thomas A. Hopf, Bonnie Berger, Chris Sander, and Debora S. Marks. "Structured States of Disordered Proteins from Genomic Sequences". en. In: Cell 167.1 (Sept. 2016), 158-170.e12. ISSN: 00928674. DOI: $10.1016 / j$. cell.2016.09.010, URL: http: / / linkinghub.elsevier. com/retrieve/pii/s0092867416312430.

[133] Farida Tripodi, Raffaele Nicastro, Veronica Reghellin, and Paola Coccetti. "Post-translational modifications on yeast carbon metabolism: Regulatory mechanisms beyond transcriptional control". In: Biochimica et Biophysica Acta (BBA) - General Subjects 1850.4 (Apr. 2015), pp. 620-627. ISSN: 0304-4165. DOI: 10 . $1016 /$ j . bbagen . 2014 . 12.010 URL: http: / / wWw . sciencedirect. com/science/ article/pii/s0304416514004103.

[134] William B. Tu, Sara Helander, Robert Pilstål, K. Ashley Hickman, Corey Lourenco, Igor Jurisica, Brian Raught, Björn Wallner, Maria Sunnerhagen, and Linda Z. Penn. "Myc and its interactors take shape". en. In: Biochimica et Biophysica Acta (BBA) - Gene Regulatory Mechanisms 1849.5 (May 2015), pp. 469-483. ISSN: 18749399. DOI: 10 . $1016 / j$.bbagrm.2014.06.002. URL: http: / / linkinghub . elsevier.com/retrieve/pii/s1874939914001540

[135] Vladimir N. Uversky, Christopher J. Oldfield, and A. Keith Dunker. "Intrinsically Disordered Proteins in Human Diseases: Introducing the D2 Concept". In: Annual Review of Biophysics 37.1 (May 2008), pp. 215-246. ISSN: 1936-122X. DOI: $10.1146 /$ annurev . biophys . 37.032807.125924. URL: https://www.annualreviews.org/ doi/10.1146/annurev.biophys.37.032807.125924

[136] Vladimir Vacic, Christopher J. Oldfield, Amrita Mohan, Predrag Radivojac, Marc S. Cortese, Vladimir N. Uversky, and A. Keith Dunker. "Characterization of Molecular Recognition Features, MoRFs, and Their Binding Partners". In: Journal of Proteome Research 6.6 (June 
2007), pp. 2351-2366. ISSN: 1535-3893. DOI: 10 . 1021/pr0701411. URL: https://doi.org/10.1021/pr0701411

[137] Björn Wallner and Arne Elofsson. "Pcons5: combining consensus, structural evaluation and fold recognition scores". en. In: Bioinformatics 21.23 (Dec. 2005), pp. 4248-4254. ISSN: 1367-4803. DOI: $10.1093 /$ bioinformatics/bti702. URL:https://academic.oup.com/ bioinformatics/article/21/23/4248/195222

[138] Minglei Wang and Gustavo Caetano-Anollés. "The Evolutionary Mechanics of Domain Organization in Proteomes and the Rise of Modularity in the Protein World". English. In: Structure 17.1 (Jan. 2009), pp. 66-78. ISSN: 0969-2126. DOI: $10.1016 / j$.str.2008.11.008. URL: http: / / wWw. cell.com/structure/abstract/S09692126(08)00456-5

[139] Martin Weigt, Robert a White, Hendrik Szurmant, James a Hoch, and Terence Hwa. "Identification of direct residue contacts in proteinprotein interaction by message passing." In: Proceedings of the National Academy of Sciences of the United States of America 106.1 (Jan. 2009), pp. 67-72. DOI: $10.1073 /$ pnas . 0805923106 . URL: http: // www . pubmedcentral . nih.gov/articlerender. fcgi? artid=2629192\&tool=pmcentrez\&rendertype=abstract

[140] Travis J Wheeler, Jody Clements, and Robert D Finn. "Skylign: a tool for creating informative, interactive logos representing sequence alignments and profile hidden Markov models." In: BMC bioinformatics 15.1 (Jan. 2014), pp. 7-7. DOI: 10 .1186/1471-2105-15-7. URL: http: / / www . pubmedcentral.nih.gov/articlerender. fcgi ? artid $=3893531 \&$ tool $=$ pmcentrez \& rendertype $=$ abstract.

[141] James C. Whisstock and Arthur M. Lesk. "Prediction of protein function from protein sequence and structure". en. In: Quarterly Reviews of Biophysics 36.3 (Aug. 2003), pp. 307-340. ISSN: 1469-8994, 00335835. DOI: $10.1017 /$ S0033583503003901, URL: https: / / wWw. cambridge . org / core / journals / quarterly-reviews of - biophysics/article/prediction- of - proteinfunction - from - protein - sequence - and - structure / 1327F2FD00C0CF05497AC2575AB8D2F1.

[142] S.P. Wilkinson and A. Grove. "Ligand-responsive transcriptional regulation by members of the MarR family of winged helix proteins". In: Current Issues in Molecular Biology 8.1 (2006), pp. 51-62.

[143] N. R. Voss, M. Gerstein, T. A. Steitz, and P. B. Moore. "The Geometry of the Ribosomal Polypeptide Exit Tunnel". In: Journal of Molecular Biology 360.4 (July 2006), pp. 893-906. ISSN: 0022-2836. DOI: $10.1016 /$ 
j.jmb.2006.05.023, URL: http://www.sciencedirect.com/ science/article/pii/s002228360600605X.

[144] Marat M. Yusupov, Gulnara Zh Yusupova, Albion Baucom, Kate Lieberman, Thomas N. Earnest, J. H. D. Cate, and Harry F. Noller. "Crystal Structure of the Ribosome at $5.5 \AA$ Resolution". en. In: Science 292.5518 (May 2001), pp. 883-896. ISSN: 0036-8075, 1095-9203. DOI: 10 . 1126 / science . 1060089 , URL: http : / / science. sciencemag.org/content/292/5518/883.

[145] Adam Zemla. "LGA: a method for finding 3D similarities in protein structures". In: Nucleic Acids Research 31.13 (July 2003), pp. 3370-3374. ISSN: 0305-1048. URL: http: / / www . ncbi.nlm.nih.gov/pmc/ articles/PMC168977/

[146] Yang Zhang and Jeffrey Skolnick. "Scoring function for automated assessment of protein structure template quality". en. In: Proteins: Structure, Function, and Bioinformatics 57.4 (Dec. 2004), pp. 702-710. ISSN: 1097-0134. DOI: 10 . 1002 / prot . 20264. URL: http : / / onlinelibrary.wiley.com/doi/10.1002/prot. 20264/ abstract.

[147] O. Östling, K. J. Johanson, E. Blomquist, and E. Hagelqvist. "Dna Damage in Clinical Radiation Therapy Studied by Microelectrophoresis in Single Tumour Cells a Preliminary Report". In: Acta Oncologica 26.1 (Jan. 1987), pp. 45-48. ISSN: 0284-186X. DOI: 10 . 3109/ 02841868709092977, URL: https : / / doi . org / 10.3109/ 02841868709092977 . 



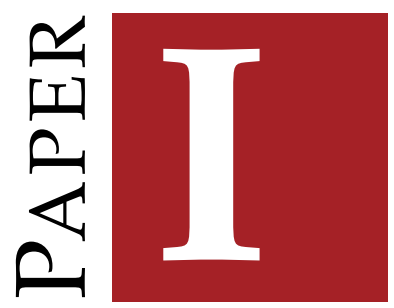

Sara Helander, Meri Montecchio, Robert Pilstål, Yulong Su, Jacob Kuruvilla, Malin Elvén, Javed M.E. Ziauddin, Madhanagopal Anandapadamanaban, Susana Cristobal,

Patrik Lundström, Rosalie C. Sears, Björn Wallner, and Maria Sunnerhagen. "Pre-Anchoring of Pin1 to Unphosphorylated c-Myc in a Fuzzy Complex Regulates c-Myc Activity". en. In: Structure 23.12 (Dec. 2015), pp. 2267-2279. ISSN: 09692126. DOI: $10.1016 / j . s t r .2015 .10 .010$ URL:

http://linkinghub.elsevier.com/retrieve/pii/S0969212615004499 (visited on $02 / 01 / 2016)$ 



\section{Structure}

\section{Pre-Anchoring of Pin1 to Unphosphorylated c-Myc in a Fuzzy Complex Regulates c-Myc Activity}

Graphical Abstract

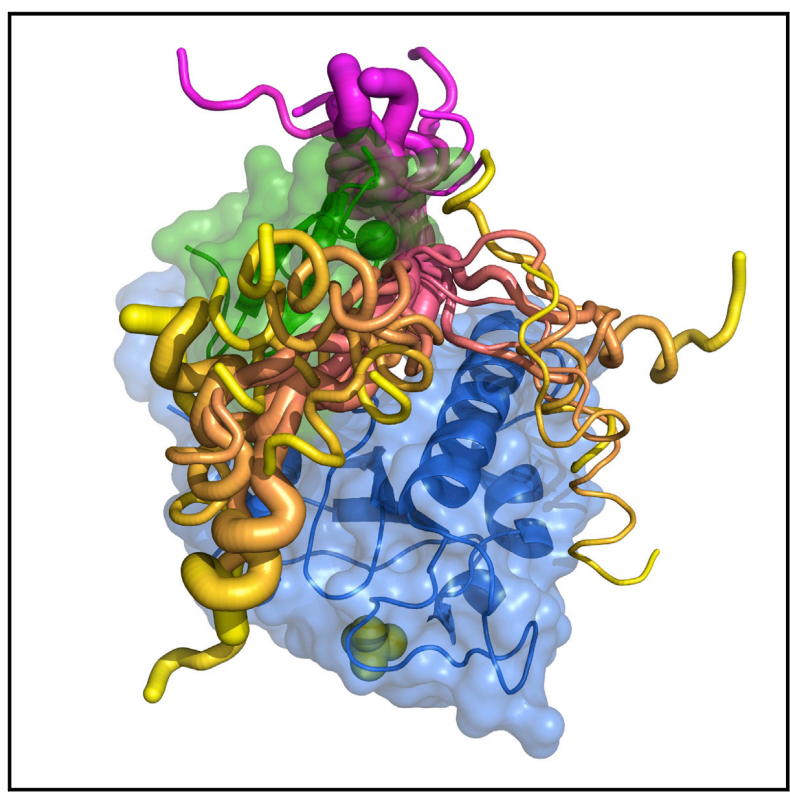

Highlights

- Pin1 binds unphosphorylated Myc distant from the isomerization target site

- Binding site mutations trap Myc in a stable, transcriptionally less active state

- A Myc-Pin1 "fuzzy complex" is described by NMR and molecular simulations

- The first structure of Pin1 in complex with a long, disordered target peptide

\section{Authors}

Sara Helander, Meri Montecchio, Robert Pilstål, ..., Rosalie C. Sears,

Björn Wallner, Maria Sunnerhagen

\section{Correspondence}

maria.sunnerhagen@liu.se

\section{In Brief}

Using an integrated structural biology approach, Helander et al. provide insights into how the structural ensemble of a disordered Myc region specifically binds to the regulatory protein Pin1 to govern cell growth and differentiation. 


\section{Pre-Anchoring of Pin1 to Unphosphorylated c-Myc in a Fuzzy Complex Regulates c-Myc Activity}

Sara Helander, ${ }^{1,5,7}$ Meri Montecchio, ${ }^{1,5}$ Robert Pilstål, ${ }^{2,5}$ Yulong Su, ${ }^{3,5}$ Jacob Kuruvilla, ${ }^{4}$ Malin Elvén, ${ }^{1,6}$ Javed M.E. Ziauddin, ${ }^{1}$ Madhanagopal Anandapadamanaban, ${ }^{1}$ Susana Cristobal, ${ }^{4}$ Patrik Lundström, ${ }^{1}$ Rosalie C. Sears, ${ }^{3}$ Björn Wallner, ${ }^{2}$ and Maria Sunnerhagen ${ }^{1, *}$

${ }^{1}$ Division of Chemistry, Department of Physics, Chemistry and Biology, Linköping University, 58183 Linköping, Sweden 2Division of Bioinformatics, Department of Physics, Chemistry and Biology, Linköping University, 58183 Linköping, Sweden ${ }^{3}$ Department of Molecular and Medical Genetics, Oregon Health and Science University, Portland, OR 92739, USA

${ }^{4}$ Department of Clinical and Experimental Medicine, Cell Biology, Faculty of Health Science Linköping University, 58183 Linköping, Sweden ${ }^{5} \mathrm{Co}$-first author

6Present address: Department of Clinical Sciences, Dermatology and Venereology, Lund University, 22184 Lund, Sweden

7Present address: Division of Drug Research, Department of Medical and Health Sciences, Linköping University, SE-581 85 Linköping Sweden

${ }^{*}$ Correspondence: maria.sunnerhagen@liu.se

http://dx.doi.org/10.1016/j.str.2015.10.010

\section{SUMMARY}

Hierarchic phosphorylation and concomitant Pin1mediated proline isomerization of the oncoprotein c-Myc controls its cellular stability and activity. However, the molecular basis for Pin1 recognition and catalysis of c-Myc and other multisite, disordered substrates in cell regulation and disease is unclear. By nuclear magnetic resonance, surface plasmon resonance, and molecular modeling, we show that Pin1 subdomains jointly pre-anchor unphosphorylated c-Myc ${ }_{1-88}$ in the Pin1 interdomain cleft in a disordered, or "fuzzy", complex at the herein named Myc Box 0 (MBO) conserved region N-terminal to the highly conserved Myc Box I (MBI). Ser62 phosphorylation in $\mathrm{MBI}$ intensifies previously transient $\mathrm{MBI}$ Pin1 interactions in $\mathrm{C}-\mathrm{Myc}_{1-88}$ binding, and increasingly engages Pin $1_{\text {PPlase }}$ and its catalytic region with maintained $\mathrm{MBO}$ interactions. In cellular assays, MB0 mutated c-Myc shows decreased Pin1 interaction, increased protein half-life, but lowered rates of Myc-driven transcription and cell proliferation. We propose that dynamic Pin1 recognition of MB0 contributes to the regulation of c-Myc activity in cells.

\section{INTRODUCTION}

Among the most fundamental processes in cell biology is the regulation of the cell cycle and, thereby, cell growth. A key player in this process is the peptidyl-prolyl isomerase Pin1 that has been shown to function as a molecular timer by acting as a switch of various cell-signaling processes (Liou et al., 2011; Lu et al., 2007). One of the targets of Pin1 is the proto-oncogenic transcription factor c-Myc, which is a universal regulator of cell growth, apoptosis, and proliferation in both normal and tumor cells (Lin et al., 2012; Meyer and Penn, 2008; Nie et al., 2012).
While the pro-proliferative properties of c-Myc are activated by phosphorylation of Ser62, its cellular stability and degradation are controlled by subsequent phosphorylation at Thr58, both sites being located in the conserved Myc Box I (MBI) region of its $\mathrm{N}$-terminal transcriptional activation domain (TAD) (Hann, 2006). The time window of phosphorylation and dephosphorylation at these two sites is affected by Pin1-mediated cis-trans isomerization of the Ser62-Pro motif, thereby regulating specific interactions of c-Myc with modifying kinases, predominantly acting on trans substrates (Brown et al., 1999; Lu et al., 2002a) and phosphatases, which control the pathway of C-Myc activation and ubiquitin-mediated degradation (Figure 1A) (Farrell and Sears, 2014; Sears, 2004), and which have been shown to act on cis substrates (Werner-Allen et al., 2011). Pin1 also facilitates the dynamic binding of c-Myc to target gene promoters, enhancing association with transcriptional co-activators and transcriptional activation of target genes, thus potentiating c-Myc's oncogenic activity (Farrell et al., 2013). The importance of regulatory coupling c-Myc activation with its subsequent degradation in maintaining normal cell growth is evidenced by cancer-associated mutations at or near Thr58 and Ser62 that result in maintaining c-Myc in its activated, Ser62-phosphorylated state (Bahram et al., 2000; Wang et al., 2011), and many regulators of c-Myc that bind to this region are themselves either oncogenes or tumor suppressors (Tu et al., 2015). Although Pin1 promotes c-Myc degradation in normal cells, this activity is uncoupled in cancer cells where both proteins have oncogenic activities and are overexpressed, and Pin1 functions only as a c-Myc co-activator (Farrell et al., 2013). Potential uncoupling mechanisms involve reduced Axin1 function, which scaffolds c-Myc degradation (Arnold et al., 2009; Zhang et al., 2012b) and Pin1's downregulation of Fbw7, an E3 ligase controlling degradation of a number of oncoproteins including c-Myc (Min et al., 2012).

Pin1 comprises two independently folded subdomains: Pin1 ww (residues 6-39) and Pin1 pplase (residues 50-163), connected by a flexible linker (Bayer et al., 2003; Jacobs et al., 2003; Ranganathan et al., 1997). The catalytic proline isomerization activity toward pSer/pThr-Pro motifs entirely resides in 
A

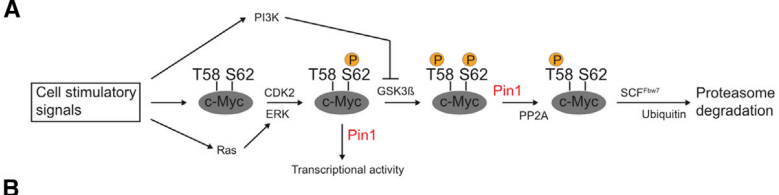

B

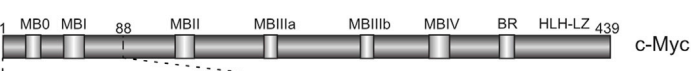

C
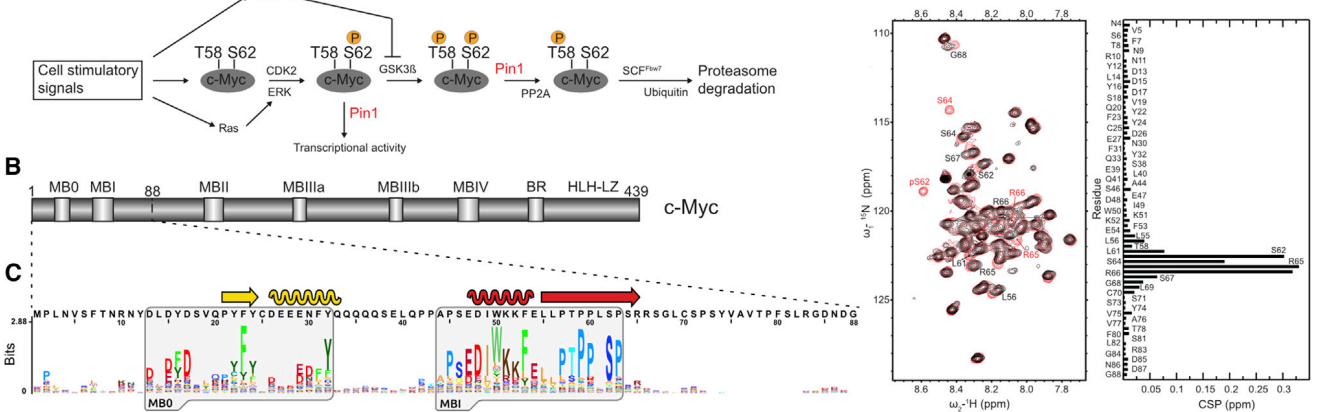

Figure 1. Phosphorylation and Conserved Patterns in c-Myc

(A) A schematic of the biological context of Myc and Pin1 interactions as described in the Introduction. Myc phosphorylations at T58 and S62 annotated with encircled $P$.

(B) Sequence outline, indicating the here-proposed MBO (c-Myc $\left.{ }_{15-33}\right)$ together with previously identified Myc boxes (MBI, MBII, MBIIla, MBIIlb, and MBIV), the basic region (BR), and the helix-loop-helix leucine zipper (HLH-LZ).

(C) A sequence logo representing c-Myc residues 1-74 based on a Hidden Markov Model of Myc and generated as described in Experimental Procedures. High stacks of few, dominant amino acid letters signify conservation; $\mathrm{MBO}$ and $\mathrm{MBl}$ are highlighted. Graphical representations (curl and arrow) respectively indicate the stacks of few, dominant amino acid letters signify conservation; $\mathrm{MBO}$ and $\mathrm{MBl}$ are highlight
location of previously identified transient secondary structure (Andresen et al., 2012).

location of previously identified transient secondary structure (Andresen et al., 2012).
(D) Overlaid HSQC NMR spectra of unphosphorylated c-Myc $1-88$ (black) and pSer62-c-Myc $1-88$ (red). Peaks that display CSPs >0.05 ppm on Ser62 phosphorylation are labeled.

(E) CSPs on phosphorylation for all assigned $\mathrm{C}-\mathrm{Myc}_{1-88}$ amide resonances. See also Figure S1.

Pin1pplase (Lu et al., 1999; Ranganathan et al., 1997). The Pin1 $1_{\text {PPlase }}$ alone binds weakly to native, phosphorylated peptide targets $\left(K_{D}>390 \mu \mathrm{M}\right)($ De et al., 2012; Verdecia et al., 2000), with significant affinities $\left(K_{d}\right.$ range $\left.0.5-90 \mu \mathrm{M}\right)$ observed only for peptides selected from library screens (Duncan et al., 2011: Namanja et al., 2011; Verdecia et al., 2000) or designed inhibitors (Namanja et al., 2011; Zhang et al., 2012a). Pin1ww binds pSer/ pThr-Pro-containing peptides with variable affinities $\left(K_{d}>\right.$ $7 \mu \mathrm{M}$ ) (Lu et al., 1999; Verdecia et al., 2000), preferably in the extended, trans conformation (De et al., 2012; Namanja et al., 2011; Verdecia et al., 2000; Wintjens et al., 2001). In intact Pin1, the two domains create an interdomain cleft, distant from the Pin $1_{\text {PPlase }}$ active site, where phosphorylated target peptides $\left(K_{d} 5-80 \mu \mathrm{M}\right)$ (Verdecia et al., 2000) as well as buffer components such as polyethylene glycol (PEG) bind in well-defined conformations (reviewed in Matena et al., 2013). Pin1 ww binding at phosphorylated pSer/pThr-Pro sites has been proposed to increase the local effective concentration of substrate and activity on neighboring phosphorylated sites (Jacobs et al., 2003; Lu et al., 1999), to facilitate substrate transfer (De et al., 2012; Lu et al., 1999; Wintjens et al., 2001), and/or to sequester trans conformations away from the active site, thereby providing directionality to the cis-trans conversion (De et al., 2012; Namanja et al., 2011; Wintjens et al., 2001). Still, the functional reason for binding of targeted peptides to an interdomain cleft distant from the Pin1 active site remains unresolved.

Recent studies suggest that collaborative and possibly allosteric mechanisms jointly involve both Pin1 domains in target binding (reviewed in Peng, 2015). Binding of shorter peptide substrates and small molecules to Pin1 has been shown to affect interdomain mobility and linker dynamics (Jacobs et al., 2003), and increased affinity and isomerization of phosphorylated peptides binding to Pin1 1 pplase has been shown in the presence of PEG-induced transient domain interactions (Matena et al., 2013). Interactions between the two domains have been shown to allosterically affect the isomerization activity by an internal dynamic circuit through the Pin $1_{\text {Pplase }}$ interior (Namanja et al., 2011), as well as through residues in the domain interface (Wilson et al., 2013), both recently supported by molecular simulations (Guo et al., 2015). However, to understand how the dualdomain protein Pin1 acts on its longer, multiply phosphorylated, and often intrinsically disordered substrates (Lu and Zhou, 2007), the interaction with such substrates needs to be studied in structural and dynamic detail, but as yet such studies have not been achieved.

We recently characterized the structural and dynamic properties of the most $\mathrm{N}$-terminal part of the c-Myc TAD domain (c-Myc ${ }_{1-88}$ ), by nuclear magnetic resonance (NMR) and surface plasmon resonance (SPR), detailing also its dynamic and multivalent interactions with the tumor suppressor Bin1 (Andresen et al., 2012). We found that c-Myc $\mathrm{C}_{1-88}$ contains two transiently ordered regions: the well-characterized $\mathrm{MBI}$ region and a less studied, more $\mathrm{N}$-terminal region conserved in $\mathrm{C}-, \mathrm{N}-$, and L-Myc (Cowling and Cole, 2006; Legouy et al., 1987) (Figures $1 \mathrm{~B}$ and $1 \mathrm{C})$. While $\mathrm{MBI}$ is a well-known c-Myc interaction site, and comprises the phosphorylation sites directing c-Myc stability (Meyer and Penn, 2008), so far no clear functional role has been attributed to the transiently ordered region $\mathrm{N}$-terminal to $\mathrm{MBI}$.

Here, we show for the first time how a longer Pin1 substrate, C-Myc ${ }_{1-88}$, interacts with both domains of Pin1, and how this interaction is affected by phosphorylation. By SPR, NMR, and

2268 Structure 23, 2267-2279, December 1, 2015 @2015 Elsevier Ltd All rights reserved 
A

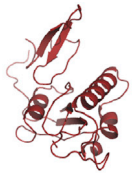

Pin1
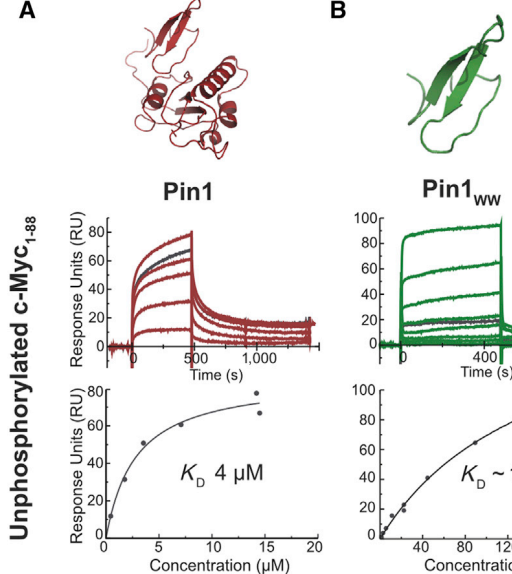

Pin1 ${ }_{w w}$

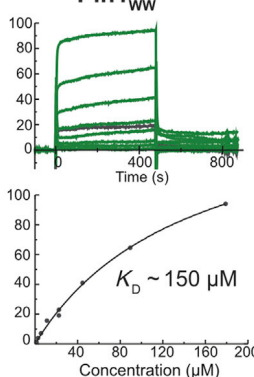
$\begin{array}{ccc}40 & 120 & 160 \\ \text { Concentration }(\mu \mathrm{M}) & 200\end{array}$

E

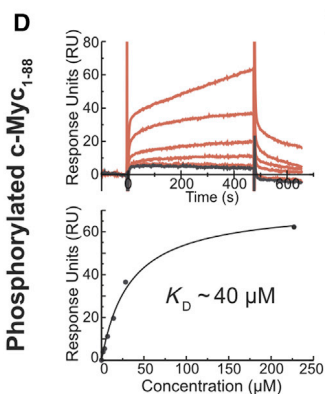

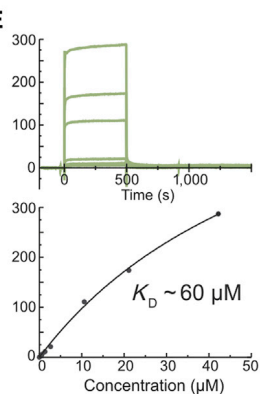

Concentration $(\mu \mathrm{M})$

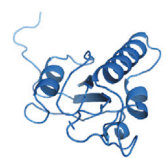

Pin1 $1_{\text {PPlase }}$

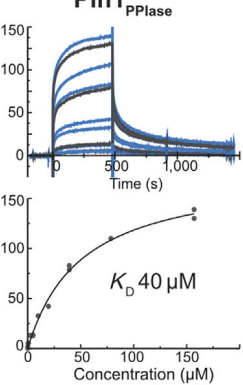

$\mathbf{F}$

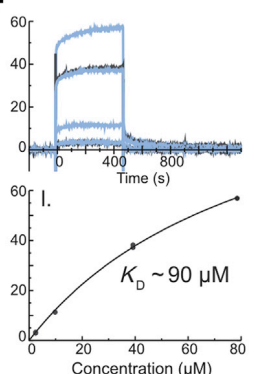

Figure 2. Evaluation of $\mathrm{c}-\mathrm{Myc}_{1-88}-\mathrm{Pin} 1 \mathrm{Af}-$ finities by Surface Plasmon Resonance Sensorgrams and 1:1 Langmuir fits from steadystate evaluation show binding of Pin1 (red), Pin1 1 wr (green), Pint ww (grion) (blue) to both unphosphoyled (A-C) and phosphorlated $\mathrm{C}-\mathrm{Myc}_{1-88}(\mathrm{D}-\mathrm{F})$. For all measurements C-Myc $\mathrm{C}_{1-88}$ was immobilized and Pin1, Pin1ww, or Pin1 PPlase were injected over the surface. SPR experiments are further detailed in Figure S2.

S1A-S1D). Chemical-shift perturbations (CSPs) were confined to the wellconserved $\mathrm{MBI}$ region (Figures $1 \mathrm{D}$ and $1 \mathrm{E})$. The transverse relaxation rates for c-Myc ${ }_{1-88}$ are concentration dependent (Figures $\mathrm{S} 1 \mathrm{H}$ and $\mathrm{S} 1 \mathrm{I}$ ), which agrees with monomer exchange with a small amount of higher molecular weight oligomer not observed by sample inspection or in gel-filtration experiments (Andresen et al., 2012). However, the overall decreased $R_{2}$ relaxation rates for pSer62-c-Myc $\mathrm{C}_{1-88}$ compared with $\mathrm{C}-\mathrm{Myc}_{1-88}$ at the same concentration suggest that the additional negative charge introduced by phosphorylation reduces internal interactions (Figure S1F). Both ${ }^{15} \mathrm{~N}-\mathrm{R}_{1}$ and $\left\{{ }^{1} \mathrm{H}\right\}-{ }^{15} \mathrm{~N}-$ nuclear Overhauser effect (NOE) relaxation of pSer62-c- $\mathrm{Myc}_{1-88}$ correspond well to results obtained for $\mathrm{C}-\mathrm{Myc}_{1-88}$ at similar concentrations (Figures S1E-S1G), suggesting that the intrinsic disorder with transiently structured regions identified

cellular assays, we show that the transiently ordered, unphosphorylated c-Myc region comprising c-Myc residues 13-32, which we henceforth refer to as "Myc Box 0" (MB0; Figures 1B and $1 \mathrm{C}$ ), serves as a dynamic anchoring site for Pin1 on $\mathrm{C}-\mathrm{Myc}_{1-88}$, both in the absence and presence of Ser62 phosphorylation. Molecular simulations, restricted by experimental data, show how Pin1 WW and PPlase domains jointly bind the $\mathrm{MBO}$ region in a dynamic complex, thus facilitating MBI interaction with the Pin1 active site. Our results suggest that Pin 1 binding to MBO affects the cellular activity window of the c-Myc oncoprotein.

\section{RESULTS}

Intrinsic Disorder Is Retained in pSer62-c-Myc ${ }_{1-88}$ To study the Ser62 phosphorylated state of C-Myc, we performed phosphorylation of $\mathrm{C}^{-\mathrm{Myc}_{1-88}}$ in vitro with active CDK2 kinase in complex with CyclinA2, which is known to phosphorylate Ser62-Pro63 in vivo (Hydbring et al., 2010) and in trans (Brown et al., 1999). Specific and near-complete pSer62-c-Myc ${ }_{1-88}$ phosphorylation was confirmed by mass spectrometry and NMR, in agreement with the extended properties of the MBI region (Andresen et al., 2012) (Figures

et al. 2012) is maintained upon Ser62

for C-Myc ${ }_{1-88}$ (Andre

Intact Pin1 Binds Unphosphorylated c-Myc $\mathbf{1}_{1-88}$

We used SPR to investigate the binding of Pin1 and its subdomains to $\mathrm{C}-\mathrm{Myc}_{1-88}$ and pSer62-c-Myc $\mathrm{M}_{1-88}$ (Figures 2, S2A, and S2B). Since Pin1 is believed to primarily bind at phosphorylated protein sites (Liou et al., 2011; Lu and Zhou, 2007), we were surprised to find that intact Pin1 binds $\mathrm{C}-\mathrm{Myc}_{1-88}$ with an apparent $K_{\mathrm{D}}$ of $4 \mu \mathrm{M}$ (Figures 2A and S2B). This Pin1 binding affinity is of similar or higher affinity as previously observed Pin1 binding to phosphorylated peptides, but several orders of magnitude higher than for unphosphorylated Ser/Thr-Pro motifs (Verdecia et al., 2000). Lack of significant Pin1 binding to C-Myc $46-69$ (Figure S2A) indicated that the primary binding site for Pin1 to c-Myc $1-88$ is located distant from the Ser62-Pro motif. Both Pin $1_{\mathrm{ww}}$ and Pin $1_{\text {pplase }}$ subdomains were consistently found to bind $\mathrm{c}-\mathrm{Myc}_{1-88}$ with more than 10-fold weaker affinities compared with intact Pin1 (Figures 2B, 2C, and S2B), suggesting both Pin1 subdomains jointly contribute to the higher c-Myc ${ }_{1-88}$ affinity of the intact protein. SPR measurements of Pin1 affinities to pSer62-c-Myc ${ }_{1-88}$ showed difficulties in reaching saturation and anomalous binding effects at higher concentrations, which 
A

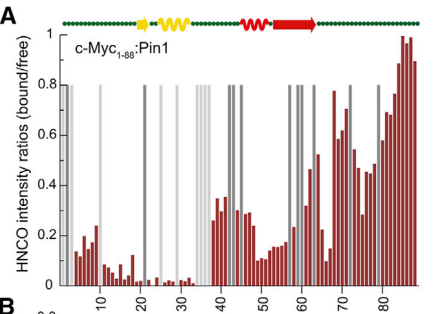

B
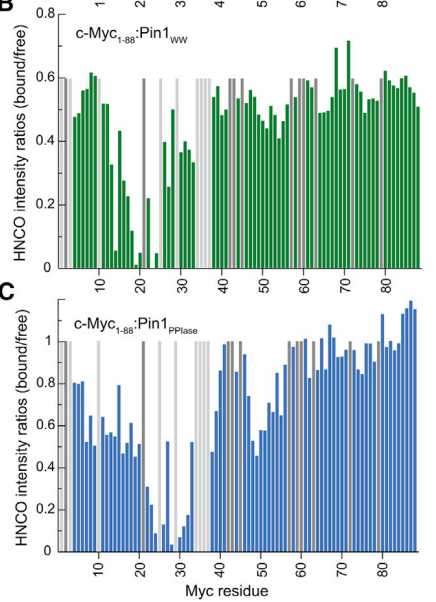

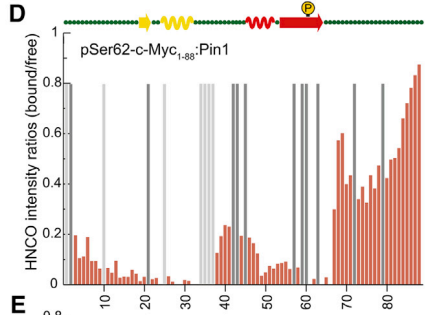

E ${ }_{0.8}$

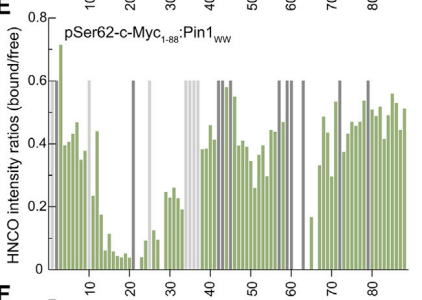

$\mathbf{F}$

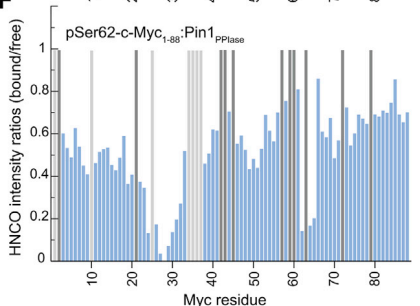

Figure 3. NMR Analysis of $\mathrm{C}-\mathrm{Myc}_{1-88}$ PerResidue Interactions with Pin1 and Its Subdomains

HNCO peak intensity ratios between Pin1-bound and free states were derived for $\mathrm{C}-\mathrm{Myc}_{1-88}$ when and free states we denived for $\mathrm{CMyC}_{1-88}$ when

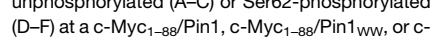
(D-F) at a c-Myc ${ }_{1-88} / \mathrm{Pin}_{1}, \mathrm{C}-\mathrm{Myc}_{1-88} / \mathrm{Pin} 1_{\mathrm{ww}}$, or C-
$\mathrm{Myc}_{1-88} / \mathrm{Pin}_{\text {Pplase }}$ ratio of $1: 2$, with estimated $\mathrm{Myc}_{1-88} / \mathrm{Pin} 1_{\mathrm{PPlase}}$ ratio of $1: 2$, with estimated
saturation levels ranging from $58 \%$ to $96 \%$ (see Experimental Procedures). Prolines (dark gray; give no signal in $\mathrm{HNCO}$ ) and residues lacking data due to missing assignment or overlap (light gray) are represented by solid histogram bars. Binding to and $F$ ) is color- Prod as in Figut and $F$ ) is color-coded as in Figure 2. Gain/loss of interactions in the bound state leads to decreased/ increased peak intensity ratios, respectively. Graphical representations (curl and arrow, respectively) indicate the location of previously identified transient secondary structure (Andresen et al., 2012). The phosphorylation site at $\mathbf{S} 62$ is indicated with encircled P. See also Figure S3.

(Figure 3), CSPs were very small or nonexistent (Figure S3). While peak intensities in general are lowered upon interaction due to slower molecular tumbling in the complex state, further line broadening leading to reduced intensities can occur as a result of chemical exchange between bound and free states, and/or due to chemical exchange between multiply bound states (Bozoky et al., 2013a, 2013b; Lukhele et al., 2013; Mittag et al.,

limited the accuracy (Figures 2D-2F). Notably, however, the phosphorylated substrate may bind the active Pin1 enzyme in several modes with different affinities, due to cis-trans isomerization of pSer62-Pro (De et al., 2012; Jacobs et al., 2003; Namanja et al., 2011). In agreement with this, isothermal titration calorimetry measurements indicate multiple binding events for intact Pin1 binding to pSer62-cMyc ${ }_{1-88}$, with $K_{d} s$ ranging from 10 to $100 \mu \mathrm{M}$ (Figures S2C-S2E), i.e. in the same range as measured for other phosphorylated peptides (Verdecia et al., 2000). The higher pSer62-c-Myc ${ }_{1-88}$ apparent affinities for intact Pin1 compared with its subdomains alone indicate joint subdomain binding contributions also for phosphorylated c-Myc $\mathrm{C}_{1-88}$. Finally, while full kinetic analysis was not feasible due to the rapid on- and off-rates in binding, we consistently observed elevated off-rates by visual inspection for Pin1 1 PPlase binding to pSer62$\mathrm{C}-\mathrm{Myc}_{1-88}$ compared with unphosphorylated c-Myc $\mathrm{C}_{1-88}$ (Figures $2 \mathrm{C}$ and $2 \mathrm{~F}$ ), in agreement with increased isomerization turnover of phosphorylated substrates.

Pin1 Binds c-Myc ${ }_{1-88}$ in a Dynamic Complex Anchored at the MBO Region

By NMR, we further investigated c-Myc-Pin1 interactions on a per-residue level by titrating ${ }^{15} \mathrm{~N}$-labeled $\mathrm{c}-\mathrm{Myc}_{1-88}$ and pSer62-c- Myc $_{1-88}$ with unlabeled Pin1. We found that while for specific c-Myc residues resonance intensities were significantly reduced and not recovered on saturating the binding equilibrium
2008). In the latter case, the signal is not recovered in the bound state due to continued chemical exchange (Bozoky et al., 2013b), which is in agreement with our observations for Pin1/ $\mathrm{C}^{-} \mathrm{Myc}_{1-88}$ complexes. Furthermore, we detected no ${ }^{15} \mathrm{~N}$ CarrPurcell-Meiboom-Gill dispersions, showing that line broadening due to chemical exchange occurs on a faster timescale than can be probed by these experiments. These observations are in agreement with the formation of dynamically disordered (Forman-Kay and Mittag, 2013), or "fuzzy" (Fuxreiter and Tompa, 2012), complexes.

Since c-Myc ${ }_{1-88}$ exhibits low chemical-shift dispersion (Andresen et al., 2012) and very minor CSPs in the bound state (Figure S3), HNCO intensity ratios (Mittag et al., 2008) were primarily used to characterize the interaction of $\mathrm{c}-\mathrm{Myc}_{1-88}$ to intact Pin1 and its subdomains (Figure 3). Binding of intact Pin1 to non-phosphorylated $\mathrm{c}-\mathrm{Myc}_{1-88}$ results in major loss of signal intensity primarily in the conserved, transiently ordered $\mathrm{MB} 0$ region, but also affects the $\mathrm{MBI}$ region (Figures $3 A$ and $3 D$ ). Intact Pin1 binding to pSer62-c-Myc ${ }_{1-88}$ more clearly affects the phosphorylation site and flanking residues, and perturbations extend into the transiently helical and conserved $\mathrm{MBI}$ region around $\mathrm{C}-\mathrm{Myc}_{1-88}-\mathrm{Trp50}$, while the C-terminal part remains comparatively unperturbed (Figure 3D). Binding of either Pin1 $1_{w w}$ or Pin1 1 pplase resulted in drastically lowered $\mathrm{HNCO}$ intensity ratios in the MBO region of $\mathrm{c}-\mathrm{Myc}_{1-88}$, suggesting that both Pin1 subdomains target this region

2270 Structure 23, 2267-2279, December 1, 2015 @2015 Elsevier Ltd All rights reserved 
A
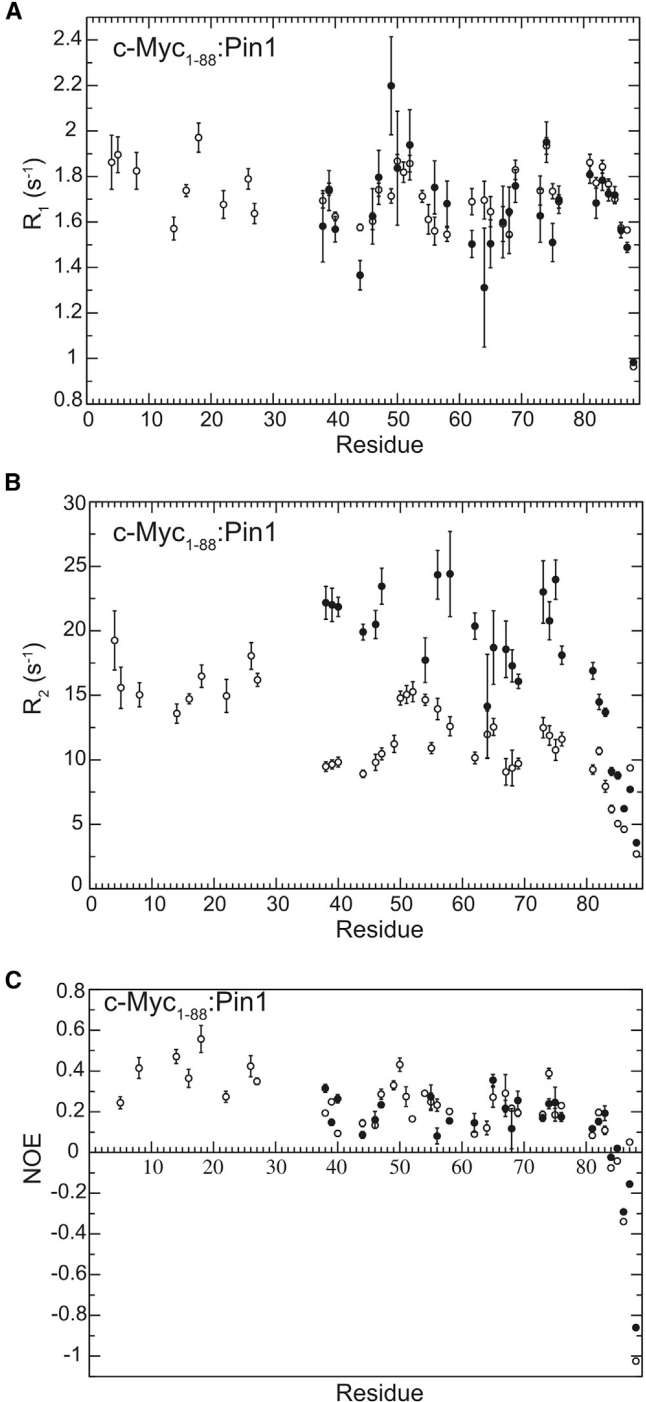

Figure 4. NMR Relaxation Analysis of $\mathrm{c}-\mathrm{Myc}_{1-88}$ Binding to Pin1 Relaxation parameters of apo $\mathrm{c}-\mathrm{Myc}_{1-88}$ (open circles) and c-Myc $\mathrm{M}_{1-88}$ (filled circles) in the presence of $2 \mathrm{~mol}$ equivalents of Pin1 ( $98 \%$ saturation). Uncertainties in R1 and R2 were estimated using the jackknife method, and in the heteronuclear NOE as the standard deviation of all permutations of peak ratios in duplicate experiments (Ahlner et al., 2013).

(A) $R_{1}$ relaxation rates

(B) $\mathrm{R}_{2}$ relaxation rates.

(C) $\left\{{ }^{2} \mathrm{H}\right\}-{ }^{15} \mathrm{~N}-\mathrm{NOE}$.
(Figures 3B and 3C). Pin1ww primarily affects c-Myc residues 16-YDSVQPYFY-23 (Figure 3B), while Pin1 pplase affects the aromatic residues in the 20-PYFY-23 motif as well as conserved residues 29-ENFY-32 slightly C-terminal to the Pin1 ww binding motif (Figure $3 \mathrm{C}$ ). The proline-aromatic 20PYFY-23 pattern affected by both Pin1 subdomains is also found in Pin1 binding peptides selected from library screens, both in forward (CTGIPWLYC; Duncan et al., 2011) and reverse sequence orientation (Pintide: WFYpSPFLE; Lu et al., 1999; Verdecia et al., 2000).

On phosphorylation, the binding pattern of Pin1 subdomains to pSer62-c-Myc ${ }_{1-88}$ alters significantly (Figures $3 \mathrm{E}$ and $3 \mathrm{~F}$ ). While interactions to MB0 are maintained, both Pin ${ }_{\text {ww }}$ and Pin1 1 PPlase binding now also leads to severely reduced HNCO peak intensity ratios at c-Myc $\mathrm{M}_{1-88}-$ pSer62 and adjacent residues (Figures $3 \mathrm{E}$ and $3 \mathrm{~F}$ ). Furthermore, binding of both C-Myc ${ }_{1-88}$ and pSer62-c-Myc ${ }_{1-88}$ to Pin1 results in more extensive effects than would be predicted from Pin1 subdomain binding alone (Figures $3 \mathrm{~A}$ and $3 \mathrm{D}$ ). This suggests that both Pin1 domains jointly bind C-Myc, which agrees with the increased affinity for intact Pin1 compared with its subdomains as observed by SPR (Figure 2). Furthermore, the NMR results suggest that the conserved, transiently structured $\mathrm{MB0}$ region in $\mathrm{C}-\mathrm{Myc}_{1-88}$ is central for Pin1 interactions with $\mathrm{c}-\mathrm{Myc}_{1-88}$ irrespective of the phosphorylation state of Ser62.

To further investigate $\mathrm{c}-\mathrm{Myc}_{1-88}$ dynamics upon Pin1 binding, we evaluated ${ }^{15} \mathrm{~N}-\mathrm{R}_{1},{ }^{15} \mathrm{~N}-\mathrm{R}_{1}$, and $\left\{{ }^{1} \mathrm{H}\right\}-{ }^{15} \mathrm{~N}-\mathrm{NOE}$ relaxation experiments for the saturable unphosphorylated $\mathrm{c}-\mathrm{Myc}_{1-88}-\mathrm{Pin} 1$ complex. Although excessive line broadening in the MB0 region limited the evaluation, the residues that showed sufficient signal intensity for analysis had ${ }^{15} \mathrm{~N}-\mathrm{R}_{1}$ relaxation rates similar to those of free $\mathrm{C}-\mathrm{Myc}_{1-88}$, indicating retained mobility of flanking regions

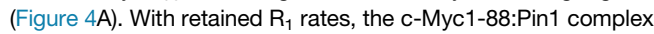
displays higher ${ }^{15} \mathrm{~N}-\mathrm{R}_{2}$ relaxation rates compared with free

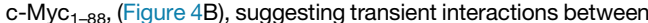
Pin1 and regions flanking the $\mathrm{MBO}$ anchor site in agreement with decreased $\mathrm{HNCO}$ intensity ratios in the same regions (Figure 3). NOE relaxation rates in the $\mathrm{c}-\mathrm{Myc}_{1-88}-\mathrm{Pin} 1 \mathrm{complex}$ are similar to those in free $\mathrm{c}-\mathrm{Myc}_{1-88}$ and correspond well to earlier characterized transient secondary elements (Andresen et al., 2012), suggesting that $\mathrm{C}-\mathrm{Myc}_{1-88}$ transient structure is retained upon Pin1 binding (Figure 4C). Taken together, NMR relaxation measurements show retained intrinsic disorder in $\mathrm{C}-\mathrm{Myc}_{1-88}$ when binding Pin1, signifying a fuzzy complex (Fuxreiter and Tompa, 2012).

Pin1 Recognition Pattern Is Altered by $\mathbf{M y c}_{1-88}$ Phosphorylation

To obtain more detailed molecular insight on the extent of Pin1 that interacts with c-Myc, we mapped spectral changes in ${ }^{15} \mathrm{~N}$, ${ }^{13} \mathrm{C}$-labeled Pin1 on addition of unlabeled $\mathrm{C}-\mathrm{Myc}_{1-88}$ and pSer62-c-Myc ${ }_{1-88}$ by NMR. For both unphosphorylated and phosphorylated C-Myc, small but significant CSPs were observed (Figures 5, S4A, and S4B). While CSPs only suggest that the chemical environment around the concerned backbone $\mathrm{NH}$ groups has changed, the most likely reasons are a direct binding with an interaction partner or an indirect conformational change due to this interaction. 
B
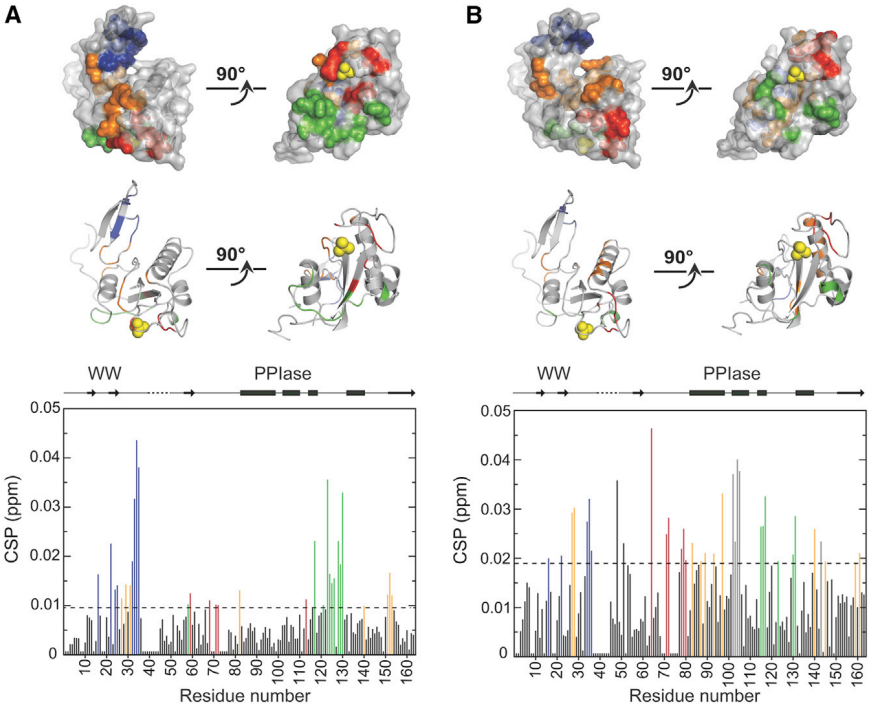

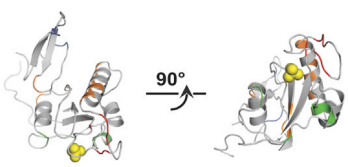

ww

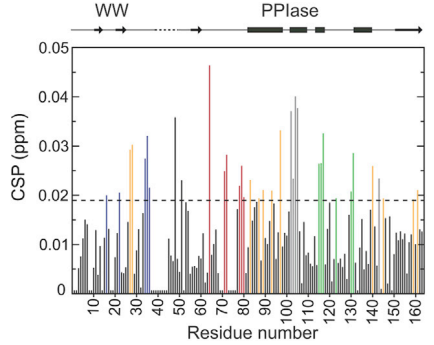

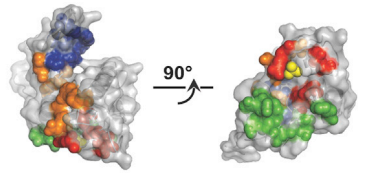

Figure 5. NMR Mapping of Pin1 Interactions with c-Myc ${ }_{1-88}$ and pSer62-c-Myc ${ }_{1-88}$

Surface, secondary structure representations, and charts of the CSPs of Pin1, interacting with c-Myc $\quad$ (A) and PSer62-c-Myc (B), respecC-Myci-88 (A) and pSero2-C-Myc $1-88$ (B), respectively. color coded through (A) and (B) as follows: residues in Pin1ww (blue), subdomain interface (orange), Pin1 $1_{\text {pPlase }}$ catalytic loop and active site (red), Pin1 $1_{\text {PPlase }}$ substrate recognition and binding (green). The sulfate group from the crystal structure $1 \mathrm{PIN}$, represented by yellow spheres, indicates the proposed Pin1 active site. See also Figure S4.

the active-site loop (Figure 5, red) via highly affected Pin1-His64 (Figure 5), which was previously found to be highly perturbed in the binding of both cis- and trans-restricted inhibitors (Namanja et al., 2011). Notably, Pin1 helix1, the interdomain interface, and the WW pocket are also fundamental parts of the second allosteric pathway ("Path 2") identified by Guo et al. (2015). Pin1 residues proposed to be involved in substrate recog-

In the unphosphorylated $\mathrm{c}-\mathrm{Myc}_{1-88}$ :Pin1 interaction, a nearcontinuous surface of CSP-displaying residues is formed by Pin1ww residues centered around Trp34 (Figure 5A, blue), and, extending toward the Pin $1_{\mathrm{PPlase}}$ active site, by residues facing the cleft between the two domains (Figure 5A, orange). This suggests a Myc binding surface involving the interdomain cleft, which agrees with Pin1ww and Pin1 $1_{\text {PPlase }}$ jointly binding the C-Myc MBO region (Figure 3). In the WW domain, affected residues correspond well to those observed in crystal structures to be involved in PEG and phosphopeptide binding (Matena et al., 2013; Namanja et al., 2011; Ranganathan et al., 1997; Verdecia et al., 2000). In addition, a clear pattern of CSPs is observed for Pin $1_{\text {Pplase }}$ residues involved in substrate recognition and proline ring placement (Figure 5A; green) while weak CSPs are observed for residues in the active-site loop (Figure 5A; red). Interestingly, the Pin1 interdomain interface and PPlase core region affected by $\mathrm{C}-\mathrm{Myc}_{1-88}$ includes many residues in the "Path 1" proposed to mediate allosteric interdomain regulation (Guo et al., 2015).

The pSer62-c-Myc ${ }_{1-88}:$ Pin1 interaction results in a more extensive CSP pattern, which increasingly affects Pin 1 residues involved in domain interactions and catalysis (Figure 5B). The effects of Pin1 interaction with pSer62-c-Myc ${ }_{1-88}$ now extends from the Trp34 region to involve WW residues His27 and lle28 lining the interdomain cleft, as well as Ala140 facing these residues from the PPlase domain. Notably, Pin1-lle28 was recently shown to be a key residue in regulating substrate binding affinity and isomerase activity by means of its interdomain contact position (Wilson et al., 2013). Furthermore, facing the Pin $1_{\text {ww }}$ domain, the Pin1 $1_{\text {pplase }}$ helix 1 (residues 82-98), which was not affected by unphosphorylated $\mathrm{C}-\mathrm{Myc}_{1-88}$, now shows significant CSPs (Figure 5, orange). Helix1 connects to nition and proline ring placement (Namanja et al., 2011) are differently affected: residues $115-117$ and $130-131$ at the edge of the Pin $1_{\text {pPlase }}$ phosphate binding groove are involved in recognition of phosphorylated c-Myc while unphosphorylated c-Myc affects the surface-exposed $\beta$ strand and loop structure including residues 120-130 (Figure 5; green). Specifically, Pin1 residues 178 and A116, which show enhanced flexibility on ligand binding in previous studies as a possible reflection of substrate recognition (Namanja et al., 2011), are both highly affected by pSer62-c-Myc ${ }_{1-88}$ binding but not by binding to unphosphorylated $\mathrm{c}-\mathrm{Myc}_{1-88}$. Finally, residues $102-105$, close to the hydrophobic patch where a second PEG molecule was observed in the Pin1 crystal structure deposited as 1PIN (Ranganathan et al., 1997), now show CSPs, suggesting altered interactions at this site (Figure 5, gray). The interaction of Pin1 with c-Myc could be further modulated by phosphorylation, since two of three important phosphorylation sites in Pin1 (Ser16 and Ser71; Hariharan and Sussman, 2014; Lee et al., 2011; Lu et al., 2002b) are involved in c-Myc interactions with both phosphorylated and unphosphorylated states (Figure 5).

A comparison between HNCO peak intensity ratios for Pin1, in the presence of either $\mathrm{c}-\mathrm{Myc}_{1-88}$ or pSer62-c-Myc ${ }_{1-88}$, reveals a general and uniform reduction of peak intensity ratios for both folded domains of Pin1, in contrast to the residue-specific effects observed for C-Myc ${ }_{1-88}$ (Figures S4C and S4D). This suggests that the two Pin1 subdomains, which in the free state move independently of each other (Bayer et al., 2003; Jacobs et al., 2003), experience an increased $\tau_{c}$ as an effect of joint tumbling of the two domains on binding either phosphorylated or unphosphorylated $\mathrm{c}-\mathrm{Myc}_{1-88}$, which again supports joint c-Myc binding by Pin $1 \mathrm{ww}$ and Pin $1_{\text {PPlase }}$ subdomains.

2272 Structure 23, 2267-2279, December 1, 2015 @2015 Elsevier Ltd All rights reserved 
Ensemble Models Describe the Fuzzy Myc-Pin1 Complex

To outline the possibilities for deriving a structural model fo the c-Myc-Pin1 interaction, we jointly assessed all our experimental data (Figures 2, 3, 4, and 5). SPR measurements showed significant binding of Pin1 to unphosphorylated CMyc outside of the Pin1-targeted MBI (Figures 2, S2A, and S2B), and, in agreement NMR mapping of HNCO intensity ratios, suggests that Pin1 primarily binds to the $\mathrm{MBO}$ region (c-Myc ${ }_{10-35}$ ) (Figure 3). We focused on investigating how MBO may interact with Pin1, and how this might affect binding of the unphosphorylated MBI region, since binding to pSer62-cMyc $_{1-88}$ seems structurally less resolvable due to the presence of multiple interconverting bound states (Figures 2 and S2C-S2E).

To visualize the structural space accessible to a c-Myc $\mathrm{C}_{1-88^{-}}$ Pin1 dynamic complex that satisfies our experimental data, we first modeled $\mathrm{c}-\mathrm{Myc}_{10-35}$ in complex with Pin1 and then extended this ensemble to include the entire $\mathrm{c}-\mathrm{Myc}_{10-70}$ region, using Rosetta docking and loop modeling protocols (see Experimental Procedures). The docking was guided by experimental constraints derived from HNCO intensity data from $\mathrm{C}-\mathrm{Myc}_{1-88}$ binding to Pin1, Pin1 ${ }_{\mathrm{ww}}$ and Pin $1_{\text {pplase, as }}$ well as from Pin1 CSPs on $\mathrm{C}-\mathrm{Myc}_{1-88}$ binding (see Experimental Procedures). The resulting conformations were clustered using a $3-\AA$ r root-mean-square deviation radius cutoff, and the largest clusters, representing a majority of the docked conformations, were sorted based on combined physical and constraint energies (Figures S6). From each of these clusters, the lowest-energy representative that best satisfied the experimental constraints was included in the ensemble describing the bound complexes (Figure 6 and Movie S1).

The resulting models show a wide ensemble of c-Myc $\mathrm{C}_{10-35}$ conformations favorably interacting with both Pin $1_{w w}$ and Pin $1_{\text {PPlase }}$ by means of the MB0 region (Figures $6 \mathrm{C}, 6 \mathrm{D}, 6 \mathrm{G}$ and $6 \mathrm{H}$; Figure $\mathrm{S} 5 \mathrm{~A}$; Movie $\mathrm{S1}$ ). A preferred direction of binding of MBO in the interdomain cleft is evident and is introduced by the experimental constraints, since without that contribution to the energy function the bias almost disappears (Figure S5B). In the full $\mathrm{c}-\mathrm{Myc}_{10-70}$ model, the extent of the linker between MBO and MBI generously allows for unphosphorylated $\mathrm{MBI}$ to interact in a multitude of ways with the Pin1 1 pplase active-site region (Figures $6 \mathrm{E}-6 \mathrm{G}, 6 \mathrm{I}$, and $\mathrm{S6}$ ), in full agreement with experimental data (Figures 3, 4, and 5) Notably, our NMR relaxation data for unphosphorylated $\mathrm{C}-\mathrm{Myc}_{1-88}$ show that interactions between Pin1 and regions flanking the MBO anchor site are dynamically transient, in contrast to the multistate, fuzzy Pin1 binding of MBO (Figures 3 and 4). Thus, the entire ensemble of Pin1-bound $\mathrm{c}-\mathrm{Myc}_{1-70}$ will also include Myc conformations that are anchored only at $\mathrm{MBO}$, leaving the $\mathrm{MBI}$ region disordered and accessible to phosphatases and kinases.

\section{Mutations in MBO Affect Critical Myc Regulatory} Functions

The biophysical and computational data suggest that the conserved $\mathrm{MBO}$ region forms a primary interaction site for Pin1 to unphosphorylated $\mathrm{c}-\mathrm{Myc}_{1-88}$. To evaluate the importance of this interaction in cells and for c-Myc's cellular func- tions, we generated point mutations in the MB0 coding region, targeting C-Myc-20-PYFY-23 in human corresponding to cMyc-20-PYFI-23 in murine, and created murine alanine c-Myc mutants 20-PAAA, 20-PAAI, and 20-AAAI. Consistent with the hypothesis that these residues are important for Pin1 to anchor on c-Myc (Figure 3), our co-immunoprecipitation (colP) experiments reproducibly showed more than $50 \%$ reduction in Pin1's ability to bind to MB0 mutated c-Myc compared with wild-type (WT), with AAAl the lowest affinity observed (Figure 7A). The cellular data agree well with SPR experiments performed with three corresponding human $\mathrm{C}-\mathrm{Myc}_{1-88}$ mutants, c-Myc-20PYFY/AAAA-23, -PYFY/PAAY, and -PYFY/AAFY. Compared with WT c-Myc ${ }_{1-88}$, the c-Myc-20-AAAA-23 mutant displays a lower affinity toward human Pin1, $15 \mu \mathrm{M}$, compared with $4 \mu \mathrm{M}$ for the WT construct (Figures S7A and S7D). Measurements were also performed with the other two mutants cMyc-20-PYFY/PAAY and c-Myc-20-PYFY/AAFY, which were also able to bind Pin1, but showed reduced affinity (Figures S7B-S7D). In addition, the mutants are phosphorylated at S62 with similar or higher levels compared with WT c-Myc, suggesting that the reduced affinity is not caused by lack of c-Myc S62 phosphorylation, and consistent with a role for Pin1 in facilitating PP2A-mediated S62 dephosphorylation (Yeh et al., 2004) (Figure 7B). We also observed an increase in protein stability in the MBO mutants reflected by a prolonged protein half-life following inhibition of protein synthesis by cycloheximide treatment (Figure 7C) and by a higher steadystate level when transfecting equal amounts of plasmids to the WT (Figure 7D, western blot). The increased stability of the MB0 mutants is likely due to downregulation of Pin1's facilitating C-Myc degradation caused by reduced interaction (Yeh et al., 2004).

Since in addition to facilitating c-Myc Ser62 dephosphorylation and degradation, Pin1 initially stimulates c-Myc DNA binding and target gene activation (Farrell et al., 2013), we tested the effects of the c-Myc-20-PYFI mutations on cMyc transcriptional activity. We measured the luciferase signal driven by the canonical c-Myc binding sequence, 4xE-Box-luc, following expression of c-Myc WT or MBO mutants. Mutants with disruption of c-Myc-20-PYFI consistently showed decreased ability to drive luciferase signal compared with WT c-Myc (Figure 7D). The c-Myc-20-PYFI mutants also have significantly decreased interaction with endogenous targets genes, E2F2 and Nucleolin, measured by quantitative ChIP (Figure 7E). Interestingly, the degree to which the mutants display impaired activities coordinates well with their abilities to interact with Pin1 (Figure 7A), suggesting that Pin1 is the main regulator of the c-Myc-20-PYFI domain and its effects on c-Myc activity. To test the role of c-Myc20-PYFI in cell proliferation, we conducted colony formation assays in REF52 cells. As shown in Figure 7F, the ability of cells to form colonies with the AAAl mutant and Ras was dramatically decreased compared with cells with WT Myc and Ras, highlighting the importance of the c-Myc-20-PYFI domain for c-Myc's pro-proliferative function. We find that several pro-proliferative transcription factors that are Pin1 targets (Liou et al., 2011) contain a PYFY-type motif N-terminal to their pSer-Pro sites, suggesting a more general role of this motif in Pin1-regulated pro-proliferative targets (Figure S7E). 

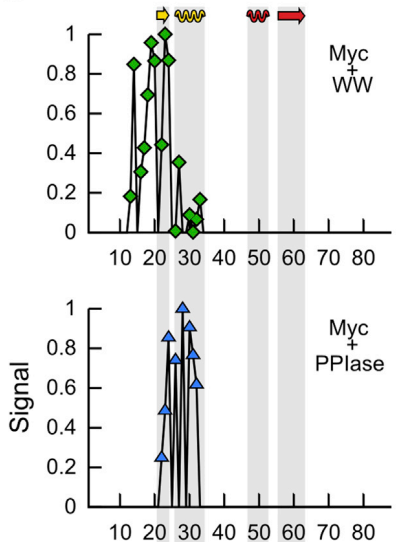

1020304050607080

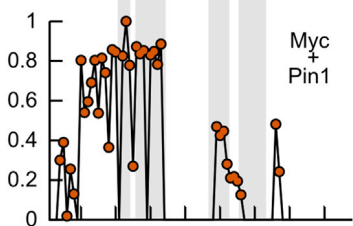

1020304050607080 Residue

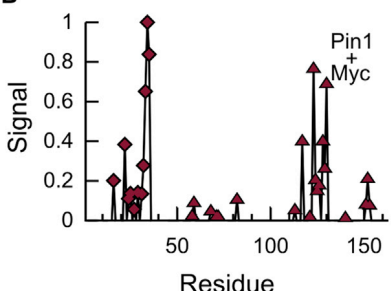

C
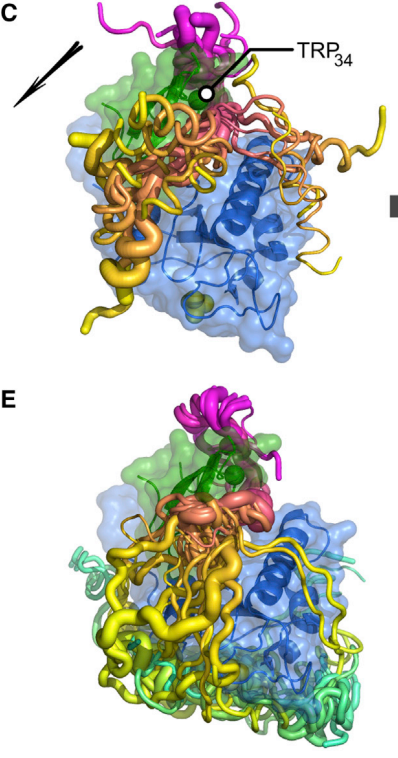

D

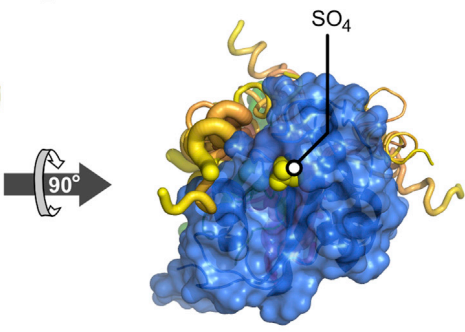

F

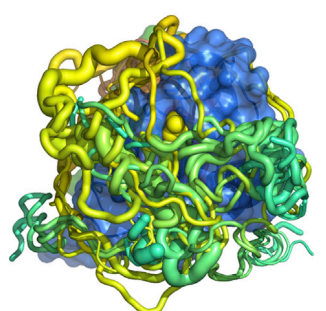

G

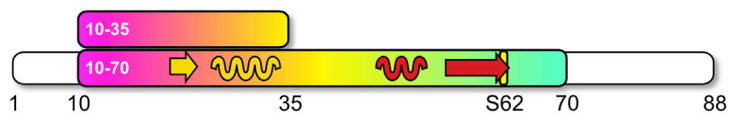

H

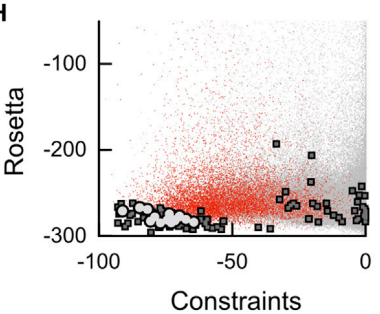

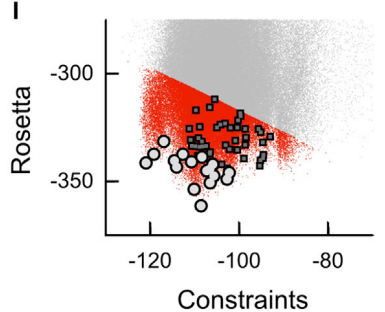

Figure 6. Ensemble Model of the Interaction Between c-Myc and Pin1

(A) Filtered and rescaled signals used to guide the Rosetta Monte Carlo simulation from HNCO ratios for c-Myc ${ }_{1-88}$ versus Pin1 ww $_{\text {(top), }}$ C-Myc 1-88 versus $_{1}$ Pin1 $1_{\text {pPlase }}$ (middle), and c-Myc $1-88$ versus full Pin1 (bottom).

(B) Plot of rescaled signals from Pin1 versus C-Myc ${ }_{1-88} \Delta$ CSPs.

(C) A structural representation of the MB0-anchored Myc-Pin1 fuzzy complex, as presented by the ensemble of lowest-energy c-Myc ${ }_{10-35}$-Pin1 complex conformations from the 15 top-scoring clusters, visualized as ribbon representations of the c-Myc $\mathrm{C}_{10-35}$ backbone superimposed on PDB:1PIN. The cross-section area of the ribbon is scaled to the relative size of the cluster. The black arrow indicates the $\mathrm{N}$ - to C-terminal c-Myc ${ }_{10-35}$ binding direction as defined in Figure S5B. (D) Bottom view of the complex ensemble with $\mathrm{SO}_{4}$ in the superimposed $1 \mathrm{PIN}$ structure bound to the active site, represented by yellow spheres.

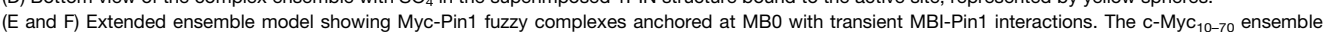
comprises the 17 top-scoring clusters, visualized in the same manner as the c-Myc $\mathrm{C}_{10-35}$ model.

(G) Relative position of the modeled Myc fragments on C-Myc $1-88$ with the same coloring gradient of C-Myc ${ }_{10-35}$ and C-Myc $10-70$ as in (C) to (F); transient secondary structure elements and the Ser62 phosphorylation site are indicated.

(H) The experimental constraint score versus the Rosetta standard score is plotted as dots for all c-Myc $\mathrm{C}_{10-35}$-Pin1 models; red dot if the model belongs to a cluster and gray otherwise. The lowest-energy representative is shown for each cluster irrespective of size (black squares), with the 15 highest-scoring clusters highlighted (gray circle).

(I) Scatterplot of $\mathrm{C}-\mathrm{Myc}_{10-70}$ clusters and -Pin1 models; same visualization as in $(\mathrm{H})$ but with the 17 highest-scoring clusters highlighted.

See also Figure S5.

2274 Structure 23, 2267-2279, December 1, 2015 @2015 Elsevier Ltd All rights reserved 
A
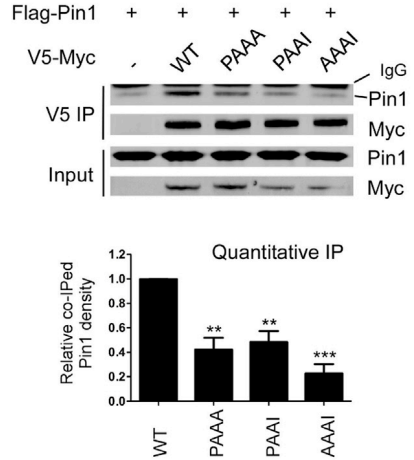

B

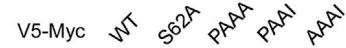
pS62 Myc 내 Total Myc $=-\mathrm{m}$

C $\begin{array}{lllll}\mathrm{CHX}(\mathrm{hr}) & 0 & 0.5 & 1 & 2\end{array}$ V5-Myc b-Actin

WT $t(1 / 2)=33.4 \pm 3.2 \mathrm{~min}$ V5-Myc b-Actin

AAAl $t(1 / 2)=49.8 \pm 4.4 \mathrm{~min}$

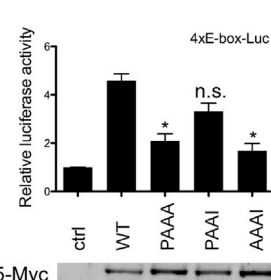

E

V5-Myc qChIP

E2F2

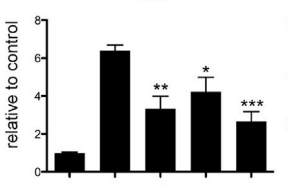

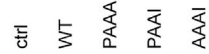

Nucleolin

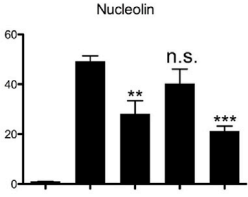

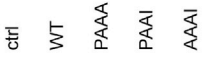

Figure 7. Mutations in MBO Decrease Pin1 Affinity and Regulate c-Myc activity

Reduced binding affinity with Pin1, less transcriptional activity, and reduced pro-proliferation ability is observed for C-Myc-20-PYFI mutants. (A) CofP of with indicated plasmids using anti-V5 antibody for immunoprecipitation and anti-Flag antibody for detection of Co-immunoprecipitated Pin 1 (bottom band). Co-immunoprecipitated Pin1 band densities were quantified after normalization to input, immunoprecipitated c-Myc level, and controls. (B) Western analysis of lysates from HEK293 expressing indical pressing indicated plasmids, V5-inmunoprecipitated C-Myc were used for S62 phosphorylation analysis, S62A serves as negative control.

(C) Western analysis of lysates from HEK293 transfected with equal amounts of V5-Myc plasmids (top: WT; bottom: AAAI mutant). Four independent experiments were used to quantify halflife.

(D) $4 \times \mathrm{xE}$-box driven luciferase signal detected from lysates of HEK lysates of divera lucico-rase signal dected $\checkmark 5$ plasmids or empty vector as control. Luciferase signals were adjusted based on expression levels shown in the immunoblots below.

(E) DNA binding affinity for WT and mutant V5-Myc shown by quantitative ChIP at indicated promoters. ChIP levels were adjusted by respective inputs and protein levels. Lysates of HEK203s transfected empty vector were used as control for transfected empty

(F) Colony formation assays were performed in
(REFalizan. (F) Colony formation assays were performed in
REF52 cells co-transfected with H-Ras and either WT or mutant c-Myc as indicated for up to 3 weeks.

Statistical significance relative to WT was calculated using a two-tailed t test: ${ }^{*} p<0.05 ;{ }^{* *} p<0.01$ ${ }_{* * *}<<0.001, n . s$, not significant. All quantifications $p<0.001$, n.s., not signifcant. All quantifications were based

See also Figure $\mathrm{S} 7$.

\section{DISCUSSION}

The Pin1 enzyme is well known for its capability of cis-trans isomerization of phosphorylated (p)Ser/(p)Thr-Pro target sequences, and specifically acts on intrinsically disordered substrates in cell regulation to direct their regulatory activities (Liou et al., 2011; Lu et al., 2007). However, limited light has been shed on how Pin1 interacts with longer substrates, since the present molecular knowledge predominantly has been gained from Pin1 studies of interactions with small molecules or peptides. The present work significantly advances our understanding of Pin1-substrate interactions by showing, using a wide spectrum of biophysical and cellular methods, that Pin1 recognition of its well-known target c-Myc involves pre-anchoring to an unphosphorylated conserved motif distal from the phosphorylated target site for Pin1-mediated proline isomerization, which is biologically critical and structurally significant. Specifically, we find that Pin1 binds unphosphorylated c-Myc in a Pin1 interdomain-anchored dynamic, or fuzzy, complex at a well-conserved region here designated MBO (Figure 1), and we show that such anchoring is structurally compatible with concomitant transient binding to the Pin1 1 PPlase active site region of the $\mathrm{MBI}$ (p)Ser/(p)Thr-Pro Pin1 target site(s) for cis/trans isomerization (Figure 6), in full agreement with our NMR data (Figures 3 , 4, and 5). Importantly, disturbing Pin1 binding to its unphosphorylated MBO anchor site results in impaired c-Myc transcriptional activity and reduced c-Myc function in cellular proliferation assays.

A compelling advantage of Pin1 pre-anchoring to unphosphorylated, distal site(s) would be to increase the local concentration of substrate presented to the active site and thereby further increase the catalytic efficiency of Pin1. Our study substantiates this, since we in our NMR experiments directly observe a significant recognition of the unphosphorylated $\mathrm{MB}$ region by intact Pin1 on MBO binding (Figure 3), indicating that this recognition is facilitated by an increase in local concentration due to Pin1-MB0 pre-anchoring. When $\mathrm{c}-\mathrm{Myc}_{1-88}$ is phosphorylated at Ser62, we find that Pin1 distinctly recognizes the phosphorylation site in $\mathrm{MBI}$ but still affects the $\mathrm{N}$-terminal, non-phosphorylated MBO (Figure 3), suggesting a maintained 
role of this interaction in the phosphorylated state. Furthermore, the mere localization of MBO binding to the interdomain cleft (Figures 5 and 6) may also mediate increased catalytic activity. Recent work has shown enhanced interdomain interactions in Pin 1 by small-molecule binding at the Pin1 subdomain interface (Matena et al., 2013), and critical interdomain contact residues such as lle28 have been shown to affect Pin1 catalytic activity and substrate binding affinity (Wilson et al., 2013). Notably, however, simultaneous tight binding of designed bivalent Pin1 binders to both WW and PPlase creates inhibition of Pin1 activity (Daum et al., 2007), suggesting that intrinsic dynamics in binding both Pin1 sites is required for native substrate activity.

Our results show that mutations in the MBO Pin1-anchoring region dramatically decrease Ras-dependent transformation and simultaneously show prolonged c-Myc half-life. At first glance this may appear confusing, since several stabilizing c-Myc mutations have been shown to increase cell proliferation (Chang et al. 2000; Salghetti et al., 1999). However, our results are compatible with recent data demonstrating that Pin1 isomerization at pSer62-Pro63 in c-Myc promotes c-Myc DNA binding and target gene activation (Figure 1; Farell et al., 2013) even though it can also facilitate $\mathrm{S62}$-dephosphorylation by protein phosphatase 2A and increase c-Myc turnover (Figure 1; Yeh et al., 2004; Arnold and Sears, 2006). Thus, a Myc mutant with deficient Pin1 interaction would indeed be more stable and Ser62 phosphorylated, but would not promote transcriptional and/or oncogenic activity. Since our biophysical results indicate that Pin1 binding to the MB0 site N-terminal to the c-Myc pSer62-Pro motif serves to allosterically activate Pin1 and increase the frequency of encounters between the $\mathrm{MBI}$ target sequence and the PPlase active site, it is indeed a tenable hypothesis that with disturbed MB0 pre-recognition, both the Pin1 isomerase activity and the affinity between Myc and Pin1 will be too low to be biologically efficient. This would result in a higher prevalence of Myc isomers that are less active in transcriptional activity in the Myc-accessible cellular activity window, with concomitant lowering of cell growth, as shown in our MB0 mutants.

We suggest that the biological requirement for both Pin1 subdomains to reach full Pin1 functionality is based on the biophysical requirements for joint subdomain interaction to longer substrates, which is necessary to allosterically elevate enzymatic activity and specificity to the levels required for efficient biological regulation. Recent studies show that interdomain dynamics is affected by binding of small molecules or substrate peptides/analogs to the active site and/or to the interdomain region (Bayer et al., 2003; Jacobs et al., 2003; Matena et al., 2013 Namanja et al., 2007; Wilson et al., 2013). Our data support and extend these findings by showing how Pin1 integrates recognition to both these sites in binding to a longer substrate. Importantly, we show that not only key residues, such as Pin1 ${ }_{\text {ww-lle28 }}$ and its counterpart Ala140 in Pin1 $1_{\text {PPlase }}$, but a wide range of interdomain residues in both Pin1 subdomains are differentially affected with respect to the phosphorylation state of the binding peptide (Figure 5). A recent study performed by molecular dynamics simulations proposes that the two Pin 1 domains are allosterically regulated through two pathways: first quiescent state called Path 1, and a second state (Path 2) which is sequentially activated upon substrate binding to the WW pocket (Guo et al., 2015). Interestingly, in our experi- mental work with a long substrate, we find that the CSPs in Pin1 created by the binding with $\mathrm{c}-\mathrm{Myc}_{1-88}$ resembles the Path 1 pattern, while with $\mathrm{pS62}-\mathrm{CMyc}_{1-88}$ a pattern comparable with Path 2 emerges (Figure 5). Notably, while the WW pocket is involved in binding both phosphorylated and unphosphorylated c-Myc ${ }_{1-88}$, it is only in the presence of pS62-c-Myc ${ }_{1-88}$ that the second pathway becomes active. Therefore, we propose that the presence of the phosphate in the active site represents the real trigger factor for the activation of Path 2, given joint binding of the substrate protein to the WW pocket. Thus, the allosteric communication between the two Pin1 domains is not a oneway signal initiated by Pin $1_{\text {ww }}$ binding, but it is a multistep process whereby Path 1 is activated by substrate binding to the WW domain and Path 2 by the phosphate binding the PPlase active site. Taken together, a comparison between results relating to Pin1 allostery in our experimental data and in the molecular dynamics simulations (Guo et al., 2015) reveals interesting similarities, but also different implications concerning the activation mechanism of allosteric pathways.

The highly dynamic association between non-phosphorylated c-Myc ${ }_{1-88}$ and Pin1 shows the characteristics of a fuzzy complex (Fuxreiter and Tompa, 2012): alternate conformations are allowed in the bound state, and large parts of the bound $\mathrm{c}-\mathrm{Myc}_{1-88}$ peptide have dynamic properties closely resembling the unbound state. The unphosphorylated Myc-Pin1 complex must therefore be considered as an ensemble of conformations where, although one orientation of c-Myc in the interdomain cleft is prevalent, a wide variety of chain-wrapping modes around Pin1 are allowed in the bound ensemble (Figures 6 and S5). Redistribution among multiple states within the bound ensemble could well occur on Myc phosphorylation, which could also increase the propensities for MBI binding to the Pin1 interdomain cleft, as suggested by Pin1 ${ }_{\text {ww }}$ binding to both MB0 and MBI upon Ser62 phosphorylation (Figure $3 \mathrm{E}$ ) as well as by previous crystal structures showing phosphorylated short peptides or peptide analogs bound in the interdomain cleft (Matena et al., 2013; Verdecia et al., 2000). The juggling of substrates on and off the active site, as well as the enzymatic efficiency (De et al., 2012), may thus be controlled by dynamically shifting the ensemble of interdomain Pin 1 bound states, as indicated by the requirement of both domains for efficient catalysis and by the multiple modes of Myc-Pin1 recognition identified here. Furthermore, the fuzzy complex with a flexible 30 -residue $(>60 \AA)$ linker between the $\mathrm{Myc} \mathrm{MBO}$ and $\mathrm{MBI}$ binding sites (Figure 6), together with the dynamics of the bound complex (Figure 4), will allow for facile kinase/phosphatase access to Ser/Thr-Pro sites in MBI with maintained MB0 association of Pin1 throughout the regulatory cycle (Figure 1). Our findings, together with the possible presence of distal motifs in other Pin1-dependent pro-proliferative transcription factors (Figure S7E), suggest that dynamic pre-recognition of distal motifs by Pin 1 could play a more general role in the timing of cellular events in growth and differentiation.

\section{EXPERIMENTAL PROCEDURES}

Bioinformatics Sequence Evaluation

A Profile Hidden Markov Model logo, describing sequence conservation, was constructed for $\mathrm{c}-\mathrm{Myc}_{1-88}$ as described in Supplemental Experimental Procedures. 
Protein Expression, Purification, and Phosphorylation Human Pin1 constructs for Escherichia coli overexpression of full-length Pin (1-163), Pin1 ww (6-39), and Pin1 pplase (50-163) (Bayer et al., 2003) were kindly provided by Prof. Bayer (University of Duisburg-Essen). Pin1 and Myc proteins were prepared according to previously published protocols (Andresen et al., 2012; Bayer et al., 2003) with only minor modifications (see Supplemental Experimental Procedures). Phosphorylation of $\mathrm{c}-\mathrm{Myc}_{1-88}$ in vitro was performed using CDK2/CyclinA2 kinase (Promega) and analyzed by liquid chromatography-tandem mass spectrometry as detailed in Supplementa Experimental Procedures.

\section{NMR Spectroscopy}

All experiments were performed at $15^{\circ} \mathrm{C}$ using a 600-MHz Varian INOVA spec-

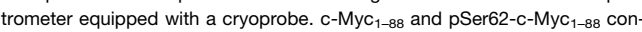
centrations were as low as permitted by the sensitivity of the experiments to limit possible effects of self-association: $80-115 \mu \mathrm{M}$ in HNCO titrations, $180 \mu \mathrm{M}$ in Pin 1 titrations, and $250 \mu \mathrm{M}$ in relaxation measurements. Samples for HNCOs and relaxation experiments of the Myc-Pin1 complex contained $1 \mathrm{~mol}$ equivalent of $\mathrm{C}-\mathrm{Myc}_{1-88}$ (unphosphorylated or phosphorylated) an 2 mol equivalents of Pin1, Pin1 ${ }_{w w}$, or Pin1pplase. Details concerning recorde spectra, data processing, and relaxation analysis are described in the Supplespectra, data processing, and relaxation analysis are described in the Supple(c) 20602 (C-Myct Myc $\left.{ }_{1-88}\right)$ and BMRB: 26663 (pSer62-c-Myc $1-88$ ).

Surface Plasmon Resonance Experiments

SPR measurements were performed at $25^{\circ} \mathrm{C}$ using a Biacore 3000 instrument. The sensorgrams were all adequately fitted by a 1:1 Langmuir model, which was thus employed to derive apparent $K$ s for interactions of intact Pin1 and or C-Myc ${ }_{1-88}$ mutants. Details on measurement conditions and sensorgram evaluations are given in Supplemental Experimental Procedures and Figure S2.

Structural Modeling Guided by Experimental Constraints As detailed in Supplemental Experimental Procedures, Rosetta FlexPepDock (Raveh et al., 2011) was used to dock c-Myc ${ }_{10-35}$ and c-Myc $\mathrm{M}_{40 / 45-70}$ fragments to Pin 1 in a Monte Carlo approach. First, $\mathrm{C}-\mathrm{Myc}_{10-35}$ was modeled using constraints from HNCO experiments involving either of the separate Pin 1 ww and Pin1 1 domains. The ensemble model was then extended Pin $1 \mathrm{ww}$ and Pin 1 pPlase domains. The ensemble model was then extended
to include MBI by first modeling c-Myc $\mathrm{My}_{40-70}$ and $\mathrm{c}-\mathrm{Myc}_{45-70}$ separately using the same procedure, but with constraints from HNCO experiments involving both Pin1 domains. By joining these docked ensembles with those of c-Myc ${ }_{10-35}$ by loop modeling (Wang et al., 2007; Qian et al., 2007) and subsequent refinement, a joint $\mathrm{C}-\mathrm{Myc}_{10-70} \mathrm{MB} 0+\mathrm{MBI}$ ensemble was obtained. The docking was guided by experimental constraints derived from significant signals in CSP and HNCO experiments. The threshold signicant signas in cSP and 作 intact Pin 1 binding were set at below 0.4 and 0.2 , respectively, to qualitatively reflect the observed binding pattern. Above (Pin1 $\triangle \mathrm{CSP}$ ) and below (c-Myc1-88 HNCOs) the thresholds, each set of signals was linearly rescaled between 0.0 and 1.0

Cell Culture, Transfection, Immunoprecipitations, and Biologica Assays

Cell culture, Myc immunoprecipitation, and luciferase assays were performed as previously described (Yeh et al., 2004; Arnold et al., 2009; Farrell et al., 2013). ChIP experiments were performed based on previous studies (Farrell et al., 2013) with slight modifications. The immunoprecipitated DNA were purified with the QIAquick PCR purification kit and used for QPCR analysis with specified primers as described previously (Farrell et al., 2013). Antibodies used for western blotting were anti-V5 mouse monoclon antibody (Invitrgen), anti-FLAG M2 mose monoclonat antibody (Sigma), (ab78318). For colony formation assays, expression vectors containing $\mathrm{c}$-Myc (WT or mutant) and $\mathrm{H}$-ras ${ }^{\mathrm{G} 12 \mathrm{~V}}$ were used for transfections in REF52 cells. $72 \mathrm{hr}$ after transfection, cells were replated at low density. Following replating, cells were maintained in DMEM containing $4 \%$ fetal bovine serum, and the colonies were visualized by staining with crystal violet blue 2 weeks ater.

Detailed protocols are provided in Supplemental Experimental Procedures.

\section{SUPPLEMENTAL INFORMATION}

Supplemental Information includes Supplemental Experimental Procedures, seven figures, and one movie and can be found with this article online at http://dx.doi.org/10.1016/j.str.2015.10.010.

\section{AUTHOR CONTRIBUTIONS}

Conceptualization: M.S., R.S., and S.H. Methodology: M.A., P.L., R.P., M.S., R.S., Y.S., and B.W. Investigation: S.H., M.M., R.P., Y.S., J.M., M.E., J.K., and M.S. Writing-original draft: M.S., S.H., M.M., R.P., R.S., Y.S., and B.W. Reviewing and editing: M.S., M.A., M.M., R.S., R.P., and B.W. Supervision: M.S., M.A. S.C. P. L R.S and B.W. Project administration: M.S. Co-first authors equally Contibuted MyC NMR and SPR (S.H.), Pint titration calorimetry (M.M.), modeling (R.P.), and cell biology (Y.S.).

\section{ACKNOWLEDGMENTS}

We acknowledge Drs. Cecilia Andresen, Veronika Csizmok, Julie Forman-Kay, and Linda Penn for critical discussion, Dr. Peter Bayer for sharing human Pin1, Pin1 1 , and Pin1 expression constructs, Marie Roth for protein producor access to NMR Whe Knut and Alice Wallenberg Foundation for equipment grants to LiU and SNC. Project funding is acknowledged from the Swedish Cancer Foundation (M.S.), the Swedish Child Cancer Foundation (M.S.), the Carl Trygger foundation (M.S.), the LiU Cancer Research Network (M.S.), the Swedish Research Council (S.C., P.L., M.S., B.W.), and the NCI R01s CA129040 and CA100855 (R.S.).

Received: April 22, 2015

Revised: September 30, 2015

Accepted: October 1, 2015

Published: November 19, 2015

\section{REFERENCES}

Ahlner, A., Carlsson, M., Jonsson, B.-H., and Lundström, P. (2013). PINT: a software for integration of peak volumes and extraction of relaxation rates. J. Biomol. NMR 56, 191-202.

Andresen, C., Helander, S., Lemak, A., Farès, C., Csizmok, V., Carlsson, J., Penn, L.Z., Forman-Kay, J.D., Arrowsmith, C.H., Lundström, P., and Sunnerhagen, M. (2012). Transient structure and dynamics in the disordered c-Myc transactivation domain affect Bin1 binding. Nucleic Acids Res. 40, 6353-6366.

Arnold, H.K., and Sears, R.C. (2006). Protein phosphatase 2A regulatory subunit B56alpha associates with c-myc and negatively regulates c-myc accumulation. Mol. Cell. Biol. 26, 2832-2844.

Arnold, H.K., Zhang, X., Daniel, C.J., Tibbitts, D., Escamilla-Powers, J., Farrell, A., Tokarz, S., Morgan, C., and Sears, R.C. (2009). The Axin1 scaffold protein promotes formation of a degradation complex for c-Myc. EMBO J. 28, 500-512.

Bahram, F., von der Lehr, N., Cetinkaya, C., and Larsson, L.G. (2000). c-Myc hot spot mutations in lymphomas result in inefficient ubiquitination and decreased proteasome-mediated turnover. Blood 95, 2104-2110.

Bayer, E., Goettsch, S., Mueller, J.W., Griewel, B., Guiberman, E., Mayr, L.M., and Bayer, P. (2003). Structural analysis of the mitotic regulator $\mathrm{hPin} 1$ in solution: insights into domain architecture and substrate binding. J. Biol. Chem. 278, 26183-26193.

Bozoky, Z., Krzeminski, M., Chong, P.A., and Forman-Kay, J.D. (2013a). Structural changes of CFTR $R$ region upon phosphorylation: a plastic (n) 4407-4416.

Bozoky, Z., Krzeminski, M., Muhandiram, R., Birtley, J.R., Al-Zahrani, A., Thomas, P.J., Frizzell, R.A., Ford, R.C., and Forman-Kay, J.D. (2013b). 
Regulatory R region of the CFTR chloride channel is a dynamic integrator of phospho-dependent intra- and intermolecular interactions. Proc. Natl. Acad. Sci. USA 110, E4427-E4436.

Brown, N.R., Noble, M.E.M., Endicott, J.A., and Johnson, L.N. (1999). The structural basis for specificity of substrate and recruitment peptides for cy clin-dependent kinases. Nat. Cell Biol. 1, 438-443.

Chang, D.W., Claassen, G.F., Hann, S.R., and Cole, M.D. (2000). The c-Myc transactivation domain is a direct modulator of apoptotic versus proliferative signals. Mol. Cell. Biol. 20, 4309-4319,

Cowling, V.H., and Cole, M.D. (2006). Mechanism of transcriptional activation by the Myc oncoproteins. Semin. Cancer Biol. 16, 242-252.

Daum, S., Lücke, C., Wildemann, D., and Schiene-Fischer, C. (2007). On the benefit of bivalency in peptide ligand/pin1 interactions. J. Mol. Biol. 374, 147-161. De, S., Greenwood, A.I., Rogals, M.J., Kovrigin, E.L., Lu, K.P., and Nicholson, L.K. (2012). Complete thermodynamic and kinetic characterization of the isomer-specific interaction between Pin1-WW domain and the amyloid precurso protein cytoplasmic tail phosphorylated at Thr668. Biochemistry 51, 85838596.

Duncan, K.E., Dempsey, B.R., Killip, L.E., Adams, J., Bailey, M.L., Lajoie, G.A. Litchfield, D.W., Brandl, C.J., Shaw, G.S., and Shilton, B.H. (2011). Discovery and characterization of a nonphosphorylated cyclic peptide inhibitor of the peptidylprolyl isomerase, Pin1. J. Med. Chem. 54, 3854-3865.

Farrell, A.S., and Sears, R.C. (2014). MYC degradation. Cold Spring Harb. Perspect. Med. 4, http://dx.doi.org/10.1101/cshperspect.a014365.

Farrell, A.S., Pelz, C., Wang, X., Daniel, C.J., Wang, Z., Su, Y., Janghorban, M., Zhang, X., Morgan, C., Impey, S., et al. (2013). Pin1 regulates the dynamics of c-Myc DNA binding to facilitate target gene regulation and oncogenesis. Mol. Cell. Biol. 33, 2930-2949.

Forman-Kay, J.D., and Mittag, T. (2013). From sequence and forces to structure, function, and evolution of intrinsically disordered proteins. Structure 21 1492-1499.

Fuxreiter, M., and Tompa, P. (2012). Fuzzy complexes: a more stochastic view of protein function. Adv. Exp. Med. Biol. 725, 1-14.

Guo, J., Pang, X, and Zhou, H. -X. (2015). Two pathways mediate interdomain allosteric regulation in pin1. Structure 23, 237-247.

Hann, S.R. (2006). Role of post-translational modifications in regulating c-Myc proteolysis, transcriptional activity and biological function. Semin. Cancer Biol. 16, 288-302.

Hariharan, N., and Sussman, M.A. (2014). Pin1: a molecular orchestrator in the heart. Trends Cardiovasc. Med. 24, 256-262.

Hydbring, P., Bahram, F., Su, Y., Tronnersjö, S., Högstrand, K., von der Lehr, N., Sharifi, H.R., Lilischkis, R., Hein, N., Wu, S., et al. (2010). Phosphorylation by Cdk2 is required for Myc to repress Ras-induced senescence in cotransformation. Proc. Natl. Acad. Sci. USA 107, 58-63.

Jacobs, D.M., Saxena, K., Vogtherr, M., Bernado, P., Pons, M., and Fiebic, K.M. (2003). Peptide binding induces large scale changes in inter-domain mobility in human Pin1. J. Biol. Chem. 278, 26174-26182.

Lee, T.H., Chen, C.-H., Suizu, F., Huang, P., Schiene-Fischer, C., Daum, S. Zhang, Y.J., Goate, A., Chen, R.-H., Zhou, X.Z., et al. (2011). Death-associated protein kinase 1 phosphorylates Pin1 and inhibits its prolyl isomerase activity and cellular function. Mol. Cell 42, 147-159.

Legouy, E., DePinho, R., Zimmerman, K., Collum, R., Yancopoulos, G. Mitsock, L., Kriz, R., and Alt, F.W. (1987). Structure and expression of the murine L-myc gene. EMBO J. 6, 3359-3366.

Lin, C.Y., Lovén, J., Rahl, P.B., Paranal, R.M., Burge, C.B., Bradner, J.E., Lee, T.I., and Young. R.A. (2012). Transcriptional amplification in tumor cells with elevated c-Myc. Cell 151, 56-67.

Liou, Y.-C., Zhou, X.Z., and Lu, K.P. (2011). Prolyl isomerase Pin1 as a moleclar switch to determine the fate of phosphoproteins. Trends Biochem. Sci. 36 $501-514$

Lu, K.P., and Zhou, X.Z. (2007). The prolyl isomerase PIN1: a pivotal new twist in phosphorylation signalling and disease. Nat. Rev. Mol. Cell Biol. 8, 904-916.
Lu, P.J., Zhou, X.Z., Shen, M., and Lu, K.P. (1999). Function of WW domains as phosphoserine- or phosphothreonine-binding modules. Science 283, 13251328.

Lu, K.P., Liou, Y.C., and Zhou, X.Z. (2002a). Pinning down proline-directed phosphorylation signaling. Trends Cell Biol. 12, 164-172.

Lu, P.-J., Zhou, X.Z., Liou, Y.-C., Noel, J.P., and Lu, K.P. (2002b). Critical role of WW domain phosphorylation in regulating phosphoserine binding activity and Pin1 function. J. Biol. Chem. 277, 2381-2384.

Lu, K.P., Finn, G., Lee, T.H., and Nicholson, L.K. (2007). Prolyl cis-trans isomerization as a molecular timer. Nat. Chem. Biol. 3, 619-629.

Lukhele, S., Bah, A., Lin, H., Sonenberg, N., and Forman-Kay, J.D. (2013). Interaction of the eukaryotic initiation factor $4 \mathrm{E}$ with $4 \mathrm{E}-\mathrm{BP} 2$ at a dynamic bipartite interface. Structure 21, 2186-2196.

Matena, A., Sinnen, C., van den Boom, J., Wilms, C., Dybowski, J.N., Maltaner, R., Mueller, J.W. Link, N.M., Hoffmann, D., and Bayer, P. (2013). Transient phosphorylated peptide ligands. Structure 21, 1769-1777.

Meyer, N., and Penn, L.Z. (2008). Reflecting on 25 years with MYC. Nat. Rev. Cancer 8, 976-990.

Min, S.-H., Lau, A.W., Lee, T.H., Inuzuka, H., Wei, S., Huang, P., Shaik, S., Lee, D.Y., Finn, G., Balastik, M., et al. (2012). Negative regulation of the stability and tumor suppressor function of Fbw7 by the Pin1 prolyl isomerase. Mol. Cell 46, 771-783.

Mittag, T., Orlicky, S., Choy, W.-Y., Tang, X., Lin, H., Sicheri, F., Kay, L.E., Tyers, M., and Forman-Kay, J.D. (2008). Dynamic equilibrium engagement of a polyvalent ligand with a single-site receptor. Proc. Natl. Acad. Sci. USA 105, 17772-17777.

Namanja, A.T., Peng, T., Zintsmaster, J.S., Elson, A.C., Shakour, M.G., and Peng, J.W. (2007). Substrate recognition reduces side-chain flexibility for conserved hydrophobic residues in human Pin1. Structure 15, 313-327.

Namanja, A.T., Wang, X.J., Xu, B., Mercedes-Camacho, A.Y., Wilson, K.A., Etzkorn, F.A., and Peng, J.W. (2011). Stereospecific gating of functional motions in Pin1. Proc. Natl. Acad. Sci. USA 108, 12289-12294.

Nie, Z., Hu, G., Wei, G., Cui, K., Yamane, A., Resch, W., Wang, R., Green, D.R., Tessarollo, L., Casellas, R., et al. (2012). c-Myc is a universal amplifier of expressed genes in lymphocytes and embryonic stem cells. Cell 151, 68-79.

Peng, J.W. (2015). Investigating dynamic interdomain allostery in Pin1. Biophys. Rev. 7, 239-249.

Qian, B., Raman, S., Das, R., Bradley, P., McCoy, A.J., Read, R.J., and Baker, D. (2007). High resolution protein structure prediction and the crystallographic phase problem. Nature 450, 259-264.

Ranganathan, R., Lu, K.P., Hunter, T., and Noel, J.P. (1997). Structural and functional analysis of the mitotic rotamase Pin1 suggests substrate recognition is phosphorylation dependent. Cell 89, 875-886.

Raveh, B., London, N., Zimmerman, L., and Schueler-Furman, O. (2011). Rosetta FlexPepDock ab-initio: simultaneous folding, docking and refinement of peptides onto their receptors. PLoS One 6, e18934.

Salghetti, S.E., Kim, S.Y., and Tansey, W.P. (1999). Destruction of Myc by ubiquitin-mediated proteolysis: cancer-associated and transforming mutations stabilize Myc. EMBO J. 18, 717-726.

Schumann, F.H., Riepl, H., Maurer, T., Gronwald, W., Neidig, K.-P., and Kalbitzer, H.R. (2007). Combined chemical shift changes and amino acid specific chemical shift mapping of protein-protein interactions. J. Biomol. NMR 39, 275-289.

Sears, R.C. (2004). The life cycle of c-myc: from synthesis to degradation. Cell Cycle 3, 1133-1137.

Tu, W.B., Helander, S., Pilstål, R., Hickman, K.A., Lourenco, C., Jurisica, I., Raught, B., Wallner, B., Sunnerhagen, M., and Penn, L.Z. (2015). Myc and its interactors take shape. Biochim. Biophys. Acta 1849, 469-483.

Verdecia, M.A., Bowman, M.E., Lu, K.P., Hunter, T., and Noel, J.P. (2000). Structural basis for phosphoserine-proline recognition by group IV WW domains. Nat. Struct. Biol. 7, 639-643.

2278 Structure 23, 2267-2279, December 1, 2015 @2015 Elsevier Ltd All rights reserved 
Wang, C., Bradley, P., and Baker, D. (2007). Protein-protein docking with backbone flexibility. J. Mol. Biol. 373, 503-519.

Wang, X., Cunningham, M., Zhang, X., Tokarz, S., Laraway, B., Troxell, M., and Sears, R.C. (2011). Phosphorylation regulates c-Myc's oncogenic activity in the mammary gland. Cancer Res. 71, 925-936.

Werner-Allen, J.W., Lee, C.-J., Liu, P., Nicely, N.I., Wang, S., Greenleaf, A.L. and Zhou, P. (2011). cis-Proline-mediated Ser(P)5 dephosphorylation by the RNA polymerase II C-terminal domain phosphatase Ssu72. J. Biol. Chem. 286, 5717-5726.

Wilson, K.A., Bouchard, J.J., and Peng, J.W. (2013). Interdomain interactions support interdomain communication in human Pin1. Biochemistry 52, 69686981.

Wintjens, R., Wieruszeski, J.M., Drobecq, H., Rousselot-Pailley, P., Buée, L., Lippens, G., and Landrieu, I. (2001). ${ }^{1}$ H NMR study on the binding of Pin 1
Trp-Trp domain with phosphothreonine peptides. J. Biol. Chem. 276, 25150-25156.

Yeh, E., Cunningham, M., Arnold, H., Chasse, D., Monteith, T, Ivaldi, G., Hahn, W.C., Stukenberg, P.T., Shenolikar, S., Uchida, T., et al. (2004). A signalling pathway controlling c-Myc degradation that impacts oncogenic transformation of human cells. Nat. Cell Biol. 6, 308-318.

Zhang, M., Wang, X.J., Chen, X., Bowman, M.E., Luo, Y., Noel, J.P., Ellington, A.D. Etzkorn, F.A, and Zhang, Y. (2012a) Structural and kinetic analysis of prolyl-isomerization/phosphorylation cross-talk in the CTD code. ACS Chem. Biol. 7, 1462-1470.

Zhang, X., Farrell, A.S., Daniel, C.J., Arnold, H., Scanlan, C., Laraway, B.J., Janghorban, M., Lum, L., Chen, D., Troxell, M., et al. (2012b). Mechanistic insight into Myc stabilization in breast cancer involving aberrant Axin1 expression. Proc. Natl. Acad. Sci. USA 109, 2790-2795. 
Structure, Volume 23

\section{Supplemental Information}

\section{Pre-Anchoring of Pin1 to Unphosphorylated c-Myc}

\section{in a Fuzzy Complex Regulates c-Myc Activity}

Sara Helander, Meri Montecchio, Robert Pilstål, Yulong Su, Jacob Kuruvilla, Malin Elvén, Javed M.E. Ziauddin, Madhanagopal Anandapadamanaban, Susana Cristobal, Patrik Lundström, Rosalie C. Sears, Björn Wallner, and Maria Sunnerhagen 


\section{Supplemental Figures and Legends}

Figure S1, Related to Figure 1:

A

MPLNVSFTNR NYDLDYDSVO PYFYCDEEEN FYQQQQQSEL QPPAPSEDIW KKFELLPTPP LSPSRRSGLC SPSYVAVTPF SLRGDNDG

B

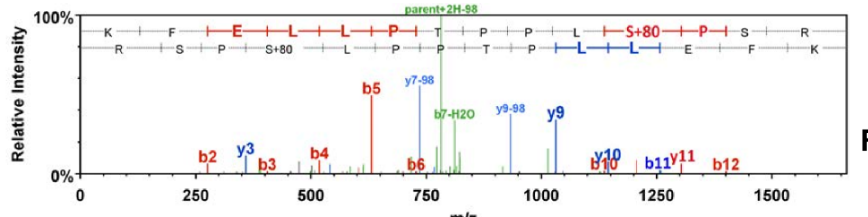

C

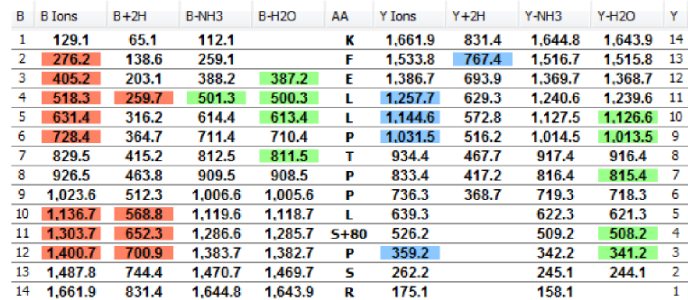

D

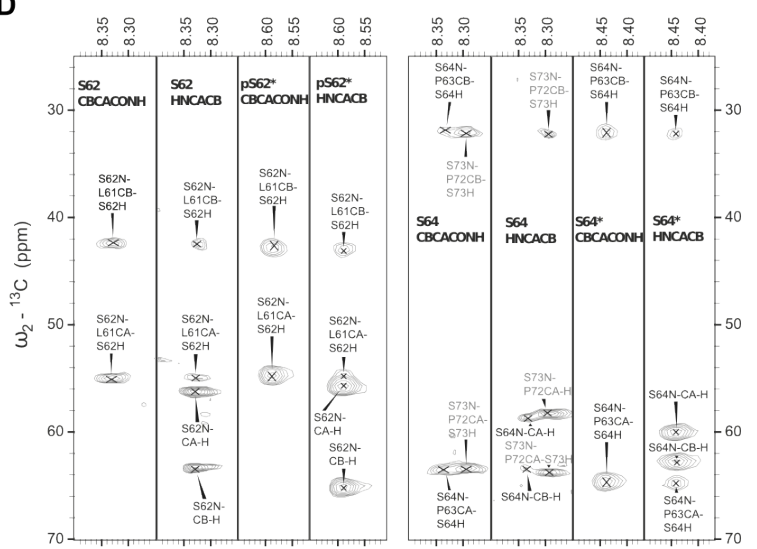

$\omega_{3}-{ }^{1} \mathrm{H}(\mathrm{ppm})$

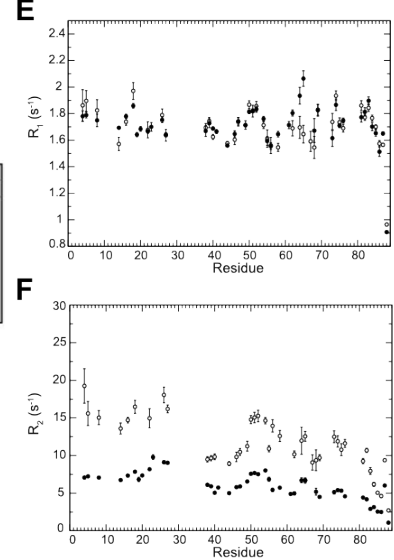

G
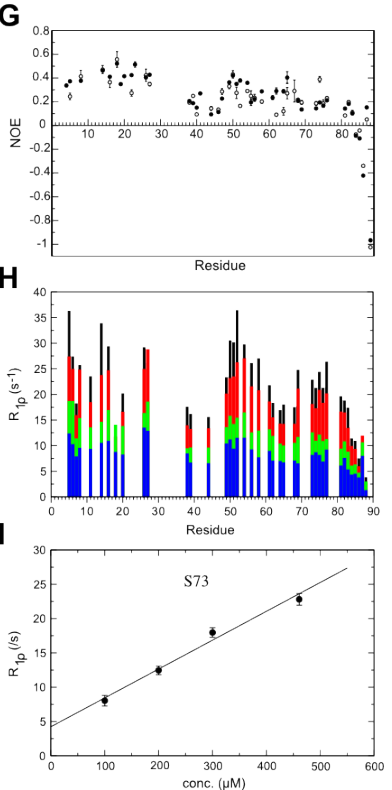

Experimental evaluation of phosphorylation of c-Myc $\mathbf{c}_{1-88}$. (A) Peptides identified by MS/MS (blue) contribute to $9 \%$ of the c-Myc1-88 sequence; Ser62 in red. (B) MS/MS spectra of the peptide fragment FELLPTPPLSPSR. (C) The corresponding lists of fragment ions (M/Z values) identified in Mascot search shows only Ser62 phosphorylation by CDK2; multiple phosphorylations including Ser64 and Thr58 were observed with Gsk3 and Erk treatment (data not shown). (D) Amide-correlated peak strips for Ser62 and Ser64 CBCACONH and HNCACB spectra (*: phosphorylated) show a phosphodependent ${ }^{13} \mathrm{C} \beta$ shift of +1.8 ppm at Ser62 as expected (Bienkiewicz and Lumb 1999). (E-G) Relaxation parameters of apo c-Myc $1-88$ (open circles) and Ser62 phosphorylated c-Myc $\mathrm{c}_{1-88}$ (filled circles); (E) $\mathrm{R}_{1}$ relaxation rates, $(\mathbf{F}) \mathrm{R}_{2}$ relaxation rates and $(\mathbf{G}){ }^{1} \mathrm{H}^{-15} \mathrm{~N}-\mathrm{NOE}$. $(\mathbf{H}) \mathrm{R}_{1 \mathrm{p}}$ relaxation rates for various concentrations of c-Myc $\mathrm{C}_{1-88} ; 460 \mu \mathrm{M}$ (black), $300 \mu \mathrm{M}$ (red) $200 \mu \mathrm{M}$ (green) and $100 \mu \mathrm{M}$ (blue). As exemplified by residue $\operatorname{Ser} 73$ (I) $\mathrm{R}_{1 \rho}$ relaxation rates decrease with decreasing concentrations of c-Myc $\mathrm{c}_{1-88}$. 
Figure S2, Related to Figure 2:

A

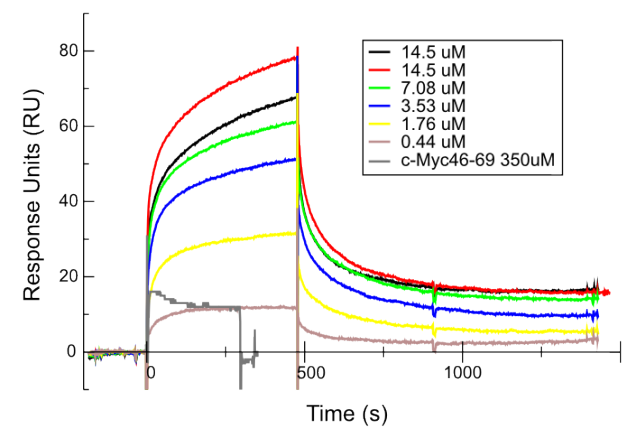

C

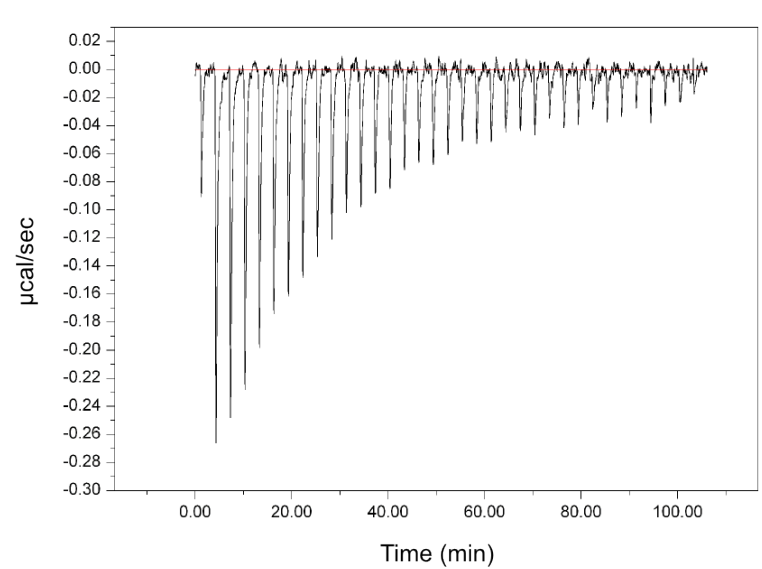

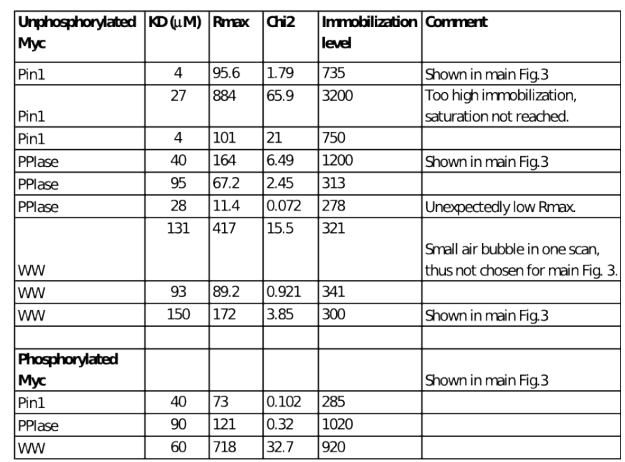

D

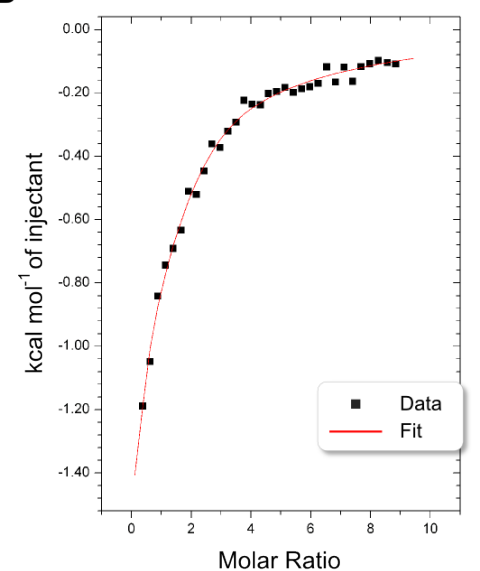

E

\begin{tabular}{|c|c|c|c|}
\hline \multicolumn{2}{|c|}{3 sites } & \multicolumn{2}{|c|}{4 sites } \\
\hline $\mathrm{K}_{\mathrm{d}}[\mu \mathrm{M}]$ & $\Delta \mathrm{H}\left[\mathrm{kcal} \mathrm{mol}^{-1}\right]$ & $\mathrm{K}_{\mathrm{d}}[\mu \mathrm{M}]$ & $\Delta \mathrm{H}\left[\mathrm{kcal} \mathrm{mol}^{-1}\right]$ \\
\hline $19.5=$ & $-3 \pm 0.2$ & $25 \pm 0.9$ & $-3 \pm 0.1$ \\
\hline $10 \pm 3.2$ & $-1.4 \pm 0.3$ & $10 \pm 0.9$ & $-1.4 \pm 0.2$ \\
\hline $110 \pm 7.8$ & $-3 \pm$ & $\begin{array}{l}120 \pm 5.2 \\
178 \pm 20\end{array}$ & $\begin{aligned}-3 & \pm 0.14 \\
-4.2 & \pm 0.5\end{aligned}$ \\
\hline
\end{tabular}

Pin1-Myc $\mathbf{1}_{-88}$ binding by SPR and ITC. (A) SPR sensorgrams for Pin1 binding to immobilized c-Myc $\mathrm{c}_{1-88}$ (in color). The grey sensorgram shows the response when Pin1 is immobilized and c-Myc $\mathrm{C}_{46-69}$ injected over the surface at a concentration of 350 $\mu \mathrm{M}$; this was the maximum response obtained, which together with the very rapid on and off rates indicated no specific binding. (B) Table showing the experimental parameters for the performed complete SPR experiments, excluding scouting and trial runs. (C) Raw data for the ITC titration of Pin1 showing the calorimetric response from successive injections to pS62-c-Myc 1-88. $_{\text {. }}$ (D) Integrated heat changes and fitting of the binding profile to a three-state model as a red solid line, with squares representing the heat per injection (first dummy injection is removed). Curves are best fits to the data to obtain reduced $\chi^{2}$ value. (E) ITC

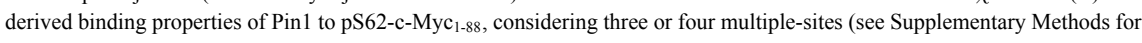
details on ITC measurement and evaluation). 
Figure S3, Related to Figure 3:
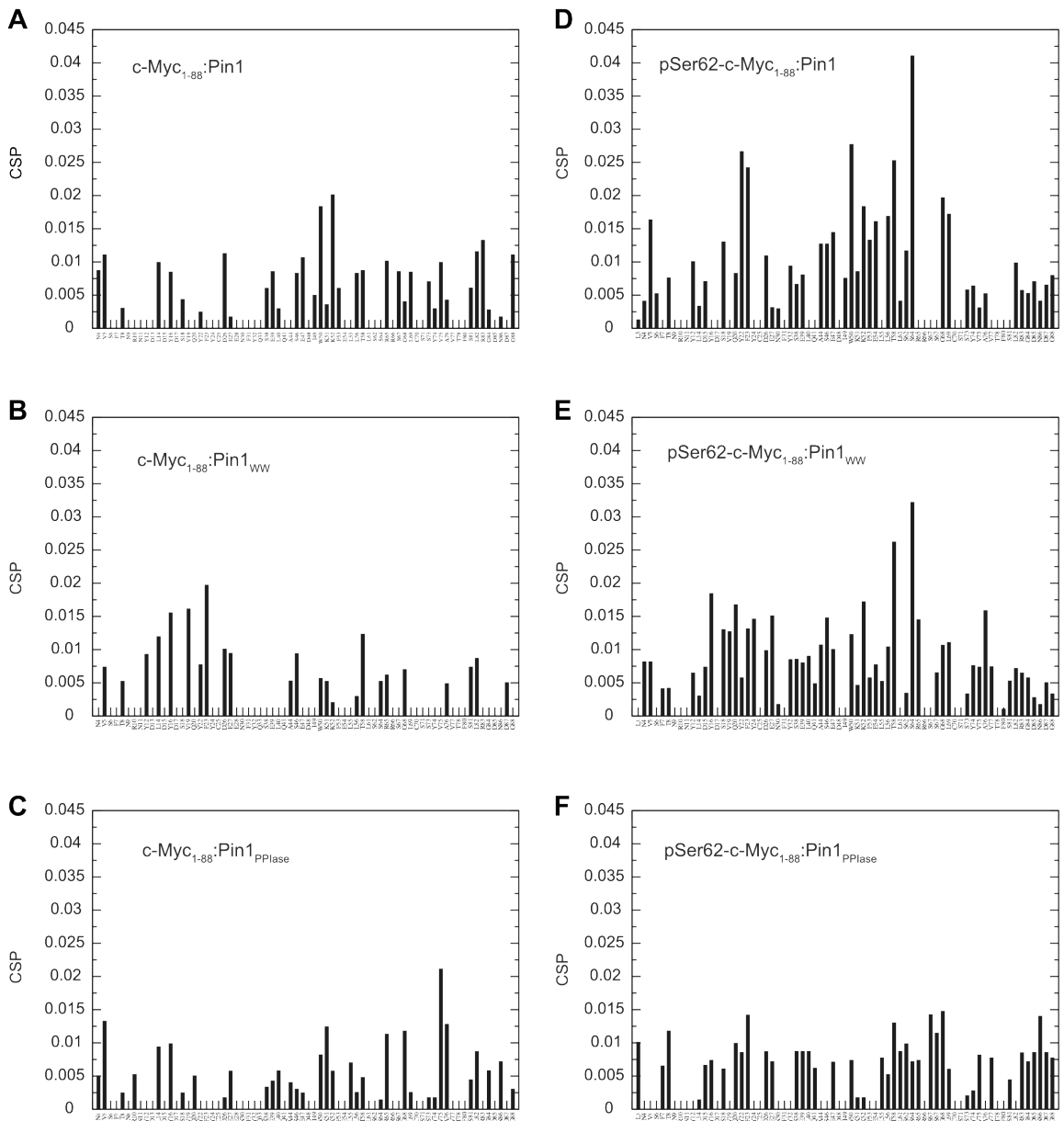

Chemical shift pertubations (CSPs) for unphosphorylated and phosphorylated c-Myc $\mathrm{c}_{1-88}$ on Pin1 binding. Histograms show CSPs for $\mathrm{c}-\mathrm{Myc}_{1-88}$ in complex with (A) 0.8 eq Pin1, (B) 0.75 eq Pin $1_{\mathrm{ww}}$ or (C) 0.7 eq Pin $1_{\text {PPlase, }}$, and for pSer62-c$\mathrm{Myc}_{1-88}$ in complex with 0.7 eq Pin 1 (D), Pin1 $1_{\mathrm{ww}}(\mathbf{E})$ or Pin $1_{\text {PPlase }}(\mathbf{F})$. At higher content of Pin1, CSPs can no longer reliably

be measured for many resonances due to extensive line broadening. 
Figure S4, Related to Figure 5:
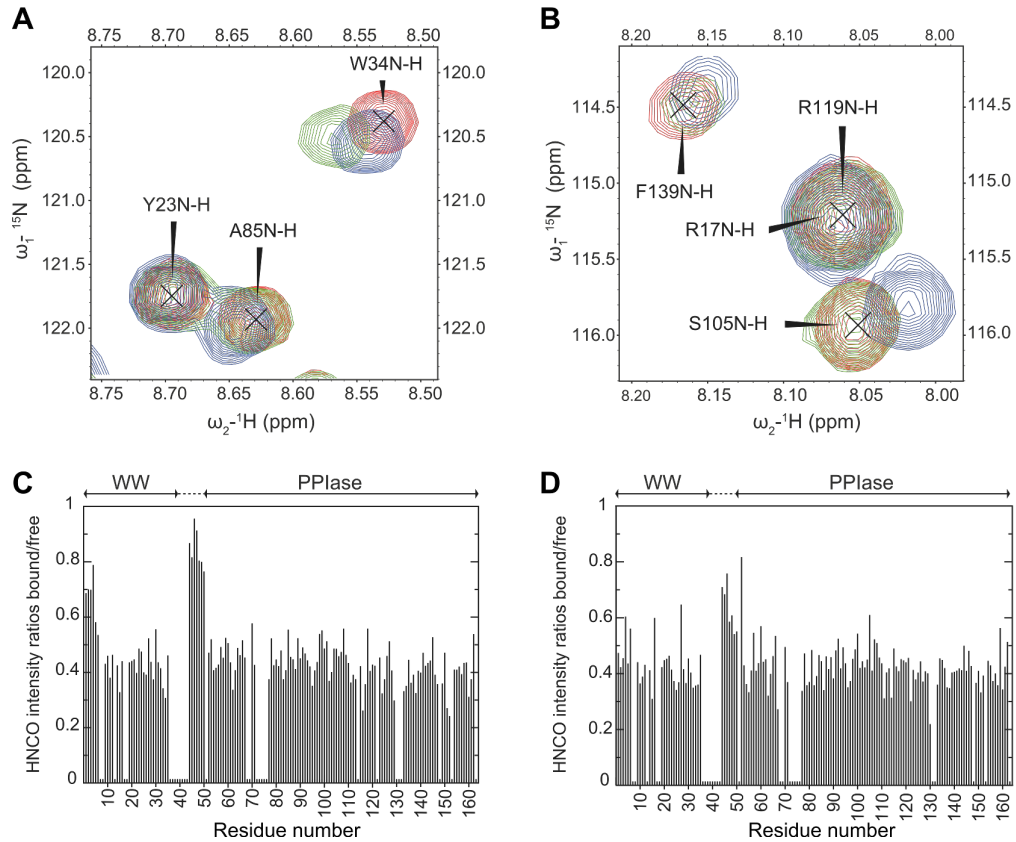

Details of Pin1 chemical shift perturbations and HNCO peak ratios. Magnification of some of the most dramatic chemical shifts upon binding (A, B) (red: Pin1; green: Pin1 with c-Myc $1-88$ 1:1; blue: Pin1 with pS62-c-Myc $\mathrm{c}_{1-88}$ 1:1). HNCO peak ratios for the complex c-Myc $1_{1-88}$ :Pin 1 (C) and for pS62-c-Myc $1-88$ :Pin1 (D). HNCO peak intensity ratios for both Pin $1_{\mathrm{ww}}$ and Pin $1_{\text {PPlase }}$ average to 0.4 . These results are in fact surprising since the SPR data yielded a $\mathrm{K}_{\mathrm{D}}$ of $4 \mu \mathrm{M}$ for $\mathrm{c}-\mathrm{Myc}_{1-88}$ :Pin 1 and $40 \mu \mathrm{M}$ for $\mathrm{pSer} 62-\mathrm{c}-\mathrm{Myc}_{1-88}$ :Pin1. At the protein concentrations used here, this means that the complexes are populated to $86 \%$ and $63 \%$, respectively, and thus that the average peak intensity ratio for pSer62-c-Myc $1-88$ :Pin1 is expected to be higher based on molecular weight considerations only. That the average ratios in fact are equal indicates that tumbling of Pin1 is affected differently upon binding of $\mathrm{c}-\mathrm{Myc}_{1-88}$ and pSer62-c-Myc $\mathrm{c}_{-88}$; this is in agreement with differential effects on Pin $1 \tau_{\mathrm{c}}$ on binding to different substrates (Jacobs et al. 2003). The average ratios for the linker (0.8/0.6 for c-Myc $\mathrm{C}_{1-88} \mathrm{vs.}$ pSer62-c-Myc $\left.\mathrm{C}_{-88}\right)$ differ in the expected direction, and the higher average values for the linker region in both cases suggests that it maintains an independent level of dynamics relative to Pin $1_{\mathrm{Ww}}$ and Pin $1_{\mathrm{PPlase}}$. 
Figure S5, Related to Figure 6:

A
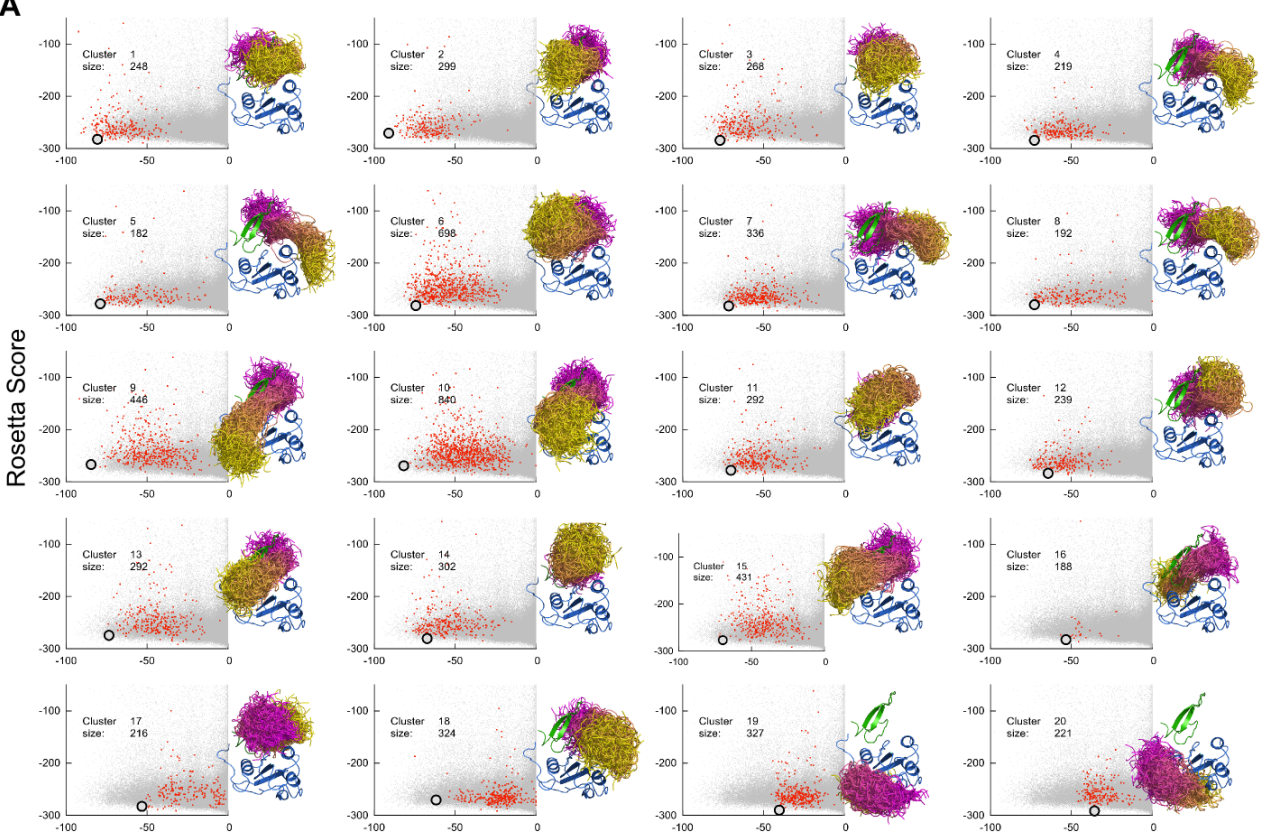

Constraint Score

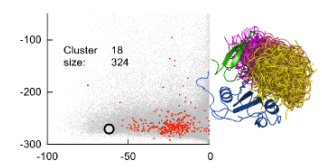

Constraint Score

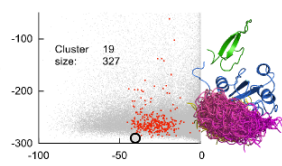

Constraint Score

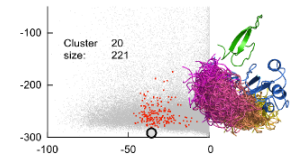

Constraint Score

B

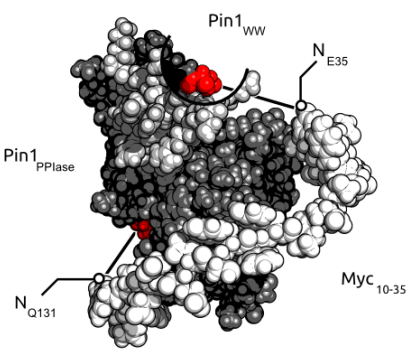

C

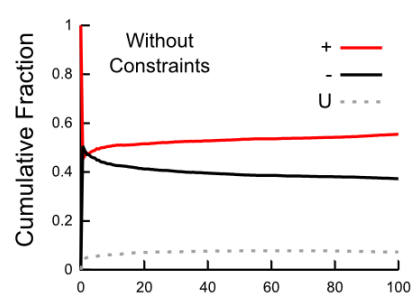

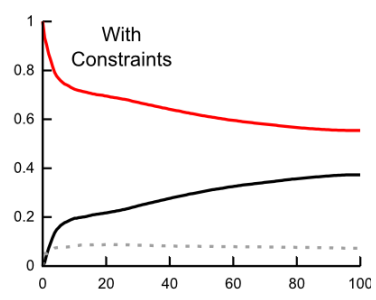

$\%$ of models

Description of the Pin1 bound ensemble of unphosphorylated c-Myc $10-35$. (A) Detailed presentation of the 20 largest clusters for $\mathrm{c}-\mathrm{Myc}_{10-35}$ bound to Pin1. The clusters are reversely ranked by the sum of Rosetta and Constraint scores of their lowestscore representatives. Each cluster is represented by an energy distribution plot, including cluster rank and size, together with a PyMOL render displaying the included c- $\mathrm{Myc}_{10-35}$ conformations. Rosetta standard and experimental constraint score is on the vertical and horizontal axis respectively. Indicated are models included in the cluster (red), all models (grey) and the lowestscore representative (circle). Note that clusters 16-20 do not satisfy the experimental data at the same level as the first 15 since they have a higher constraint score term. For this reason they were not included in the ensemble describing the bound state. (B) The integer difference D between the residue number of the closest c-Myc $\mathrm{M}_{10-35}$ residue to Pin1 residues Q131 and E35 $\left(\mathrm{D}=\mathrm{N}_{\mathrm{Q} 131}-\mathrm{N}_{\mathrm{E} 35}\right)$ was used to measure the direction. $\mathrm{c}-\mathrm{Myc}_{10-35}$ models with their N-terminal part closer to $\mathrm{E} 35$ than Q131 have a positive direction $(\mathrm{D}>0)$, while models with their $\mathrm{C}$-terminal part closer to $\mathrm{E} 35$ than $\mathrm{Q} 131$ have a negative direction $(\mathrm{D}<0)$. Pin1 residues E35 and Q131 are highlighted in red, and an illustrative c-Myc $\mathrm{c}_{10-35}$ is displayed in white. A cutout has been made in the Pin $1_{\mathrm{WW}}$ domain in order to display E35, which would otherwise be obscured from view. Examples of closest c-Myc $\mathrm{C}_{10-35}$ residues are labeled $\mathrm{N}_{\mathrm{Q} 131}$ and $\mathrm{N}_{\mathrm{E} 35}$. In $(\mathbf{C})$ the cumulative fractions of models, owning one or the other or no direction $(\mathrm{D}=0)$, are continuously (cumulative) plotted for different top percentages of models, sorted with and without the constraint score term. 
Figure S6, Related to Figure 6:
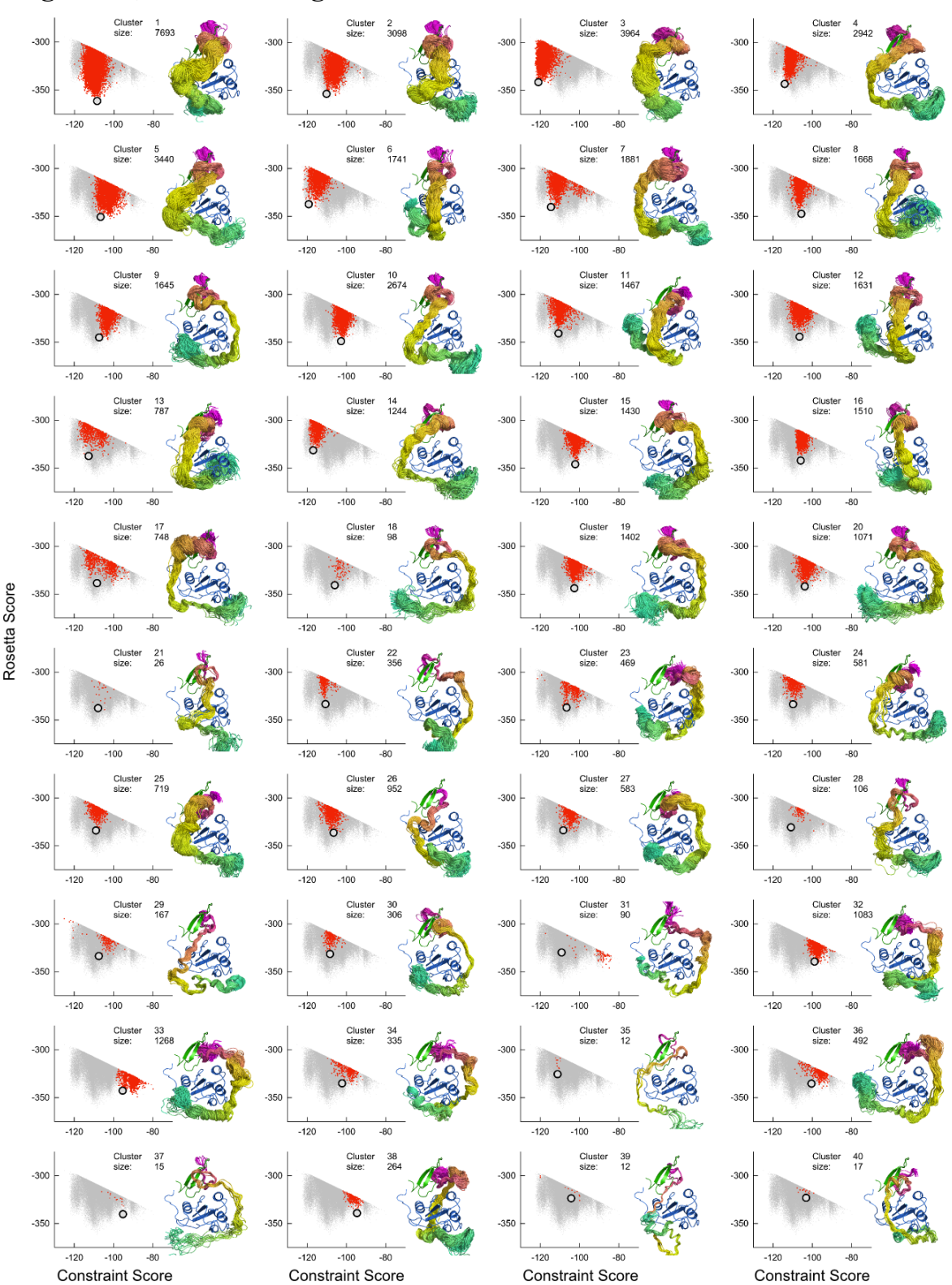

Description of the Pin1 bound ensemble of unphosphorylated $\mathbf{c - M y c} \mathbf{M}_{10-70}$. Detailed presentation of the 40 largest clusters for $\mathrm{c}-\mathrm{Myc}_{10-70}$ bound to Pin1. The clusters are reversely ranked by the sum of Rosetta and Constraint scores of their lowestscore representatives. Each cluster is represented by an energy distribution plot, including cluster rank and size, together with a PyMOL render displaying the included c-Myc ${ }_{10-70}$ conformations. Rosetta standard and experimental constraint score is on the vertical and horizontal axis respectively. Indicated are models included in the cluster (red), all models (grey) and the lowestscore representative (circle). Clusters 1 through 17 are all significant in size ( $>10 \%$ of the largest cluster) and were thus jointly chosen as a representative set for visualization of the ensemble in Figure 6. 
Figure S7, Related to Figure 7:

A
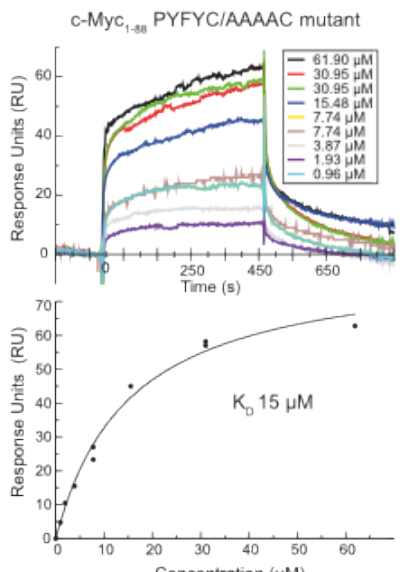

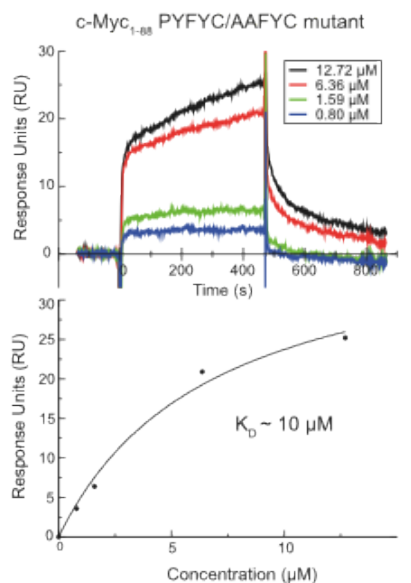

C

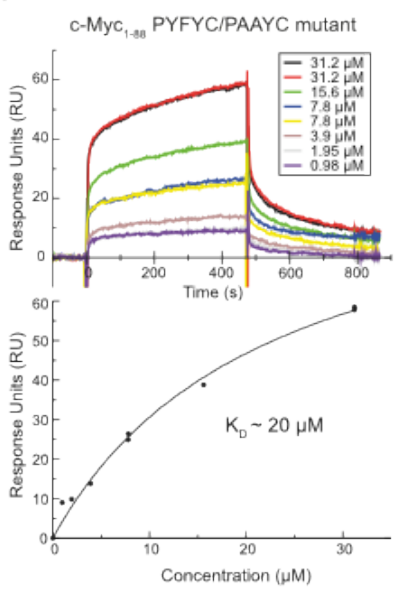

D

\begin{tabular}{|l|l|l|l|l|l|}
\hline Unphosphorylated Myc & $\mathbf{K}_{\mathrm{D}}(\boldsymbol{\mu} \mathbf{M})$ & Rmax & Chi2 & $\begin{array}{l}\text { Immobilization } \\
\text { level }\end{array}$ & Comment \\
\hline Myc mutants & & & & & \\
\hline 20-PYFYC/AAAAC-24 & 15 & 83.2 & 8.1 & 376 & \\
\hline 20-PYFYC/AAFYC-24 & 10 & 66.5 & 4.33 & 400 & \\
\hline 20-PYFYC/PAAYC-24 & 20 & 99.1 & 5.41 & 469 & Saturation not reached. \\
\hline
\end{tabular}

E

Pintide, reverse ELFËSYFW

Cyclic peptide CTGIPWLYC

C-MYC $_{11-70} \quad$ NYDLDYDSVQPYFYCDEEENFYQQQQQSELQPPAPSEDIWKKFELLPTPPLSPSRRSGLC

AP1/Jun $_{11.70}$ DDALNASFLPSESGPYGYSNPKILKQSMTLNLADPVGSLKPHLRAKNSDLLTSPDVGLLK

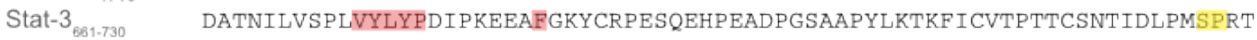
RAR-alpha ${ }_{11-80}$ PGGGHLNGYPVPPYAFFFPPMLGGLSPPGALTTLQHQLPVSGYSTPSPATIETQSSSSEEIVPSPPSPPP C-FoS $_{341-390}$ TPSCTAYTSSFVFTYPEADSFPSCAAAHRKGSSSNEPSSDSLSSPTLLAL

Biophysical and bioinformatics evaluation of mutant c-Myc binding to Pin1. Surface plasmon resonance (SPR) measurements shown in (A-C) and described in table (D) concern the binding of full length Pin1 to c-Myc $1-88$ (A) 20-PYFYC/AAAAC (B) 20-PYFYC/AAFYC or (C) 20-PYFYC/PAAYC mutants. All these mutant constructs are able to bind Pin1, but with weaker affinity than wt. For all measurements $\mathrm{c}-\mathrm{Myc}_{1-88}$ was immobilized and Pin1 was injected over the surface in serial dilutions and the evaluation using a 1:1 Langmuir model is detailed in the table in (D). (E) By manually inspecting Pin1 targets with a defined pSer/pThr site (Liou, Zhou, and Lu 2011) we found that a sequence motif with similarities to the Pin1-binding, N-terminal motif in c-Myc is present also in several other pro-proliferative transcriptional regulators. The Pin1 binding motif in c-Myc and its tentative similar binding sites in the two Pin1 binding peptides (Yaffe et al. 1997; Duncan et al. 2011) as well as in AP1/Jun, Stat-3, RAR-alpha and c-Fos are labeled red. The identified Pin1-targeted phosphorylation sites for c-Myc, AP1/Jun, Stat-3, RAR-alpha and c-Fos (Hann 2006; Wulf et al. 2001; Lufei et al. 2007; Brondani et al. 2005; Monje et al. 2005) are highlighted in yellow. 
Movie S1, Related to Figure 6: Visualization of c-Myc ensembles bound to Pin1. The movie shows the ensembles of c-Myc bound to Pin1 as in Figure 6. The entire ensemble and the lowest energy representative from each cluster is displayed sequentially while the whole model is being rotated through select views. The movie starts by showing the bound ensemble of $\mathrm{c}-\mathrm{Myc}_{10-35}$, as in Figure 6d,e, and then similarly shows the bound ensemble of c-Myc $\mathrm{M}_{10-70}$, as in Figure 6e,f. 


\section{Supplemental Experimental Procedures}

Bioinformatic sequence evaluation

A Profile hidden Markov Model (HMM) $\operatorname{logo}$ was constructed for $\mathrm{c}-\mathrm{Myc}_{1-88}$, where each residue position is visualized with a stack of single letter amino acid names: high stacks with large letters signify conservation (Schuster-Böckler et al., 2004). To construct the logo, seed sequences for Pfam entry PF01056 were downloaded from uniprot.org and realigned with Muscle (Edgar, 2004a; 2004b; Finn et al., 2014). A Hidden Markov Model (HMM) was then constructed from all columns spanned by c$\mathrm{Myc}_{1-88}$ in the resulting alignment (using the HMMER software suite program hmmbuild, see http://www.hmmer.org/), which was then employed to search for homologs in Uniref90 (Suzek et al., 2007). Finally a HMM was built from the resulting alignment with the consensus columns pertaining to the c-Myc (UniProt ID:P01106) sequence and submitted to the Skylign (Wheeler et al., 2014) webserver to generate the logo. The HMM only represents residues 1-74 out of $\mathrm{Myc}_{1-88}$ studied experimentally in this work, since the most C-terminal part of $\mathrm{Myc}_{1-88}$ was not sufficiently prevalent in the database (Tu et al., 2015).

\section{Protein expression and purification}

Vectors for the human Pin1 constructs were kindly received from Prof. Bayer, University of Duisburg-Essen and contained full-length (1-163) Pin1 in pET-28a, Pin $1_{\text {ww }}$ (a.a. 6-39) in pET-41a and Pin $1_{\text {PPlase }}$ (a.a. 50-163) in pET-42_mod_TEV (Bayer et al., 2003). For protein production, the appropriate vector was transformed into BL21(DE3) CodonPlus cells and incubated overnight at $37^{\circ} \mathrm{C}$. The overnight cultures were diluted into fresh Terrific Broth media, containing the appropriate antibiotics for each construct, or in M9 minimal media with ${ }^{13} \mathrm{C}$-glucose and/or ${ }^{15} \mathrm{NH}_{4} \mathrm{Cl}$ (Sigma-Aldrich and Cambridge Isotope Laboratories) for singly or doubly labeled samples. The cultures were grown until $\mathrm{OD}_{600}=1.5$ in TB or 0.7 in $\mathrm{M} 9$ and induced with $1 \mathrm{mM}$ isopropyl- $\beta$-D-1-thiogalactopyranoside (IPTG) and expressed overnight at $20^{\circ} \mathrm{C}$. Cells were harvested and resuspended in $50 \mathrm{mM} \mathrm{NaH}_{2} \mathrm{PO}_{4}, 300 \mathrm{mM}$ $\mathrm{NaCl}, 10 \mathrm{mM}$ imidazole, Complete ${ }^{\mathrm{TM}}$ EDTA-free, protease inhibitor (Roche Applied Science), pH 8.0. Following sonication, the lysates were centrifuged at $18000 \mathrm{~g}, 4^{\circ} \mathrm{C}$ for $30 \mathrm{~min}$. Except for the use of $\mathrm{Co}^{2+}$ beads (TALON® Metal Affinity Resin, 
Clontech), Pin1, Pin $1_{\mathrm{Ww}}$ and Pin $1_{\text {PPlase }}$ proteins were then purified according to the previous published protocol (Bayer et al., 2003). The $\mathrm{c}-\mathrm{Myc}_{1-88}$ protein and the three different $\mathrm{c}-\mathrm{Myc}_{1-88}$ mutants (PYFYC/AAAAC, AAFYC or PAAYC) were expressed and purified as described earlier (Andresen et al., 2012), for NMR with appropriately labelled Spectra-9 rich growth media (Cambridge Isotope Laboratories). Protein purity was analyzed by SDS-PAGE and MALDI.

Phosphorylation of $c-M y c_{1-88}$

The phosphorylation of $\mathrm{c}-\mathrm{Myc}_{1-88}$, was performed at a protein concentration of $40 \mu \mathrm{M}$ and a final concentration of $0.0001 \mu \mathrm{g} / \mu \mathrm{l} \mathrm{CDK} 2 / \mathrm{Cyclin} \mathrm{A} 2$ kinase (Promega). The reaction was allowed to proceed at $20^{\circ} \mathrm{C}$ for $20 \mathrm{~h}$. Prior to the phosphorylation reaction, $\mathrm{c}-\mathrm{Myc}_{1-88}$ was dialyzed against phosphorylation buffer, comprising $20 \mathrm{mM}$

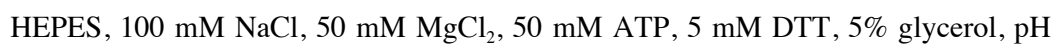
7.0 .

Liquid chromatography mass spectrometry (LC-MS/MS)

The purified protein (unphosphorylated control and phosphorylated samples) was resuspended in $50 \mathrm{mM}$ ammonium bicarbonate, reduced for 30 minutes with $2 \mathrm{mM}$ DTT and alkylated by $6 \mathrm{mM}$ iodoacetamide for $30 \mathrm{~min}$ at room temperature. The endopeptidase trypsin (Promega) was added to the protein, $0.03 \mathrm{mg}$ trypsin per milligram protein for effective digestion, which was performed at $37^{\circ} \mathrm{C}$ for $15 \mathrm{~h}$. The peptides fragments were centrifuged, desalted using stage tips and vacuum dried to be analyzed by LC-MS/MS. The tryptic peptides were then separated using a nano-flow reversed phase HPLC (Bruker Daltronics, Germany) using a C18 reverse phase column (100mm x 75mm, particle size: $5 \mathrm{~mm}$ ) (Buffer A: $0.1 \%$ formic acid in water. Buffer B: $0.1 \%$ formic acid in acetonitrile). A linear gradient at a constant flow rate of $300 \mathrm{nl} / \mathrm{min}$ from 0 to $100 \%$ buffer B was employed to achieve a good separation. Peptides were analyzed on an HCTultra PTM Discovery System (Bruker Daltronics, Germany) online ion-trap Mass Spectrometer in the positive mode. Fragmentation was achieved by the techniques collision induced ionization (CID) and electron transfer dissociation (ETD) in this online tandem MS setup. The raw data was processed into peak lists as Mascot generic format (MGF files) using Data Analysis 3.4 (Bruker Daltronics, Germany). These processed files were uploaded and searched 
for Homo sapiens protein database from Swissprot. The searches were conducted with an allowed mass error of 1.2 Da for MS data and 0.8 Da for fragments in case of CID fragmentation while a mass error of 0.8 Da were allowed for MS data and 0.6 Da for fragments in ETD fragmentation. Missed cleavages of 2 were allowed with different charge states while fixed modification was cystine carbamidomethylation and phosphorylations on Ser, Thr and Tyr were considered under variable modifications. For both techniques, MASCOT score above 25 and minimum of 2 valid peptide hits confirmed the identifications.

\section{Surface plasmon resonance (SPR) experiments}

SPR measurements were performed at $25^{\circ} \mathrm{C}$ using a Biacore 300 instrument. The c$\mathrm{Myc}_{1-88}$ protein and the different mutants was diluted in acetate buffer $\mathrm{pH} 4.5$ and immobilized to sensor chip CM5 research grade (GE Healthcare) by amine binding, to an immobilization level of approximately 300-400 RU. Running buffers for Pin1, Pin $1_{\mathrm{wW}}$ and Pin $1_{\text {PPIase }}$ were $40 \mathrm{mM} \mathrm{HEPES,} 200 \mathrm{mM} \mathrm{NaCl}, 5 \mathrm{mM}$ TCEP, 5\% glycerol, $0.05 \%$ Tween 20, $\mathrm{pH}$ 7.0. Affinity measurements were performed using different concentrations of Pin1, Pin $1_{\mathrm{WW}}$ or Pin $1_{\mathrm{PPI} \text { ase }}$ as described (random order injection) and the surface was regenerated with $3 \mathrm{mM} \mathrm{NaOH}$ (30 second pulse, flow $10 \mu \mathrm{l} / \mathrm{min}$ ). Steady state data was evaluated using the BIAEvaluation 4.1 software (GE Healthcare). All sensorgrams were double reference subtracted. The affinities

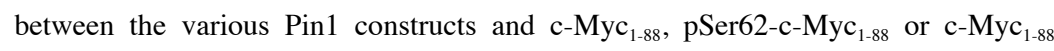
mutants were all evaluated using a 1:1 Langmuir model.

\section{Isothermal Titration Calorimetry (ITC) experiments}

The ITC experiment was performed using VP ITC instrument (Microcal), with the concentration of $20 \mu \mathrm{M}$ c-Myc1-88 in the cell $(1.43 \mathrm{ml})$ and $900 \mu \mathrm{M}$ Pin $1(280 \mu \mathrm{l})$ in the syringe, in Hepes buffer, pH 7 (20 mM HEPES, $100 \mathrm{mM} \mathrm{NaCl}, 0.1 \mathrm{mM}$ TCEP). Resulting binding isotherms were analyzed according to non-linear regression fit using multiple binding site models with three and four sites, in Origin software (OriginLab, Northampton, MA, USA). Due to the overlapping binding processes, the definition of the number of sites and the dissociation constant is not well defined. However, single or dual site models do not fit the data, while multiple binding processes significantly reduce the Chi2 value of the fit, indicating that that numerous 
events come into action in the interplay between Pin1 and the phosphorylated cMyc1-88. Importantly, the control dilution experiment of the Pin1 at a concentration of $1.2 \mathrm{mM}$ to buffer titration shows no presence of change in the heat signal.

NMR spectroscopy

All experiments were performed using a $600 \mathrm{MHz}$ Varian INOVA spectrometer equipped with a cryoprobe. Initial titration experiments for c-Myc $\mathrm{C}_{1-88}$ (unphosphorylated or phosphorylated) and the various constructs of Pin1 were performed with initial c- $\mathrm{Myc}_{1-88}$ concentrations ranging between $150 \mu \mathrm{M}$ and $110 \mu \mathrm{M}$. ${ }^{15} \mathrm{~N}-\mathrm{HSQC}$ spectra were recorded at $0.1,0.3,0.7,1.0,1.5$ and 2.0 equivalents of Pin1, Pin $1_{\mathrm{wW}}$ or Pin $1_{\text {PPIase }}$. HNCO intensity ratios were measured using 1 mole equivalent of

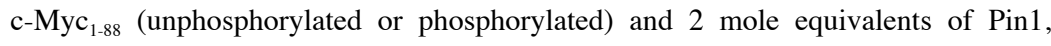
Pin $1_{\mathrm{WW}}$ or Pin $1_{\mathrm{PPI} \text { ase }}$, at Myc concentrations ranging from 81 to $115 \mu \mathrm{M}$ and at $15^{\circ} \mathrm{C}$. A tenfold excess of Pin1 with respect to Myc did not lead to recovery of line-broadened resonances, likely due to continued chemical exchange in the bound state(Bozoky et al., 2013). The low concentrations were possible due to the excellent signal-to-noise for the intrinsically disordered Myc. Both free and bound $\mathrm{c}-\mathrm{Myc}_{1-88}$ proteins were analysed at identical experimental conditions and the same concentration of c-Myc $\mathrm{M}_{1-88}$ All experiments were run in $20 \mathrm{mM}$ HEPES, $100 \mathrm{mM} \mathrm{NaCl}, 5 \mathrm{mM}$ DTT and $5 \%$ glycerol, pH 7.0. The c-Myc ${ }_{1-88}$ (apo) samples for each measurement were diluted with buffer and run at the same concentration and conditions as for the complex samples. The ${ }^{15} \mathrm{~N}-\mathrm{HSQC}$ and HNCO experiments for the surface mapping on the full length Pin 1 were performed at $25^{\circ} \mathrm{C}$, in the same buffer used for the Myc experiments, with $180 \mu \mathrm{M}{ }^{15} \mathrm{~N},{ }^{13} \mathrm{C}$ labeled Pin1 and unphosphorylated $\mathrm{c}-\mathrm{Myc}_{1-88}$ or pSer62-c- $\mathrm{Myc}_{1-88}$ present in molar ratio 1:1. The higher concentrations used in these experiments compared to the HNCO titrations were required due to the larger size and higher degree of structuredness of Pin1, resulting in broader line widths and thereby intrinsically lower signal intensity.

Assignment of unphosphorylated and phosphorylated $c-M y c_{1-88}$ Standard pulse sequences for HNCO, HNCA, HNCACB, HN(CO)CA, HN(CA)CO, CBCA(CO)NH, and HACAN (Sattler et al., 1999) were used to record spectra for backbone resonance assignment. The HACAN experiment was particularly useful to 
trace the assignment through the prolines and resulted in additional assignments compared to our previous study. All spectra were recorded at $15^{\circ} \mathrm{C}$, using $150 \mu \mathrm{M} \mathrm{c}$ $\mathrm{Myc}_{1-88}$ in $20 \mathrm{mM}$ HEPES, $100 \mathrm{mM} \mathrm{NaCl}, 5 \mathrm{mM}$ DTT, 5\% glycerol, pH 7.0. Assignments for both $\mathrm{c}-\mathrm{Myc}_{1-88}$ and pSer62-c-Myc $\mathrm{C}_{1-88}$ are being deposited in the BMRB database.

\section{Relaxation experiments}

The ${ }^{15} \mathrm{~N}-\mathrm{R}_{1},{ }^{15} \mathrm{~N}-\mathrm{R}_{10}$ and $\left\{{ }^{1} \mathrm{H}\right\}-{ }^{15} \mathrm{~N}-\mathrm{NOE}$ relaxation experiments for unphosphorylated c- $\mathrm{Myc}_{1-88}$ and phosphorylated $\mathrm{c}-\mathrm{Myc}_{1-88}$ were recorded using a protein concentration of $250 \mu \mathrm{M}$, which was required for sensitivity. The $\mathrm{R}_{1}, \mathrm{R}_{1 \mathrm{Q}}$ and heteronuclear NOE relaxation experiments for $\mathrm{c}-\mathrm{Myc}_{1-88}$ :Pin1 1:2 complex was recorded using the same concentration of $\mathrm{c}-\mathrm{Myc}_{1-88}$, resulting in saturation levels for the $\mathrm{c}-\mathrm{Myc}_{1-88}: \operatorname{Pin} 1$ complex of $98 \%$. For the $\mathrm{R}_{1}$ experiments, 25 data points were recorded, using relaxation delays between 10 and $756 \mathrm{~ms}$. For $\mathrm{R}_{1 \mathrm{e}}$ experiments, 19 data points were recorded with relaxation delays between 6 and $100 \mathrm{~ms}$. The spin lock field strength was $1754 \mathrm{~Hz}$ for c-Myc $\mathrm{My}_{1-88}$ (apo) and $1760 \mathrm{~Hz}$ for c-Myc $\mathrm{M}_{1-88}$ (complex) and the carrier was positioned at $119.0 \mathrm{ppm}$ during spin lock. The heteronuclear NOE experiment was recorded by including or not including a $5 \mathrm{~s}$ period of $120^{\circ}{ }^{1} \mathrm{H}$ saturation pulses, separated by $5 \mathrm{~ms}$. The total recovery delay was $12 \mathrm{~s}$. Uncertainties in peak intensities were estimated from duplicate data points.

\section{Data Analysis}

All spectra were processed with NMRpipe(Delaglio et al., 1995) and further analyzed using SPARKY (T. D. Goddard and D. G. Kneller, SPARKY 3, University of California, San Francisco). The HNCO peak intensities for free c-Myc $\mathrm{M}_{1-88}$ measured in experiences described above were compared with the peak intensities for the various complexes and the ratios between bound and free $\mathrm{c}-\mathrm{Myc}_{1-88}$ were calculated. In the HNCO peak intensity experiment, using affinities estimated from SPR measurements the saturation levels for unphosphorylated $\mathrm{c}-\mathrm{Myc}_{1-88}$ and Pin1, Pin $1_{\mathrm{ww}}$ or Pin $1_{\text {PPIase }}$ were $96 \%, 58 \%$ and $73 \%$, respectively, whereas for phosphorylated c$\mathrm{Myc}_{1-88}$ they were $78 \%, 69 \%$ and $63 \%$. The ${ }^{1} \mathrm{H}$ and ${ }^{15} \mathrm{~N}$ chemical shift difference between apo and complex sample was calculated using the equation $\Delta \delta_{\text {comp }}=$ $\left[\Delta \delta_{\mathrm{NH}}^{2}+\left(\Delta \delta_{\mathrm{N}} / 6.5\right)^{2}\right]^{1 / 2}$ (Mulder et al., 1999). The same analysis was applied for the 
HNCO and ${ }^{15} \mathrm{~N}-{ }^{1} \mathrm{H}$ HSQC experiments when Pin1 was under investigation. For the relaxation experiments, the program PINT (Ahlner et al., 2013) was used to integrate peaks as well as to fit $R_{1}$ and $R_{10}$ rates and to calculate $R_{2}$ and the heteronuclear NOE.

\section{Derivation of constraints for structural modelling}

Both NMR CSPs and HNCO intensity ratios were used to obtain constraints in the structural modelling of the fuzzy complex. Before the experimental signals could be used as constraints in modeling the background was subtracted by thresholding. The threshold for significant $\triangle \mathrm{CSP}$ signals was set using a $\sigma$-filter with $2 \sigma$ cutoff (Schumann et al., 2007) whereas the c-Myc $\mathrm{M}_{1-88}: \operatorname{Pin} 1_{\mathrm{ww}}$ and c-Myc $\mathrm{c}_{1-8}: \operatorname{Pin} 1_{\text {PPlase }}$ HNCO ratio thresholds were set at below 0.4 in order to qualitatively reflect the observed binding pattern. $\mathrm{HNCO}$ ratios of $\mathrm{c}-\mathrm{Myc}_{1-88}$ :Pin1, that is in presence of full length Pin1, was set to 0.2. Each set of signals, above (Pin1 $\Delta \mathrm{CSP}$ ) and below (c-Myc $\mathrm{y}_{-88} \mathrm{HNCOs)}$ the thresholds was linearly rescaled between 0.0 and 1.0 from the threshold to the extreme. This resulted in 31 signals for Pin1:c-Myc $\mathrm{C}_{1-88}, 16$ signals for $\mathrm{c}-\mathrm{Myc}_{1}$ ${ }_{88}:$ Pin $1_{\mathrm{ww}}, 8$ for $\mathrm{c}-\mathrm{Myc}_{1-88}:$ Pin $1_{\mathrm{PPIase}}$ and 37 for $\mathrm{c}-\mathrm{Myc}_{1-88}:$ Pin1; for the Pin1:c-Myc $\mathrm{C}_{1-88}$ signals 11 correspond to $\operatorname{Pin} 1_{\mathrm{Ww}}\left(\operatorname{Pin} 1_{\mathrm{ww}}: \mathrm{C}-\mathrm{Myc}_{1-88}\right)$ and 20 signals to Pin $1_{\text {PPlase }}$ $\left(\operatorname{Pin} 1_{\text {PPlase }}: c-\mathrm{Myc}_{1-88}\right)$, for $\mathrm{c}-\mathrm{Myc}_{1-88}: \operatorname{Pin} 1_{\mathrm{ww}}$ and -:Pin $1_{\text {PPlase }}$ all signals corresponded to

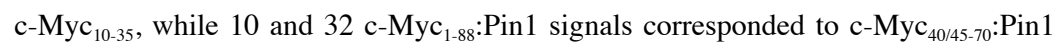
and $\mathrm{c}-\mathrm{Myc}_{10-70}:$ Pin1 respectively. The constraints for the simulations was constructed from appropriate sets of Pin1 and c-Myc $\mathrm{c}_{1-88}$ signals, by forming the cartesian product between the sets and weighing each pair in the product by the product of the separate signals; For MB0 it was Pin $1_{\mathrm{ww}}: \mathrm{c}-\mathrm{Myc}_{1-88}$ with c-Myc $\mathrm{C}_{1-88}: \mathrm{Pin}_{\mathrm{Ww}}$ and Pin1 $1_{\mathrm{PPIase}}: \mathrm{c}-\mathrm{Myc}_{1}$ ${ }_{88}$ with c-Myc $\mathrm{1}_{1-88}:$ Pin $1_{\text {PPlase }}$, for MBI Pin $1_{\text {PPlase }}: \mathrm{c}-\mathrm{Myc}_{1-88}$ with c-Myc $\mathrm{C}_{40 / 45-70}: \operatorname{Pin} 1$ and for MB0+MBI Pin1:c-Myc ${ }_{1-88}$ with c-Myc ${ }_{10-70}: \operatorname{Pin} 1$. In total, this resulted in a 336 possible interactions in the $\mathrm{c}-\mathrm{Myc}_{10-35}$ simulation, 200 for $\mathrm{c}-\mathrm{Myc}_{40 / 45-70}$ and 992 for c$\mathrm{Myc}_{10-70}$. The putative interactions were then included as constraint bonus in the scoring function using a functional form that rewarded interactions $<10 \AA$, and did not penalize non-interactions. The overall constraint weight was set to 10 and accounted for about a quarter of the total score. 
The Rosetta FlexPepDock (Raveh et al., 2011) protocol was used to dock c-Myc fragments to Pin1 guided by experimental constraints. While keeping the backbone of the Pin1 structure unperturbed, the protocol optimizes the backbone phi-/psi- angles of the c-Myc fragment and its orientation with respect to Pin1 using a Monte-Carlo with minimization approach. In the later stages of each run, the simulation optimizes the side chain orientations of residues in both Pin1 and c-Myc, minimizing steric clashes and repulsive charges, whilst backbones are kept fixed. For the full Rosetta protocol description; see (Raveh et al., 2011). This docking was followed by loop modeling, joining the best Myc MB0 and MBI fragment models into a number of joint c-Myc MB0+MBI models in an all versus all manner. The 40 best scoring joint models (rosetta score with c-Myc ${ }_{10-70}:$ Pin1 vs. Pin1:c-Myc $\mathrm{C}_{1-88}$ constraints) were subsequently used as starting structures and modeled again as a single fragment using Rosetta with the FlexPepDock protocol, yielding the final c-Myc $\mathrm{C}_{10-70}$ models.

Specifically, starting conformations were generated by placing an extended conformation of $\mathrm{c}-\mathrm{Myc}_{10-35}$ (comprising MB0) in a random orientation on Pin1 (pdb code: 1PIN) such that the Pin1 and c-Myc residues partaking in one of the experimental constraints used was superimposed on each other; this was repeated for all constraints, skipping only constraints where one or both of the separate signals was below 0.5 .. Additional starting structures were also generated by rotating $\mathrm{c}-\mathrm{Myc}_{10-35}$ by 180 degrees around the constraint superposition in all starting structures to avoid directionality biases, yielding a total of 60 starting structures. Using the same approach, two fragments was used to model c-Myc MBI; c-Myc $40-70$ and c-Myc $45-70$. Here, 40 starting structures was generated for each MBI fragment by filtering out only the constraints where the Pin1 signal was below 0.5. For each fragment, the resulting docking poses were clustered using the Rosetta clustering algorithm with a $3.0 \AA$ cluster radius. For $\mathrm{Myc}_{10-35}, 198,000$ decoys was calculated and clustered (Supplementary Figure 7), the c-Myc40-70 and c-Myc45-70 modeling yielded 200,000 structures for each fragment and was subsequently clustered separately with the same procedure as for the c-Myc MB0 models. The best models from the 20 largest clusters of the c- $\mathrm{Myc}_{10-35}$ were combined with the best models of the 20 largest clusters from both of the c-Myc $\mathrm{M}_{40-70}$ and $\mathrm{c}-\mathrm{Myc}_{45-70}$ fragments in an all versus all manner, resulting in 20x40=800 disjoint models. The combined models was then joined together in 
Rosetta using the loop modeling protocol (Qian et al., 2007; Wang et al., 2007), keeping the backbone fixed of Pin1 and only allowing the backbone of c-Myc residues 33-45 to be perturbed. The resulting models was scored using Rosetta together with the $\mathrm{c}-\mathrm{Myc}_{10-70}$ :Pin1 constraints generated as described above. By selecting the 40 best scoring of the joint models from above as starting structures and using the 992 constraints within the FlexPepDock Rosetta protocol, we generated 195,000 models of $\mathrm{c}-\mathrm{Myc}_{10-70}$ bound to Pin1. These models was then clustered in the same manner as described before, using the 50,000 best scoring models (due to computer memory constraints), yielding 61 clusters, with 40 being of significant size (shown in Supplementary Figure 8). The 40 largest clusters was then sorted according to their best scoring models and the top 17 clusters was chosen as a representative ensemble for visualization of our c-Myc $\mathrm{M}_{10-70}$ :Pin1 model.

\section{Cell culture, transfection}

HEK293 and REF52 cells were cultured as previously described (Farrell et al., 2013; Yeh et al., 2004). Specifically, cells were maintained in Dulbecco's modified Eagle's medium (DMEM) supplemented with 10\% standard fetal bovine serum (FBS), 2.5 mM L- glutamine, and 1 x penicillin-streptomycin. HEK293s were passaged every 2 days, REF52 were passaged every 3-4 days. Plating of cells was done to achieve 60$80 \%$ confluency $24 \mathrm{~h}$ post-split for transfection. All transfections were performed using Lipofectamine 2000 (Life technology, U.S.). Total transfected DNA was held constant (unless otherwise indicated) by the addition of empty control plasmid and included $50 \mathrm{ng}$ of CMV-b-gal to normalize for transfection efficiencies between experimental conditions.

\section{Western blotting and Antibodies}

Cell lysates were run on SDS-PAGE gels and transferred to Immobilon-FL membranes (Millipore). The membranes were blocked with Odyssey blocking buffer (LI-COR Biosciences, Lincoln, NE). Primary antibodies were diluted in 1:1 Odyssey blocking buffer-PBS with $0.05 \%$ Tween 20 . Primary antibodies were detected with secondary antibodies labeled with the near-infrared fluorescent dyes IRDye800 (Rockland, Philadelphia, PA) and Alexa Fluor 680 (Molecular Probes, Eugene, OR). Secondary antibodies were diluted 1:10,000 in 1:1 Odyssey blocking buffer-PBS with 
$0.05 \%$ Tween 20. Blots were scanned with an Odyssey infrared imager (LI-COR Biosciences) to visualize proteins. Antibodies used: Anti-V5 mouse monoclonal antibody (Invitrogen); Anti-FLAG M2 mouse monoclonal antibody (Sigma); Antibeta Actin antibody (ab8227); Anti-c-Myc (phosphor S62) antibody (ab78318).

Myc immunoprecipitation assays

HEK293 cells were transfected with V5 tagged Myc and other indicated plasmids for 2 days. A total of $\sim 5 \times 106$ HEK293 cells were washed with phosphate-buffered saline (PBS) once, and then resuspended in $1 \mathrm{ml}$ of co-IP buffer $(20 \mathrm{mM}$ Tris, $\mathrm{pH} 7.5,12.5 \%$ glycerol, 0.5\% NP-40, $150 \mathrm{mM} \mathrm{NaCl}, 2$ mM EDTA, 2 mM EGTA, and $1 \mathrm{mM}$ DTT) plus protease and phosphatase inhibitors. Cellular lysates were sonicated for 10 pulses (output 1; 15\% duty cycle; Branson Sonifier 450), incubated on ice for $20 \mathrm{~min}$, and cleared by centrifugation at $14,000 \mathrm{rpm}$ for $10 \mathrm{~min}$ at $4^{\circ} \mathrm{C}$. The supernatants were adjusted for transfection efficiency as measured by $\beta$-Gal activity and incubated with $2 \mathrm{ug}$ of V5 antibody for 1 hour at room temperature and then added protein ASepharose beads (Repligen, Waltham, MA) for 1 hour at $4^{\circ} \mathrm{C}$. The immunoprecipitates were washed 3 times with $1 \mathrm{ml}$ of co-IP buffer and analyzed by Western blotting for Myc phosphorylation status or other co-IPed proteins.

\section{Luciferase assays}

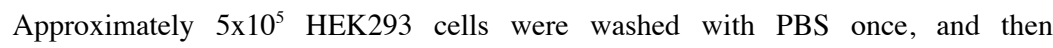
resuspended in 200ul of cell lysis buffer (Promega, Madison, WI) with protease and phosphatase inhibitors. Cellular lysates were sonicated for 10 pulses at output 1, 10\% duty cycle (Branson Sonifier 450), then incubated on ice for $20 \mathrm{~min}$. The supernatant lysates were collected after centrifugation at $14,000 \mathrm{rpm}$ for $10 \mathrm{~min}$ at $4^{\circ} \mathrm{C}$. Luciferase activity was measured using the standard Promega luciferase assay kit and a Berthold (Bundoora, Australia) luminometer. Luciferase activity was adjusted by $\beta$-Gal activity. Fold changes in luciferase activities were measured relative to empty vector or control transfections.

\section{Colony formation assay}

REF52 cells were grown about $80 \%$ confluence in p100 plates for transfection. Expression vectors containing c-Myc WT or mutant (pEntr-Dest40, $1 \mu \mathrm{g} / \mathrm{plate}$ ) and $\mathrm{H}$ - 
$\operatorname{ras}^{\mathrm{G} 12 \mathrm{~V}}$ (pBabe, $1 \mu \mathrm{g} / \mathrm{plate}$ ) were used for transfections. Where indicated, transfections were supplemented with respective empty vectors as controls. 72-hour posttransfections, cells were re-plated into p100s at low density ( $5 \mathrm{k}-25 \mathrm{k}$ cells/plate). Following re-plating, cells were maintained in DMEM containing $4 \%$ fetal bovine serum, and the colonies were visualized by staining with crystal violet blue 2 weeks later. 


\section{Supplemental References}

Ahlner, Alexandra, Mats Carlsson, Bengt Harald Jonsson, and Patrik Lundström (2013). “PINT: A software for integration of peak volumes and extraction of relaxation rates." Journal of Biomolecular NMR 56.3, pp. 191-202.

Bienkiewicz, Ewa A. and Kevin J. Lumb (1999). "'Random-coil chemical shifts of phosphorylated amino acids." Journal of Biomolecular NMR 15, pp. 203-206.

Brondani, Vincent, Quirino Schefer, François Hamy, and Thomas Klimkait (2005). "'The peptidyl-prolyl isomerase Pin1 regulates phospho-Ser77 retinoic acid receptor $\alpha$ stability." Biochemical and Biophysical Research Communications 328, pp. 6-13.

Delaglio, Frank, Stephan Grzesiek, Geerten W. Vuister, Guang Zhu, John Pfeifer, and Ad Bax (1995). "NMRPipe: A multidimensional spectral processing system based on UNIX pipes." Journal of Biomolecular NMR 6.3, pp. 277-293.

Edgar, Robert C (2004a). "MUSCLE: a multiple sequence alignment method with reduced time and space complexity." BMC bioinformatics 5, p. 113.

- (2004b). "MUSCLE: multiple sequence alignment with high accuracy and high throughput." Nucleic acids research 32.5 , pp. 1792-7.

Finn, Robert D et al. (2014). "Pfam: the protein families database." Nucleic acids research 42.1, pp. D222-30.

Lufei, C, T H Koh, T Uchida, and X Cao (2007). 'Pin1 is required for the Ser727 phosphorylation-dependent Stat3 activity." Oncogene 26, pp. 7656-7664.

Monje, Paula, Javier Hernández-Losa, Ruth J. Lyons, Maria D. Castellone, and J. Silvio Gutkind (2005). "Regulation of the transcriptional activity of c-Fos by ERK: A novel role for the prolyl isomerase Pin1." Journal of Biological Chemistry 280.42, pp. 35081-35084.

Mulder, F a, D Schipper, R Bott, and R Boelens (1999). “Altered flexibility in the substratebinding site of related native and engineered high-alkaline Bacillus subtilisins." Journal of molecular biology 292.1, pp. 111-123.

Sattler, M (1999). "Heteronuclear multidimensional NMR experiments for the structure determination of proteins in solution employing pulsed field gradients." Progress in Nuclear Magnetic Resonance Spectroscopy 34.2, pp. 93-158.

Schuster-böckler, Benjamin, Jörg Schultz, and Sven Rahmann (2004). 'HMM Logos for visualization of protein families." BMC bioinformatics 8, pp. 1-8.

Suzek, Baris E, Hongzhan Huang, Peter McGarvey, Raja Mazumder, and Cathy H Wu (2007). "UniRef: comprehensive and non-redundant UniProt reference clusters." Bioinformatics (Oxford, England) 23.10, pp. 1282-8.

Wang, Chu, Philip Bradley, and David Baker (2007). “Protein-Protein Docking with Backbone Flexibility." Journal of Molecular Biology 373.2, pp. 503-519.

Wheeler, Travis J, Jody Clements, and Robert D Finn (2014). ' Skylign: a tool for creating informative, interactive logos representing sequence alignments and profile hidden Markov models." BMC bioinformatics 15.1, p. 7.

Wulf, Gerburg M., Akihide Ryo, Gerald G. Wulf, Sam W. Lee, Tianhua Niu, Victoria Petkova, and Kun Ping Lu (2001). "Pin1 is overexpressed in breast cancer and cooperates with Ras signaling in increasing the transcriptional activity of c-Jun towards cyclin D1." EMBO Journal 20.13, pp. 3459-3472.

Yaffe, Michael B. et al. (1997). ' Sequence-Specific and Phosphorylation-Dependent Proline Isomerization: A Potential Mitotic Regulatory Mechanism." Science 278, pp. 1957-1960. 


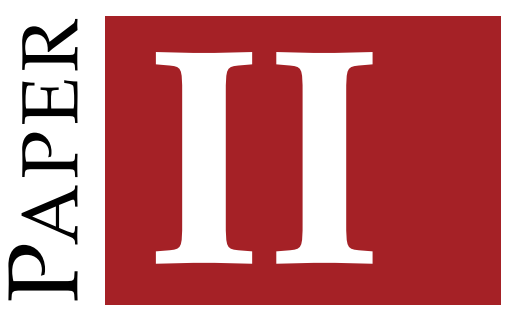

Madhanagopal Anandapadamanaban, Robert Pilstål, Cecilia Andresen, Jill Trewhella, Martin Moche, Björn Wallner, and Maria Sunnerhagen. "Mutation-Induced Population Shift in the MexR Conformational Ensemble Disengages DNA Binding: A Novel Mechanism for MarR Family Derepression". en. In: Structure 24.8 (Aug. 2016), pp. 1311-1321. ISSN: 09692126. DOI: $10.1016 / j . s t r .2016 .06 .008$ URL:

http://linkinghub.elsevier.com/retrieve/pii/S0969212616301332(visited on $04 / 11 / 2017)$ 



\section{Structure}

Mutation-Induced Population Shift in the MexR Conformational Ensemble Disengages DNA Binding: A Novel Mechanism for MarR Family Derepression

Graphical Abstract

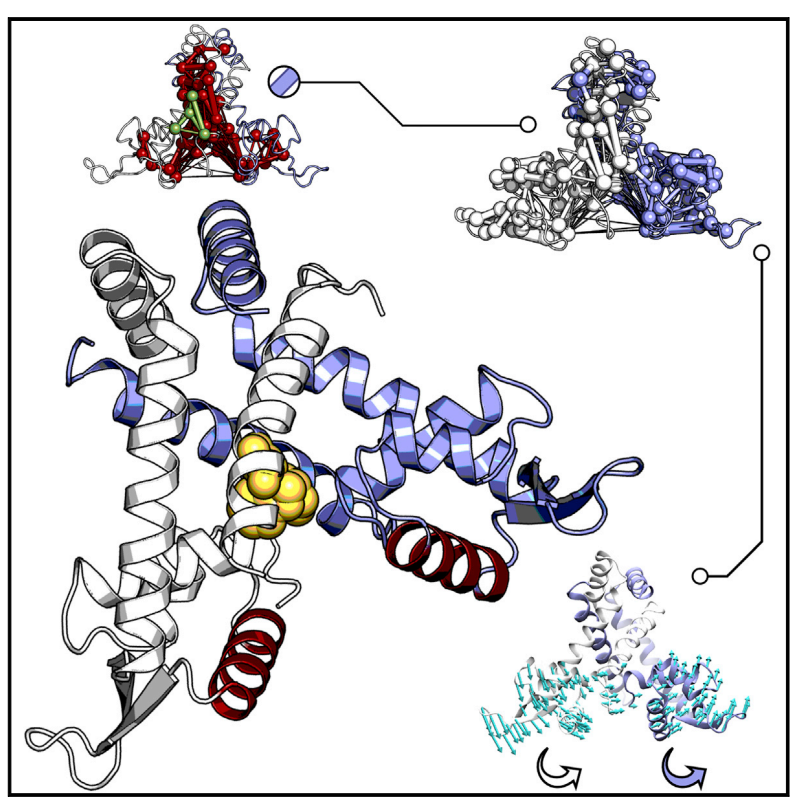

Highlights

- First high-resolution structure of a multidrug-resistanceconferring MexR mutant

- MexR-R21W shows evidence for a more compacted, less dynamic structure than MexR-wt

- The MexR-R21W conformational ensemble excludes DNAbinding conformations

- Collapsed allosteric networks disable independent motion of DNA-binding domains
Authors

Madhanagopal Anandapadamanaban, Robert Pilstål, Cecilia Andresen, Jill Trewhella, Martin Moche, Björn Wallner, Maria Sunnerhagen

\section{Correspondence}

maria.sunnerhagen@liu.se

In Brief

By analyzing a multidrug-resistanceconferring mutation in MexR, Anandapadamanaban, Pilstål et al. highlight a new mechanism for allosteric regulation without structural change. While structures of mutant and wild-type are similar, DNA binding is disrupted by restricting the free-state conformational ensemble and by collapsed allosteric networks.

\section{Accession Numbers}

4ZZL 


\title{
Mutation-Induced Population Shift in the MexR Conformational Ensemble Disengages DNA Binding: A Novel Mechanism for MarR Family Derepression
}

\author{
Madhanagopal Anandapadamanaban, ${ }^{1,5}$ Robert Pilstål, ${ }^{2,5}$ Cecilia Andresen, ${ }^{1}$ Jill Trewhella, ${ }^{1,3}$ Martin Moche, \\ Björn Wallner, ${ }^{2,6}$ and Maria Sunnerhagen ${ }^{1,6, *}$ \\ 1Division of Chemistry, Department of Physics, Chemistry and Biology \\ 2Division of Bioinformatics, Department of Physics, Chemistry and Biology \\ Linköping University, 58183 Linköping, Sweden \\ ${ }^{3}$ School of Molecular Bioscience, The University of Sydney, Sydney, NSW 2006, Australia \\ ${ }^{4}$ Department of Medical Biochemistry and Biophysics, Protein Science Facility, Karolinska Institutet, 17177 Stockholm, Sweden \\ ${ }^{5}$ Co-first author \\ ${ }^{6}$ Co-senior author \\ *Correspondence: maria.sunnerhagen@liu se \\ http://dx.doi.org/10.1016/j.str.2016.06.008
}

\section{SUMMARY}

MexR is a repressor of the MexAB-OprM multidrug efflux pump operon of Pseudomonas aeruginosa, where DNA-binding impairing mutations lead to multidrug resistance (MDR). Surprisingly, the crysta structure of an MDR-conferring MexR mutant R21W $(2.19 \AA)$ presented here is closely similar to wildtype MexR. However, our extended analysis, by molecular dynamics and small-angle X-ray scattering, reveals that the mutation stabilizes a ground state that is deficient of DNA binding and is shared by both mutant and wild-type MexR, whereas the DNA-binding state is only transiently reached by the more flexible wild-type MexR. This population shift in the conformational ensemble is effected by mutation-induced allosteric coupling of contact networks that are independent in the wild-type protein We propose that the MexR-R21W mutant mimics derepression by small-molecule binding to MarR proteins, and that the described allosteric mode based on population shifts may also apply to other MarR family members.

\section{INTRODUCTION}

The MarR (multiple antibiotic resistance regulator) family of prokaryotic transcriptional regulators includes proteins critical for control of virulence factor production, bacterial response to antibiotic and oxidative stresses, and catabolism of environmental aromatic compounds (Wilkinson and Grove, 2006). Proteins belonging to the MarR family are particularly critical for the survival of pathogenic bacteria in hostile environments. As an example, Pseudomonas aeruginosa is a Gram-negative bacterium and an opportunistic human pathogen, largely thanks to its efficient efflux systems that actively expel substances that are toxic to the bacterium across the cell wall (reviewed in Perera and Grove, 2010). Among these, the expression of the mexABoprM efflux pump is negatively regulated by the MarR family protein MexR, but is activated by MexR binding of a particularly wide range of in vivo effector molecules, which relieve MexR of DNA binding (Li et al., 1998; Poole, 2001; Poole et al., 1996), as well as by the binding of the regulatory ArmR protein (Wilke et al., 2008) and by oxidation (Chen et al., 2010a). Constitutive expression of efflux pump proteins by mutations in MexR leads to multiple antibiotic/drug resistance (MAR/MDR) (Cao et al., 2004; Srikumar et al., 2000; Ziha-Zarifi et al., 1999). Structurally, MexR is a member of the homodimeric MarR family of transcriptional regulators, which includes two winged helix-turn-helix (wHTH) DNA-binding domains that are joined by a helical dimerization domain (reviewed in Perera and Grove, 2010; Wilkinson and Grove, 2006). MexR structures are available for the unliganded apo state (Lim et al., 2002), and for two structural states that are inhibited with respect to DNA binding: the ArmR complex (Wilke et al., 2008) and the oxidized form (Chen et al., 2010a). Although there is still no structure for DNA-bound MexR, recent structures of the close homologs OhrR (Hong et al., 2005) and MepR (Birukou et al., 2014) are available for comparison.

For turning on the efflux pump machinery, a key molecular feature of most MarR family members is their ability to bind, and thus respond, to highly diverse external signals, from molecules to ions (Wilkinson and Grove, 2006). Structures of Bacillus subtilis OhrR in apo, DNA-bound, and inducer-bound states have been jointly interpreted to confer regulatory activity in a domino-like, allosteric cascade of conformational changes (Hong et al., 2005); similar effector-induced rigid-body displacements have since been suggested for a range of MarR family proteins (Alekshun et al., 2001; Birukou et al., 2013; Chang et al., 2010; Dolan et al., 2011; Kumarevel et al., 2009; Radhakrishnan et al., 2014; Saridakis et al., 2008). Regulation of DNA binding also responds to stress-induced cellular changes, which induce cysteine-crosslinked inactive conformations for both MarR and MexR (Chen et al., 2008). Preconfigured DNA binding has been observed for the uric acid-binding HucR in its apo state 
(Perera et al., 2009), and for AdcR in its $\mathrm{Zn}^{2+}$-bound state (Guerra et al., 2011). However, the stringent, domino-like responses proposed for the MarR family (Hong et al., 2005) contrast with the structural versatility required to accommodate binding of the various inducer molecules that turn on efflux pump expression. Furthermore, the MarR family of proteins is exposed to significant selective pressure for mutations that incapacitate DNA binding and thereby result in MDR (Grove, 2013). Interestingly, the MarR family displays MDR mutations that are scattered over the entire protein scaffold with maintained folding, rather than being sensitive to mutations at selected, specific sites within the domino pathways (Alekshun et al., 2000; Andrésen et al., 2010; Birukou et al., 2013), and similarly scattered mutation patterns have been observed for MexR (Ade woye et al., 2002; Jalal et al., 1999). A highly diverse biophysical response pattern (Andrésen et al., 2010; Birukou et al., 2013) suggests that the MarR family of proteins is able to structurally respond in highly varied ways that allosterically effectuate DNA derepression.

To help our understanding of MarR family derepression mechanisms, we need to consider that the dynamic scope within the MarR family may be wider than previously assumed, with structural versatility playing an important functional role. While the crystal structure of MexR revealed four similar but structurally distinct homodimers (Lim et al., 2002), MexR incapacitated for DNA binding by oxidation (Chen et al., 2010a) or by binding to the inhibitory protein ArmR (Wilke et al., 2008) shows a single conformation in respective crystal structures. Similarly, multiple conformational states were recently revealed in the crystal structure of Staphylococcus aureus MepR bound to DNA, which was reported with three different homodimer complexes (Birukou et al., 2014). Furthermore, biophysical evidence suggests that regulatory ligand binding may affect interdomain dependence: MftR exhibits a two-step melting transition, suggesting independent unfolding of the dimerization and DNA-binding regions, while urate binding or mutations in the predicted ligand-binding sites result in one-step unfolding transitions, suggesting more tightly linked dimerization and DNA-binding domains (Gupta and Grove, 2014). In MexR, interdomain communication could be inferred from $T_{m}$ transitions, where single point mutations stabilizing the dimer region also elevate the stability of the DNAbinding domains (Andrésen et al., 2010).

To investigate how MDR mutants in the MarR family affect DNA binding, we studied the MexR-R21W protein by crystallography, and both the mutant and wild-type MexR (MexR-wt) proteins by molecular dynamics (MD) simulations in silico and by small-angle X-ray scattering (SAXS) in solution. From a well-established set of MexR mutations conferring MDR (Jalal et al., 1999; Adewoye et al., 2002) we chose to further study MexRR21W, mutated in the dimerization domain (Figure 1A), for which we have observed retained structure content and significantly increased stability, but concomitant loss of DNA binding (Andrésen et al., 2010). Our results suggest a regulatory path for MexR whereby the R21W mutation stabilizes a non-DNA-binding ground state by dynamic and allosteric blocking of access to the DNA-binding state. Since small-molecule binding essentially in any groove or cavity of the ground state would effect similar stabilization, our finding would explain the noted versatility in derepressing ligand binding that is displayed in the MarR family.
We propose that allosterically effected population shifts such as we describe here for MexR may indeed be commonly occurring on mutation and/or ligand binding within other MarR family members, and need to be taken into consideration in developing future drug-design strategies to combat multiple drug resistance.

\section{RESULTS}

MexR-R21W: A Combined Experimental and

Computational Approach

Experimental structural biology and MD simulation were jointly used to investigate the structure and dynamics of MexR-R21W and MexR-wt, with the aim of learning how this mutation can negatively affect DNA binding. Crystallography successfully yielded a high-resolution structure of MexR-R21W refined to a final resolution of $2.19 \AA$, and with a single complete homodimer present in the crystallographic unit cell (Figure 1A). Crystals were obtained through an iterative seeding procedure, where only the His-tagged MexR-R21W comprising residues 5-139 yielded crystals that diffracted to high resolution (see Experimental Procedures). Data collection and refinement statistics are provided in Table 1. SAXS was employed to experimentally assess the structural space occupied by MexR-R21W and MexR-wt in solution and to identify sample conditions for pure homodimers with no aggregation (Table S1). Nuclear magnetic resonance (NMR) was attempted under these conditions, but a complete evaluation was hindered by the low concentrations $(60 \mu \mathrm{M})$ required to avoid self-association. However, extensive line broadening in heteronuclear single-quantum coherence spectra of MexR$\mathrm{R} 21 \mathrm{~W}$, and even more so for MexR-wt, suggested the presence of $\mu \mathrm{s}-\mathrm{ms}$ dynamics (Figure S1). To enable further investigation of the intrinsic dynamics of the homodimer, we used the MexR$\mathrm{R}_{21} \mathrm{~W}_{5-139}$ and MexR-wt crystal structures using the same extent of residues (5-139) as starting models for MD simulations. A total of ten independent 100-ns MD trajectories were calculated starting from each of four different MexR structures, namely MexR-wt, -CD, -EF, and -GH dimers (Lim et al., 2002), and the MexR-R21W structure presented here $(10 \times 4 \times$ 100-ns simulation time). The MexR-wt AB dimer was omitted from this study, since this crystal model binds the C-terminal tail of another MexR molecule between its DNA-binding domains (Lim et al., 2002), likely dominating its dynamics.

The MexR-R21W Crystal Structure Is Highly Asymmetric and Similar to MexR-wt

Overall, the MexR-R21W crystal structure retains the classical MarR protein fold (Figure $1 \mathrm{~A}$ ) and is highly similar to the structures of MexR-wt (Lim et al., 2002) (Figure S2). Furthermore, similar to MexR-wt, MexR-R21W shows significant asymmetry with respect to the monomer pairs constituting each dimer. A distinguishing feature of the asymmetry is found in the sidechain orientation of Phe17/17' and, to a lesser degree, in that of Leu13/13' (Figures 1B and S3). Both Leu13 and Phe17 are located in $\mathrm{H} 1 / \mathrm{H} 1^{\prime}$ at the dimer interface. Notably, Phe17/17' residues would clash in a completely symmetric structure but in the asymmetric MexR structures, Phe17 either points away from $\mathrm{H} 6 / \mathrm{HG}^{\prime}$ in what we name as a (-) chain, or toward $\mathrm{H} 6 / \mathrm{H}^{\prime}$ in a (+) chain (Figures 1B and S3). We observed previously that 
A

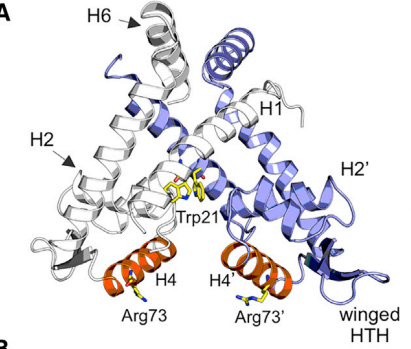

B

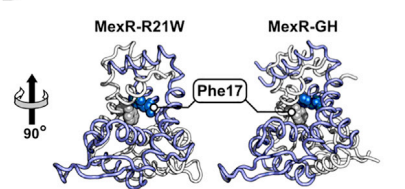

ㄷ) (다)

C
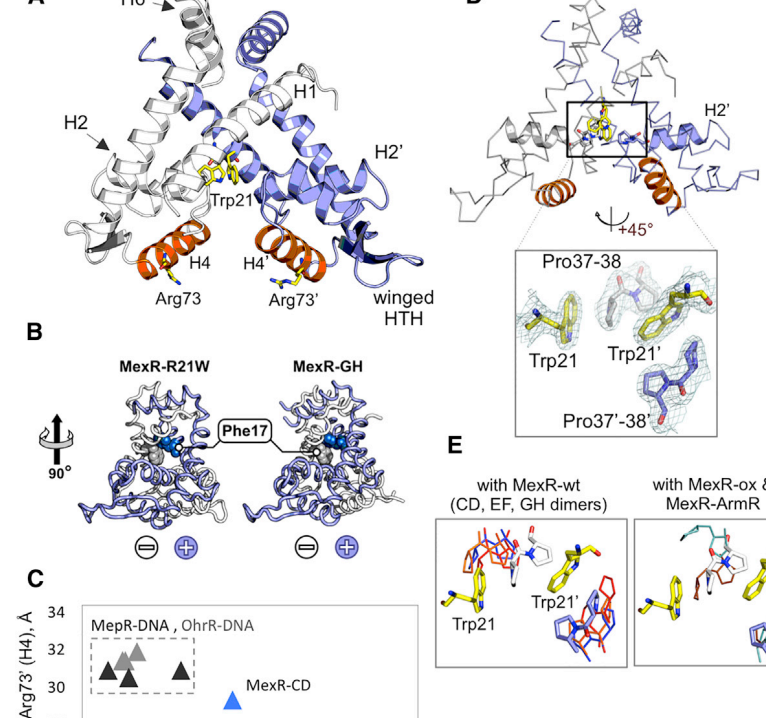

Trp21

E

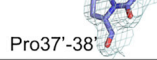

with MexR-wt

with MexR-ox
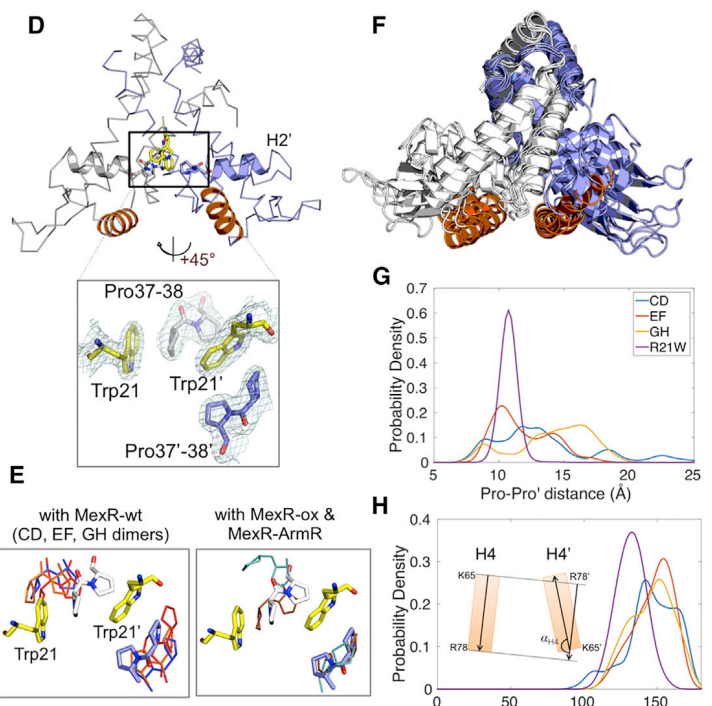

G

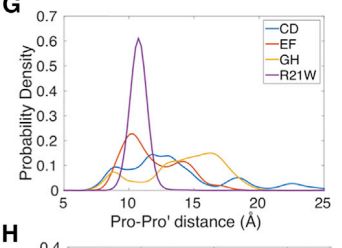

H

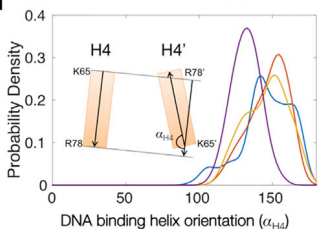

Figure 1. Crystal Structure of MexR R21W and Pro37-38 Cavity

(A) The MexR-R21W dimer structure (gray and blue) featuring Trp21 and Arg73 (labeled), and the DNA recognition helices ( $\mathrm{H} 4$ and $\mathrm{H}^{\prime}$; orange).

(B) MexR-R21W and MexR-wt (GH dimer) showing the annotation of $(-)$ and (+) chains, with residue Phe17 highlighted.

(C) Graphical plot of asymmetry (André et al., 2008) versus midpoint interdistance of H4-H4' for MexR (PDB: 1LNW), MexR-R21W (this study), MexR-ox (PDB: 3MEX), MexR-ArmR (PDB: 3ECH), and DNA-bound MepR (black; PDB: 4LLN) and OhrR (gray; PDB: 1Z9C). Distances were measured between $\mathrm{C}_{\alpha} \mathrm{S}$ of $\mathrm{Arg} \mathrm{A3} / 73^{\prime}$ in MexR and between corresponding atoms in MepR and OhrR dimers.

(D) MexR-R21W structure highlighting H2/2', H4/4', and residues Pro37, Pro38, and Trp21 in both chains. Inset (tilted $+45^{\circ}$ ) shows the $2 \mathrm{~F}_{\mathrm{O}}-\mathrm{F}_{\mathrm{C}}$ map (gray mesh) of Trp21 and Pro37-38 residues in both chains contoured at 1.2 $\sigma$. Colors as in (A).

(E) Magnified view of Pro37-38 region of MexR-wt (CD, EF, and GH; shown in lines with colors as in C superimposed onto MexR-R21W [shown as sticks, color and orientation as in Dl]. The adjacent inset similarly shows the comparison with MexR-oxidized and MexR-ArmR. Colors as in (C).

orientation as in D]). The adjacent inset similarly shows the comparison with MexR-oxidized and MexR-ArmR. Colors as in (C).
(F) Structural representatives for the five largest clusters from the simulation trajectories, superimposed on the dimerization region.

(F) Structural representatives for the five largest clusters from the simulation trajectories, superimposed on the dimerization region. (G and H) The Pro37-38 distance distribution (G) and the

See also Figures $\mathrm{S} 1-\mathrm{S} 4$.

mutation of Leu13 results in an unstable protein (Andrésen et al., 2010), reflecting a possible structural importance also of this residue. To take the asymmetry into account in structural comparisons between MexR-R21W and MexR-wt, we sorted the chain order by performing single-linkage clustering based on the pairwise $\mathrm{C}_{\alpha}$ root-mean-square deviation (RMSD). This revealed that the chain order of the EF dimer had to be flipped to structurally fit with the $C D$ and GH dimers (Figure S3). For MexR-R21W, its AB chain order is most similar to wild-type structure cluster $\mathrm{CD}, \mathrm{GH}$, and $\mathrm{FE}$, with a backbone average RMSD of $2.2 \AA$ ( $\mathrm{AB}$ ) compared with $2.6 \AA(B A)$ for the entire proteins, and 1.0-1.3 $\AA$ ( $A B$ ) for the dimerization domain alone (Figure S3).
While MexR has not yet been crystallized in its DNA-bound state, the DNA-bound forms of closely homologous MarR family members OhrR and MepR both show significantly lower asymmetry, as do the DNA-binding-inhibited ArmR-bound MexR and the oxidized MexR structures (Figures $1 \mathrm{C}$ and S2). Previous studies have used the inter-DNA domain spacing measured by the $\mathrm{C}_{\alpha}-\mathrm{C}_{\alpha}$ distance between Arg73 and Arg73' in the DNA-binding helices $\mathrm{H} 4 / \mathrm{H} 4^{\prime}$ to assess the ability to bind DNA (Lim et al., 2002; Wilke et al., 2008). We find that these interhelix distances in MexR-R21W are similar to those in free MexR-wt, but that both are smaller than those observed in the DNA-bound structures of the homologous OhrR and MepR (Figure 1C).

Structure 24, 1311-1321, August 2, 20161313 


\begin{tabular}{|c|c|}
\hline & MexR R21W ${ }^{a}$ \\
\hline \multicolumn{2}{|l|}{ Data Collection } \\
\hline Space group & $P 3_{2} 21$ \\
\hline Unit cell $(\AA)$ & $84.88,84.88,114.187$ \\
\hline Unit cell $\left(^{\circ}\right)$ & $90,90,120$ \\
\hline Resolution (Å) & $57.09-2.19(2.31-2.19)$ \\
\hline$R_{\text {meas }}$ & $0.093(0.918)$ \\
\hline$|/ \sigma|$ & $24.9(4.7)$ \\
\hline Completeness (\%) & $99.8(98.9)$ \\
\hline Redundancy & $21.2(21.2)$ \\
\hline Beamline & i24, Diamond \\
\hline \multicolumn{2}{|l|}{ Refinement } \\
\hline Resolution ( $(\AA)$ & $45.09-2.19(2.28-2.19)$ \\
\hline No. of reflections & $24,921(2,640)$ \\
\hline$R_{\text {work }} / R_{\text {free }}$ & $19.13 / 20.62(21.7 / 26.9)$ \\
\hline \multicolumn{2}{|l|}{ No. of atoms } \\
\hline Protein & 2,297 \\
\hline Ligand/ion & 12 \\
\hline Water & 126 \\
\hline$B$ factors & 45.09 (Wilson plot) \\
\hline Protein & 52.4 \\
\hline Water & 56.0 \\
\hline Glycerol & 74.7 \\
\hline \multicolumn{2}{|l|}{ RMSD } \\
\hline Bond lengths $(\AA)$ & 0.010 \\
\hline Bond angles $\left({ }^{\circ}\right)$ & 0.98 \\
\hline PDB ID & $4 Z Z L$ \\
\hline
\end{tabular}

Values in parentheses are for highest-resolution shell.

${ }^{a}$ Single crystal was used for data collection and refinement.

The MexR-R21W Mutation Confers Conformational Restriction in the Dimer Core

The R21W mutation site is located at the edge of the MexR dimer interface, in close proximity to the DNA-binding interface (Figure 1D). In the crystal structure of MexR-R21W the most prominent interactions to Trp21 are residues Pro37 and Pro38, which both stack with Trp21 in each of the MexR-R21W chains (Figure 1D). The corresponding Arg21 in MexR-wt points into a sizable interdomain cavity and shows few side-chain interactions ( Lim et al., 2002). Stacking of Pro-Trp has recently been found to stabilize protein interactions both in theoretical models (Biedermannova et al., 2008) and in experimental studies (Slutzki et al., 2013 and references therein) and would be in agreement with the higher stability observed for MexR-R21W compared with MexR-wt (Andrésen et al., 2010). In MexR-wt dimers, Pro37 and Pro38 are slightly more widely separated relative to their symmetry-related Pro37' and Pro38' (Figure 1E). We note that the closer packing observed in MexR-R21W is also observed for the MexR-oxidized structure, which also does not bind DNA (PDB: 3MEX), and, partly, in ArmR-bound MexR (Figure 1E) (Chen et al., 2010a; Wilke et al., 2008).

In contrast, the MD simulations of both MexR-R21W and MexR-wt reveal extensive conformational spread in silico (Fig- ure $1 F)$. This observation agrees with the broadened features of the NMR spectra in solution, indicating extensive dynamics (Figure S1). The conformational spread particularly affects the edge of the dimer interface connecting to the wHTH domains, where the R21W mutation is positioned, while the dimer region and $\mathrm{wHTH}$ domains by themselves remained intact throughout all of the MD simulations, for both MexR-wt and MexR-R21W (Figure 1F). Specifically, simulations starting from the MexR-wt structure show an extensive distance spread of 2-24 $\AA$ within the Pro37, 38, 37' $38^{\prime}$ cluster, whereas the similar distance range in MexR-R21W was confined to 9-12 $\AA$ (Figure 1G). Thus it appears that MexR-R21W can only access part of the Pro-Pro distance space accessible to MexR-wt.

To assay whether the conformational restriction in the MexR$\mathrm{R} 21 \mathrm{~W}$ core also restricts the orientation of the DNA-binding helices, we first compared the $\mathrm{H} 4 / \mathrm{H} 4^{\prime}$ distance (Arg73-Arg73') of MexR-wt and MexR-R21W in the simulated ensembles, but found no significant differences in this measure (Figure S4). Notably, however, this distance measure alone does not capture the specific relative orientation of the helices required for end-on binding into the major groove as in the MarR family (Figure S2) (Hong et al., 2005). When we instead observed the distribution of the interhelix orientation of the DNA-binding helices $(\mathrm{H} 4$, $H 4^{\prime}$ ) in the simulated ensemble, we found a significantly narrower distribution for MexR-R21W compared with MexR-wt. In particular, the MexR-R21W distribution partially excluded the range of interhelix angles that would be expected for DNA-binding conformations, e.g., $145^{\circ}-152^{\circ}$ for OhrR and MepR (Figures $1 \mathrm{H}$ and S4). In contrast, the MexR-wt distributions comprised a wide span of interhelix orientations, including those observed for MexR-R21W but also angles compatible with DNA binding (Figures $1 \mathrm{H}$ and S4). Thus, although the span of interhelix distances for the DNA-binding helices $\left(\mathrm{H} 4, \mathrm{H} 4^{\prime}\right)$ is similar for MexR-wt and MexR-R21W, it appears that MexR-R21W can only access a limited part of the interhelix angles accessible to MexR-wt, and that this angle space is distinct from that adopted by the homologous OhrR and MepR when bound to DNA. However, since both interhelical distances and angles affect interhelix positioning, neither of these measures were alone sufficient to describe DNA-binding compatibility (Figure S4), and we therefore proceeded to analyze the full conformational and dynamic space.

MexR-R21W Simulations Only Rarely Visit the Conformational Space Compatible with DNA Binding To analyze the entire conformational space spanned by the dynamic MexR-wt and MexR-R21W protein structures, we performed principal component analysis using the Essential Dynamics Analysis in ProDy (Bakan et al., 2011), over the complete trajectories for both MexR-wt and MexR-R21W simulations. We first analyzed the trajectories of MexR-wt and MexR-R21W separately, capturing $65 \%$ and $68 \%$ of the structural variation over the two largest principal components. Possibly due to the asymmetry of the MexR structure, the principal dynamics of MexR-wt shows a slightly different magnitude in the $(+)$ and $(-)$ chains, with (+) chains showing a larger range of mobility (Figure 2A). The principal dynamics of MexR-R21W shows similar features, but did not exhibit as large a dynamical difference between the two chains (Figure 2A). The similar character of the 
A
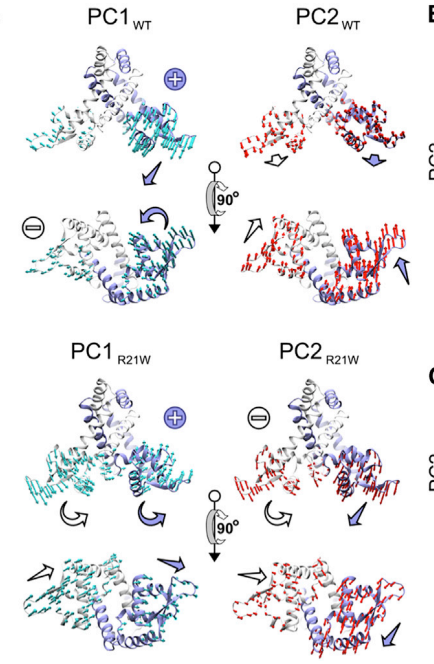

C
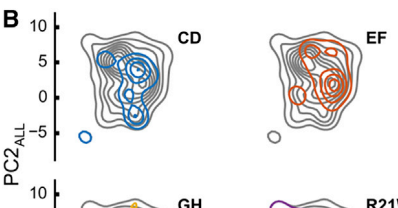

R21W
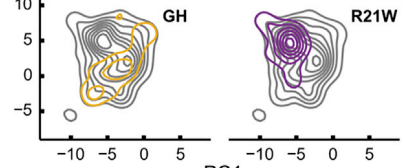$$
\mathrm{C} 1_{\mathrm{AL}}
$$

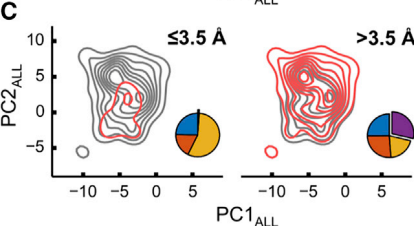

CD

$\bigcirc \mathrm{GH}$

R21W

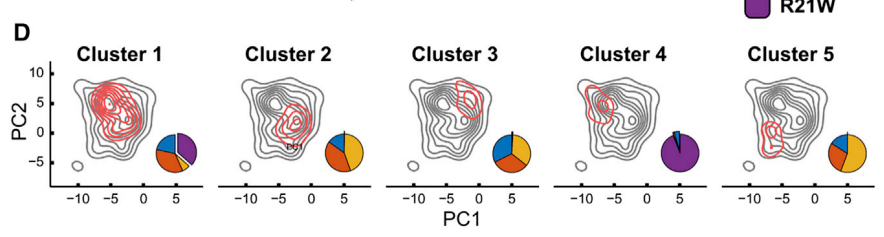

Figure 2. Essential Dynamics and Conformational Spread

(A) Essential dynamics analysis done separately on wild-type (top, WT) and mutant (bottom, R21W) simulations. The first principal component (PC1) is simulations. The firt principal component (PC1) is shown in the coll the right column for each analysis (WT and R21W), both displayed with two representations. Arrows on structure show the principal direction for each residue of significant perturbation, and stylized arrows show the perceived concerted movements of the corresponding chains as described by the respective principal component.

(B) Contour densities of projections of all simulations combined, accounting for $65 \%$ of the structural variation. The summed distribution of all trajectories are plotted as gray with subsets $C D$, $\mathrm{EF}, \mathrm{GH}$, and R21W as colored contours on top, normalized with respect to the total (gray) density. (C) Distribution of conformations with less (red, top) and more (red, bottom) than $3.5 \AA$ RMSD to DNA-binding reference structure. Pie charts show how often trajectories from each starting confow how often trajectories from each starting conformation visit each RMSD bin projection; ordered as
contour plot. contour plot.

(D) Cluster population of $\mathrm{PC} 1 / \mathrm{PC} 2$ projections for the five largest clusters (red) with projections of all conformations as background (gray). Pie charts indicate how often simulations from all starting structures visited each of the five largest clusters. See also Figures $\mathrm{S} 3-\mathrm{S} 5$. principal dynamics allowed for a joint principal component analysis of the wild-type and mutant proteins by pooling the trajectories of MexR-wt and MexR-R21W.

The structural variation, or conformational spread, in the pooled analysis can be represented by projecting all conformations on the first two principal components, PC1 and PC2, which captures $61 \%$ of the total structural variation (Figure 2B). Clearly, simulations starting from any of the MexR-wt dimers (CD, EF, or $\mathrm{GH}$ ) have a significantly larger conformational spread than corresponding simulations with MexR-R21W as a starting structure. Furthermore, while most of the MexR-R21W conformational space appears to be accessible also to MexR-wt, only part of the conformational space accessible to MexR-wt is populated by MexR-R21W. This is also evident in a more detailed analysis performed by clustering conformations over all trajectories according to their pairwise RMSD values, which captured $74 \%$ and $90 \%$ of all conformations in the five and ten largest clusters, respectively (Figure S5). Simulations starting from MexR-wt (CD, $\mathrm{EF}$, or $\mathrm{GH}$ ) and MexR-R21W visited the largest conformational cluster equally often (Figure 2D). The only cluster that is dominated by MexR-R21W, cluster 4, is also visited in the MexR-wt simulation starting from the CD dimer. In contrast, other clusters are never (clusters 2 and 5), or very rarely (cluster 3 ) visited by MexR-R21W. Together, this analysis of the entire conformational space accessed by the two proteins supports the hypothesis of a restricted conformational access for MexR-R21W compared with MexR-wt.
To evaluate whether the different population propensity of the accessible clusters could be related to their DNA-binding propensity, we sorted the conformations from all trajectories with respect to the backbone RMSD similarity of the DNA-binding helices $\left(\mathrm{H} 4, \mathrm{H} 4^{\prime}\right)$ with those of OhrR in complex with DNA and cataloged them in two bins, as similar or dissimilar to OhrR (Figure 2C). Conformations with high similarity to the DNA-bound complex cluster in a specific area of the projected conformational space, which is distinctly different to the space occupied by MexR-R21W, whereas conformations with helix orientations dissimilar to the DNA-bound state are widely dispersed over the principal component space. Notably, we found that while simulations starting from MexR-wt structures CD, FE, and GH all visited conformations with a DNA-binding helix orientation similar to that of OhrR, the MexR-R21W trajectories almost never did (Figure 2C). Taken together, the principal component analysis of dynamic motions throughout the simulated trajectories support a restricted access to the overall conformational space that would enable DNA binding for MexR-R21W compared with MexR-wt.

The MexR-R21W Mutation Couples Distinct Allosteric Networks that Are Independent in MexR-wt

We analyzed the protein structure networks in the trajectories of MexR-wt and MexR-R21W by representing the residue-residue contact network by a probabilistic graph model, and subjecting it to community partitioning using the Protein Structure Network 


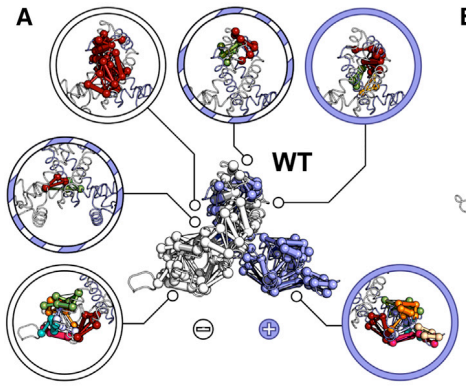

C

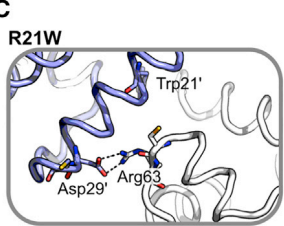

Oxidized
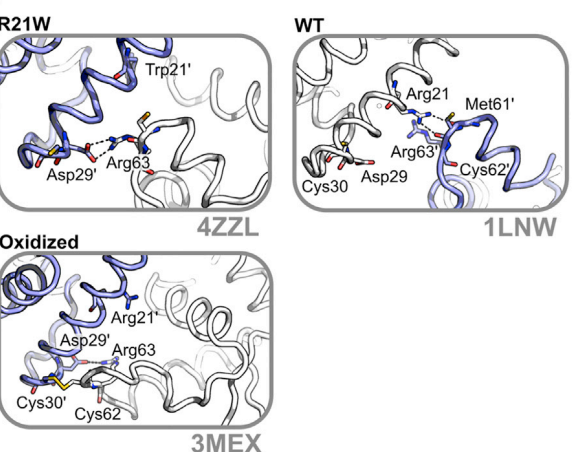

D

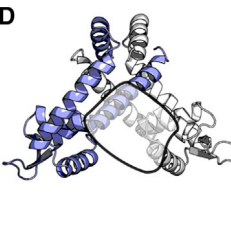

B

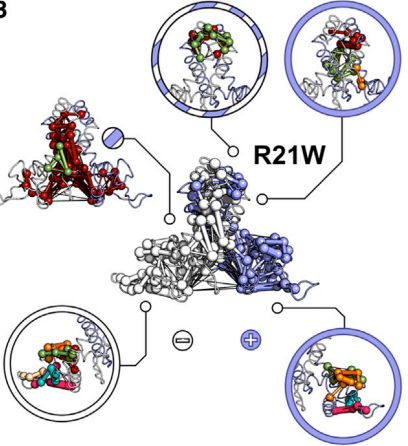

Figure 3. Protein Structure Network Clus-

Side-Chain Interactions and Crystal Observations of Wild-Type and Mutant MexR

(A and B) Wild-type (A) and mutant (B) clusters of residues that are in contact throughout most of the simulation are shown as connected spheres, with thickness of connections signifying relative interaction propensity. To enable inspection of individual clusters, these are colored separately and displayed in circles as indicated on the central views; the blue, white, or striped perimeter color indicates whether the clusters predominantly belong to the (-) (t), or both chain confominantly The most significant differences The most signicant diferences between wildtype and mutant MexR is observed in the size and shape of the clusters connecting the dimerization and DNA-binding domains.

(C) Crystal structure observations; wild-type (PDB: 1LNW) open (EF) conformations with arginine stacking highlighted, mutant (PDB: 4ZZL) in closed conformations highlighting Asp29' and Arg63 interaction, oxid Arg63 interaction, oxidized (PDB: 3MEX) showing cysteine binding in closed conformation

(D) Area of magnification in (C) on the MexR structure. Chain coloring (blue and gray) respectively corresponds to $(+)$ and $(-)$ conformations. See also Table S2 and Figure S6.

structure, Arg63 instead forms electrostatic interactions with Asp29 of the opposing chain, thus providing a double-hydrogen bond linkage that may more strongly anchor the DNA-binding

module in Wordom (Seeber et al., 2011). The protein structure network reveals to what extent residues are able to communicate via allostery; residues that are strongly allosterically connected end up in the same community, while others less prone to communicate end up in separate communities.

There are clear differences in the community boundaries between MexR-wt and MexR-R21W. In MexR-wt, we find that the communities in the wHTH domains are disconnected and independent from the ones in the dimerization domain of the protein (Figure $3 \mathrm{~A}$ and Table S2). In contrast, for R21W, parts of the core networks in both of the wHTH domains collapse into a large supercommunity together with the $(-)$ chain of the dimerization domain (Figure 3B and Table S2). The collapse of many smaller communities into one large community for the mutant essentially means that those regions of the molecule are more rigid and tightly packed, over the course of the simulation, than they are for wild-type. This result is in agreement with the observation of increased thermal stability for MexR-R21W compared with MexR-wt (Andrésen et al., 2010).

Further detailed analysis of the available MexR crystal structures revealed molecular features in agreement with a community collapse in MexR-R21W. In all MexR-wt structures, Arg63 shows solvent-mediated interactions with Arg21 in $\mathrm{H} 1$, which, together with Arg21 side chain-backbone hydrogen bonds, connect the wHTH H3/H4 loop with the $\mathrm{C}$ terminus of helix 1 in the dimerization domain (Figure $3 \mathrm{C}$ ). In the MexR-R21W crystal domain toward the dimer fold (Figure $3 C$ ).
zed MexR (MexR-ox), the covalently conInterestingly, in oxidized MexR (MexR-ox), the covalently con-
nected cysteines 62 and 30 provide a stable linkage between the two domains in the same region (Figure $3 \mathrm{C}$ ), which confers loss of DNA binding despite high structural similarity between MexR-ox and MexR-wt (Chen et al., 2010a). The structural analysis together with the dynamics analysis thus supports that progressively stronger interdomain contacts in MexR-wt, MexR$\mathrm{R} 21 \mathrm{~W}$, and MexR-ox may alter the independent motions of the wHTH domains relative to the dimer region. By collapsing previously independent allosteric communities, this progressive reduction of conformational space may in turn block access to the more widely separated $\mathrm{wHTH}$ orientations that are required to bind DNA.

MexR Samples a Significantly More Extended Conformational Space in Solution Compared with the Crystalline State

The SAXS measurement yields structural parameters on the time and ensemble average of a protein in solution, providing that the solution is free of aggregation and interparticle interference effects. At protein concentrations of $<2 \mathrm{mg} \mathrm{ml}^{-1}(65 \mu \mathrm{M})$ and at $30^{\circ} \mathrm{C}$, monodisperse solutions of the MexR-wt and MexR$\mathrm{R} 21 \mathrm{~W}$ as pure homodimers were obtained (Figure 4 and Table $\mathrm{S} 1)$. In brief, characteristics of the data that in combination demonstrate the solutions are monodisperse homodimers free of interparticle interference effects include: log-log plots of $I(q)$ 
A

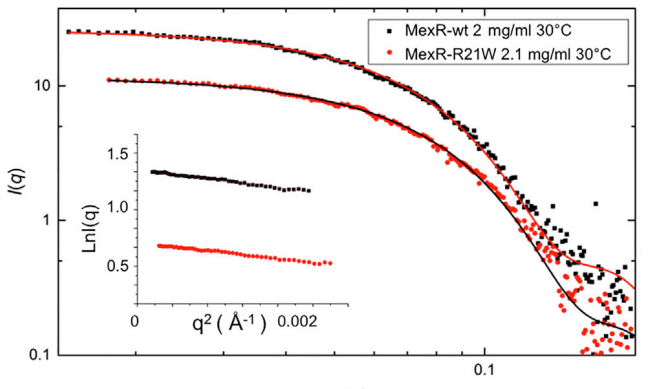

$q\left(\AA^{-1}\right)$

\section{B}

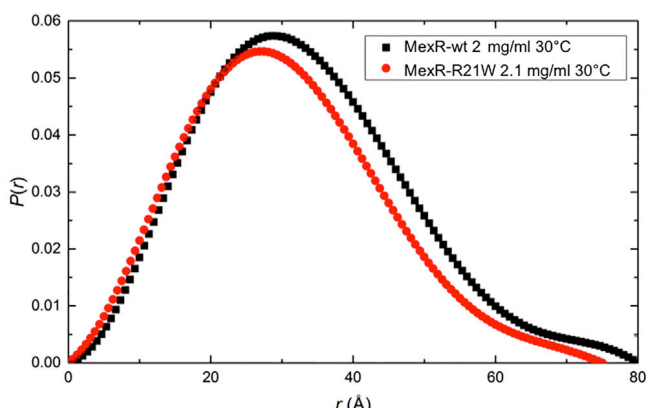

$r(\AA)$

Figure 4. Solution Analysis of MexR-wt and MexR-R21W Using SAXS

(A) Log /(q) versus $q$ for both MexR-wt (black squares) and MexR-R21W (red circles) scattering experimental data are shown together with the theoretica curves calculated form their crystal structure. Guinier plots are linear for $q R_{g}$ 1.3 as expected for monodisperse samples, and the molecular weight calculations correspond to dimer (see Table S1).

(B) Overlaid pair-distributions, $P(r)$ profiles for MexR-wt and MexR-R21W. See Experimental Procedures for full description.

versus $q$ flatten to zero slope at low $q$; Guinier plots are linear for values of $q R_{\mathrm{g}}<1.3 ; R_{\mathrm{g}}$ values from Guinier and $P(r)$ analyses are the same within error; and molecular weight estimates from $I(0)$ are within $10 \%-15 \%$ of what is expected for the homodimer. Table S1 provides complete details of SAXS data acquisition, reduction to $I(q)$ versus $q$, samples measured, and analysis programs used.

To compare the SAXS-derived parameters with what is expected from the crystal structures, we built models that included the residues missing from the crystal structures due to disorder (see Experimental Procedures). The slightly larger size of the MexR-wt construct is reflected in the larger $R_{\mathrm{g}}$ values (by 0.8 $1.2 \AA$ ) for the MexR-wt model homodimers (Table S1). Importantly, SAXS results consistently reveal experimentally derived $R_{\mathrm{g}}$ values for both MexR-wt and MexR-R21W that are significantly larger than the models (by $\sim 3 \AA$ for MexR-wt and $\sim 2 \AA$ for MexR-R21W), and a somewhat more extended $P(r)$. This in- dicates that both proteins in solution sample a wider conformational space than the crystal-based models, in agreement with the MD simulations. Furthermore, the increase in $R_{\mathrm{g}}$ is significantly larger for the wild-type compared with the mutant, which is in qualitative agreement with the wild-type protein occupying the more extended conformations. While the overall agreement between $I(q)$ versus $q$ for the crystal structure-based models versus experiment looks reasonable by eye (Figure 4), the $\chi$ values are high (1.67 and 1.49 for MexR-wt and MexR$\mathrm{R} 21 \mathrm{~W}$ ) due to the significant deviations in the low $q$ regime, as reflected in the larger $R_{\mathrm{g}}$ values, which is most sensitive to the longest dimensions in the scattering particle. Together, this is in agreement with a structurally extended ensemble in solution compared with that given by the crystal structures.

\section{DISCUSSION}

In this work we present a high-resolution structure of the MDR mutant MexR-R21W, which closely resembles previously obtained structures of MexR-wt. Our MD studies show that over the course of the simulation, the MexR-wt conformational ensemble is significantly extended compared with that of MexR-R21W. Importantly, the ensemble of structures visited by MexR-wt during the MD simulation includes structures with close resemblance to the DNA-bound state for MexR-wt, which is only intermittently visited by MexR-R21W. Independently, our experimental SAXS data indicate that the homodimers from the MexR-wt and MexR-R21W crystal structures provide models that reasonably represent the solution state, but that both proteins occupy a significantly more extended conformational space in solution than in in the crystalline state, with the MexRwt form showing evidence for greater extension than the mutant when comparing relative increases in $R_{\mathrm{g}}$ values compared with the crystal structure-based models. Based on joint assessment of all available data, we therefore propose that MexR-R21W mutation induces a population shift resulting in a restriction of the conformational ensemble to predominantly include conformations that are less likely to bind DNA. This restriction in MexR$\mathrm{R} 21 \mathrm{~W}$ leads to a significant compaction of the conformational ensemble relative to MexR-wt that is observed both in silico in MD, and experimentally in solution by SAXS analysis. The limited NMR data obtainable agree with this view and, by increased line broadening, support more extensive conformational exchange for MexR-wt compared with MexR-R21W (Figure S1).

Our high-resolution structure of MexR-R21W shows how aromatic ring structures can be accommodated in the interdomain cavity, which is a conserved feature in the ligand-responsive MarR family (Chang et al., 2010; Davis et al., 2013; Saridakis et al., 2008). Indeed, the ArmR protein, which inhibits MexR DNA binding, occupies the same interdomain cavity (Wilke et al., 2008), and native phenol-like inducer molecules were proposed to occupy the same space (Starr et al., 2012). Notably, in the MexR-ArmR complex, Arg21 forms key interactions with the ArmR peptide (Wilke et al., 2008) that is interrupted by the R21W mutation (Figure S6). Thus, it is highly likely that the mutation of Arg21 to Trp residue would also negatively affect ArmR binding to MexR.

Given that the MexR-R21W variant mimics the allosterically induced derepressed state of MexR, our finding contrasts with 


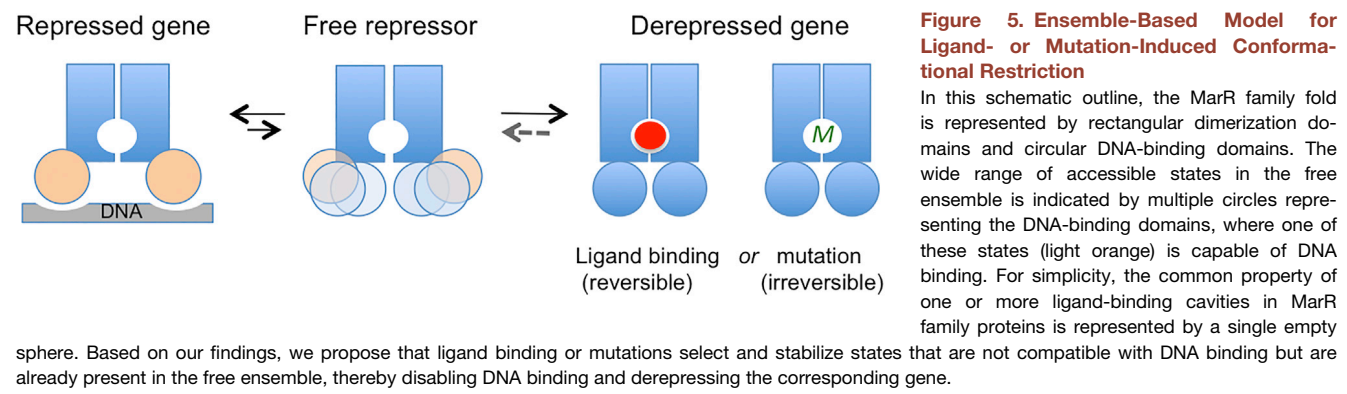

the currently prevailing model of allosteric regulation in the MarR family (Birukou et al., 2014; Hong et al., 2005). In the prevailing model, it is proposed that small-molecule binding to sites distant from the DNA-binding interface, or allosteric mutations, effectuate derepression by consecutive structural displacements of DNA-binding recognition elements and subdomain rotations originating from the allosteric binding site (Birukou et al., 2014; Hong et al., 2005). Since MexR-wt binds DNA with nM affinity whereas MexR-R21W is devoid of DNA binding (Andrésen et al., 2010), this model would predict an effector-bound conformation that differs significantly from previously observed wildtype conformations. We were therefore surprised to find that the structure of MexR-R21W closely resembles conformations within the wild-type apo MexR ensemble. Furthermore, our MD simulations and SAXS data jointly suggest that the MexR apo ensemble covers an even more extensive structural space than is presented by the crystal structures. These observations make it unlikely that the disrupted DNA binding in the MDR mutant is based on rigid structural differences.

To understand the relevance of our finding, we need to relate the structure-function relationships within the MarR family to recent key studies of allosterically governed interactions that display no structural change (Nussinov and Tsai, 2015). In particular, recent discoveries in CAP protein allostery provide a new perspective on allosteric regulation in the MarR system. Activation of gene expression in CAP repressor proteins is allosterically activated by cyclic AMP binding to a distant site, which results in a reorientation of the DNA-binding helices compatible with DNA binding to specific sites, which in turn recruits polymerase and effectuates gene expression (Popovych et al., 2009). However, in a mutant CAP* $^{*}$ (T127L, S128L), which binds DNA despite its ground state adopting a conformation incompatible with binding, DNA binding is inhibited rather than activated by cyclic guanosine monophosphate (cGMP) (Tzeng and Kalodimos, 2013). Careful analysis of this observation reveals that in the absence of cGMP, the CAP* protein transiently occupies a DNA-binding capable state; binding of cGMP has no structural effect on the ground-state structure but depletes access to the DNA-binding-capable conformation.

Our results suggest a regulatory pathway for MexR whereby both allosteric effector molecules and allosteric mutants may stabilize a non-DNA-binding ground state, similar to that observed for CAP* (Tzeng and Kalodimos, 2013). To be biologically efficient, apo MarR family repressor proteins must simultaneously be capable both of specific DNA binding and of binding a wide

range of effector molecules such as aromatic compounds and antibiotics, but with distinct structural outcomes (Figure 5). To accomplish this task requires a mechanism that enables less specific ligand binding to effectuate a highly efficient response. The advantage of an allosteric mechanism that by stabilizing the ground state blocks transient access to the DNA-binding state is that small-molecule binding essentially in any groove or cavity of the ground state would effect such stabilization. This would provide the required versatility in effector ligand binding by releasing any requirements for highly specific binding with concomitantly stringent consecutive structural changes. Notably, a common property of the MarR family is the presence of sizable grooves and cavities, thus featuring highly porous structures (Chang et al., 2010). Furthermore, recent mutational analysis has identified regulatory ligand-binding pockets in the interdomain interface of MarR family proteins, distant from the DNA-binding surface, supporting the important role of such sites in allosteric regulation (Duval et al., 2013; Gupta and Grove, 2014).

Due to the functional requirement of efficient and diverse ligand binding for many members of the MarR family, we propose that the allosterically effected population shifts that we here describe for MexR (Figure 5) may indeed be common also to other members of the MarR family. The extensive interdomain spread that we observe for MexR in its free-state ensemble not only enables swift binding of regulatory effectors, but also ensures direct access to conformations that are compatible with DNA binding, with disulfide formation in response to stressinduced oxidation (Brugarolas et al., 2012; Chen et al., 2008; Hao et al., 2014) and with binding of more complex ligands such as ArmR (Wilke et al., 2008). While cryo-crystallography selects for single conformations and is not optimal for the analysis of ensembles, multiple conformations of the same free protein have indeed been observed in the crystal structures of both MexR (Lim et al., 2002) and MepR (Birukou et al., 2013; Figure S2B). Ligand binding, or oxidation, does not then by itself confer structural change, but rather acts as a stabilizer or rigidifier of a substate of the conformational ensemble. Based on our evaluation of allosteric contact networks and how these are collapsed by the ligand-binding mimicking R21W mutation (Figure 3 and Table S2), we propose that independent motion in the free state of dimerization- and DNA-binding domains may be an essential component also for other MarR family proteins, since it enables partial occupancy of a wide ensemble of biologically relevant binding modes.

1318 Structure 24, 1311-1321, August 2, 2016 
Taken together, our computational results and experimenta data reveal that while the MexR-R21W and MexR-wt crysta structures are highly similar, their conformational dynamics in solution differ; the ensemble of conformations describing the MexR-R21W predominantly comprises selected conformations from the MexR-wt ensemble with low similarity to DNA-bound structures, thus presenting an allosteric regulation model that relies on population shift. The allosteric model for an MarR family protein presented here significantly extends our functional understanding of both ligand-triggered responses and MDR mutations effecting derepression of efflux pump protein expression.

\section{EXPERIMENTAL PROCEDURES}

Cloning, Protein Expression, and Purification

The sequence encoding the mexR open reading frame (residues Val5 to Leu139) with the mutation R21W was subcloned into the pNIC28-Bsa4 vector, with $6 \mathrm{x}$-His tag in 22-amino-acid $\mathrm{N}$-terminal fusion peptide, with tobacco etch virus (TEV) protease cleavage site. Transformed BL21(DE3) were grown a $37^{\circ} \mathrm{C}$ until $\mathrm{OD}_{600}$ of 2.0 , induced by $1 \mathrm{mM}$ isopropyl $\beta$-D-1-thiogalactopyrano$37^{\circ} \mathrm{C}$ until $\mathrm{OD}_{600}$ of 2.0 , induced by $1 \mathrm{mM}$ isopropyl $\beta$-D-1-thiogalactopyrano-
side, harvested, and lysed by sonication in lysis buffer ( $300 \mathrm{mM}$ sodium side, harvested, and lysed by sonication in lysis buffer ( $300 \mathrm{mM}$ sodium
chloride, $20 \mathrm{mM}$ Tris [pH 8.0], $20 \mathrm{mM}$ imidazole, $1 \mathrm{mM}$ tris(2-carboxyethyl) phosphine (TCEP), $10 \%$ glycerol, $1 \mathrm{mg} / \mathrm{ml}$ lysozyme, $5 \mathrm{U} / \mathrm{ml}$ DNase I, an $1 \times$ Protease Inhibitor tablets (Roche Diagnostics), and purified on Ni-nitrilotriacetic acid beads at $4^{\circ} \mathrm{C}$. Where required for SAXS, the $6 \mathrm{x}$-His tag was cleaved on the column. The flow-through was purified on a Superdex-75 colum and SDS-PAGE. Purified protein was concentrated using an Amicon ultra concentrator (Millipore), flash-frozen in $\mathrm{N}_{2}(h)$ and stored at $-80^{\circ} \mathrm{C}$, unt further use. MexR-wt (residues Met1 to lle147) was produced using the same protocol with added heparin-Sepharose purification (Andrésen et al., 2010). Biophysical analysis of the MexR-wt $t_{5-139}$ construct was prevented due to its very low yield in Escherichia coli overexpression, possibly due to altered $\mathrm{p} /$ on removal of acidic disordered tails ( +0.4 units), thus bringing the protein into the edge of an unfavorable region for proteins expressed in E. coli (Han and Lee, 2006).

X-Ray Structure Determination and Analysis

Screening for crystallization conditions was performed in 96-well sitting-drop plates at $4^{\circ} \mathrm{C}$ and $20^{\circ} \mathrm{C}$ at a concentration of $21 \mathrm{mg} / \mathrm{ml}$. An iterative seeding approach, using micro- and macroseeding (D'Arcy et al., 2014) from spherulite-like and thin crystals, gave a single large cylindrical crystal from $0.2 \mathrm{M}$ Trimethylamine $\mathrm{n}$-oxide, $20 \%$ polyethylene glycol MME 2000, and $0.1 \mathrm{M}$ Tris (pH 8.5), which was soaked in mother liquor contaning $10 \%$ glycerol prior to freezing in $\mathrm{N}_{2}($ ). MexR R21W diffraction data were collected at the Diamond
Light Source i24 beamline. Only the His tag MexR R21W crystals diffracted Light Source 24 beamline. Only the His tag MexR R21W crystals diffracted
well, likely favored by crystal contacts involving the TEV cleavage site Tyr. A native dataset was collected to $2.19 \AA$ resolution, processed using XDS (Kabsch, 2010) and using PHASER (McCoy et al., 2007) to find the solution by molecular replacement. The protein was built using Coot (Emsley et al., 2010), refined using BUSTER (Blanc et al., 2004), and validated with MolProbity (Chen etat. 2010b) (Bcor of 1.14 (Table1). The degree of asym MolProbity (Che degree of asymmechas evaly corresponds to Sdev $=0$ and $\mathrm{Sdev}>0$ denotes asymmetry to varying degrees (André et al., 2008). All protein representations were generated with PyMOL Molecular Graphics System, Version 1.2r3pre (Schrödinger).

mall-Angle X-Ray Scattering Measurements

SAXS data for MexR-wt and MexR-R21W were collected at $30^{\circ} \mathrm{C}$ in $50 \mathrm{mM}$ 2-( $\mathrm{N}$-morpholino)ethanesulfonic acid, $150 \mathrm{mM} \mathrm{NaCl}$, and $1 \mathrm{mM}$ TCEP (pH 6.0), using an Anton Paar SAXSess with line geometry and a sealed-tube $X$-ray source (CuK $\alpha$ wavelength of $1.54 \AA$ ) coupled to a CCD camera. Scattering data were acquired over a $q$ range $0.01-0.4 \AA^{-1}$, where $q=\frac{4 \pi \sin \theta}{\lambda}$. Data were placed on an absolute scale using the known scattering from pure water and reduced to $I(q)$ versus $q$ for the protein by subtraction of the solvent blank.
Data reduction was done using SAXSquant (Anton Paar). Molecular weight calculations from I(0) values were made as described by Orthaber and Glatter 2000), and values for partial specific volume, contrast, and expected molecular weight were calculated using MULCh (Whitten et al., 2008). Guinier and $P(r)$ analyses and model fitting to the scattering data were performed using the ATSAS proged using the coordinate les PDB: 1LNW (wild-type dimer chain CD) and 4ZZL (mutant dimer this study). Modeling of residues with missing electron density in the dimer structures used symmetry-based modeling of residues that presented electron density in the other chain; MexR-wt, residues $G(-1), S(0)$, M1, and L139-1147, or in a related dimer (MexR-wt, residues L139-1147 modeled from PDB: 1LNW, chains AB), or built de novo (MexR-R21W, residues $G(-1)$ and $\operatorname{Ser}(0)$ ) or modeled onto very weak density not included in the see Table S1.

\section{Molecular Dynamics}

MexR-wt (PDB: 1LNW, chains CD, EF, and GH) and MexR-R21W (PDB: 4ZZL), selecting MexR residues 5-139 for all proteins for consistency, were used as starting structures in MD simulations using Gromacs 4.5.5 (Pronk et al., 2013) with Amber99sb force field and 2-fs time steps. Electrostatic interactions were evaluated using particle mesh Ewald summation every step. A $13 \AA ̊$ cutoff was used for electrostatics and van der Waals interactions with neighbor lists was used for electrostatics and van der Waals interactions with neighbor lists
updated every five steps. Simulations were performed at 300 K by using a Bussi
thermostat (Bussi et al., 2007). Pressure coupling was achieved with a Parrithermostat (Bussi et al., 2007). Pressure coupling was achieved with a Parri-
nello-Rahman barostat (Parrinello, 1981) with a time constant of 5 ps and a compressibility of $4.510^{-5} \mathrm{bar}^{-1}$. Each starting structure was energy minimized using steepest descent followed by $0.5 \mathrm{~ns} \mathrm{MD}$ with positional restraints on the protein using the SHAKE algorithm (Ryckaert et al., 1977) followed by 0.5 ns with weaker (11 was relaxed without any restraints for 2 ns before data were collected for $100 \mathrm{~s}$. This was repeated ten times with different randomized starting velocities for each of the four different starting conformations, totaling in $4 \mu \mathrm{s}(10 \times 4 \times$ $100 \mathrm{~ns})$ simulation time. Trajectories were clustered with g_cluster, using the gromos method with $3 \AA \AA$ RMSD cutoff. Projections on the principal components chosen from the essential dynamics analysis (Bakan et al., 2011) (see below) was used to visualize the clusters on the same coordinate plane as the MexR-wt and MexR-R21W conformational distributions. Ensemble convergence was assessed by clustering the complete set of trajectories, and gence was assessed by clus clusters as the full set of trajectories (Lyman and Zuckerman, 2006). $10 \times$ $4 \times 50$-ns subtrajectories, corresponding to half of the total simulation time, were sufficient to populate the largest clusters, containing $90 \%$ of the structures, in equal proportion as the complete set of trajectories.

Analysis of Allosteric Communities

Calculation of the probabilistic graph model and subsequent community analysis of MexR-wt and MexR-R21W trajectories, pooled separately, was done with the Protein Structure Network module in the program Wordom (Seeber et al., 2011). Termini parameters and distance, stable, and hub contact cutof parameters were set to $0,4.5,0.5$, and $3.0 \AA$, respectively. An interaction minimum strength of 3.0 described the observed MexR-wt and MexR-R21W community characteristics equally well.

Essential Dynamics Analysis

The essential dynamics analysis method of the ProDy (Bakan et al., 2011) Python package was used to perform the principal component analysis of the trajectories, where all trajectories (MexR-wt and MexR-R21W) were pooled and superimposed on $\mathrm{C}_{\alpha}$ atoms of the dimer core (residues 5-17 and 116-138). The two largest eigenvectors were extracted from this covariance matrix with the distribution of projections over the vectors visualized separately for MexRwt and MexR-R21W, smoothed with a Gaussian filter with sigma 2.0 using the SciPy python package (Humphis ized to unity compared using a similar analysis but separately for each set of starting structures. The two largest principal components were visualized by NMWiz in VMD (Humphrey et al., 1996) as directional arrows centered at $\mathrm{C}_{\alpha}$ atoms of the representative structures projecting closest to zero on both components. 
ACCESSION NUMBERS

Coordinates and structure factors for MexR-R21W structure described here have been deposited in the PDB with accession code PDB: 4ZZL.

\section{SUPPLEMENTAL INFORMATION}

Supplemental Information includes six figures, two tables, and one 3D molecular model and can be found with this article online at $\mathrm{http}: / / \mathrm{dx}$.doi.org/10. 1016/.str.2016.06.008

\section{AUTHOR CONTRIBUTIONS}

Designed research: B.W. and M.S. Performed research: M.A., R.P., C.A., and B.W. Analyzed data: M.A., R.P., J.T., M.M., B.W., and M.S. Wrote the paper: M.A., R.P., J.T. B.W., and M.S.

\section{ACKNOWLEDGMENTS}

Dr. Jonas Carlsson Almlöf and Javed M.E. Ziauddin are acknowledged for their contributions during the initiation of this project. We thank Dr. Don Parkin for technical assistance in obtaining the SAXS data. We acknowledge the Protein Science Facility (PSF) at Karolinska Institutet (psf.ki.se) for access to protein crystallography resources, and Dr. Tien-Chye Tan and Dr. Christian Löw for crystallography resources, and Dr. Th Chye Tan and Dr. Christian Low for collecting $X$ (Harwell, UK). PSF acknowledges funding from the European Community's Seventh Framework Program (FP7/2007-2013) under BioStruct-X (gran agreement No. 283570), Swedish e-Science Research Center, and the Swedish Research Council. The MD simulations were performed on resources provided by the Swedish National Infrastructure for Computing (SNIC) and the Swedish e-Science Research Center at the National Supercomputer Center (NSC) in Linköping. The Swedish Research Council supported this work by project grants to M.S. and B.W., and by a Tage Erander Visiting Professor project grant to J.T.

\section{Received: March 28, 2016}

Revised: May 20, 2016

Accepted: June 5, 2016

Published: July 14, 2016

\section{REFERENCES}

Adewoye, L., Sutherland, A., Srikumar, R., and Poole, K. (2002). The mexR repressor of the mexAB-oprM multidrug efflux operon in Pseudomonas aeruginosa: characterization of mutations compromising activity. J. Bacteriol. 184 4308-4312.

Alekshun, M.N., Kim, Y.S., and Levy, S.B. (2000). Mutational analysis of MarR, the negative regulator of marRAB expression in Escherichia coli, suggests the presence of two regions required for DNA binding. Mol. Microbiol. 35, 13941404.

Alekshun, M.N., Levy, S.B., Mealy, T.R., Seaton, B.A., and Head, J.F. (2001). The crystal structure of MarR, a regulator of multiple antibiotic resistance, at 2.3 A resolution. Nat. Struct. Biol. 8, 710-714.

André, I., Strauss, C.E.M., Kaplan, D.B., Bradley, P., and Baker, D. (2008). Emergence of symmetry in homooligomeric biological assemblies. Proc Natl. Acad. Sci. USA 105, 16148-16152.

Andrésen, C., Jalal, S., Aili, D., Wang, Y., Islam, S., Jarl, A., Liedberg, B., Wretlind, B., Mårtensson, L.-G., and Sunnerhagen, M. (2010). Critical biophysical properties in the Pseudomonas aeruginosa efflux gene regulator MexR are targeted by mutations conferring multidrug resistance. Protein Sci. 19 680-692.

Bakan, A., Meireles, L.M., and Bahar, I. (2011). ProDy: protein dynamics inferred from theory and experiments. Bioinformatics 27, 1575-1577.

Blanc, E., Roversi, P., Vonrhein, C., Flensburg, C., Lea, S.M., and Bricogne, G. (2004). Refinement of severely incomplete structures with maximum likelihood in BUSTER-TNT. Acta Crystallogr. D Biol. Crystallogr. 60, 2210-2221.
Biedermannova, L., E. Riley, K., Berka, K., Hobza, P., and Vondrasek, J. (2008). Another role of proline: stabilization interactions in proteins and protein complexes concerning proline and tryptophane. Phys. Chem. Chem. Phys. 10, 6350.

Birukou, I., Tonthat, N.K., Seo, S.M., Schindler, B.D., Kaatz, G.W., and Brennan, R.G. (2013). The molecular mechanisms of allosteric mutations impairing MepR repressor function in multidrug-resistant strains of Staphylococcus aureus. $\mathrm{mBio} 4$, e00528-e00613.

Birukou, I., Seo, S.M., Schindler, B.D., Kaatz, G.W., and Brennan, R.G. (2014). Structural mechanism of transcription regulation of the Staphylococcus aureus multidrug efflux operon mepRA by the MarR family repressor MepR. Nucleic Acids Res. 42, 2774-2788.

Brugarolas, P., Movahedzadeh, F., Wang, Y., Zhang, N., Bartek, I.L., Gao, Y.N., Voskuil, M.I., Franzblau, S.G., and He, C. (2012). The oxidationsensing regulator (MosR) is a new redox-dependent transcription factor in sensing regulator (MosR) is a new redox-dependent transcripton

Bussi, G., Donadio, D., and Parrinello, M. (2007). Canonical sampling through velocity rescaling. J. Chem. Phys. 126, 014101.

Cao, L., Srikumar, R., and Poole, K. (2004). MexAB-OprM hyperexpression in NalC-type multidrug-resistant Pseudomonas aeruginosa: identification and characterization of the nalC gene encoding a repressor of PA3720-PA3719. Mol. Microbiol. 53, 1423-1436.

Chang, Y.-M., Jeng, W.-Y., Ko, T.-P., Yeh, Y.-J., Chen, C.K.-M., and Wang, A.H.-J. (2010). Structural study of TcaR and its complexes with multiple antibiotics from Staphylococcus epidermidis. Proc. Natl. Acad. Sci. USA 107 8617-8622.

Chen, H., Hu, J., Chen, P.R., Lan, L., Li, Z., Hicks, L.M., Dinner, A.R., and He, C. (2008). The Pseudomonas aeruginosa multidrug efflux regulator MexR uses an oxidation-sensing mechanism. Proc. Natl. Acad. Sci. USA 105, 13586-13591. Chen, H., Yi, C., Zhang, J., Zhang, W., Ge, Z., Yang, C.-G., and He, C. (2010a). Structural insight into the oxidation-sensing mechanism of the antibiotic resistance of regulator MexR. EMBO Rep. 11, 685-690.

Chen, V.B., Arendall, W.B., Headd, J.J., Keedy, D.A., Immormino, R.M. Kapral, G.J. Murray, LW. Richardson, J.S., and Richardson, D.C. (2010b). MolProbity: all-atom structure validation for macromolecular crystallography. Acta Crystallogr. D Biol. Crystallogr. 66, 12-21.

D'Arcy, A., Bergfors, T., Cowan-Jacob, S.W., and Marsh, M. (2014). Microseed matrix screening for optimization in protein crystallization: what have we learned? Acta Crystallogr. F Struct. Biol. Commun. 70, 1117-1126.

Davis, J.R., Brown, B.L., Page, R., and Sello, J.K. (2013). Study of PcaV from Streptomyces coelicolor yields new insights into ligand-responsive MarR family transcription factors. Nucleic Acids Res. 41, 3888-3900.

Dolan, K.T., Duguid, E.M., and He, C. (2011). Crystal structures of SlyA protein, a master virulence regulator of salmonella, in free and DNA-bound states. J. Biol. Chem. 286, 22178-22185.

Duval, V., McMurry, L.M., Foster, K., Head, J.F., and Levy, S.B. (2013). Mutational analysis of the multiple-antibiotic resistance regulator MarR reveals a ligand binding pocket at the interface between the dimerization and DNA binding domains. J. Bacteriol. 195, 3341-3351.

Emsley, P., Lohkamp, B., Scott, W.G., and Cowtan, K. (2010). Features and development of coot. Acta Crystallogr. D Biol. Crystallogr. 66, 486-501.

Grove, A. (2013). MarR family transcription factors. Curr. Biol. 23, R142-R143. Guerra, A.J., Dann, C.E., and Giedroc, D.P. (2011). Crystal structure of the zinc-dependent MarR family transcriptional regulator AdcR in the Zn(II)-bound state. J. Am. Chem. Soc. 133, 19614-19617.

Gupta, A., and Grove, A. (2014). Ligand-binding pocket bridges DNA-binding and dimerization domains of the urate-responsive MarR homologue MftR from Burkholderia thailandensis. Biochemistry 53, 4368-4380.

Hao, Z., Lou, H., Zhu, R., Zhu, J., Zhang, D., Zhao, B.S., Zeng, S., Chen, X., Chan, J., He, C., et al. (2014). The multiple antibiotic resistance regulator MarR is a copper sensor in Escherichia coli. Nat. Chem. Biol. 10, 21-28.

Han, M.J., and Lee, S.Y. (2006). The Escherichia coli proteome: past, present and future prospects. Microbiol. Mol. Biol. Rev. 70, 362-439.

1320 Structure 24, 1311-1321, August 2, 2016 
Hong, M., Fuangthong, M., Helmann, J.D., and Brennan, R.G. (2005). Structure of an OhrR-ohrA operator complex reveals the DNA binding mechanism of the MarR family. Mol. Cell 20, 131-141.

Humphrey, W., Dalke, A., and Schulten, K. (1996). VMD: visual molecular dynamics. J. Mol. Graph 14, 33-38, 27-28.

Jalal, S., Wretlind, G., Gotoh, N., and Wretlind, B. (1999). Rapid identification o mutations in a multidrug efflux pump in Pseudomonas aeruginosa. APMIS 107 1109-1116.

Jones, E., Oliphant, T.E., and Peterson, P. (2001). SciPy: Open Source Scientific Tools for Python [Computer Software] www.scipy.org. Kabsch, W. (2010). XDS. Acta Crystallogr. D Biol. Crystallogr. 66, 125-132. Kumarevel, T., Tanaka, T., Umehara, T., and Yokoyama, S. (2009). ST1710DNA complex crystal structure reveals the DNA binding mechanism of the MarR family of regulators. Nucleic Acids Res. 37, 4723-4735.

Li, X.Z., Zhang, L., Srikumar, R., and Poole, K. (1998). Beta-lactamase inhibitors are substrates for the multidrug efflux pumps of Pseudomonas aeruginosa. Antimicrob. Agents Chemother. 42, 399-403.

Lim, D., Poole, K., and Strynadka, N.C.J. (2002). Crystal structure of the MexR repressor of the mexRAB-oprM multidrug efflux operon of Pseudomonas aeruginosa. J. Biol. Chem. 277, 29253-29259.

Lyman, E., and Zuckerman, D.M. (2006). Ensemble-based convergence analysis of biomolecular trajectories. Biophys. J. 91, 164-172.

McCoy, A.J., Grosse-Kunstleve, R.W., Adams, P.D., Winn, M.D., Storoni, L.C. and Read, R.J. (2007). Phaser crystallographic software. J. Appl. Crystallogr. 40, 658-674.

Nussinov, R., and Tsai, C.-J. (2015). Allostery without a conformationa change? Revisiting the paradigm. Curr. Opin. Struct. Biol. 30, 17-24.

Orthaber, D., and Glatter, O. (2000). Synthetic phospholipid analogs: a structural investigation with scattering methods. Chem. Phys. Lipids 107, 179-189. Parrinello, M. (1981). Polymorphic transitions in single crystals: a new molecular dynamics method. J. Appl. Phys. 52, 7182-7190.

Perera, I.C., and Grove, A. (2010). Molecular mechanisms of ligand-mediated attenuation of DNA binding by MarR family transcriptional regulators. J. Mol. Cell Biol. 2, 243-254.

Perera, I.C., Lee, Y.-H., Wilkinson, S.P., and Grove, A. (2009). Mechanism for attenuation of DNA binding by MarR family transcriptional regulators by smal molecule ligands. J. Mol. Biol. 390, 1019-1029.

Petoukhov, M.V., Franke, D., Shkumatov, A.V., Tria, G., Kikhney, A.G., Gajda, M., Gorba, C., Mertens, H.D.T., Konarev, P.V., and Svergun, D.I. (2012). New developments in the ATSAS program package for small-angle scattering data analysis. J. Appl. Crystallogr. 45, 342-350.

Poole, K. (2001). Multidrug efflux pumps and antimicrobial resistance in Pseudomonas aeruginosa and related organisms. J. Mol. Microbiol. Biotechnol. 3, 255-264.

Poole, K., Tetro, K., Zhao, Q., Neshat, S., Heinrichs, D.E., and Bianco, N. (1996). Expression of the multidrug resistance operon mexA-mexB-oprM in Pseudomonas aeruginosa: mexR encodes a regulator of operon expression. Antimicrob. Agents Chemother. 40, 2021-2028.
Popovych, N., Tzeng, S.-R., Tonelli, M., Ebright, R.H., and Kalodimos, C.G. 2009). Structural basis for cAMP-mediated allosteric control of the catabolite activator protein. Proc. Natl. Acad. Sci. USA 106, 6927-6932.

Pronk, S., Páll, S., Schulz, R., Larsson, P., Bjelkmar, P., Apostolov, R., Shirts, M.R., Smith, J.C., Kasson, P.M., van der Spoel, D., et al. (2013). GROMACS 4.5: a high-throughput and highly parallel open source molecular simulation toolkit. Bioinformatics 29, 845-854.

Radhakrishnan, A., Kumar, N., Wright, C.C., Chou, T.-H., Tringides, M.L. Bolla, J.R., Lei, H.-T., Rajashankar, K.R., Su, C.-C., Purdy, G.E., et al. (2014). Crystal structure of the transcriptional regulator Rv0678 of Mycobacterium tuberculosis. J. Biol. Chem. 289, 16526-16540.

Ryckaert, J.-P., Giovanni, C., and Berendsen, H.J.C. (1977). Numerical integration of the cartesian equations of motion of a system with constraints: molecular dynamics of n-alkanes. J. Comp. Phys. 23, 1-15.

Saridakis, V., Shahinas, D., Xu, X., and Christendat, D. (2008). Structural insight on the mechanism of regulation of the MarR family of proteins: high-resolution crystal structure of a transcriptional repressor from Methanobacterium thermoautotrophicum. J. Mol. Biol. 377, 655-667.

Seeber, M., Felline, A., Raimondi, F., Muff, S., Friedman, R., Rao, F., Caflisch, A., and Fanelli, F. (2011). Wordom: a user-friendly program for the analysis of molecular structures, trajectories, and free energy surfaces. J. Comput. Chem. 32, 1183-1194

Slutzki, M., Jobby, M.K., Chitayat, S., Karpol, A., Dassa, B., Barak, Y., Lamed, R. Smith, S.P., and Bayer, E.A. (2013). Intramolecular clasp of the cellulosomal Ruminococaus flave Ruminococcus flavefaciens ScaA dockerin module confers structural stability FEBS Open Bio. 3, 398-405.

Srikumar, R., Paul, C.J., and Poole, K. (2000). Influence of mutations in the mexR repressor gene on expression of the MexA-MexB-oprM multidrug efflux system of Pseudomonas aeruginosa. J. Bacteriol. 182, 1410-1414.

Starr, L.M., Fruci, M., and Poole, K. (2012). Pentachlorophenol induction of the Pseudomonas aeruginosa mexAB-oprM efflux operon: involvement of repressors NalC and MexR and the antirepressor ArmR. PLoS One 7, e32684.

Tzeng, S.-R., and Kalodimos, C.G. (2013). Allosteric inhibition through suppression of transient conformational states. Nat. Chem. Biol. 9, 462-465.

Whitten, A.E., Cai, S., and Trewhella, J. (2008). MULCh: modules for the analysis of small-angle neutron contrast variation data from biomolecular assemblies. J. Appl. Crystallogr. 41, 222-226.

Wilke, M.S., Heller, M., Creagh, A.L., Haynes, C.A., McIntosh, L.P., Poole, K., and Strynadka, N.C.J. (2008). The crystal structure of MexR from Pseudomonas aeruginosa in complex with its antirepressor ArmR. Proc Natl. Acad. Sci. USA 105, 14832-14837.

Wilkinson, S.P., and Grove, A. (2006). Ligand-responsive transcriptional regulation by members of the MarR family of winged helix proteins. Curr. Issues Mol. Biol. 8, 51-62.

Ziha-Zarifi, I., Llanes, C., Köhler, T., Pechere, J.C., and Plesiat, P. (1999). In vivo emergence of multidrug-resistant mutants of Pseudomonas aeruginosa overexpressing the active efflux system MexA-MexB-OprM. Antimicrob. Agents Chemother. 43, 287-291. 
Structure, Volume 24

\section{Supplemental Information}

Mutation-Induced Population Shift in the MexR

Conformational Ensemble Disengages DNA Binding:

A Novel Mechanism for MarR Family Derepression

Madhanagopal Anandapadamanaban, Robert Pilstål, Cecilia Andresen, Jill Trewhella, Martin Moche, Björn Wallner, and Maria Sunnerhagen 


\section{Figure S1}

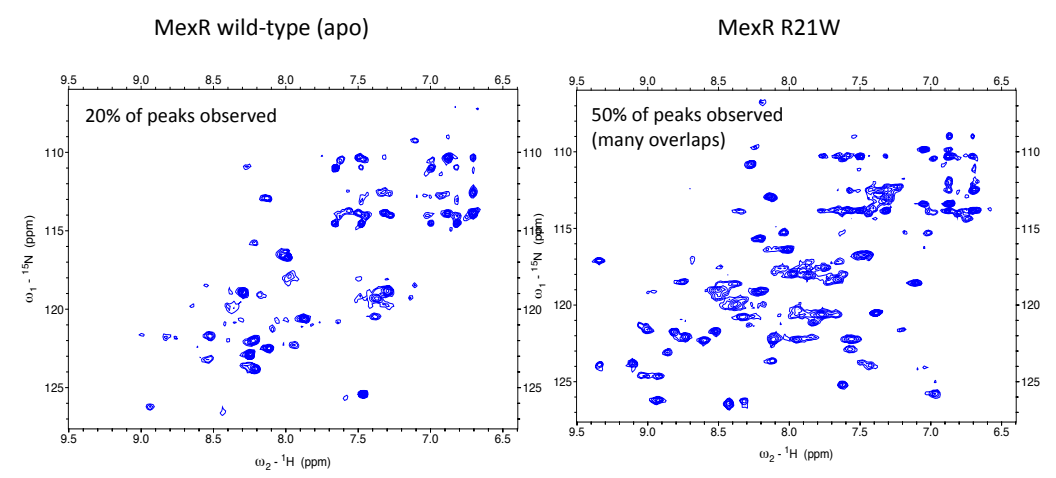

Figure S1, related to Figure 1 and 4. Solution analysis of MexR-Wt and -R21W using NMR. NMR studies of MexR-wt and MexR-R21W mutant. ${ }^{15} \mathrm{~N}$ TROSY spectra of MexR-wt and $-\mathrm{R} 21 \mathrm{~W}$ are shown. The observed signals as peaks in percentage of expected is annotated. While the sample condition is identical to that where SAXS measurements (see Methods), the MexR-R21W spectrum is more resolved in comparison to MexR-wt. ${ }^{15} \mathrm{~N}$-TROSY based HSQC experiments were collected at $30^{\circ} \mathrm{C}$ on a Varian 600 INOVA spectrometer at a concentration of $60 \mathrm{mM}$ (dimer) for both MexR-wt and -R21W, requiring four hours each. Final NMR samples were prepared in 50mM MES-monohydrate, $\mathrm{pH} 6.0,150 \mathrm{mM} \mathrm{NaCl}$ and $1 \mathrm{mM}$ TCEP, where the proteins were found by SAXS to be pure dimers at $60 \mu \mathrm{M}$. Despite extensive assay of buffer conditions, sample concentrations and temperatures by NMR and analytical gel filtration, no alternative sample condition was found to further improve NMR spectral quality. 


\section{Figure S2}
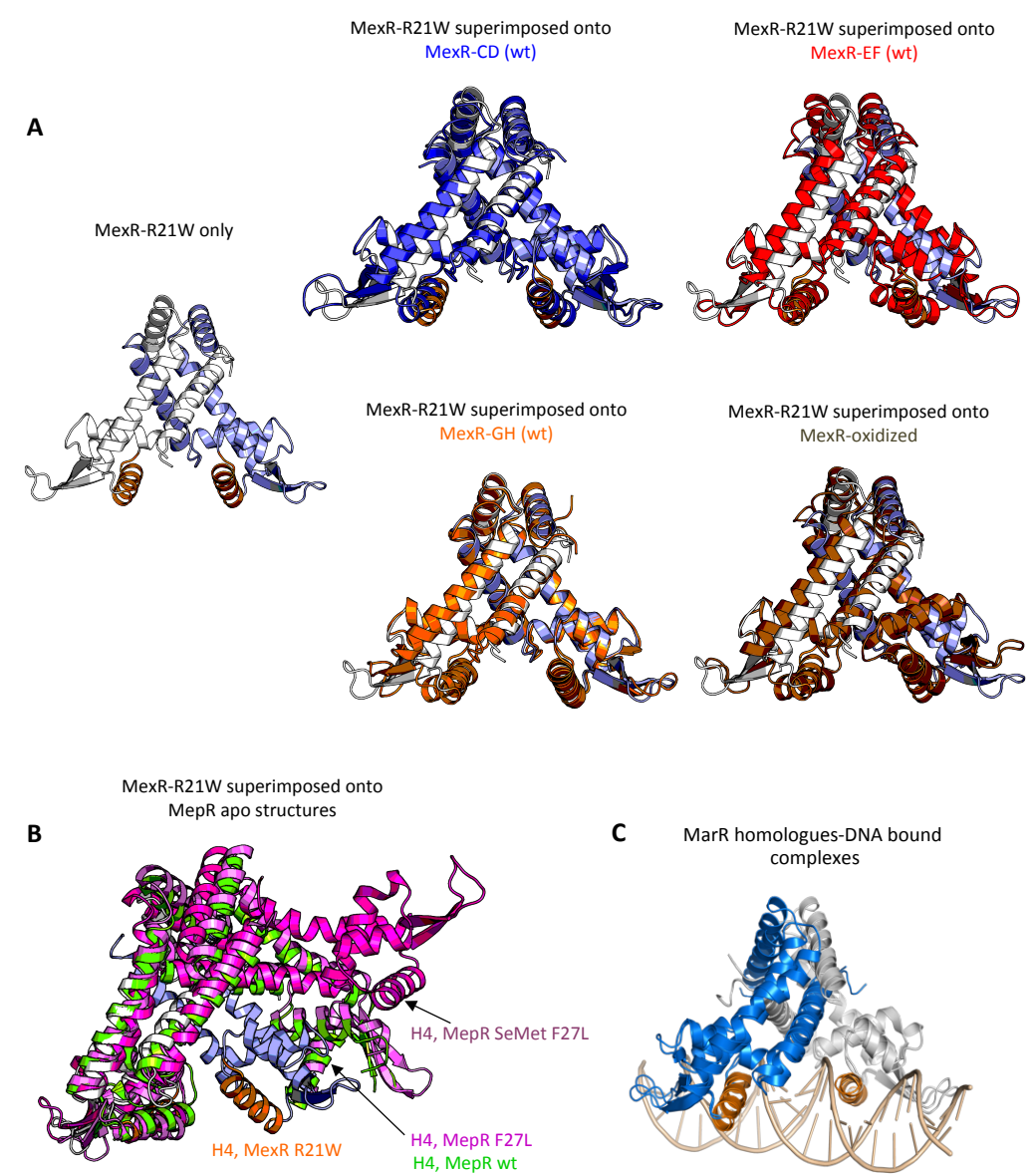

C MarR homologues-DNA bound complexes

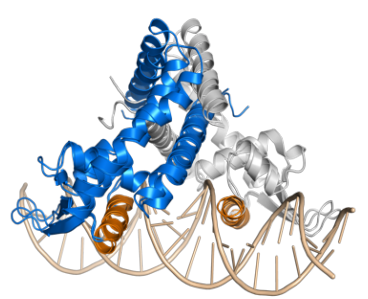

Figure S2, related to Figure 1. (A) Structural comparisons of MexR-R21W with other MexR-wt dimer chains (1LNW, and colored by chain CD in blue, EF in red and $\mathrm{GH}$ in orange) and MexR-oxidized structure (3MEX, in brown). (B) Structural superimposition of MexR-R21W structure to MarR homologue MepR apo structures (PDB codes below) through structural superimpositions in all $\mathrm{C} \alpha$ atoms. For clarity, MepR (wt and F27L) residues 49-58 are removed. PDB codes -MepR-wt (4L9J) and MepR-F27L mutant (4L9T, 4L9V). (C) Cartoon representation of MarR homologue DNA bound complexes, includes OhrR-DNA (1Z9C) and MepR-DNA (4LLN), represents for the DNA-bound conformations. 


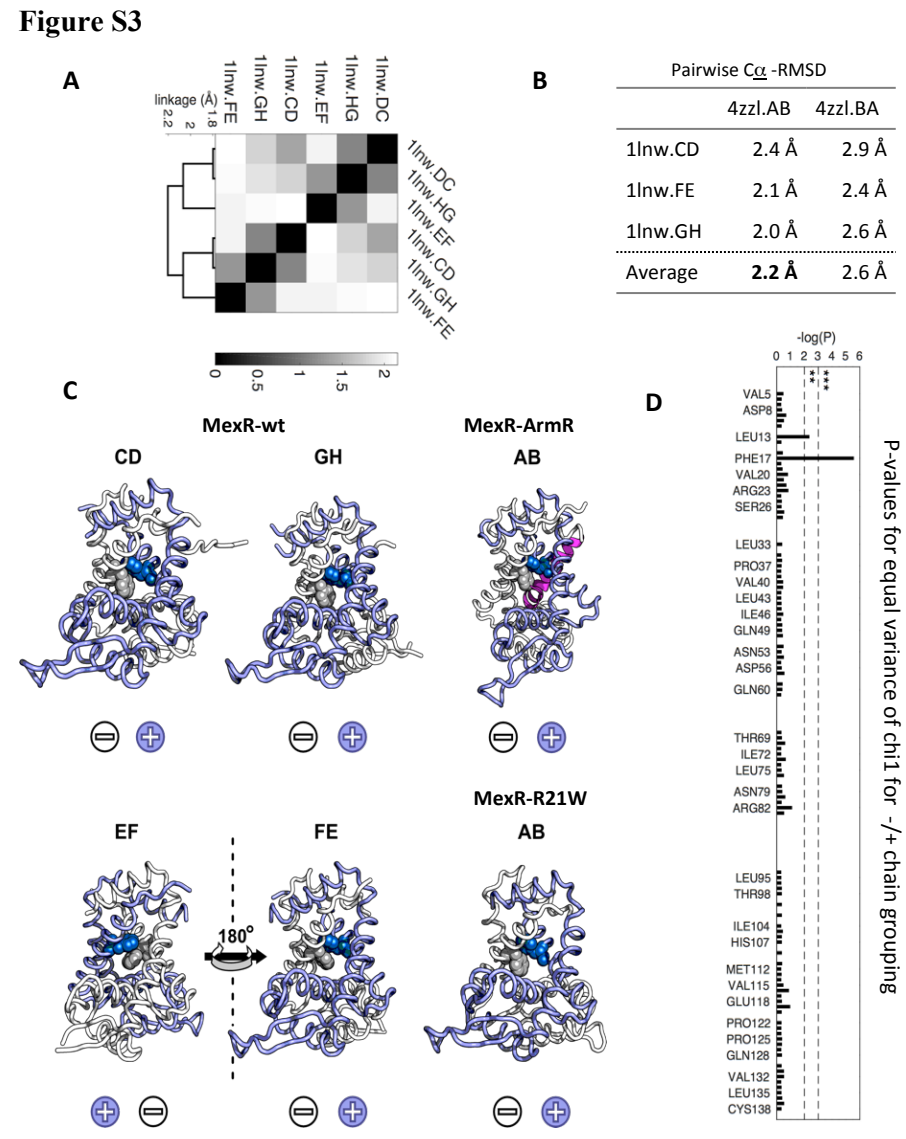

Figure S3, related to Figure 1, 2 and 3. (A) Dendrogram from single-linkage clustering on the pairwise RMSD of different chain ordering for the MexR-wt structures show a clustering in either DC, HG, EF or the equivalent CD, FE, GH chain order. (B) Pairwise Ca RMSD to the selected chain order for wt against the two possible chain orderings in R21W; showing that the $\mathrm{AB}$ ordering is more similar to the wt selected chain order than $\mathrm{BA}$. (C) A distinguishing feature for the chain ordering is the orientation of Phe17, which would clash in a completely symmetric structure but in the asymmetric MexR-wt structures (ribbon) showing alternate orientations of Phe17 in all MexR dimers and in the MexR-ArmR complex, where Phe17 either points away from ('-" chain) or towards ("+' chain) H6/H6'. (D) The variation in all side-chain orientations (chil) was compared to the variation within the $-/+$ grouping using an F-test for equal variance. Shown are the resulting $-\log (\mathrm{P}$-values) for an equal variance test (F-test) of the $-/+$ grouping for the side-chain orientation (chi1). In this test Phe17 clearly has a significantly $(* * *$ level) smaller in-group compared to all group variance, again highlighting the clustering in side-chain orientation observed above. Leu13 also stands out at the (** level). High values indicate a possible clustering in side-chain orientation as a result of the grouping of chains of similar asymmetry. Notably, Phe17 stands out by having a mean chil()$=-176^{\circ}\left(\operatorname{std} \_\operatorname{dev}=7.1^{\circ}\right), \operatorname{chi} 1(+)=-75^{\circ}\left(3.1^{\circ}\right)$, while chil $($ all $)=-125^{\circ}\left(51^{\circ}\right)$, and Leu13 also appears with distinct Chils relative to the mean: Leu13, $-89^{\circ}\left(15^{\circ}\right),-165^{\circ}\left(12^{\circ}\right),-127^{\circ}\left(40^{\circ}\right)$. 
Figure S4
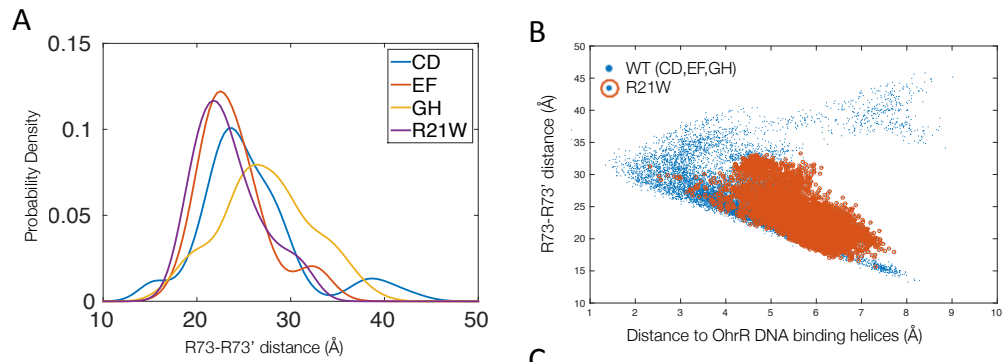

C

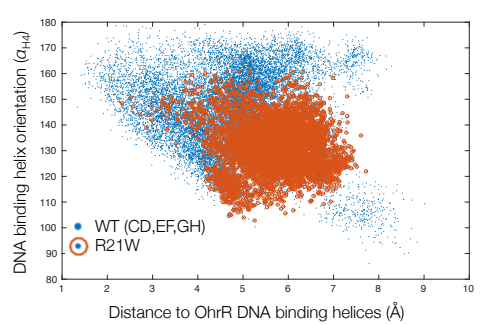

Figure S4, related to Figure 1. Distance Measure Relationships on MD trajectories. (A) Distribution of the inter-DNA domain spacing measured by the $\mathrm{C}_{\alpha}-\mathrm{C}_{\alpha}$ distance between R73-R73' in the DNA binding helices $\mathrm{H} 4 / \mathrm{H} 4$ ' over all trajectories starting from $\mathrm{CD}, \mathrm{EF}, \mathrm{GH}$, and R21W. (B) Scatter plot of the distance to OhrR DNA binding helices H4/H4' vs. R73R73' distance over all trajectories with points from R21W circled in orange. (C) Scatter plot of the distance to OhrR DNA binding helices $\mathrm{H} 4 / \mathrm{H} 4$ ' vs. DNA binding helix orientation $\left(\alpha_{\mathrm{H} 4}\right)$, see Figure 1 for definition of $\alpha_{\mathrm{H} 4}$, over all trajectories with points from R21W circled in orange. Previous studies have used R73-R73' distance to measure to assess the ability to bind DNA. The problem with a distance measure is that it does not take the internal or the relative the orientation of the helices into account. It is true that it is a necessary for inter-DNA domain spacing to be large enough to accommodate DNA, as shown in (B) the R73-R73' distance is $>25 \AA$ when the distance to the DNA binding helices is $<3.5 \AA$. But it is not sufficient since there are many R73-R73' distances $>25 \AA$ that also have a large distance to the DNA binding helices. In particular almost all trajectories starting from R21W with R73-R73' distance $>25 \AA$ have distances to the DNA binding helices $>4 \AA$. A similar trend is also observed for the DNA binding helix orientation $(\mathbf{C})$, the helix orientation has to be $>130$ degrees for the distance to DNA binding helices to be $<3.5 \AA$. But many helix orientations $>130$ degrees also have very large distances to the DNA binding helices, in particular, again, all trajectories starting from R21W have DNA binding helices distances $>4 \AA$ even if the helix orientation is $>130$ degrees. Thus, neither R73-R73' distance nor the DNA binding helix orientation alone are good measures to assess the ability to bind DNA. 


\section{Figure S5}

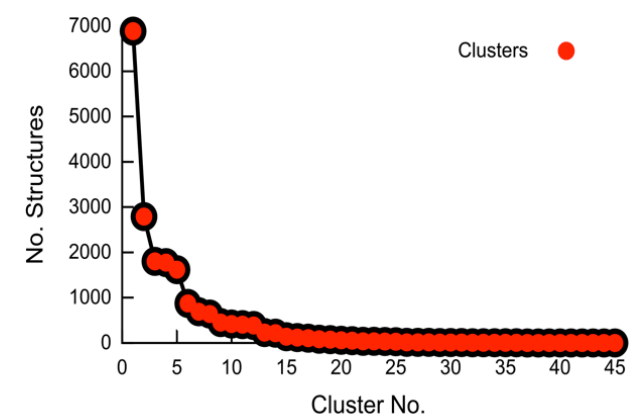

\begin{tabular}{|c|c|c|c|c|c|}
\hline Cluster & Structures & of total & Cluster & Structures & of total \\
\hline 1 & 6884 & $34 \%$ & 26 & 16 & $8.0 \cdot 10^{-2} \%$ \\
\hline 2 & 2788 & $14 \%$ & 27 & 14 & $7.0 \cdot 10^{-2} \%$ \\
\hline 3 & 1804 & $9 \%$ & 28 & 13 & $6.5 \cdot 10^{-2} \%$ \\
\hline 4 & 1773 & $9 \%$ & 29 & 10 & $5.0 \cdot 10^{-2} \%$ \\
\hline 5 & 1619 & $8 \%$ & 30 & 9 & $4.5 \cdot 10^{-2} \%$ \\
\hline 6 & 872 & $4 \%$ & 31 & 8 & $4.0 \cdot 10^{-2} \%$ \\
\hline 7 & 690 & $3 \%$ & 32 & 6 & $3.0 \cdot 10^{-2} \%$ \\
\hline 8 & 637 & $3 \%$ & 33 & 6 & $3.0 \cdot 10^{-2} \%$ \\
\hline 9 & 450 & $2 \%$ & 34 & 6 & $3.0 \cdot 10^{-2} \%$ \\
\hline 10 & 420 & $2 \%$ & 35 & 4 & $20 \cdot 10^{-2} \%$ \\
\hline 11 & 404 & $2 \%$ & 36 & 3 & $15 \cdot 10^{-2} \%$ \\
\hline 12 & 386 & $2 \%$ & 37 & 2 & $10 \cdot 10^{-2} \%$ \\
\hline 13 & 229 & $1 \%$ & 38 & 2 & $10 \cdot 10^{-2} \%$ \\
\hline 14 & 214 & $1 \%$ & 39 & 2 & $10 \cdot 10^{-2} \%$ \\
\hline 15 & 136 & $1 \%$ & 40 & 1 & $5.0 \cdot 10^{-3} \%$ \\
\hline 16 & 118 & $1 \%$ & 41 & 1 & $5.0 \cdot 10^{-3} \%$ \\
\hline 17 & 107 & $1 \%$ & 42 & 1 & $5.0 \cdot 10^{-3} \%$ \\
\hline 18 & 83 & $4.1 \cdot 10^{-1} \%$ & 43 & 1 & $5.0 \cdot 10^{-3} \%$ \\
\hline 19 & 75 & $3.7 \cdot 10^{-1} \%$ & 44 & 1 & $5.0 \cdot 10^{-3} \%$ \\
\hline 20 & 62 & $3.1 \cdot 10^{-1} \%$ & 45 & 1 & $5.0 \cdot 10^{-3} \%$ \\
\hline 21 & 49 & $24 \cdot 10^{-1} \%$ & & & \\
\hline 22 & 38 & $19 \cdot 10^{-1} \%$ & & & \\
\hline 23 & 37 & $18 \cdot 10^{-1} \%$ & & & \\
\hline 24 & 30 & $15 \cdot 10^{-1} \%$ & & & \\
\hline 25 & 28 & $14 \cdot 10^{-1} \%$ & TOTAL & 20040 & $100 \%$ \\
\hline
\end{tabular}

Figure S5, related to Figure 2. PC projections of RMSD clusters.

The number of conformations in the 45 clusters found with corresponding table shown. 
Figure S6

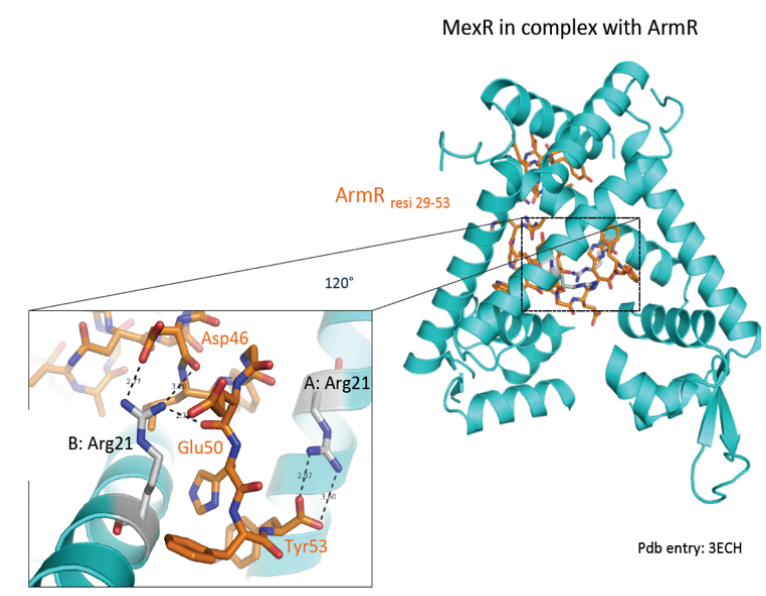

\begin{tabular}{|l|l|l|l|}
\hline ArmR & MexR & Distance, $\AA$ & Type \\
\hline Tyr53 (0) & A: Arg21 & 3.60 & Hydrogen bond \\
\hline Tyr53 (OXT) & A: Arg21 & 2.97 & Hydrogen bond \\
\hline Asp46 (002) & B: Arg21 & 2.82 & $\begin{array}{l}\text { Hydrogen bonding } \\
\text { H electrostatic interaction }\end{array}$ \\
\hline Asp46 (OD2) & B: Arg21 & 2.77 & Hydrogen bond \\
\hline Asp46 (0) & B: Arg21 & 3.20 & Hydrogen bond \\
\hline Glu50(0) & B: Arg21 & 2.74 &
\end{tabular}

Figure S6, related to Figure 3. MexR bound to ArmR peptide structure. Cartoon representation of MexR (cyan) bound to ArmR peptide (orange, shown as sticks) (PDB code - 3ECH). The table lists the H-bonds of MexR residue Arg21 from both subunits of the dimer contacting the ArmR peptide. 
Table S1, related to Figure 4. SAXS data collection, processing and derived structural parameters

\begin{tabular}{|c|c|c|c|}
\hline \multicolumn{2}{|c|}{ Data collection parameters } & \multicolumn{2}{|c|}{ MexR WT and R21W mutant } \\
\hline \multicolumn{2}{|c|}{ Instrumentation } & \multicolumn{2}{|l|}{ SAXSess, AntonPaar } \\
\hline \multicolumn{2}{|c|}{ Beam geometry } & \multicolumn{2}{|c|}{$\begin{array}{l}10 \mathrm{~mm} \text { slit, trapezoid approximation for GNOM: } \mathrm{AH}=0.23 \AA^{-1} \text {, } \\
\mathrm{LH}=0.13 \AA^{-1}\end{array}$} \\
\hline \multicolumn{2}{|c|}{$q$-range measured $\left(\AA^{-1}\right)$} & \multicolumn{2}{|c|}{$0.014-0.59(w t), 0.017-0.59(\mathrm{R} 21 \mathrm{~W})$} \\
\hline \multicolumn{2}{|c|}{ Exposure time } & \multicolumn{2}{|c|}{ A total of $60 \mathrm{~min}$ in four repetitions of $15 \mathrm{~min}$ each } \\
\hline \multicolumn{2}{|c|}{ Sample details } & MexR-wt* & MexR-R21W \\
\hline \multicolumn{2}{|c|}{ Residues in analyzed protein: } & $\begin{array}{l}\text { MexR }_{1-147}+\mathrm{N} \text {-terminal } \\
\text { GS residues }\end{array}$ & $\mathrm{MexR}_{5-139}+\mathrm{N}$-terminal GS residues \\
\hline \multirow{2}{*}{\multicolumn{2}{|c|}{$\begin{array}{l}\text { Protein concentration range } \\
\text { measured }\left(\mathrm{mg} \mathrm{mL}^{-1}\right) \\
\text { Partial specific volume from } \\
\text { sequence } \\
\left(v, \mathrm{~cm}^{3} \mathrm{~g}^{-1}\right)\end{array}$}} & 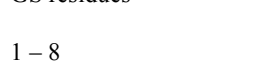 & $2-5$ \\
\hline & & 0.740 & 0.741 \\
\hline \multicolumn{2}{|c|}{$\begin{array}{l}\text { Contrast from sequence and } \\
\text { solvent constituents }\left(\Delta \rho, 10^{10}\right. \\
\left.\mathrm{cm}^{-2}\right)\end{array}$} & 2.746 & 2.734 \\
\hline \multicolumn{2}{|c|}{$\begin{array}{l}\text { Molecular weight from } \\
\text { sequence }(\mathrm{Da})\end{array}$} & 34,213 & 31,821 \\
\hline \multicolumn{2}{|c|}{$\begin{array}{l}R_{g}, d_{\max }(\AA) \text { for crystal } \\
\text { structure based models (dimer } \\
\text { chains) }\end{array}$} & $\begin{array}{l}22.8,80(\mathrm{CD}) \\
22.4,80(\mathrm{EF}) \\
22.6,80(\mathrm{GH})\end{array}$ & $21.6,70(\mathrm{AB})$ \\
\hline \multicolumn{4}{|c|}{ Structural parameters } \\
\hline \multirow{2}{*}{\multicolumn{2}{|c|}{$\begin{array}{l}\text { Experimental temperature } \\
\left({ }^{\circ} \mathrm{C}\right) \\
{ }^{\text {PProtein concentration } \mathrm{mg}} \\
\mathrm{mL}^{-1}\end{array}$}} & 30 & 30 \\
\hline & & 2.0 & 2.1 \\
\hline Guinier & $R_{g}(\AA)$ & $25.8 \pm 0.1$ & $23.7 \pm 0.4$ \\
\hline Analysis & $q R_{g}$-limit & 1.3 & 1.3 \\
\hline $\begin{array}{l}P(r) \\
\text { Analysis }\end{array}$ & $\begin{array}{l}R_{g}(\AA) \\
d_{\max }(\AA) \\
q \text {-range } \\
* M W \text { from } I(0)\end{array}$ & $\begin{array}{l}25.4 \pm 0.2 \\
80 \\
0.014-0.2 \\
39,432\end{array}$ & $\begin{array}{l}23.6 \pm 0.2 \\
75 \\
0.017-0.2 \\
34,977\end{array}$ \\
\hline
\end{tabular}

* MexR WT lacks aromatic Trp residue, thus may contribute for an error in concentration

determination using $\mathrm{UV}_{280}$. Additional Bradford assays were performed and the mean value used to calculate $M W$ values.

${ }^{\#}$ At $30^{\circ} \mathrm{C}$ samples showed evidence of aggregation above $2 \mathrm{mg} / \mathrm{mL}$ preventing meaningful structural analysis for the higher concentration data.

Errors quoted are propagated counting statistics only. 
Table S2, related to Figure 3. List of MexR residues that participate in the different contact network clusters depicted in Main Figure 3. MexR-WT and R21W clusters are listed separately. Chain A is (-) and chain B is (+), using the annotation described in Figure S3.

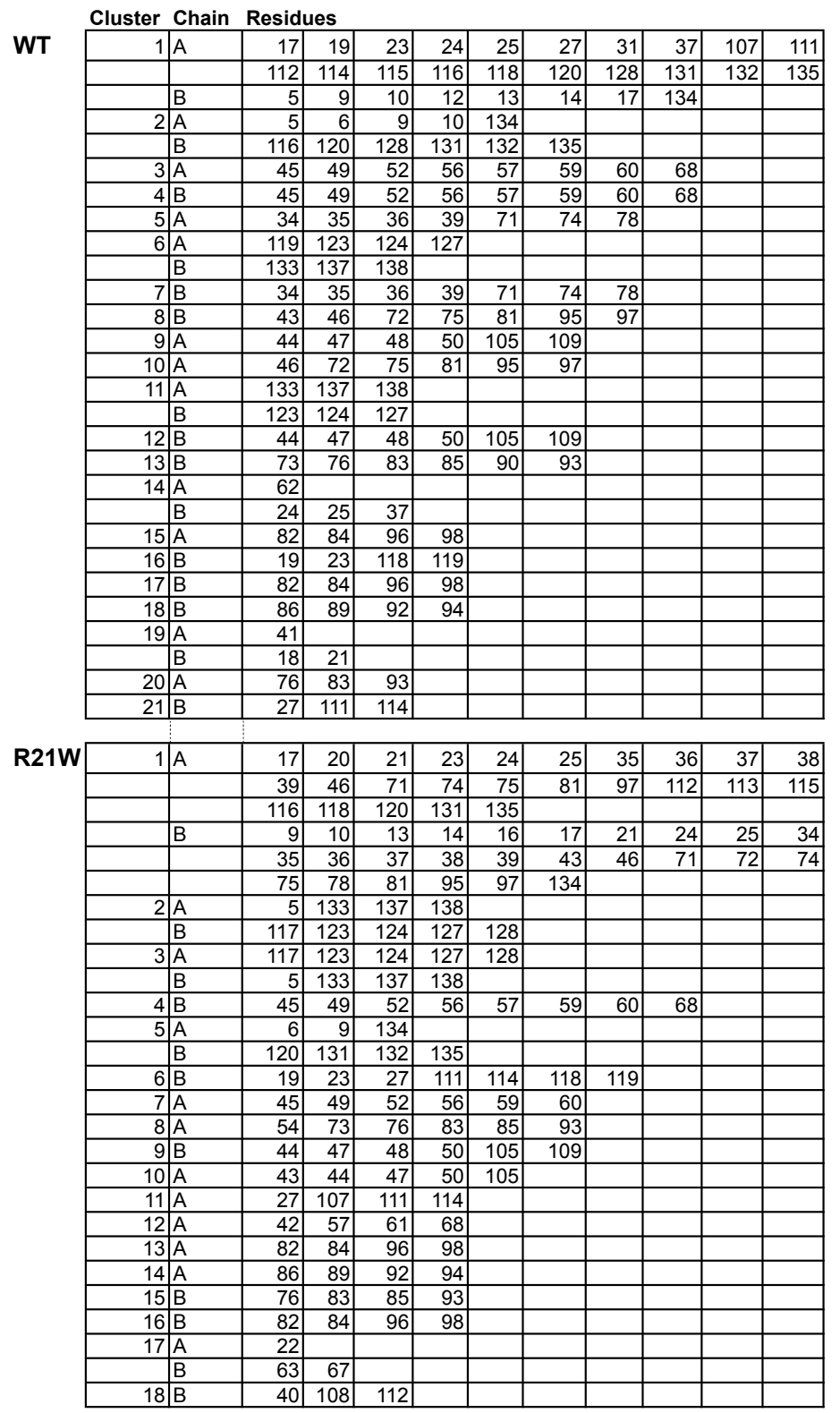





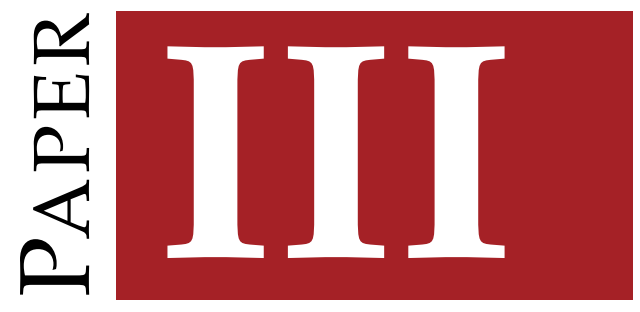

Arne Elofsson, Keehyoung Joo, Chen Keasar, Jooyoung Lee, Ali H. A. Maghrabi, Balachandran Manavalan, Liam J. McGuffin, David Ménendez Hurtado, Claudio Mirabello, Robert Pilstål, Tomer Sidi, Karolis Uziela, and Björn Wallner. "Methods for estimation of model accuracy in CASP12". eng. In: Proteins (Oct. 2017). ISSN: 1097-0134. DOI: 



\title{
Methods for estimation of model accuracy in CASP12
}

\author{
Arne Elofsson ${ }^{1}$ (D) | Keehyoung Joo ${ }^{2}$ | Chen Keasar ${ }^{3}$ | Jooyoung Lee ${ }^{4}$ (1) । \\ Ali H. A. Maghrabi5 | Balachandran Manavalan4 | Liam J. McGuffin ${ }^{5}$ (i) | \\ David Ménendez Hurtado ${ }^{1}$ | Claudio Mirabello ${ }^{6}$ | Robert Pilstål6 | Tomer Sidi ${ }^{3}$ | \\ Karolis Uziela ${ }^{1}$ | Björn Wallner6 (1)
}

\begin{abstract}
${ }^{1}$ Department of Biochemistry and Biophysics and Science for Life Laboratory, Stockholm University, Box 1031, Solna 171 21, Sweden ${ }^{2}$ Center for In Silico Protein Science and Center for Advanced Computation, Korea Institute for Advanced Study, Seoul 130-722, Korea ${ }^{3}$ Department of Computer Science, Ben Gurion University of the Negev, Israel

${ }^{4}$ Center for In Silico Protein Science and School of Computational Sciences, Korea Institute for Advanced Study, Seoul 130-722, Korea ${ }^{5}$ School of Biological Sciences, University of Reading, Whiteknights, Reading RG6 6AS, United Kingdom

${ }^{6}$ Department of Physics, Chemistry, and Biology, Bioinformatics Division, Linköping University, Linköping 581 83, Sweden
\end{abstract}

Correspondence

Arne Elofsson, Department of Biochemistry and Biophysics and Science for Life Laboratory, Stockholm University, Box 1031, Solna 171 21, Sweden. Email: arne@bioinfo.se

\section{Funding information}

This work was supported by grants from the Swedish Research Council (VR-NT 2012-5046 to AE and 2012-5270 to B.W. and Swedish e-Science Research Center (B. W.). Computational resources were provided by the Swedish National Infrastructure for Computing (SNIC) at NSC.

Manavalan, Joo and Lee were supported by the National Research Foundation of Korea (NRF) grant funded by the Korea government (MEST) (No. 2008-0061987). We are grateful for the Saudi Arabian Government Studentship to A.H.A Maghrabi. Chen Keasar and Tomer Sidi are grateful for support by grant no. 2009432 from the United States-Israel Binational Science Foundation (BSF) and grant no. 1122/14 from the Israel Science Foundation (ISF).

\section{1 | INTRODUCTION}

Estimates of model accuracy (EMA) have been a part of protein structure prediction since its infancy. EMA methods are built into virtually all protein $3 \mathrm{D}$ modeling methods as the energy functions that they optimize. Yet, these energy functions provide relative accuracy

All authors contributed equally to this study and the list is sorted alphabetically.

\begin{abstract}
Methods to reliably estimate the quality of 3D models of proteins are essential drivers for the wide adoption and serious acceptance of protein structure predictions by life scientists. In this article, the most successful groups in CASP12 describe their latest methods for estimates of model accuracy (EMA). We show that pure single model accuracy estimation methods have shown clear progress since CASP11; the 3 top methods (MESHI, ProQ3, SVMQA) all perform better than the top method of CASP11 (ProQ2). Although the pure single model accuracy estimation methods outperform quasi-single (ModFOLD6 variations) and consensus methods (Pcons, ModFOLDclust2, Pcomb-domain, and Wallner) in model selection, they are still not as good as those methods in absolute model quality estimation and predictions of local quality. Finally, we show that when using contact-based model quality measures (CAD, IDDT) the single model quality methods perform relatively better.
\end{abstract}

KEYWORDS

CASP, consensus predictions, estimates of model accuracy, machine learning, protein structure prediction, quality assessment

estimates, with only moderate power in properly ranking models. Further, when one tries to use models from different methods, their associated energies are not directly comparable. Thus, accurate posterior quality estimation methods are essential for protein structure prediction tools to fulfill their potential as useful techniques for biologists.

Motivated by the intriguing experiment of Novotny et al. ${ }^{1}$ early model accuracy assessment methods focused on distinguishing wrong models (or decoys) from the native structure. ${ }^{2,3}$ Historically, knowledge-based energy functions were developed to solve this 


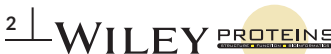

TABLE 1 Summary of the best performing QA methods in CASP12 and comments about their strength and weaknesses

\begin{tabular}{|c|c|c|c|}
\hline Methods & Type & Comment about global performance & Comment about local performance \\
\hline $\mathrm{MESH}^{32}$ & Single & Top model selection & $\mathrm{N} / \mathrm{A}$ \\
\hline MESHI_con ${ }^{32}$ & Single ${ }^{a}$ & Top model selection & $\mathrm{N} / \mathrm{A}$ \\
\hline $\operatorname{ProQ}^{21}$ & Single & Good model selection & Acceptable local scores \\
\hline $\operatorname{ProQ}^{17}$ & Single & Top model selection & Good local scores \\
\hline$S_{S M Q A}{ }^{34}$ & Single & Top model selection & N/A \\
\hline ModFOLD6 ${ }^{16}$ & Quasi-single & Balanced performance & Good assignment of local scores \\
\hline ModFOLD6_rank ${ }^{16}$ & Quasi-single & Acceptable model selection & Identical to ModFOLD6 \\
\hline ModFOLD6_cor ${ }^{16}$ & Quasi-single & Best absolute but suboptimal model selection & Identical to ModFOLD6 \\
\hline qSVMQA $^{34}$ & Quasi-single & Assignment of the absolute score is not accurate. & N/A \\
\hline ModFOLDclust $2^{46}$ & Clustering & $\begin{array}{l}\text { Good assignment of absolute global scores but suboptimal } \\
\text { model selection }\end{array}$ & Top assignment of local scores \\
\hline Pcons ${ }^{11}$ & Clustering & Good assignment of absolute global scores & Top assignment of local scores \\
\hline Pcomb-domain ${ }^{13}$ & Combined & $\begin{array}{l}\text { Good assignment of absolute global scores, requires good } \\
\text { domain prediction }\end{array}$ & Top assignment of local scores \\
\hline Wallner & Combined & Good assignment of absolute global scores & Top assignment of local scores \\
\hline
\end{tabular}

Methods basically identical have been merged.

a MESHI_con is not pure single methods but requires multiple models to average the predictions.

problem and they were used in threading methods, as well as to guide protein folding and fragment assembly. Notably, the methods by Sippl, which used knowledge-based energy function for threading, were quite successful in CASP1-3., ${ }^{4,5}$ However, in later CASP experiments, pure threading methods were not able to compete with methods that also made use of use evolutionary information from the rapidly growing sequence databases.

None of the energy functions that were developed to distinguish native and nonnative protein models showed any major success in the quality assessment (QA) category in CASP. Instead, methods that aim to predict the quality of a model starting with ProQ, ${ }^{6}$ have been more successful. One of the notable features separating these methods from the earlier knowledge-based energy terms were the use of compatibility with predicted structural features, such as secondary structure. These methods are nowadays referred to as single model QA methods to distinguish them from methods that use clustering (or a consensus) of many models. Since the introduction of ProQ other methods based on the same idea have been introduced, including QMEAN ${ }^{7}$ that has performed comparably with ProQ in earlier CASPs. Initially the single model methods have not been as successful as those that take into account structural similarity of models, that is, consensus-based methods. ${ }^{8}$ Since CASP11, however, they perform on par or even better than the consensus methods in some of the tasks. ${ }^{8}$

The first successful attempt of model quality estimation, in the context of CASP, was when the first meta-predictor, CAFASP-CONSENSUS, was introduced in CASP4. ${ }^{9}$ CAFASP-CONSENSUS combined the results from several servers and provided better models than any of the individual servers. However, in CASP4 the model quality estimates were carried out manually. From this exercise, it was discovered that using simple rules for combining the predictions from severa servers could outperform all individual servers. This algorithm simply chose the most frequent fold predicted by all servers, that is, it chose the consensus fold. ${ }^{9,10}$

Soon after CASP4, the first automatic consensus method, Pcons, was introduced. ${ }^{11}$ This was later followed by a simpler (and more robust) method, 3D-Jury. ${ }^{12}$ Later versions of Pcons are very similar to 3D-Jury, ${ }^{13}$ the only difference being in the details of the superposition method. In CASP5, it was clear that these methods could be used to outperform all individual servers if the results were combined. In CASP7, model accuracy estimation became a new category in and of itself for the first time. ${ }^{14}$

Quasi-single model methods, such as the latest ModFOLD servers $^{15,16}$ compare a model with models generated by a local predictionpipeline using the consensus approach. These methods, as well as Pcomb $^{13}$ that uses the Pcons consensus approach, combine the consensus score with one or several pure single model approaches. The performance of the best quasi-single approaches often match the performance of the consensus methods, but with the ability to evaluate a single model at a time given that a set of external predictions exist.

In this article, we will briefly describe each of the EMA methods used by our groups in CASP12. Additionally, we will compare the relative performance of the methods, discuss their relative strengths and weakness and we will share our insights on what we learned from the experiment this time round.

\section{2 | METHODS}

A summary of all methods discussed in this article is presented in Table 1. Below, each group briefly presents their methods. 
TABLE 2 Description of the evolution of ProQ methods used in CASP and their relative performance

\begin{tabular}{lll} 
Method & Major novelty & Correlation global/local \\
\hline ProQ $^{6}$ & $\begin{array}{l}\text { First method trained to predict "quality" of a model. Using a combination of structural } \\
\text { descriptions and agreement with predicted secondary structure. }\end{array}$ & $0.71^{\mathrm{a}} /-$ \\
\hline ProQres $^{20}$ & Predicting local qualities-global quality is sum of local quality. & $-/ 0.56^{\mathrm{b}}$ \\
\hline ProQ2 $^{21}$ & Global agreement with predicted RSA and SS plus profile weighting. Uses a linear kernel SVM. & $0.80 / 0.71^{\mathrm{b}}$ \\
& & $0.84 / 0.72^{\mathrm{C}}$ \\
\hline ProQ3 $^{17}$ & Added rosetta energies to the inputs. & $0.87 / 0.74^{\mathrm{C}}$ \\
\hline ProQ3D $^{18}$ & Linear kernel SVM is replaced by a 2-layer perceptron. & $0.91 / 0.77^{\mathrm{C}}$
\end{tabular}

${ }^{a}$ From original ProQ publication. ${ }^{6}$

${ }^{\mathrm{b}}$ From ProQ2 publication. ${ }^{21}$

${ }^{\mathrm{C}}$ On CASP11 dataset trained on CASP9 and CASP10.

\section{1 | Elofsson group}

We participated with several accuracy estimation methods in CASP12. Here, we will highlight the 2 methods that performed best; the single model accuracy estimation tool ProQ $3^{17}$ and our consensus-based method Pcons. ${ }^{11}$ Our other methods included an early version of ProQ3D ${ }^{18}$ the deep learning version of ProQ3. ProQ3_diso is a version of ProQ3 where disordered residues are ignored and RSA_SS is a simple QA method that only utilizes predicted secondary structure and surface area. For details see the CASP 12 abstracts at http://predictioncenter.org/casp12/doc/ CASP12_Abstracts.pdf.

ProQ $^{17}$ is the latest version of our single model accuracy estimation methods. ${ }^{6,19-21}$ In Table 2 we describe the most important developments in the history of ProQ. In addition to using the same descriptions of a model as ProQ2 ${ }^{21}$ it also uses Rosetta energy functions. All input features are combined together to train a linear SVM. The training dataset is a subset of CASP9 with 30 models per target. We also tested a few developmental methods of ProQ in CASP12, but none of these performed significantly better than ProQ3 and they are therefore not discussed here. However, it can be noted that we have recently developed an improved version of ProQ3, ProQ3D that uses a deep-learning approach but identical inputs as ProQ3 ${ }^{18}$ The final version was not ready for CASP12 and the preliminary version used did not perform better than ProQ3. ProQ3 is available both as source code from https://bitbucket.org/ElofssonLab/proq3, and as a web-server at http://proq3.bioinfo.se/.

Pcons ${ }^{11}$ is used with default setting. This means that the score is calculated by performing a structural superposition using the algorithm described by Levitt and Gerstein ${ }^{22}$ of a model against all other models. To avoid bias, comparisons between models from the same method are ignored. After superposition, the "S-score" is calculated for each residue in the model. ${ }^{23}$ The average S-score for all residues and pairs of models is then used to calculate the final Pcons score. For local predictions, the average $\mathrm{S}$-score is converted to a distance as described before. ${ }^{13}$ Pcons is freely available from https://github.com/bjornwallner/Pcons/. It should be noted that a number of heuristic optimizations have been implemented in Pcons to enable the pairwise comparison of hundreds of proteins in a short time. ${ }^{24}$

\section{2 | Keasar group}

We participated in CASP12 with 2 EMA methods, MESHI-score (implemented by the MESHI_server group) and MESHI-score-con (MESHI_con_server), the latter being a slight variation on the former. Below we first present the general scheme, which is used by both methods, and then conclude with the variations tried in MESHI-scorecon.

Although preliminary versions of MESHI-score were used in CASP10 and CASP11, it has reached stability only after CASP $11 .{ }^{25}$ The software architecture, however, is modular, extendable by design, and under continuous development. Thus, the version that took part in CASP12 was more advanced than what was presented earlier. ${ }^{25}$

The MESHI-score pipeline (Figure 1) starts with a regularization step that includes side-chain repacking by SCWRL ${ }^{26,27}$ and restrained energy minimization (Figure 1, II). This step sharpens the quality signal of structural features by reducing noise, which is due to peculiarities of decoy generating methods. Features are extracted from the regularized structures (Figure 1, III) and fed to an ensemble of 1000 independently trained predictors (Figure 1, IV). Each predictor outputs $\left(s_{i}, w_{i}\right)$, a pair of an EMA score and weight (Figure $1 \mathrm{VI})$. The weighted median of this set of pairs is the final MESHI-score (Figure 1VII). In addition, we also calculate the weighted interdecile range and entropy of the pairs set:

$$
\operatorname{entropy}(S)=\sum_{j=1}^{100} P_{j} \log _{2}\left(P_{j}\right)
$$

where $S$ is the set of $1000\left(s_{i}, w_{i}\right)$ pairs and

$$
P_{j}=\sum_{j=1}^{1000} \frac{w_{i}}{Q} l_{j}\left(s_{i}\right), Q=\sum_{i=1}^{1000} w_{i}
$$

and

$$
l_{j}(s)=1 \text { iff }(j-1) \leq s<0.01 j \text { otherwise } l_{j}(s)=0
$$

The larger these numbers are the less reliable is the score, as they suggest disagreement between the predictors.

The feature set that was used in CASP12 included 82 features (for details see https://www.cs.bgu.ac.il/ frankel/TechnicalReports/2015/ 15-06.pdf)

These features may be clustered into nine broad categories: 


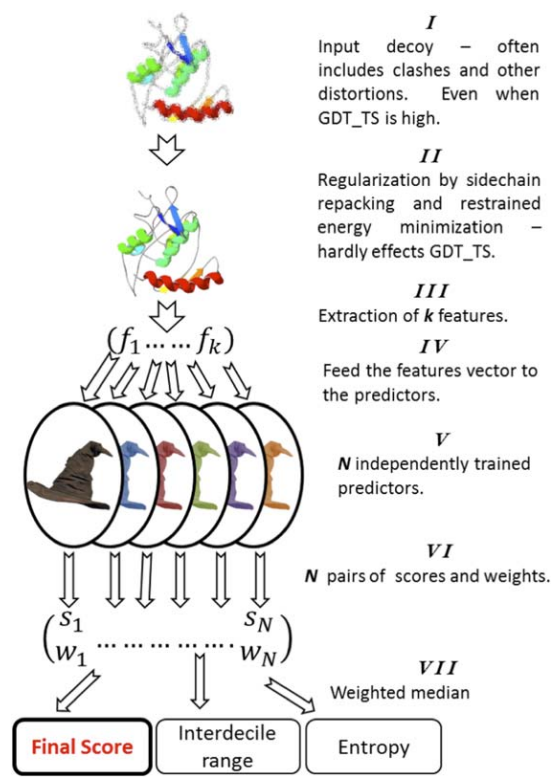

FIGURE 1 Flowchart outlining the principal stages of the ModFOLD6 server prediction pipeline. The initial input data are the target sequence and a single 3D model. The output data are the local/per-residue scores from the ModFOLD6 NN and the global score variants-ModFOLD6, ModFOLD6_rank, and ModFOLD6_cor. The ModFOLD6 pipeline is dependent on the following methods PSIPRED ${ }^{31}$ DISOPRED, ${ }^{36}$ and MetaPSICOV ${ }^{37}$ [Color figure can be viewed at wileyonlinelibrary.com]

1. Pairwise energy terms, which represent interactions between atoms, adopted from the literature. ${ }^{28-30}$

2. Compatibility of the decoy secondary structure and solvent accessibility with their PSIPRED ${ }^{31}$ prediction.

3. Standard bonded energy terms (eg, a quadratic bond term)

4. Torsion angle terms (compatibility with Ramachandran plot and rotamer preferences ${ }^{32}$.

5. Hydrogen bond terms. ${ }^{33}$

6. Solvation and atom environment terms that quantify the cooperativity between hydrogen bond formation and atom burial.

7. Radius of gyration and contact terms that quantify the compatibility of decoys with the expected, length dependent, ratios between the radii of gyration and numbers of contacts in different subsets of protein atoms (eg, polar and hydrophobic).

8. Meta-features that quantify the frustration within decoys (native structures tend to be minimally frustrated) by considering the distribution of the pairwise and torsion energies within the decoys.

9. Combinations of the above features, which were developed in previous studies. ${ }^{25}$
The predictors (Figure $1 \mathrm{~V}$ ) are nonlinear functions that get feature vectors as an input and output a pair of numbers: an EMA score, and a weight that represents the reliability of the score. The parameters of the predictor functions, as well as the subset of features that they use, are learned by stochastic optimization. Each predictor is trained to minimize a different objective function and thus tends to be more sensitive in a specific GDT TS subrange. Scores within the predictor's sensitivity region are considered more reliable and thus, have a higher weight. A more detailed description of the predictor's training may be found in Mirzaei et al. ${ }^{25}$

MESHI-score-con is a variant on the MESHI-score theme, which aims to improve the consistency of MESHI-score by a post-processing step that takes into account the similarities between decoys. Ideally, after regularization (Figure 1, II) very similar decoys should produce similar feature vectors, and thus have similar MESHI-scores. Yet, careful examination of MESHI-score results indicates that this is not always the case, and often very similar decoys have quite different scores. MESHIscore-con aims to alleviate this problem by improving the agreement between the scores of very similar decoys. To this end, we associate the MESHI-score of each decoy with a weight, which is inversely proportional to the entropy of the score-weight pairs (Figure 1VII). We also associate each decoy with a neighbors-set that includes very similar (GDT TS > 95) neighbors as well as the decoy itself. MESHI-score-con is a weighted average of the decoy's MESHI-score and the average score of its neighbor-set. Thus, a low weight decoy (presumably a less reliable one) with higher weight neighbors is strongly biased toward the average score of its neighbors. Yet the score of a decoy without neighbors is unaffected regardless of its weight. Thus, unlike consensus methods MESHI-score-con may pick an exceptionally good decoy.

\section{3 | Lee group}

We participated in CASP12 with 2 methods, namely SVMQA and quasi-SVMQA (qSVMQA). qSVMQA augments TM-score between GOAL_TS1 and the server model with an appropriate value of weight $w$ to the SVMQA score:

$$
\text { qSVMQA }=\text { SVMQA }+w^{*}(T M-\text { score between }
$$$$
\text { GOAL_TS1 and the server model). }
$$

The value of $w$ was set separately for stage1 models (0.84) and for stage2 models (0.15). We determined the optimal value of $w$ using CASP11 single-domain targets. Below, we briefly describe SVMQA and highlight its results in the model selection of stage 2 targets in CASP12

SVMQA is a support vector machine-based protein single-model global QA method. SVMQA predicts the global QA score as the average of the predicted TM-score and GDT_TS score by combining 2 separate predictors, SVMQA_GDT and SVMQA_TM. For SVMQA we used 19 features (8 potential energy-based terms and 11 consistency-based terms between the predicted and actual values of the model) for predicting the QA score (TM-score or GDT_TS score). Among these 19 features, 3 features (orientation dependent energy, GOAP angular energy and solvent accessibility consistency score) were not used in earlier versions, while the other 16 have been used in existing methods. The description of each feature along with the selection of the final set 


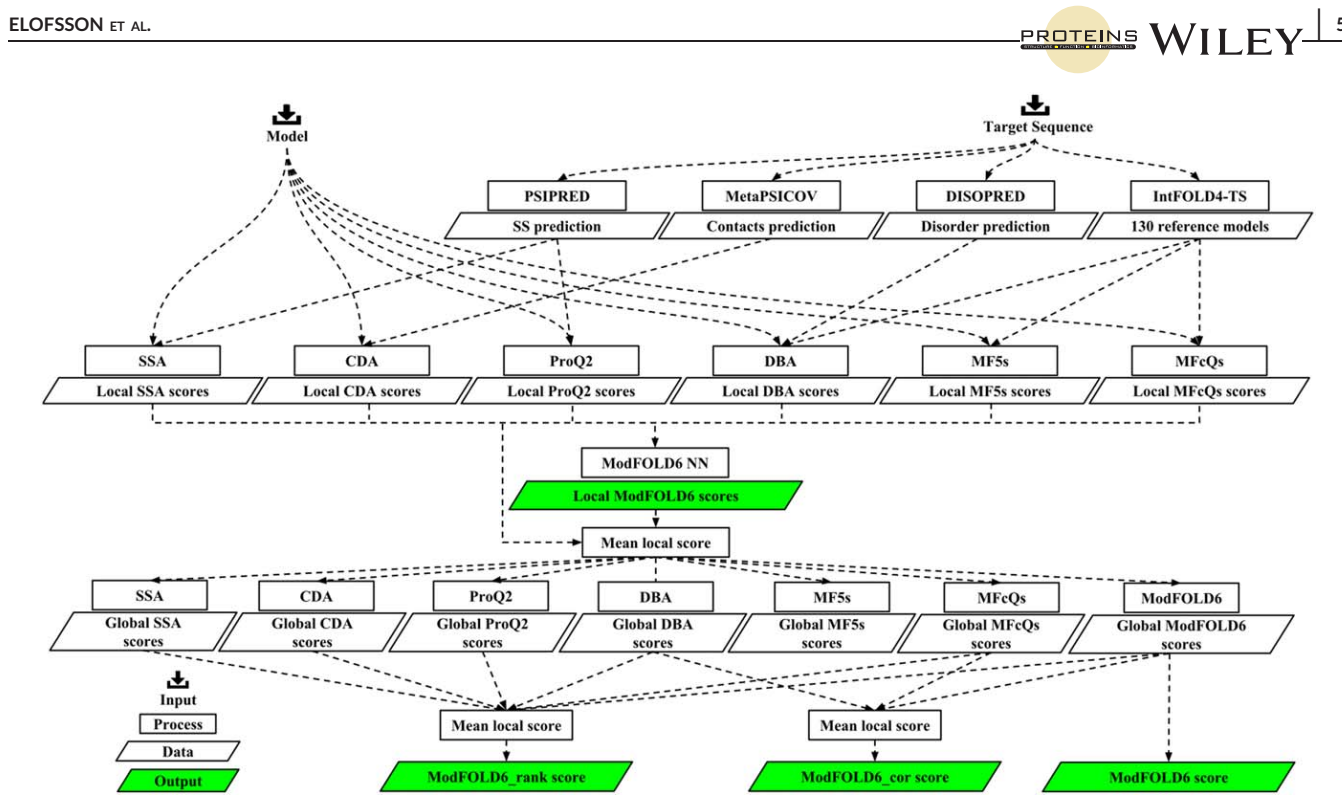

FIGURE 2 The MESHI-score pipeline starts with a regularization step that includes side-chain repacking by SCWRL ${ }^{26,27}$ and restrained energy minimization. Features are extracted from the regularized structures and fed to an ensemble of independently trained predictors. Each predictor outputs a pair of values: an EMA score and weight, and the weighted median of this set of pairs is the final MESHI-score [Color figure can be viewed at wileyonlinelibrary.com]

of SVM parameters and the final set of features for these 2 predictors have been published recently. ${ }^{34}$ In short, SVMQA_TM uses all of the 19 features to predict TM-score of a given model, whereas SVMQA_GDT uses only 15 features to predict the GDT_TS score.

In CASP11, we used our old QA method, RFMQA. ${ }^{35}$ The result of RFMQA on CASP11 targets was quite successful but not as good as that of SVMQA on CASP12 targets. Prior to CASP12, we benchmarked the performance of SVMQA on CASP11 targets and compared it to that of RFMQA. We found that SVMQA significantly outperformed RFMQA in terms of both ranking models and selecting a more nativelike model. The major updates of SVMQA over RFMQA is as follow: (i) The choice of machine learning method was different, an SVM was used in SVMQA while a random forest was used in RFMQA; (ii) we used CASP8-9 domain targets as the training dataset for RFMQA, while CASP8-10 domain targets were used in SVMQA; (iii) 19 input features were used in SVMQA, whereas, only 9 of these features were used in RFMQA; (iv) The objective function to train for RFMQA was $T M_{\text {loss }}$ (difference between the TM-score of the selected model and the best TM-score), while that for SVMQA was the correlation coefficient between the actual ranking and the predicted ranking; and $(\mathrm{v})$ SVMQA used 2 separate predictors for TM-score and GDT TS score, while RFMQA used only a predictor for TM-score.

\section{4 | McGuffin group}

We participated in CASP12 with 3 new quasi-single model method variants, ModFOLD6, ModFOLD6_cor, and ModFOLD6_rank (Figure 2), and one older clustering method, ModFOLDclust2.

\subsection{1 | ModFOLD6}

The ModFOLD6 server ${ }^{16}$ is the latest version of our freely available public resource for the accuracy estimation of $3 \mathrm{D}$ models of proteins. ${ }^{15,38,39}$ The ModFOLD6 server combines a pure-single and quasisingle model strategy for improving accuracy of local and global model accuracy estimates. Our initial motivation in the development of ModFOLD6 was to increase the accuracy of local/per-residue assessments for single models. ${ }^{16}$

For the local/per-residue error estimates, each model was considered individually using 2 new pure-single model methods, the Contact Distance Agreement and the Secondary Structure Agreement scores, ${ }^{16}$ as well as the best pure single method in earlier CASPs, ProQ2.21,40 Additionally, 3 alternative quasi-single model methods were used to score models including: the newly developed Disorder B-factor Agreement, the ModFOLD5_single and the ModFOLDclustQ_single scores ${ }^{16}$-each of which made use of a set of 130 reference 3D models that were generated using the latest version of the IntFOLD-TS ${ }^{41,42}$ pipeline from the IntFOLD server. ${ }^{43,44}$ The component per-residue scores from each of the 6 alternative scoring methods, mentioned above, were combined into a single score for each residue using an Artificial Neural Network, which was trained to learn the local Sscore $^{23}$ as the target function ${ }^{16}$ (ie, the same target function as ProQ2, described below and in Table 2 was used, but with $\mathrm{d}_{0}$ set to 3.9).

For global scoring, in the ModFOLD6 variant we simply took the mean local score for each model (that is, the sum of the per-residues scores divided by the target sequence length). However in our internal benchmarks, using $\mathrm{CASP}_{1} 1^{8}$ and $\mathrm{CAMEO}^{45}$ data prior to CASP12, we 
realized that simply taking the mean per-residue score from ModFOLD6 alone was not optimal and performance differed depending on the intended use case, that is, selecting the best models or accurately reproducing the model-target similarity scores. Therefore we also exhaustively explored all linear combinations of each of the alternative global scores, in order to find the optimal mean score (OMS) for each major use case. ${ }^{16}$

\subsection{2 | ModFOLD6_cor}

The aim of developing the ModFOLD_cor global score variant was to optimize the correlations of predicted and observed global scores. In other words, the predicted global accuracy estimation scores produced by the method should have close to linear correlations with the observed global accuracy estimation scores. The OMS for the ModFOLD6_cor global score was found as:

(ModFOLDclustQ_single_global + DBA_global + ModFOLD6_global) $/ 3$ where the _global suffix indicates that the mean local score was taken for the scoring method indicated above.

\subsubsection{ModFOLD6_rank}

The aim of developing the ModFOLD6_rank global score variant was to optimize for the selection of the best models namely the top ranked models (top 1) should be closer to the highest accuracy, regardless of the relationship between the absolute values of predicted and observed scores. The OMS for the ModFOLD6_rank global score was found as:

(ModFOLDclustQ_single_global + ProQ2_global + CDA_global + DBA_global + SSA_global + ModFOLD6_global)/6.

Note that the local scores submitted for each of the $3 \mathrm{Mod}$ FOLD6 variants were identical and it was only the global scores (and therefore the ranking of models), which differed between the 3 ModFOLD6 variants. All 3 of the ModFOLD6 variants are freely available at: http://www.reading.ac.uk/bioinf/ModFOLD/ModFOLD6_form. html

\subsection{4 | ModFOLDclust2}

The ModFOLDclust2 method ${ }^{46}$ is a leading automatic clustering-based approach for both local and global 3D model accuracy estimation assessment. ${ }^{47,48}$ The ModFOLDclust2 server tested during CASP12 was identical to that tested during the CASP9, CASP10, and CASP11 experiments. The local and global scores have been previously described $^{46}$ and are unchanged since CASP9. Thus, the ModFOLD clust2 method serves as a useful gold standard/benchmark against which progress may be measured in the development of single mode methods. ModFOLDclust2 can be run as an option via the older ModFOLD3 server (http://www.reading.ac.uk/bioinf/ModFOLD/ModFOLD_form_3_0.html). The ModFOLDclust2 software is also available to download as a standalone program (http://www.reading.ac.uk/bioinf/downloads/).

\section{5 | Wallner group}

We participated with 3 EMA methods; ProQ2, ${ }^{21}$ Pcomb-domain, and Wallner.

ProQ2 is a single model accuracy estimation program based on a linear kernel SVM trained on a set of structural descriptors of a model. ProQ2 is trained to predict the local S-score ${ }^{23}$ :

$$
S_{i}\left(d_{i}\right)=\frac{1}{\left(1+\left(\frac{d_{i}}{d_{0}}\right)^{2}\right)}
$$

where $d_{i}$ is the local distance deviation for residue $i$ in the optimal superposition that maximize sum of $\mathrm{S}$ over the whole protein, and $\mathrm{d}_{0}$ is a distance threshold put to 3.0 here. The global score is the sum of local $S_{i}$ divided by the target length yielding a score in the range $[0,1]$. Local $\mathrm{S}$-scores, $\mathrm{S}_{\mathrm{i}}$, were converted to local distance deviation using the formula:

$$
d_{i}\left(S_{i}\right)=d_{0} * \sqrt{\left(\frac{1}{s_{i}}-1\right)}
$$

ProQ2 has participated in CASP since CASP10. Before CASP11 we implemented ProQ2 as a scoring function in Rosetta, ${ }^{40}$ enabling scoring and integration in any Rosetta protocol. ProQ2 was top-ranked in both CASP10 and CASP11. This inspired the development of novel methods including SVMQA, MESHI-score, and ProQ3. ProQ2 is also included in several hybrid methods used here. Therefore it could be claimed that ProQ is laying the foundation for the improvement in model QA apparent in CASP12. ProQ2 is also included in the topranked structure prediction methods BAKERROSETTA-SERVER ${ }^{49}$ and the IntFOLD4 server for TS prediction. ${ }^{50}$

Wallner method in this CASP is what was called Pcomb in earlier CASPs. ${ }^{13,51}$ It combines ProQ2 and Pcons using the following linear combination for global prediction ${ }^{20}$ :

Pcomb $=0.2^{*}$ ProQ2 $+0.8^{*}$ Pcons

For local prediction the same formula was used to calculate weighted local S-scores, which then were converted to distances using the $d_{i}\left(S_{i}\right)$ formula, described above.

Pcomb-domain method is a new domain-based version of Pcomb. Traditionally, consensus methods, including Pcons ${ }^{11}$ (https://github. com/bjornwallner/pcons), have always used rigid-body superposition for the full-length models, thereby selecting models that overall have the highest consensus, ignoring the fact that smaller domains from other models could have higher consensus over that region. To try to overcome this problem we developed a domain-based version of Pcons, which uses an initial domain definition. The domain-based Pcons scores were combined with local predicted scores from ProQ2. Two different methods were used to predict the domain boundaries of the target sequences, the first used the domain definitions from the Robetta server and the second was based on spectral analysis of the top ranking server models according to the regular Pcomb method. The results from these 2 methods were manually evaluated to decide the final domain boundaries. In addition, the Pcons and ProQ2 scores were weighted in a slightly different way compared the regular Pcomb 
method; following a parameter optimization based on targets released in the last 2 editions of CASP the relative weight for ProQ2 was increased to 0.3 resulting in this formula:

Pcomb - domain $=0.3^{*}$ ProQ2 - domain $+0.7^{*}$ Pcons - domain

Furthermore, $d_{0}$ was increased from 3.0 to $5.0 \AA$ as it showed improved model selection on CASP11, results not shown. Increasing $d_{0}$ shifts the sensitivity to lower quality (higher RMSD) residues, which is an advantage in CASP. As for both ProQ2 and Pcons all predictions are performed in the $S$-score space, global scores are sum of local scores, and the local $S$ scores are transformed to distances in the final step, using the $d_{i}\left(S_{\mathrm{i}}\right)$ formula above.

\section{3 | RESULTS}

A detailed analysis of CASP12 EMA methods is provided in the accompanying EMA assessment paper. ${ }^{52}$ In this section, we refer to the results provided in this article pertaining to our methods and also perform an additional analysis based on the correlation between different scores for different types of methods.

\section{1 | Global accuracy estimations in CASP12}

Here, we describe our analysis of our global accuracy estimation methods described in Table 1. As shown in the EMA assessment paper ${ }^{52} 3$ single model accuracy estimation methods (ProQ3, SVMQA, and MESHI) are ranked highest for identifying the best model with the average error (that is, difference between the GDT_TS of the selected model and the best GDT_TS) around 5 GDT_TS units. The individual ranking of these methods depends on the evaluation criteria and according to the assessment paper ${ }^{52}$ the difference between the top methods is not significant. The best consensus and quasi-single methods are only marginally worse than the pure single methods using these criteria. However, this is a significant progress in single model method performance since last CASP.

\section{2 | Distinguishing good models from bad}

From Figure 5 of the accompanying paper ${ }^{52}$ it is clear that the best approaches at detecting the top ranked models according to GDT_TS use consensus or quasi-single methods and combine them with single model approaches. The top 3 methods (Wallner, Pcomb-domain, and ModFOLD6_rank) are using the single model method ProQ2 as part of their scoring. Wallner and Pcomb-domain scores are weighted sums of ProQ2 and Pcons scores, while ModFOLD6 rank uses ProQ2 together with many other scores. Although such methods are statistically better $^{52}$ the much simpler pure consensus methods Pcons and ModFOLDclust2 are not far behind ranked sixth and ninth when using Sscore and even better than ModFOLD6 when using IDDT.

\section{3 | Ranking of models}

The ability of methods to rank the top models for each target was evaluated using the per target correlation, that is, the correlation of estimated and observed accuracy for each target. In Figure 3, the distribution of per target correlation for the all methods studied here (see Table 1) and the 3 different model accuracy estimation measures (IDDT, CAD, and GDT_TS) are shown. The distributions are sorted by the median. It can be seen that the individual rankings of the methods are quite different depending on the accuracy measure is used. When using GDT_TS, ${ }^{53}$ consensus and quasi-single based methods clearly outperform the single model accuracy estimation methods. In contrast when using $C A D^{54}$ or $I D D T^{55}$ the best correlation is obtained with ProQ3 and all the top methods are single model accuracy estimations. A similar difference in ranking can be seen in the AUC analysis on the CASP homepage (http://predictioncenter.org/casp12/qa_aucmcc.cgi). Here, ProQ3 is ranked 20th when using GDT_TS but 7th when using CAD. In contrast Pcons is ranked fourth using GDT_TS and 12th using CAD. Interestingly, it can be seen that the "pure" consensus methods (Pcons, MODFOLDClust2) show only a modest per target correlation with CAD or IDDT (Figure 3).

\section{4 | Comparison of global accuracy estimation predictions}

How similar are the model accuracy estimation scores produced by the different methods? To answer this we calculated the correlation between predicted accuracy estimates from all methods (Figure 4). The methods were then clustered using Weighted Pair Group Method Centroid (WPGMC) with the median correlation as linkage. It can be seen that all methods (except qSVMQA) that use some sort of consensus (quasi-single or consensus) are clustered. Within this group the separation is primarily not between quasi-single methods and consensus methods, but rather between the methods that primarily use consensus and those that combine the consensus score with ProQ2 (Pcomb-domain, ModFOLD6_rank, Wallner, and ModFOLD6). ModFOLD6_cor is more similar to the pure consensus methods (Pcons and ModFOLDclust2) than the other combined methods as it does not use ProQ2 global scores directly in its classification. Since the combined methods include single methods they are also more similar to all the single methods than the pure consensus methods.

Single model accuracy estimation methods show the largest performance diversity. SVMQA is the least similar to the others, being more similar to the consensus methods than to any other single model accuracy estimation method. The other 3 methods are more correlated, with the newer methods ProQ3 and MESHI showing the highest correlation. It can also be noted that in general ProQ2 is the outlier, showing the lowest correlation with the consensus methods.

When comparing the 3 different quality measurements (GDT TS, CAD, and IDDT) it can be seen that they do not correlate with each other better than the consensus methods with GDT TS, see Figure 4. The correlation between the quality measures CAD and GDT_TS is 0.88 ; while the correlation of the predicted values from the consensus methods to GDT_TS is 0.92 or higher. Although some of the problems might origin from domain division, as mentioned in the Wallner sections, it is clear that the accuracy of model quality estimation is getting 


\section{Global correlations}
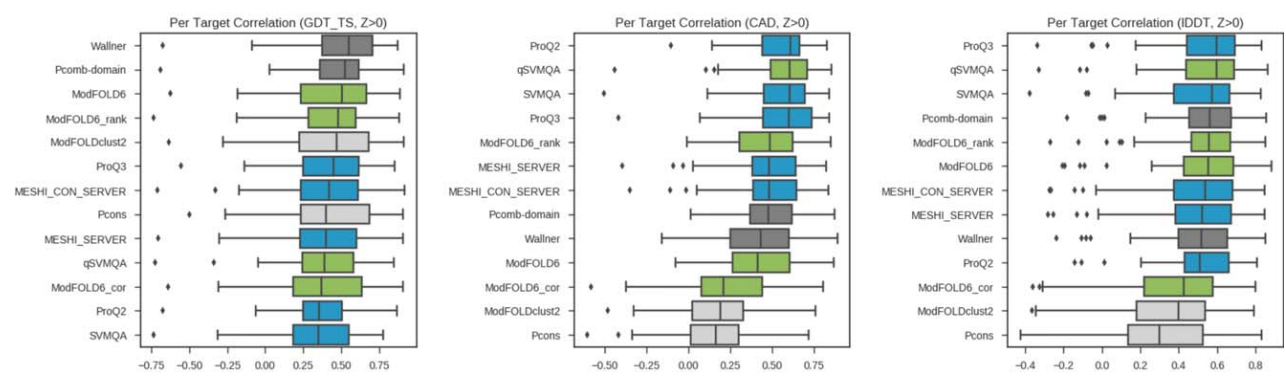

Local correlations
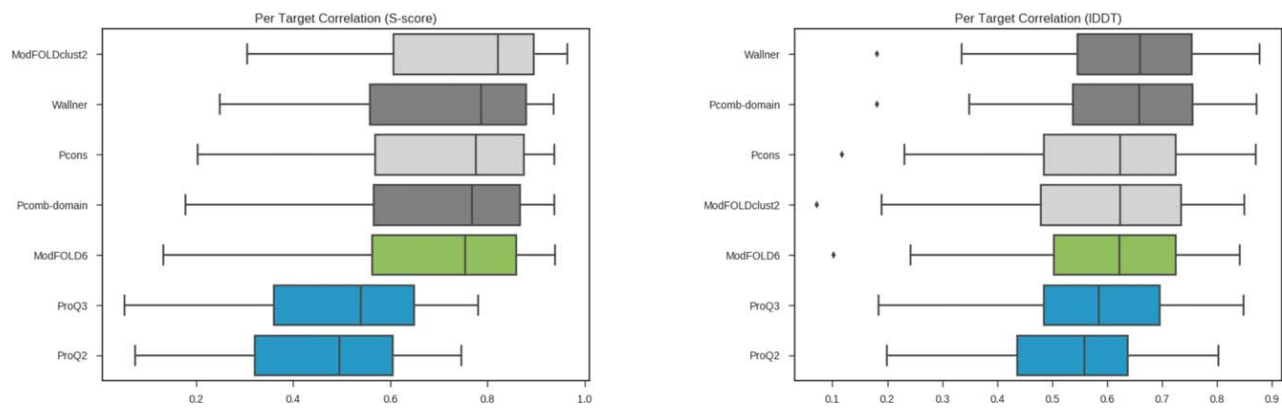

FIGURE 3 Boxplots of per target correlation for the methods presented in this article for GDT_TS, CAD, and IDDT, (A-C) global evaluations, $(D, E)$ local evaluations. To avoid bias from bad models only models with $Z>0$ are included in the global analysis. For local correlation CAD values were not available so only the distances, turned into S-scores, and IDDT values are compared. Single-model methods are colored blue, quasi green, clustering light gray and combination models dark gray. Using GDT_TS the clustering-based methods are slightly better than the single-model predictors, while this is not the case using the alternative measures CAD and IDDT. Clustering methods benefit from having low-quality models in the pool while the single model methods appear better at ranking higher quality models. For both local measures the single-model evaluation methods have lower correlation than the superposition based ones, but the difference in correlation is smaller when using IDDT [Color figure can be viewed at wileyonlinelibrary.com]

close to a point where they challenge the notion of measuring the quality of a model given a known native structure.

\section{5 | Local accuracy estimation in CASP12}

In terms of estimation of local accuracy, the best performance is obtained by the pure consensus methods followed by quasi-single model approaches. $^{52}$ In Figure 5 , a heat map of the correlation between all local predictions by the methods discussed in this article is shown. Unfortunately, of the single predictors evaluated here only ProQ2 and ProQ3 produce local predictions, nevertheless the trend is similar as for the global methods. All the consensus and quasi-single methods provide very similar accuracy estimates, while the 2 single model methods are outliers. It is clear from this analysis that the consensus methods correlate better with S-score (cc 0.85) than with IDDT (cc 0.77). As the consensus methods are based on superposition algorithms, similar to those used when calculating the $\mathrm{S}$-score, this might not come as a surprise. Interestingly both ProQ2 and ProQ3 correlate better with IDDT ( $c c \sim 0.71$ ) than with S-score (cc $\sim 0.65$ ). It can also be noted that ProQ3 correlates better than ProQ2 with both IDDT and S-score. This highlights the improvements achieved in single model quality estimates since CASP11.

\section{4 | DISCUSSION}

For the first time single model quality estimators can challenge the consensus-based methods when it comes to ranking of targets. However, the consensus-based estimators are still superior when it comes to local quality estimation, at least when using the CASP defined criteria. Below we will continue the CASP style of presentations by summarizing what each group learned during CASP12.

\section{1 | What the Elofsson group learned}

An interesting trend in CASP12 is that ProQ3 is better than our consensus method, Pcons, at picking up the best model, see EMA 


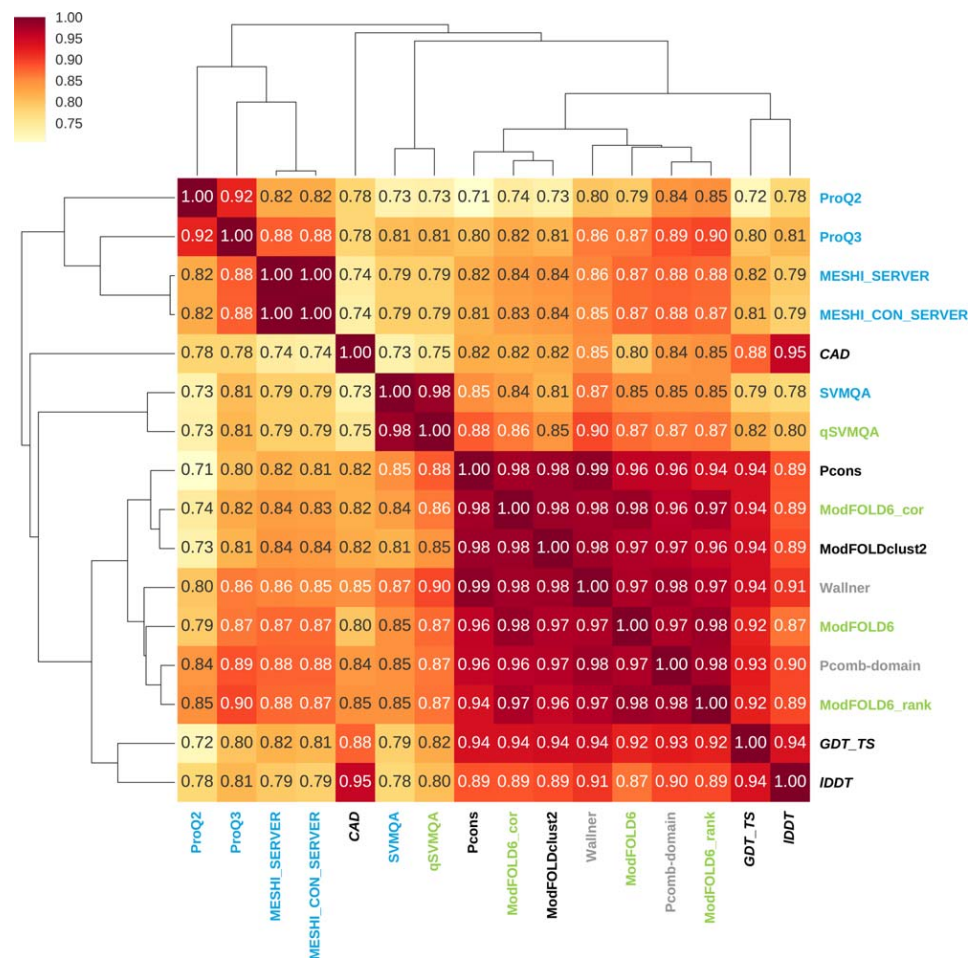

FIGURE 4 Pairwise correlations between predicted global accuracy scores from different methods and actual accuracy scores according to 3 measures. The methods are clustered hierarchically using WPGMC algorithm with the median correlation as similarity measure. Methods are colored as follows. Dark gray, pure consensus methods; light gray, combined single/consensus methods; green, quasi-single methods; and blue pure single methods. It can be noted that (i) both quasi, pure, and combined consensus methods are very similar (cc $>0.94$ ), while the single model quality methods are more different ( $\mathrm{cc}<0.90$ between the groups). ProQ2 is the real outlier having a $\mathrm{cc}<0.82$ to most methods. Interestingly ProQ2 and ProQ3 are less similar to each other than any pair of consensus-based methods. It can also be noted that the combined methods are more similar to the single-model methods than the pure consensus methods (Pcons, ModFOLDClust2)

assessment paper. ${ }^{52}$ In earlier CASPs this was not the case and until CASP10 it was clear that consensus-based methods were superior even in this aspect. We do believe that the main reason for this is that single model accuracy estimation methods have improved in the last few years.

However, consensus-based methods such as Pcons are still superior at separating correct and incorrect models. ${ }^{52}$ Interestingly, when using CAD, ProQ3 performs slightly better than Pcons even on this measure, see Figure 3, indicating that some part of the superior performance of consensus methods might be due to multi-domain properties of the targets or the choice of target function.

One issue at CASP is that the definition of the target function for local prediction used in CASP might not be ideal. The goal is to predict the error in distance for a particular residue. However, this is dependent on the superposition used, which can be problematic for multidomain targets. It could therefore be useful in future CASPs to consider changing the target function to one of the nonsuperposition-based quality evaluations, such as CAD or IDDT. The stated goal in CASP12 is to predict the distance after superposition and for this consensus methods are better. However, the performance of ProQ3 is getting closer to the performance of the consensus methods when using IDDT for model quality estimation, see Figures 3 and 5 .

\subsection{What the Keasar group learned}

The major rationale behind the design of MESHI-score pipeline (Figure 1 ) is to keep the feature set painlessly extendable. To this end we employed an ensemble-learning scheme, in which the feature selection is part of the training of each predictor (ie, ensemble member). This way each feature has a "fair chance" to be included in some of the predictors and provide its unique contribution to the overall score. Overfitting at the single predictor level is avoided by restricting the number of selected features. Combining the set of predictor scores to form the single ensemble score (MESHI-score) does not require any adjustable 


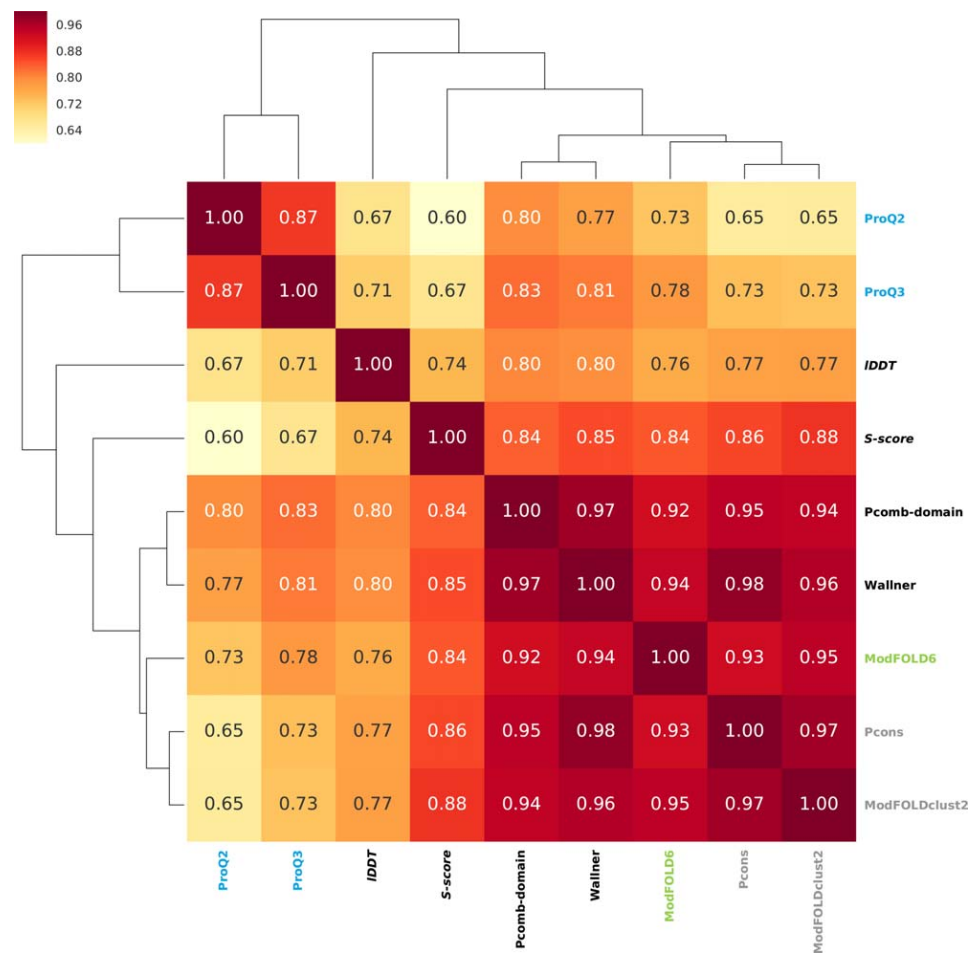

FIGURE 5 Pairwise correlation between local predicted S-scores calculated from the predicted distance using S-score formula (see above) with $d_{0}=5$ and local IDDT values (unfortunately local CAD scores were not available). Only methods that predicted local quality are included. As the ModFOLD6 methods only differ in their global scores and provide identical local estimates they were all represented by the ModFOLD6 method. Methods are colored as follows. Dark gray, pure consensus methods; light gray, combined single/consensus methods; green, quasi-single methods; and blue pure single methods [Color figure can be viewed at wileyonlinelibrary.com]

parameters and thus, does not introduce overfitting at the ensemble level. In this experiment we put to test the modularity of our ensemble learning approach. Indeed, in this experiment we were able to get better results than before, simply by adding more features to the same machinery, with neither considerable computational burden nor overfitting. This encourages us to work on the development and adoption of more informative features.

In CASP12 we also tested MESHI-score-con for the first time, and its performance was a bit superior to that of MESHI-score. We take this as a proof of concept and wish to extend it in 2 directions: have a data-driven less restricted definition of the neighbors set, and apply the same idea also to decoys of high score. High scores to 2 dissimilar decoys must imply that at least one of them (often both) is wrong.

\subsection{What the Lee group learned}

According to the CASP12 assessment, SVMQA is one of the best methods for selecting good quality models from a set of given decoys in terms of GDT Loss. The newly implemented features (5 potential energy-based terms and consistency-based terms) a systematic benchmarking approach on the selection of the final set of features, the optimization of machine learning parameters on a balanced training and testing dataset, and the usage of 2 separate predictors made SVMQA to perform significantly better than our old method used in CASP11 (RFMQA) when benchmarked on CASP11 targets. Additionally, SVMQA made valuable contribution to our tertiary structure prediction server (GOAL) and human predictors (LEE and LEEab) of CASP12 in terms of model selection. In terms of the model selection, SVMQA performed well. However, in terms of assigning proper absolute global accuracy value to a model it didn't perform as desired. ${ }^{52} \mathrm{We}$ believe that one way to improve on estimating the absolute score of a given model is to consider other types of objective functions to train separately for absolute global accuracy, which is one of the goals that we should work on for the next CASP.

\subsection{What the McGuffin group learned}

The ModFOLD6 series of methods (ModFOLD6, ModFOLD6_rank and ModFOLD6_cor) perform particularly well in terms of assigning absolute global accuracy values. As expected the ModFOLD6_cor variant is 


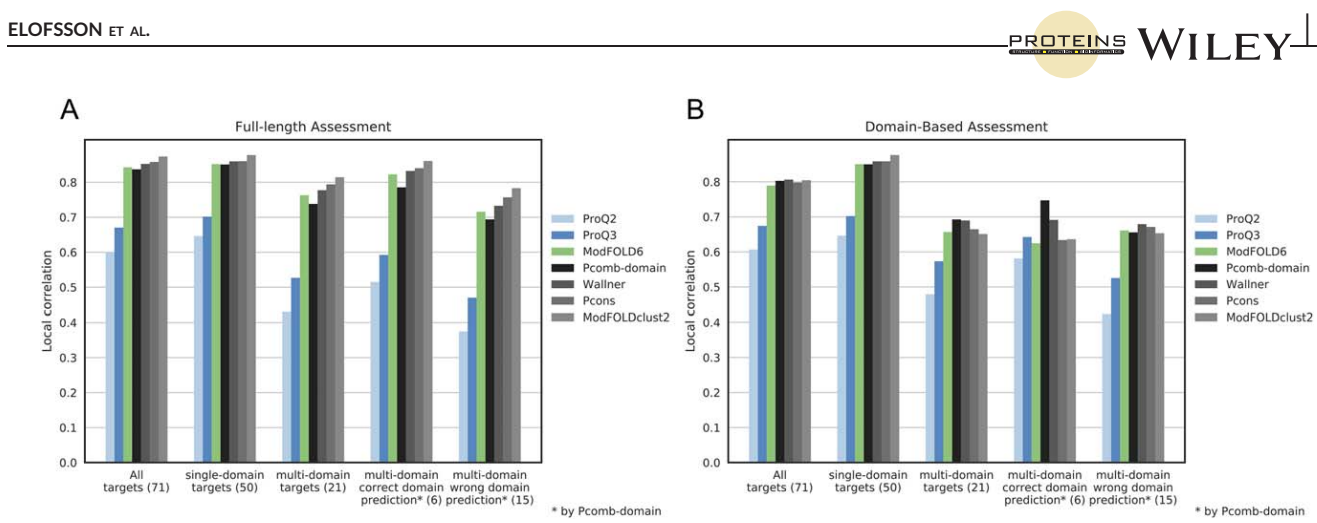

FIGURE 6 Per residue correlation of local predicted S-scores transformed using $S$-score formula with $d_{0}=5$; based on full-length targets (A) and target domains (B) for selected methods and targets divided into multi and single domain targets. For full-length assessment methods based on superposition are superior. However, Pcomb-domain performs better than other methods when (and only when) it gets the domain prediction correct [Color figure can be viewed at wileyonlinelibrary.com]

the best of these as it was optimized for this task. The ModFOLD6 series of methods also perform competitively with clustering approaches for differentiating between good and bad models; the ModFOLD6_rank method being the best of these, which is only outperformed by 2 clustering groups (Wallner and Pcomb-domain). Furthermore, as we anticipated, the ModFOLD6_rank variant is better at selecting the top models than the ModFOLD6 and ModFOLD6_cor variants; however, it is outperformed by the latest pure-single mode methods. Overall, in terms of global scores, the ModFOLD6 variants rank within the top 3 methods for nearly every global benchmark according to IDDT and CAD scores, as well as ranking within the top 10 according to other scores.

It is gratifying to see progress in CASP12 from many groups in both pure- and quasi-single model approaches to estimate model accuracy. However, it is also clear there is still room for improvement of our methods. For instance, we are outperformed in terms of model selection by the newer pure single model methods. Further integration of methods is probably needed. Different methods are clearly better suited for different aspects of model accuracy estimation, therefore all approaches to the problem are still important to pursue. Perhaps the most difficult problem faced by all groups is how to optimize a global score for all aspects of model accuracy estimation, as there seems to be no one-size-fits-all solution presently. One potential solution to this might be to use a deep learning approach that outputs multiple scores depending on the intended use case. A global score for ranking models on a per-target basis, irrespective of the observed model-target similarity scores, is clearly very useful, if it can consistently select the better models. On the other hand a global score that can produce a near 1:1 mapping between predicted and observed scores, that is consistent across all targets, will allow us to assign accurate confidence scores to individual models (which is arguably more useful to an experimentalist than a top ranked, but nevertheless poor quality, model). Of course, as model accuracy estimation methods continue to improve and approach perfect optimization for each use case, eventually the scores may converge on a single answer.

\section{5 | What the Wallner group learned}

Wallner and Pcomb-domain were the 2 best methods for differentiating between good and bad models (see assessment paper ${ }^{52}$ ). We were disappointed with the performance of Pcomb-domain, since in our benchmarks before CASP it performed significantly better than Wallner. However, the true advantage of Pcomb-domain can only be seen if the assessment is based on domains or on using superposition independent evaluation measures like IDDT ${ }^{55}$ and CAD-score. ${ }^{54,55}$ We calculated the per-residue correlation of local predicted S-scores transformed using S-score formula (see above) based on either full-length target or target domains, see Figure 6. For full-length assessment, methods based on global structural superposition (Wallner, Pcons, and ModFOLDclust2) for single domains are indeed superior. Also the performance based on multi-domain targets seems to be better for these methods (Figure 6A). However, the reason for this seemingly good performance for multidomain targets is an artifact of the full-length assessment on multidomain proteins that will only superimpose on one domain, if the domain-domain orientation is wrong. This superposition will assign high quality scores to the residues from one domain (usually the larger), and relatively low quality scores to the residues from the other domain. This effect accentuates the performance for prediction methods using global superposition, which will also predict high quality scores for one domain and low scores for the others. If instead performance is measured using the official CASP domain definitions, this artifact can be avoided, and then Pcomb-domain performs better for multi-domain targets, and better than other methods when it uses a correct domain prediction (Figure 6B). Unfortunately, correct domain prediction was only achieved for 6 out of 21 multi-domain targets. Still, it pinpoints that there should be clear room for improving Pcomb-domain by improving the domain prediction algorithm.

\section{5 | CONCLUSIONS}

It is our belief that the most important insight from the QA groups in CASP12 is the progress in single model accuracy estimations. 3 new 
methods, SVMQA, MESHI, and ProQ3, are all better than the best single model method in CASP11 (ProQ2). These methods are also bette at selecting the top-ranked model compared to consensus-based methods. However, quasi-single model method and consensus methods are still superior when it comes to distinguishing correct and incorrect models as well as for local predictions. In those targets that have a wide spread of quality there is a clear distinction between the correlations of single and consensus methods with the later performing better. These are typically subunit of protein complexes, for which templates are available. Here, estimating the accuracy of a single model might not make sense without taking the entire complex into account. In CASP12 this is most dramatic for target T0865, where correlations for consensus-based methods are high and correlations for all single mode methods are negative. By comparing the predictions to each other it is seen that all consensus and quasi-single methods actually are very similar, while there is larger variation between the single methods, hence combining them may provide additional value in the future.

During this evaluation we noted issues for multi-domain targets where the individual domains are correct but not their relative arrangement. Here, the GDT_TS score (and any superposition-based score) is based on the superposition of the largest domain. This causes problems when the evaluation is not domain based. For model quality estimations the problem is most notable when evaluating local QAs. It could therefore be useful, in future CASPs, to use $C A D^{54}$ or IDDT ${ }^{55}$ to evaluate the quality of a model without using domain division. We do also notice that single model estimation methods perform better when assessed with CAD or IDDT see Figures 3 and 5 .

\section{ACKNOWLEDGMENTS}

First of all we are very grateful to all the work done by the late Prof. Anna Tramontano who has been fundamental for CASP. Her contribution will never be forgotten. We also thank Dr Andriy Kryshtafovych for his evaluation of our methods in CASP including providing additional data for our evaluations. We also thank the rest of the CASP team for their efforts with CASP12. Finally we acknowledge all the CASP participants who contributed with predictions that we could evaluate.

\section{ORCID}

Arne Elofsson (iD http://orcid.org/0000-0002-7115-9751 Jooyoung Lee (D) http://orcid.org/0000-0003-2654-712X Liam J. McGuffin (1D) http://orcid.org/0000-0003-4501-4767 Björn Wallner (iD) http://orcid.org/0000-0002-3772-8279

\section{REFERENCES}

[1] Novotný J, Bruccoleri R, Karplus M. An analysis of incorrectly folded protein models. Implications for structure predictions. J Mol Biol. 1984;177:787-818.

[2] Samudrala R, Levitt M. Decoys 'R' Us: a database of incorrect conformations to improve protein structure prediction. Protein Sci. 2000;9:1399-1401.
[3] Lüthy R, Bowie JU, Eisenberg D. Assessment of protein models with three-dimensional profiles. Nature. 1992;356:83-85.

[4] Domingues FS, et al. Sustained performance of knowledge-based potentials in fold recognition. Proteins. 1999;Suppl 3:112-120.

[5] Sippl MJ, Lackner P, Domingues FS, Koppensteiner WA. An attempt to analyse progress in fold recognition from CASP1 to CASP3. Proteins. 1999:Suppl 3:226-230.

[6] Wallner B, Elofsson A. Can correct protein models be identified? Protein Sci. 2003;12:1073-1086.

[7] Benkert P, Tosatto SCE, Schomburg D. QMEAN: a comprehensive scoring function for model quality assessment. Proteins. 2008;71: 261-277.

[8] Kryshtafovych A, Barbato A, Monastyrskyy B, Fidelis K, Schwede T, Tramontano A. Methods of model accuracy estimation can help selecting the best models from decoy sets: assessment of model accuracy estimations in CASP11. Proteins. 2016;84 Suppl 1:349-369.

[9] Bujnicki JM, Elofsson A, Fischer D, Rychlewski L. Structure prediction meta server. Bioinformatics. 2001:17:750-751.

[10] Fischer D, et al. CAFASP2: the second critical assessment of fully automated structure prediction methods. Proteins. 2001; Suppl 5: 171-183.

[11] Lundström J, Rychlewski L, Bujnicki J, Elofsson A. Pcons: a neuralnetwork-based consensus predictor that improves fold recognition. Protein Sci. 2001;10:2354-2362.

[12] Ginalski K, Elofsson A, Fischer D, Rychlewski L. 3D-Jury: a simple approach to improve protein structure predictions. Bioinformatics. 2003;19:1015-1018.

[13] Wallner B, Elofsson A. Prediction of global and local model quality in CASP7 using Pcons and ProQ. Proteins. 2007:69 Suppl 8:184193

[14] Cozzetto D, Kryshtafovych A, Ceriani M, Tramontano A. Assessment of predictions in the model quality assessment category. Proteins. 2007;69(Suppl 8):175-183.

[15] McGuffin L, Buenavista MT, Roche DB. The ModFOLD4 server for the quality assessment of $3 \mathrm{D}$ protein models. Nucleic Acids Res. 2013;41:W368-W372.

[16] Maghrabi AHA, McGuffin LJ. ModFOLD6: an accurate web server for the global and local quality estimation of 3D protein models. Nucleic Acids Res. 2017;doi:10.1093/nar/gkx332. Epub ahead of publication.

[17] Uziela K, Shu N, Wallner B, Elofsson A. ProQ3: improved model quality assessments using Rosetta energy terms. Sci Rep. 2016;6: 33509

[18] Uziela K, Menéndez Hurtado D, Shu N, Wallner B, Elofsson A. ProQ3D: improved model quality assessments using deep learning. Bioinformatics. 2017:33:1578-1580.

[19] Wallner B, Elofsson A. Pcons5: combining consensus, structural evaluation and fold recognition scores. Bioinformatics 2005;21:4248-4254.

[20] Wallner B, Elofsson A. Identification of correct regions in protein models using structural, alignment, and consensus information. Protein Sci. 2006;15:900-913.

[21] Ray A, Lindahl E, Wallner B. Improved model quality assessment using ProQ2. BMC Bioinformatics. 2012;13:224

[22] Levitt $M$, Gerstein M. A unified statistical framework for sequence comparison and structure comparison. Proc Natl Acad Sci U S A. 1998;95:5913-5920.

[23] Cristobal S, Zemla A, Fischer D, Rychlewski L, Elofsson A. A study of quality measures for protein threading models. BMC Bioinformatics. 2001;2:5 
[24] Skwark MJ, Elofsson A. PconsD: ultra rapid, accurate model quality assessment for protein structure prediction. Bioinformatics. 2013;29: 1817-1818.

[25] Mirzaei S, Sidi T, Keasar C, Crivelli S. Purely structural protein scoring functions using support vector machine and ensemble learning. IEEE/ACM Trans. Comput. Biol. Bioinform. 2016;doi:10.1109/ TCBB.2016.2602269

[26] Wang Q, Canutescu AA, Dunbrack RL. Jr., SCWRL and MollDE: computer programs for side-chain conformation prediction and homology modeling. Nat Protoc. 2008;3:1832-1847.

[27] Krivov GG, Shapovalov MV, Dunbrack RL. Jr., Improved prediction of protein side-chain conformations with SCWRL4. Proteins. 2009; 77:778-795.

[28] Summa CM, Levitt M. Near-native structure refinement using in vacuo energy minimization. Proc Natl Acad Sci U S A. 2007;104 3177-3182.

[29] Samudrala R, Moult J. An all-atom distance-dependent conditional probability discriminatory function for protein structure prediction. I Mol Biol. 1998;275:895-916

[30] Zhou H, Skolnick J. GOAP: a generalized orientation-dependent, allatom statistical potential for protein structure prediction. Biophys. J. 2011:101:2043-2052.

[31] Jones DT. Protein secondary structure prediction based on position-specific scoring matrices. J Mol Biol. 1999;292:195-202.

[32] Amir E-AD, Kalisman N, Keasar C. Differentiable, multi-dimensional, knowledge-based energy terms for torsion angle probabilities and propensities. Proteins. 2008;72:62-73.

[33] Levy-Moonshine A, Amir E-AD, Keasar C. Enhancement of betasheet assembly by cooperative hydrogen bonds potential. Bioinformatics. 2009:25:2639-2645.

[34] Manavalan B, Lee J. SVMQA: support-vector-machine-based protein single-model quality assessment. Bioinformatics. 2017:33:24962503. doi:10.1093/bioinformatics/bt×222

[35] Manavalan B, Lee J, Lee J. Random forest-based protein mode quality assessment (RFMQA) using structural features and potential energy terms. PLoS One. 2014;9:e106542

36] Jones DT, Ward JJ. Prediction of disordered regions in protein from position specific score matrices. Proteins. 2003:53(Suppl 6): 573-578.

37] Jones DT, Singh T, Kosciolek T, Tetchner S. MetaPSICOV: combining coevolution methods for accurate prediction of contacts and long range hydrogen bonding in proteins. Bioinformatics. 2015;31 999-1006.

[38] McGuffin LJ. The ModFOLD server for the quality assessment of protein structural models. Bioinformatics. 2008;24:586-587.

[39] McGuffin LJ. Prediction of global and local model quality in CASP8 using the ModFOLD server. Proteins. 2009;77(Suppl 9):185-190.

[40] Uziela K, Wallner B. ProQ2: estimation of model accuracy implemented in Rosetta. Bioinformatics. 2016;32:1411-1413.
[41] McGuffin L, Roche DB. Automated tertiary structure prediction with accurate local model quality assessment using the IntFOLD-TS method. Proteins. 2011;79(Suppl 10):137-146

[42] Buenavista MT, Roche DB, McGuffin LJ. Improvement of 3D protein models using multiple templates guided by single-template model quality assessment. Bioinformatics. 2012;28:1851-1857.

[43] Roche DB, Buenavista MT, Tetchner SJ, McGuffin LJ. The IntFOLD server: an integrated web resource for protein fold recognition, 3D model quality assessment, intrinsic disorder prediction, domain prediction and ligand binding site prediction. Nucleic Acids Res. 2011;39: W171-W176.

[44] McGuffin $\sqcup$, Atkins JD, Salehe BR, Shuid AN, Roche DB. IntFOLD: an integrated server for modelling protein structures and functions from amino acid sequences: figure 1. Nucleic Acids Res. 2015;43:W169-W173.

[45] Haas J, et al. The Protein Model Portal-a comprehensive resource for protein structure and model information. Database. 2013:2013:bat031.

[46] McGuffin $\sqcup$, Roche DB. Rapid model quality assessment for protein structure predictions using the comparison of multiple models without structural alignments. Bioinformatics. 2010;26:182-188.

[47] Kryshtafovych A, Fidelis K, Tramontano A. Evaluation of model quality predictions in CASP9. Proteins. 2011;79(Suppl 10):91-106.

[48] Kryshtafovych A, et al. Assessment of the assessment: evaluation of the model quality estimates in CASP10. Proteins. 2014;82(Suppl 2): 112-126.

[49] Chivian D, et al. Automated prediction of CASP-5 structures using the Robetta server. Proteins. 2003;53(Suppl 6):524-533.

[50] McGuffin $\sqcup$, et al. Accurate template-based modeling in CASP12 using the IntFOLD4-TS, ModFOLD6, and ReFOLD methods. Proteins. 2017; doi:10.1002/prot.25360. Epub ahead of publication.

[51] Larsson P, Skwark MJ, Wallner B, Elofsson A. Assessment of global and local model quality in CASP8 using Pcons and ProQ. Proteins. 2009;77(Suppl 9):167-172.

[52] Kryshtafovych A, Monastyrskyy B, Fidelis K, Schwede T, Tramontano A. Assessment of model accuracy estimations in CASP12. Proteins. 2017; doi:10.1002/prot.25371. Epub ahead of publication.

[53] Zemla A. LGA: a method for finding 3D similarities in protein structures. Nucleic Acids Res. 2003;31:3370-3374.

[54] Olechnovič K, Kulberkytè E, Venclovas C. CAD-score: a new contact area difference-based function for evaluation of protein structural models. Proteins. 2013;81:149-162.

[55] Mariani V, Biasini M, Barbato A, Schwede T. IDDT: a local superposition-free score for comparing protein structures and models using distance difference tests. Bioinformatics. 2013;29:2722-2728.

How to cite this article: Elofsson A, Joo K, Keasar C, et al. Methods for estimation of model accuracy in CASP12. Proteins. 2017;00:000-000. https://doi.org/10.1002/prot.25395 



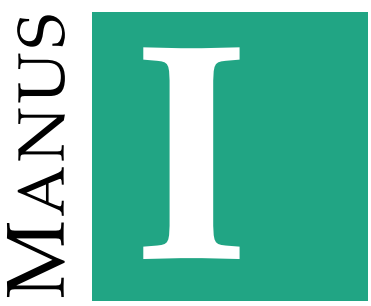

Robert Pilstål and Björn Wallner. "Improvements in Protein Model Quality Assessment from Automated Domain Partitioning using Spectral Clustering". Apr. 2018 



\section{DAQA: Improvements in Protein} Model Quality Assessment from Automated Domain Partitioning using Spectral Clustering.

*For correspondence:
robert.pilstal@liu.se (KRP); bjorn.wallner@liu.se (BW)

Present address: ${ }^{5} \mathrm{FFM}$, Linköpings universitet, SE-581 83 Linköping

\author{
Karl Robert Pilstål ${ }^{1 *}$ and Björn Wallner ${ }^{1 \S^{\star}}$ \\ ${ }^{1}$ Department of Physics, Chemistry and Biology - Division of Bioinformatics
}

\begin{abstract}
The protein folding problem discuss the relationship between protein primary and tertiary structure, or the sequence to form transformation. Understanding protein structures enable their functional analysis, reveal mechanisms and promises epidemiological relevations. Numerous methods for protein structure prediction exists, but none with a definitive answer to the folding problem; therefore multiple methods of protein structure quality assessment is used to score plausible protein structure models to help sieve through these decoys generated from softwares. The most promising quality assessment methodologies available are those using consensus, where quality is estimated using clustering methods of an ensemble of decoys. Our approach to quality assessment use automated protein domain partitioning to divide and conquer the problem, forming a consensus for each separated part. In this way we alleviate some of the issues in superposition of multi-domain protein decoys and can better estimate their quality. The method compares favorable with the best methods in the estimation of model accuracy category in CASP12.
\end{abstract}

\section{Introduction}

Proteins are essential building blocks of life, they execute functions necessary to the survival of our cells. Their function is didacted by their structure, thus knowing the structure is a key step in determining protein function. To this end considerable efforts have been dedicated towards experimentally determine protein structure. However, given the gap between the number of known protein sequences $(111,425 \mathrm{k}$, Mar-28, 2018) in UniProt (The UniProt Consortium, 2017) and the known protein structures (140k April 2018) in the PDB (Berman et al., 2000) is more than 795-fold there is no possibility to realistically have experimentally solve structures for every known sequence. Thus, for the vast majority of sequences computational methods that can predict the structures needs to be employed.

Many methods to predict protein structures have been developed over the years. A critical component in all of these methods is to accurately assess the quality of generated plausable models to be able to select the best possible models from several alternative models. This has given rise the to field of model quality assessment or estimation of model accuracy (EMA methods),

36 throughly benchmarked in CASP (Kryshtafovych et al., 2018). In principle, EMA methods can be divided into three groups depending on what type of information they are using for their prediction; single methods using only individual protein models, consensus methods that are using information from an ensemble of models, and hybrid methods using a combination of sing/e and consensus 
40 methods. They all have their pros and cons, the hybrid methods, using the most information have 41 the best performance, followed by the consensus and the single methods (Kryshtafovych et al., 2018;

42 Elofsson et al., 2017). The single methods, on the other hand, can be used for sampling and can be

43 used to score millions of potential models (Uziela and Wallner, 2016), and they can also be used to

44 improve the consensus methods, since they provide additional information (Buenavista et al., 2012;

45 Uziela et al., 2017; Manavalan and Lee, 2017). The consensus methods uses structural similarity

46 among a set of plausable models to rank models (Ginalski et al., 2017; Wallner and Elofsson, 2005),

47 effectively giving higher ranks to models that are more similar to the model ensemble. Despite the

48 simplicity of the consensus methods they provide outstanding performance as demonstrated in the

49 latest CASP12 (Kryshtafovych et al., 2018).

The quality estimation can be performed on two levels; global and local. The global level is estimating the accuracy of the model as a whole, while the local level estimate the accuracy of each individual residue in the model. The latter is potential more useful as it could pinpoint which regions of a model to trust and which regions that needs to improved.

Despite the general success of the consensus and hybrid methods in CASP12 (Kryshtafovych et al., 2018), there is still room for improvement. A clear bottle-neck for the consensus methods is in how the structural similarity is measured. This is usually done by comparing all models in the ensemble using a rigid-body structural superposition measures such as LGscore (Cristobal et al., 2001), S-score (Levitt and Gerstein, 2017), MaxSub (Siew et al., 2000), TMscore (Zhang and Skolnick, 2004), or GDT_TS (Zemla et al., 1999). This works fine for small single domain proteins. However, they run into problems for multi-domain proteins, where the individual domains are correctly model, but their relative orientation is incorrect. In this case, the consensus will be performed on the domain which is most similar, and any additional domains will effectively be assigned very poor quality.

The superposition problem can to some extent be solved by using a superposition independent or contact-based measures such as LDDT (Mariani et al., 2017) and CAD-score (Olechnovič et al.,

6 2013). There are some consensus methods that are using contacts, but they were mainly developed

for speed rather than to solve the multi-domain protein problem (McGuffin and Roche, 2010;

68 Skwark and Elofsson, 2013), and potential problem with focusing on local contact features is that

9 these methods might miss the global feature differences, e.g. a long helix in a wrong position will still have many local contact features correct.

Here, describe Pcons Bipart, a combination of the consensus method Pcons (Wallner and Elofsson, 2005), one of the top-performing pure consensus methods in the latest CASP12 (Kryshtafovych et al., 2018; Elofsson et al., 2017) with an automatic partitioner that automatically partition the protein into consensus evaluation units before analysing using Pcons.

\section{Results}

To improve consensus prediction for multi-domain proteins, we developed a method that used to the well-established consensus method Pcons (Wallner and Elofsson, 2005) to automatically partition the protein into domains using spectral clustering (Figure 1). In short, the method takes an ensemble of models as input (Figure 1a), and runs an initial consensus prediction using Pcons (vanilla, Figure $1 \mathrm{~b}$ ), generating a global scoring vector over the ensemble. A tensor of distance matrices is formed by generating a distance matrix for each model in a subset of the ensemble 22 (TENSOR FORMATION, Figure 1c). This tensor is then used as input to BiPART together with the initial weight vector from Pcons. Pcons is then executed on each of two domains (Figure $1 \mathrm{~d}$ ), as 4 defined by the ignore residue masks from the PDB MASKER that converts the domain definition 5 provided by the domain partitioner (BiPART, in Figure 1 e) based on spectral clustering, allowing assessment on separate domains in parallel. The resulting quality scores are then combined into 7 an assessment of the complete models (BiPART, in Figure $1 \mathrm{f}$ ). In the following evaluation of quality

88 assessment we compare the assessments of Pcons (vanilla) and Pcons+BiPART (bipart) that are

89 generated in the parts of the method process encircled by the yellow box in Figure 1 over the full 


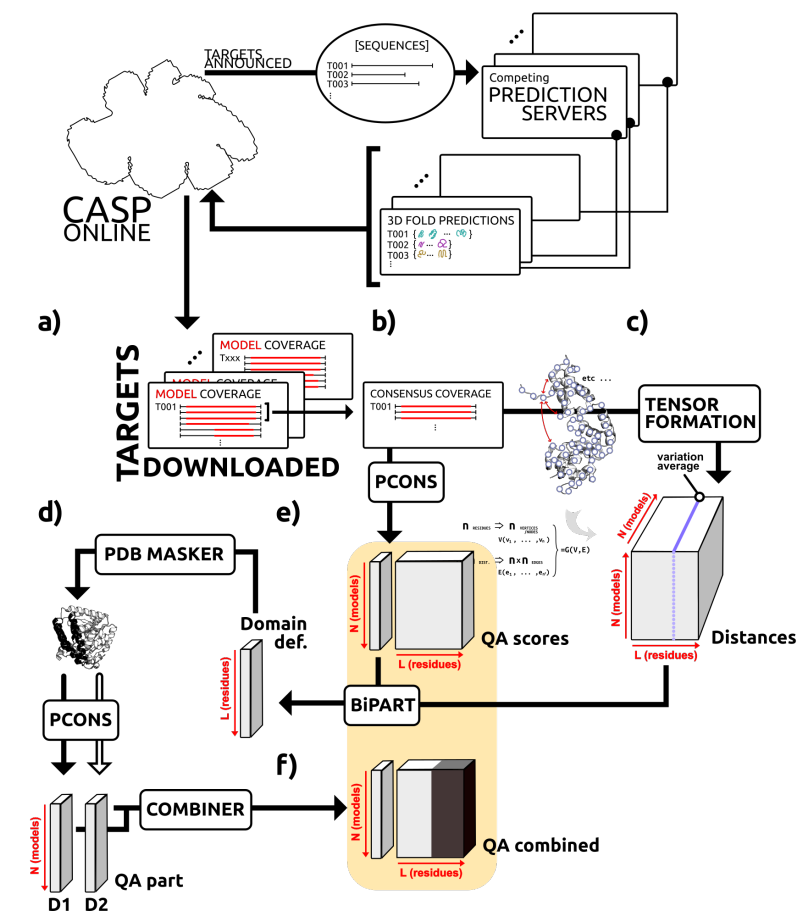

Figure 1. Overview of Pcons Bipart algorithm.

Figure 1-source data 1. from: 2018-03-27 Tue Resurser DAQA metodbeskrivning

90 model ensembles downloaded (Figure 1a). For more detail on the domain partitioner, and the other constituents, see Methods.

92 The domain partitioner generates a binary domain definitions for each target. This domain

93 definition is not based on single cut in the sequence, but rather on the local consensus in model

94 ensemble for the target. Thus, each domain could in principle consists of a number of segments,

95 i.e a range of consecutive residues. However, in most cases domains consist of a single segment

96 effectively splitting the target in two parts along its sequence (Figure 2), where the larger of the two

97 domains is roughly $50 \%$ larger than the smaller in terms of number of residues. 

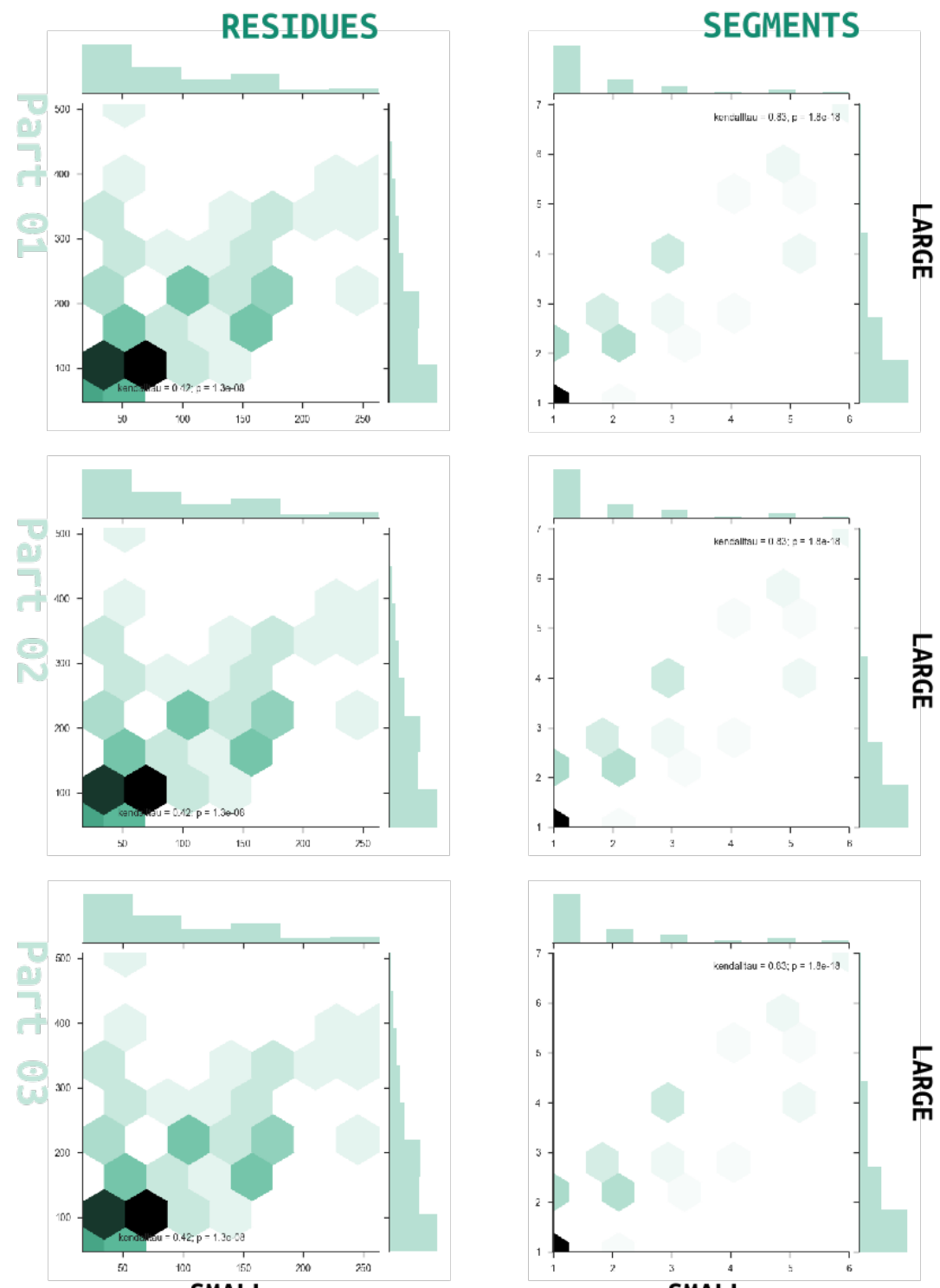

SMALL

SMALL

Figure 2. The distribution of domain sizes in number of residues and number of segments is shown in the histogram. The domains are categorized as being large and small, with the domain with larger number of residues being the larger domain.

Figure 2-source data 1. from \{2017-06-28 Wed\} Protocol - Running domainpartitioners on CASP targets 


\section{Comparing Bipart to vanilla Pcons}

99 To analyze the added value of the domain partitioner in quality assessment using consensus, vanilla 100 Pcons was compared to using Pcons and domain partitioner as outlined above (Bipart) on CASP12 101 data using local (Figure 3a) and global (Figure 3b) correlations. In the resulting correlations for 102 vanilla and Bipart, with the LDDT and LGA_SDA, we find that the vanilla method has slightly higher 103 local correlation with the superposition dependent LGA_SDA $\left(0.805\right.$ vs. $\left.0.789, \mathrm{P}<10^{-3}\right)$ while the 104 partitioned has higher correlation with the superposition independent LDDT measure (0.859 vs.

$1050.828, \mathrm{P}<10^{-3}$ ). This is to be expected since both the LGA_SDA and the vanilla method are highly 106 sensitive to domain orientation perturbations due to their use of a global superposition (Mariani et al., 2017).In addition, we also loose some global information while gaining local superposition 8 plasticity upon partitioning the models into domains. For the global correlations (Figure $3 b$ ), the partitioned is better than vanilla on LDDT $\left(0.912\right.$ Vs $\left.0.896, \mathrm{P}<10^{-} 3\right)$, while there is no difference in the correlations against the superposition dependent GDT_TS measure. Taken together it seems like the Bipart provides small improvement over vanilla Pcons.
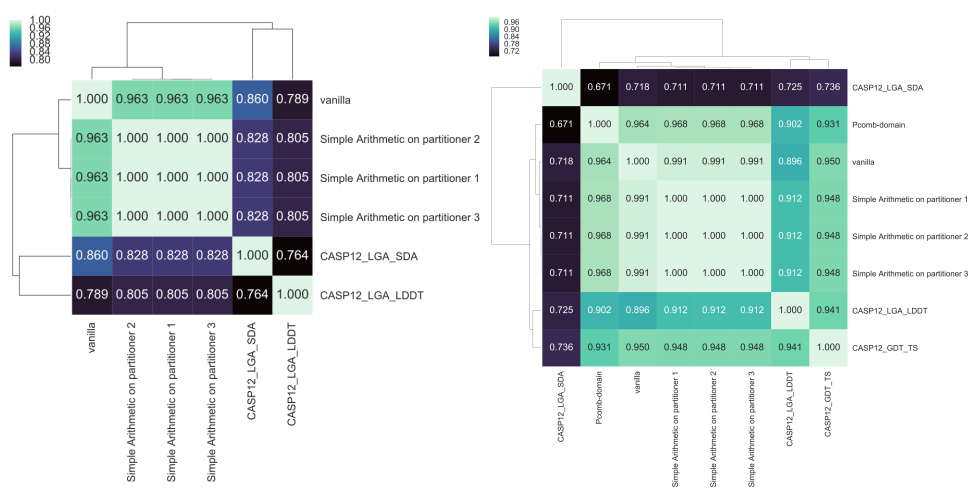

(a) Pearson's correlations coeffcient for local scores for (b) Correlations over global scores for for bipart and bipart and vanilla methods. All correlation differences vanilla. are significant with $\mathrm{P}<10^{-5}$ using Fisher's $r$ to $z^{\prime}$ transform.

Figure 3. Bipart/Vanilla comparison

Figure 3-source data 1. from: $20171112_{\text {detailedcorrelations.ipynb }}$

112 Comparing performance across targets

113 The analysis above is based on overall correlations of global and local quality measures. Here, 114 we analyze in more detail how the performance varies across the different targets by studying 115 distributions of global and local correlations calculated per target for Bipart and Vanilla (Figure 4). 116 The distributions for GDT_TS is similar in the high correlation region, while the tails in the low 117 correlation part of the distributions have a different shape (Figure 4a). In the Vanilla distribution, 118 there is an accented "hump" around 0.40 in the tail distribution, signifying a set of targets where the 119 method have significantly lower correlation with the global GDT_TS measure as compared to Bipart. 120 These targets could be related to some limitation of the Vanilla method to assess them properly such as 1) multi-domain, 2) a structurally diverse ensemble, and 3) problematic to superimpose globally. For the Bipart method, this hump is less accentuated, and is located further "up" the correlation gradient of the distribution around 0.5; indicating that the Bipart method does not suffer from the same problems as the Vanilla method for these set of targets. A similar trend is also

125 observed for the global LDDT distribution, wherr Bipart has fewer targets with correlation below 
1260.5 compared to Vanilla (Figure 4a). Also for the per target correlation for local LDDT, Bipart has 127 fewer targets with lower correlations compared to Vanilla (Figure 4b).

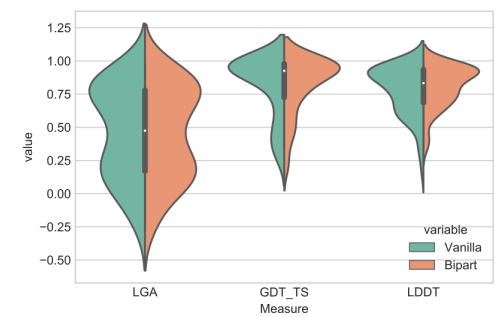

(a) Per target correlation for global measures

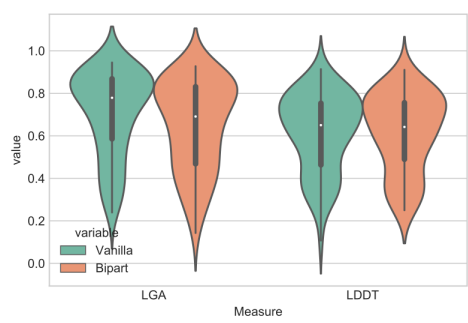

(b) Per target correlation for local measures

Figure 4. Global and local per target correlation distributions for different Vanilla and Bipart

To get an idea on how Bipart performed for different targets. The per target correlation were 129 calculated and compared to those from Vanilla and Pcomb-domain (Figure 5a), which is hybrid 130 method combining single model evaluation with consensus and partitioning that we participated 131 with in CASP12 (Elofsson et al., 2017). Targets were categorized by from their trends over correlation 132 with global GDT_TS (Figure 5a). The categories were 1) Bipart best, 2) Vanilla best, 3) Bipart better 3 but Pcomb-domain best, 4) Bipart worst, and 5) Bipart and Vanilla equal (Figure 5b-f)

In the global correlations seen for the category "Bipart best", see Figure $5 b$, one can identify four kinds of targets; those where 1) Bipart outperforms both Vanilla and Pcomb (T0859, T0878), 2) Bipart and Vanilla is outperforming Pcomb (T0862, T0893, T0904, T0907), 3) Partitioners are outperforming Vanilla (T0884, T0886, T0888) and 4) All is performing about equal (T0872, T0881, T0882).

Looking at the category "Vanilla best" on the global correlations in Figure $5 c$, we see that even though Vanilla is better than Bipart, it is so only by a small margin. This is also mainly on targets where the correlation is already high; generally over 0.8 , with only two targets below this threshold (T0866 and T0894). This might be due to that non-linearity of the correlation measure, so a Ztransform should be done first to see if this observation still holds, i.e. that the vanilla method is only marginally better than bipart when it is the best one. There are some cases where Pcomb-domain performs bad, while Bipart is performing quite OK; i.e. T0861, T0865, T0887, T0892, T0896.

In the Figure $5 \mathrm{~d}$, we observe that on the global correlation there are two cases where pcombdomain outperform markedly the other two methods T0918 and T0941. Otherwise we have that the partitioning is better with the winning method being Pcomb-domain, see targets T0863, T0864, T0897, T0914 and T0918.

In the global correlations seen for the category "Bipart worst" (Figure 5e), one can identify two kinds of targets; those where 1) Pcomb-domain is best (T0869, T0870, T0880), 2) Vanilla is best (T0890).

In the global correlations seen for the category "Bipart and Vanilla equal", see Figure 5f, we mainly find one kind of target where the correlation is almost the same for all methods. The only outliers are (T0898, T0900, T0913, T0947), for which the main trend is (best to worst) 1) Vanilla or Bipart, 2) Bipart or Vanilla, 3) Pcomb-domain. 


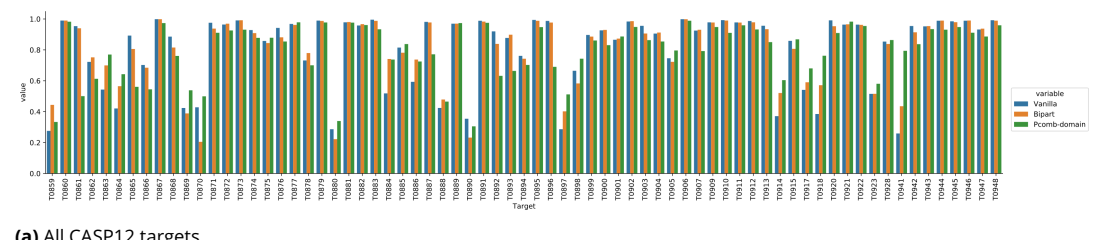

(a) All CASP12 targets

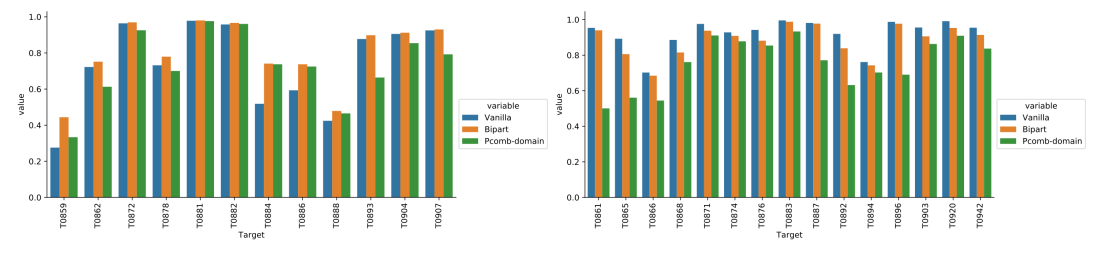

(b) Bipart best

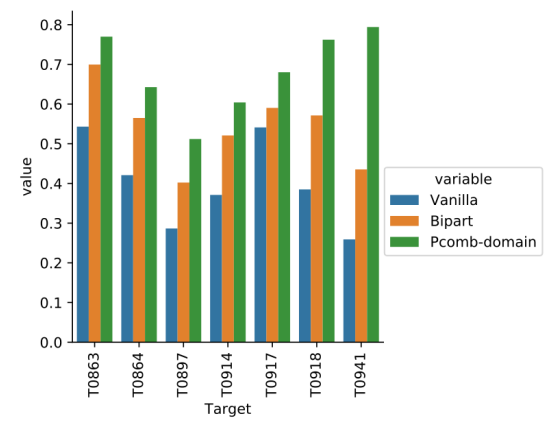

(d) Bipart better than Vanilla but Pcomb-domain best

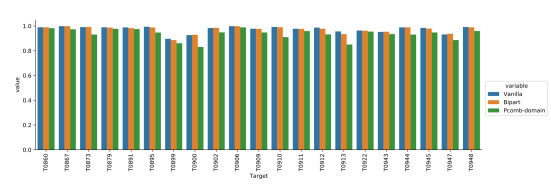

(f) Bipart eq vanilla

Figure 5. Per per target global correlation with the GDT_TS measure for Vanilla, Bipart, and Pcomb-domain on different subsets of CASP12 targets.

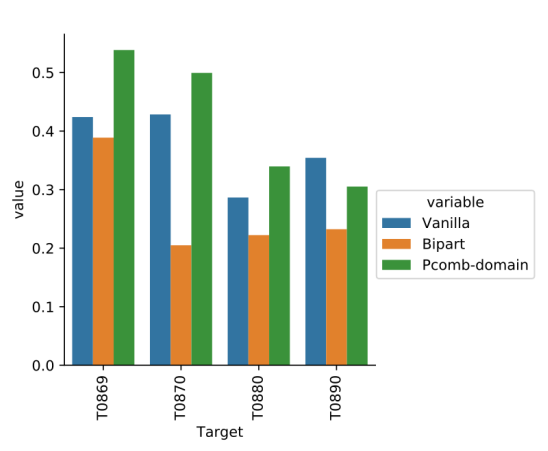

(e) Bipart worst 


\section{Interesting Examples}

158 To illustrate cases where the Bipart method is successful, the per model correlations in LDDT and 159 local LGA were calculated (Figure 6). A number of targets where the Bipart method had a better 160 distribution of model correlations with LDDT and worse with local LGA than Vanilla were selected: 161 T0861, T0866, T0893, T0903, T0907, T0920, indicated in yellow. In all of these cases, the model 162 correlations have distributions that are centered on higher correlation values on LDDT for Bipart 63 compared to Vanilla, 0.73 vs 0.48 on average; and lower model correlation on local LGA for Bipart 64 compared to Vanilla, 0.52 vs 0.84 on average (Table 1). To analyze any common denominators within 165 this group of targets, and look for clues on why partitioning the targets is useful. The structures and 66 the location of the partition are provided in Figure 7a, along with the domain definition predicted by the Bipart method in Figure $7 \mathrm{~b}$. It should be noted that the structures are the native structure, 8 which were not available at the time of prediction. The domain definition made by Bipart on the selected targets have in all cases split up the protein into two natural domains.

For T0861 the split seem to naturally split the target into two domains, however for this target there are multi-domain templates of really close homologs available. Thus, this target was evaluated as a whole and many models where almost perfect, which explains why there is actually no difference in correlation between Bipart and Vanilla for LGA (Table 1).

For T0866 the split completely ruins the LGA correlation (0.0) but increases the LDDT correlation from 0.50 to 0.70 (Table 1). For this target, only residues 38-141 were used for evaluation, because the first 37 residues and last 42 residues were not resolved in the crystal structure. Depsite the fact that the correlation was improved for LDDT, it also points to the fact that residues predicted to be disordered should not be included in the consensus evalution.

For T0893 the split is more or less identical to the offical CASP12 evaluation unit (Figure 6)b, where the helix bundle is the first domain and the rest of the structure is the second domain (D1:1-73,D2:74-242) (Table 1), explaining the increase in correlation from 0.65 for Vanilla to 0.8 for Bipart on LDDT. For the T0893 Vanilla has almost perfect has almost perfect correlaton to LGA (0.95). The reason for this high correlation is an artifact of using superposition based evaluation both as the true measure (LGA) and in the consensus analysis. The true measure will superimpose on the larger part of T0893 (D2), in effect indicating high quality for D2 and poor quality for D1. The consensus (Vanilla) will do the same and thus accumulate the effect.

Also for target T0920, the split is almost identical to the offical CASP12 evaluation unit (Figure 6)b. This drastically improves the correlation against LDDT from 0.25 for Vanilla to 0.8 for Bipart, clearly highlighting a successfull Bipart case. Again we are also seeing the same over-estimation of performance effect as for T0893 on the LGA (Table 1).

Target T0907 had three domains in the CASP12 evalution unit (D1-D3), the Bipart splits out one correctly, but merges D2 and D3, since Bipart only divides the sequence in two parts it cannot handle more than two domains in its current form (Figure 6)b. This also explains the rather weak 0.6 correlation on LDDT for Bipart, however still much higher than the 0.45 for Vanilla (Table 1 ).

\begin{tabular}{lrr|rr} 
& \multicolumn{2}{c}{ LDDT } & \multicolumn{2}{c}{ LGA } \\
Target & Bipart & Vanilla & Bipart & Vanilla \\
\hline T0861 & 0.75 & 0.60 & 0.80 & 0.80 \\
T0866 & 0.70 & 0.50 & 0.0 & 0.80 \\
T0893 & 0.80 & 0.65 & 0.75 & 0.95 \\
T0903 & 0.75 & 0.40 & 0.40 & 0.80 \\
T0907 & 0.60 & 0.45 & 0.40 & 0.75 \\
T0920 & 0.80 & 0.25 & 0.75 & 0.95 \\
\hline Average & 0.73 & 0.48 & 0.52 & 0.84 \\
\hline
\end{tabular}

Table 1. Approximate center of the per model correlation for the selected targets, from Figure 6. 


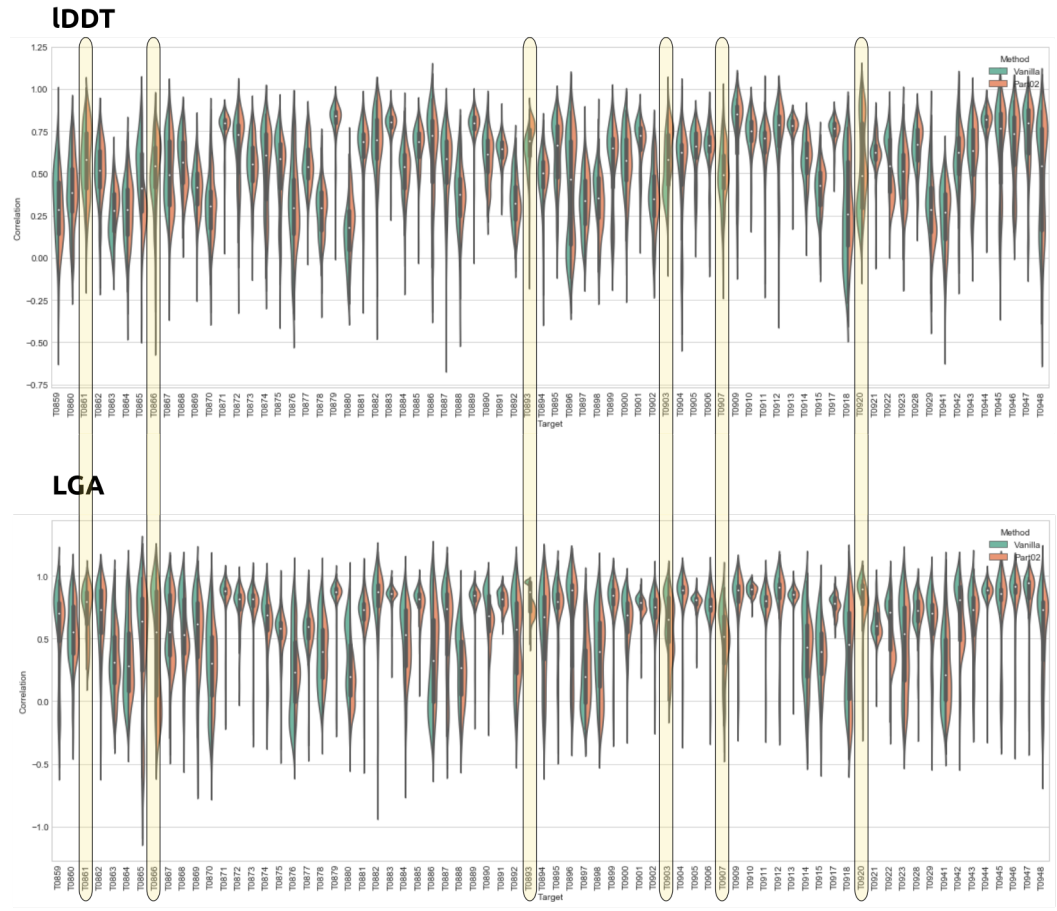

Figure 6. Selection criteria for the interesting targets. The local correlations of vanilla and bipart is against the IDDT and LGA local scores of the native structure is plotted as violin plots over each targets model ensemble. There are a number of examples where the winner in highest correlations for the vanilla and BiPART method change dramatically with respect to which correlation is plotted. These targets are highlighted in the plots and was selected for further investigation. (date: 20171109)

Figure 6-source data 1. from: 20171109 email 


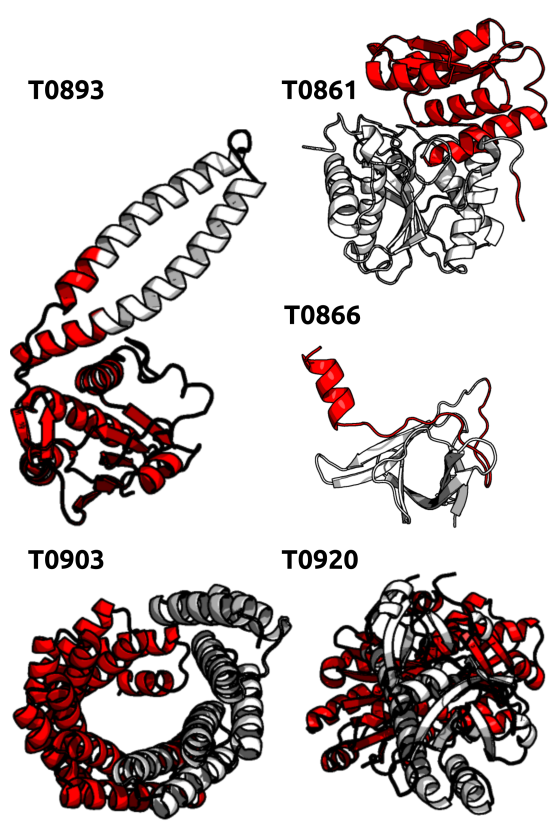

(a) Domain definitions generated by BiPART on the interesting targets with publicly available structure. One domain is colored in red, the other in white. visualizations using the PyMOL open source package DeLano (2002)

\begin{tabular}{lrrr} 
Target & Domain & Segments & CASP evaluation unit \\
\hline T0861 & 1 & $1-4,40-149$ & D1:2-313 \\
T0861 & 2 & $5-39,150-323$ & \\
T0866 & 1 & $1-122$ & D1:38-141 \\
T0866 & 2 & $123-183$ & \\
T0893 & 1 & $15-64$ & D1:1-73 \\
T0893 & 2 & $1-14,65-242$ & D2:74-242 \\
T0903 & 1 & $1-145,147-147$ & D1:15-155,168-350 \\
T0903 & 2 & $146-146,148-382$ & \\
T0907 & 1 & $1-115$ & $24-115$ \\
T0907 & 2 & $116-315$ & D2:116-198, D3:199-315 \\
T0920 & 1 & $1-322$ & D1:1-321 \\
T0920 & 2 & $323-568$ & D2:322-562 \\
\hline
\end{tabular}

(b) Domain definitions

Figure 7. Interesting target examples, T0907 is not shown in (a) since it is not yet publically released. 
195 Comparison against top-performing methods in CASP12

196 To put the results above into perspective, a comparison to the best performing model quality 197 assessment groups from CASP12 (Elofsson et al., 2017) were conducted. For global correlations to

198 LDDT (Figure 8), Bipart performs at the very top equal to the Wallner method, which was the best 199 method in CASP12. For correlations against the superposition dependent GDT_TS measure, were we 200 do expect less performance from Bipart, Bipart is still among the top group of four indistinguishable 201 methods including Bipart, vanilla, Wallner, and ModFOLDclust2. Interestingly, the best methods correlate better with GDT_TS the LDDT quality measure.

For local correlation, we chose to make the comparison pairwise on common subsets to maxi204 mize the residue overlap. Otherwise the number of residues that are included in the correlation calculations is dictated by the method that has predictions for least residues. Also here Bipart 26 methods are better than Pcomb-domain, ModFold6 and ModfoldClust2 when correlating their local scores to LDDT (Figure 9a-c), and on par with Wallner (Figure 9d). For the correlations against the local LGA_SDA score, the Bipart method performs on par with Pcomb-domain, ModFOLD6, and ModFOLDclust2 (Figure 9a-c), but is outperformed by Wallner (Figure 9d). This in agreement with the expectation that Bipart should have worse performance on the superposition dependent measure LGA_SDA. The Wallner method is not a pure consensus method, but a hybrid method that combines machine learning to assess model quality with consensus to improve performance (Ray et al., 2012). Thus, it is no surprise that it outperforms the pure consensus methods, like Bipart and ModFOLDclust2. However, by constructing a hybrid method from Bipart similar to Pcombdomain (Elofsson et al., 2017) it should be possible to improve performance further. But that is not 16 the focus of the current study.

217 Final remarks

218 There should be cases where Bipart splits one of two or more domains into half, since the method is deviced to always split the method into two domains. In some cases the domain boundary ought to be in an environment not detectable using the simplistic bipart method, which uses smoothing operators and heuristics to make the domains less choppy segmentially and more even in sizes. Also, when there are more domains than two, the ansatz to use only two partitions may be the cause of any boundary errors.

The difference between Vanilla and Bipart in Figure 4a emphasize that the main drawbacks of 25 the Bipart method is not only that it is simply a Bipart, with no choice on whether to partition at all or partition more; it is also due to that the loss of information of the partitioning in the global score is not mitigated by a propagation of an estimate of these effects through the consideration of the partition interfaces. If one would allow for more than two partitions, the detrimental effect of loosing information upon partitioning would potentially be even greater, and the possible gains on the lower end of the correlation spectrum (from allowing more local and precise superpositions) would get further displaced by the losses on the main part of the upper distribution due to the greater loss of global information, see Figure $4 a$.

The difference between a pure contact map based measure, like LDDT, and our Bipart method (or a partition method with a deeper hierarchy) is that it considers the global relationships through keeping a subset of superpositions. These superpositions gives some global information within the hierarchy and relationships of the partitions; but it does not utilize the full potential, since it does not consider the interfaces between the partitions in formulating the global score (and local scores). A method that uses both the hierarchical information to translate the local errors in the interfaces 239 to the global scores (and somewhat to the local scores of interfacing residues) and measure the 240 local scores in a local fashion (i.e. within partitions) is expected to perform much more balanced 241 with regard to its global and local correlations to gold standard measures. It would be wise to 242 try adress the local-to-global error propagation first, before trying improving the method upon a 243 deeper hierarchy of domain partitions since that would, in the light of previous discussion, only 244 promise to improve the local scores and be detrimental to the global score. 


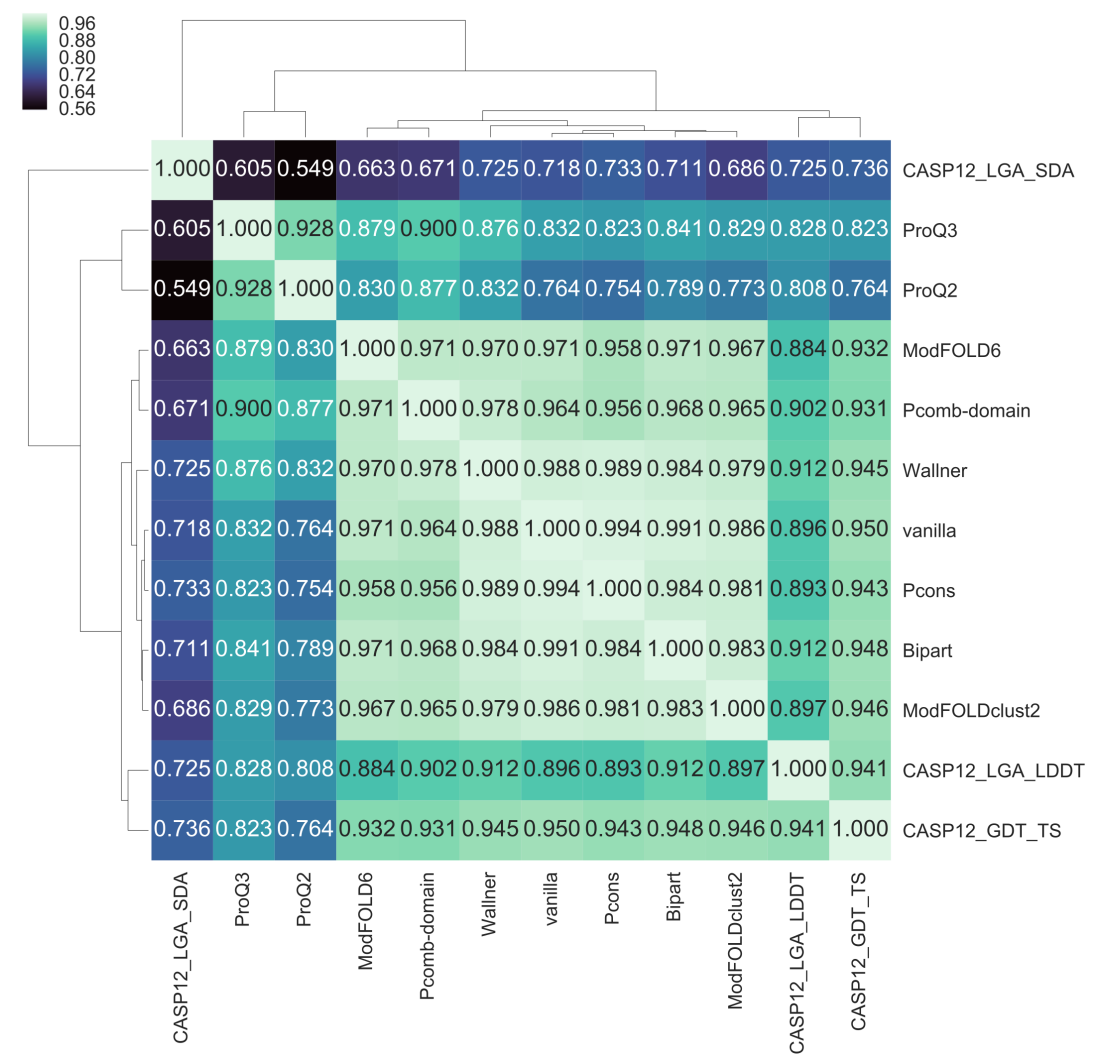

Figure 8. Correlations for global scores for top-performing methods in CASP12.

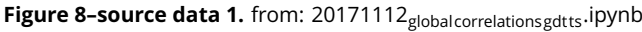



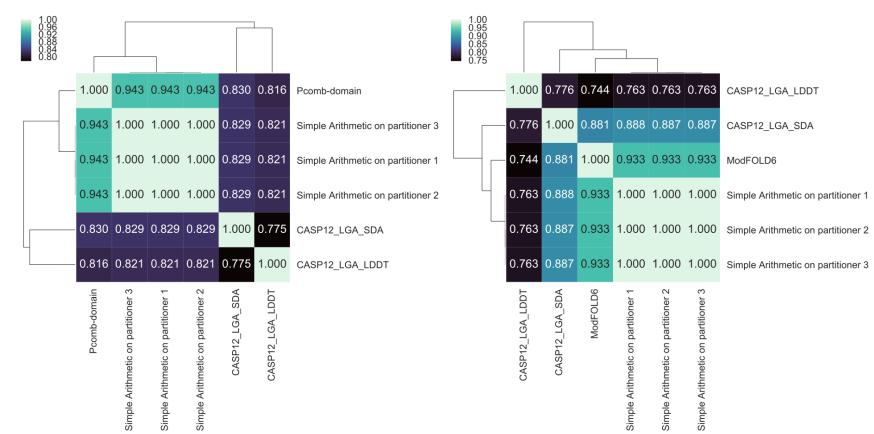

(a) Pcomb-domain

(b) ModFOLD6

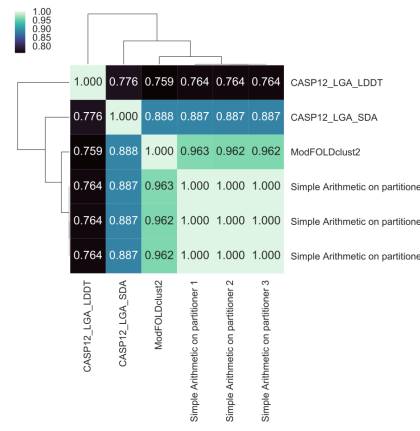

(c) ModFOLDclust2

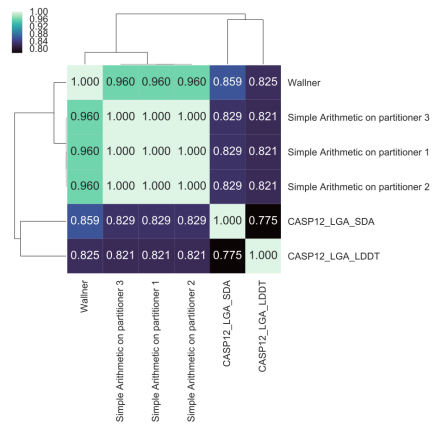

(d) Wallner

Figure 9. Pairwise comparisons between Bipart methods and selected top-performing methods from CASP12 using correlations for local scores.

Figure 9-source data 1. from: $20171112_{\text {detailedcorrelations. }}$.jpynb 
245 Discussion

246 That fact that we see partitioned Pcons (Bipart) fare better on LDDT, the superposition independent 247 measure, is to be expected since partitioning gives us multiple superpositions due to the subse48 quent Pcons run on either of the partitions (Mariani et al., 2017). This makes the Pcons-partition 24 methodology less sensitive to domain orientations changes in its power to discern local model quality.

When performing the local superpositions in Bipart, we loose some global information due to the contacts beeing severed in the partitioning. These are preserved in a global superposition, although we therein risk garbling information on the local level due to the sensitivity to domain orientation. Therefore, it is of interest to develop a hierarchical measure that is sensitive to both the global orientation ("quarternary" domain packing) in the global score and at the same time reliable on the local level (domain integrity). Such a method is capable of correctly propagating errors in interfaces of larger structural units to the global score while at the same time maintaining adequate accuracy in assessing the local scores on the domain levels. Therefore it seems that pivotal points should influence the global score more than other less pivotal areas, while local scores can be assessed using local scoring methods such as LDDT or similar.

We can see that Pcons-partition is a method considering both global and local qualities, as the correlation between LDDT and LGA is significantly lower than that of Pcons-partition and LGA/LDDT.

A reason to why vanilla Pcons performs markedly better when compared to LGA (S-score9, with respect to LDDT, could be due to its usage of multiple superpositions (all vs all) when deciding optimum distance measure for the local scores; this makes vanilla Pcons sort of a pseudo local algorithm, as compared to the purely contact based LDDT.

One of the motivational points of this study was to corroborate or falsify whether this kind of domain defintions are helpful for studying protein structures in the sense that they can make the assessment of correct protein structures more accurate. This would then reflect that these domains give more discriminative power over the assessment of functional protein forms, which means that they serve to partition the protein structures into domain units that constitute or reflect some form of biological relevance or function; why would they otherwise improve the result on quality assessment more than another arbitrary partitioning procedure?

It can be noted the current implementation of Bipart takes for granted that we actually want to cluster the decoys and identify a consensus; but is this always true, do we really want a the most common answer? What if it is wrong? By instead posing the problem from the start, and working from bottom up again, we might find another interesting observable that might be an even better predictor than the simple "all can't be wrong" paradigm.

There are other fields on where the same clustering paradigm as employed here can be useful; one such are in the field of evolutionary coupling analysis, where identification of smaller domains can be used to divide larger problems, and possibly synthesize data from multiple sources. Since these methods produce matrices much like the similarity matrices used here, the use case is almost seamlessly identical.

Since the local attribution of GDT_TS (the LGA measure) is signalling domain alignment errors the most in the residues the furthest away from the actual erronous domain interfaces, it should not be regarded as a true local score. This is because, in general, the error get increasingly worse, due to trigonometry, the further away from the domain interface alignment error it gets. Due to the erronous error propagation of GDT_TS on the local level, effort should be invested in developing a local-to-global scoring scheme that is context aware. Such a scoring scheme could potentially help alleviating the need to either sacrifice global or local accuracy in scoring models.

Correlations with global scores, to a model measure such as GDT_TS, should somewhat reflect model functionality score; since this takes into account all facets of the structure at the same time. It is worth to note though, that such a measure has no knowledge of contexts, it would for an example score an interesting pocket formation as important as a long non-rigid protruding 
loop which obviously would have multiple conformations in reality and therefore the particular conformation represented in the gold standard model would be of relatively minor importance.

In general, it seems safe to perform a partition on a per target basis, as the risks of severe losses are slim while there are chances of greater gains. In most cases where Vanilla is better, we have a runner-up that performs almost equally well on the global correlations of Bipart. On the same 300 time, when Vanilla is worse than Bipart, it tends to be this by a larger margin. The cases where 301 Vanilla is better are more plentiful, with the cases where there are more gain on the Bipart side of correlations being more scarse. Therefore we get the situation where the risk of severe loss on correlative accuracy is not big on a per target basis, while there is a smaller but existent chance 304 of greater gains upon partitioning. It would be interesting to see if the targets with small losses 305 comprise targets of single domain, or more rigid domain proteins, while the those with great gains are multidomain and configuration flexible proteins.

\section{Further Work}

308 A pivotal problem for domain partitioning is the stopping criteria, i.e. deciding if and when partis. tioning is finished. Suggestions would be to use the different QA measures to gauge the partition 310 sanity. Further one could go so far as to use the measures as the relation inducing the partitions, 11 by empoloying Pcons or similar methods to define the partitions. Another obvious improvement, 2 working incrementally from the Bipart method, would be to implement criteria for deciding on how many domains (i.e. clusters) to partition into. As it is now, it always partitions into two domains, while improved flexibility and potentially performance could be possible by dropping this restraint.

Structural bias is a problem discussed but not fully tackled in the present work; here we simply ignore comparisions of decoys generated by the same mathematical model (i.e. server name). In the long run one would ideally want a $M_{\text {eff }}$ (Morcos et al., 2011) for structures, which could be conceived in a variety of ways, initially using a simple RMSD measure based on the Kabsch algorithm. Other more elaborate clustering schemes can be conceived. Furthermore, doing it for each putative partition would also help differentiate in the structural conservation over different regions of each decoy.

For partitioning there might be other approaches that can be even more applicable than spectral clustering for our problem of breaking up protein structures into smaller domains. A data structure that could be analyzed in such a endevaour would be any detectable patterns in the evolution of similarity matrices, using different parameter settings for any set of different similarity functions. Here, it might be possible to detect measurables that are able to distinguish clusters more efficiently than simply spectral clustering. Otherwise, it is also an avenue for producing a tensor for a tensor version of spectral clustering.

An interesting avenue for another kind of domain partitioning algorithm would be to build a tree structure from measuring model quality using many different superpositions. In its simple form, it could be implemented using a K-mer window, superposing only that K-mer and evaluating the resulting scores. Then by analyzing this tree structure, particular residues in the protein that change the score-tree upon being within or outside of the K-mer selection can be identified and used as potential partition points. Otherwise, a score could be developed from such a structure of measures itself.

This idea of divide and conquer into domain partitions can be used in a superposition free methodology based upon contact maps, or similarity matrices. Here, it could serve as to construct a hierarchy within which the QA can be gauged on different levels, enabling quality differentiation by upweighing of errors in pivotal and or structural important regions. By investigating the distribution of hubs and domain interfaces in a protein ensemble structure, context weighted scores could be developed so that an interfacial and hub internal error gets upwheighed on a global scores. 
342 Methods and Materials

343 Dataset

344 To the develop and benchmark the method, models submitted to CASP12 (Moult et al., 2018), were

345 used. All predicted models along with their calculated global and local quality measures were

346 downloaded from the Prediction Center (http://predictioncenter.org/download_area/CASP12/),

347 and stored in a a sqlite3 database outlined below (see Database design section).

348 Pcons

349 Pcons (Wallner and Elofsson, 2005) is a protein model quality assessment program that is using an so ensemble of models to predict both the local and global quality using consensus. The prediction

351 is obtained be superimposing all models in the ensemble pairwise using the similarity measure

352 S-score (Cristobal et al., 2001), see Eq:1. Each model is given a local score corresponding to the

353 local similarity to all other models in the ensemble, i.e average $<S_{i}>$ in Eq:1, and a global score

354 corresponding to $\sum\left\langle S_{i}>\right.$ over the whole sequence. Despite its simplicity Pcons has been one

355 of the top-performing methods since its introduction in CASP5 (Wallner et al., 2003; Wallner and

356 Elofsson, 2007; Larsson et al., 2009; Elofsson et al., 2017).

$$
S_{i}=\frac{1}{1+\left(d_{i} / d_{o}\right)^{2}}
$$

357 where $d_{i}$ is the local distance deviation in of residue $i$, after an optimal superposition that maximizes $358 \sum S_{i}$ over the whole sequence, $d_{0}$ is a distance cutoff set to $3 \AA$.

\section{Domain partitioner}

360 Ensemble models of targets were downloaded from predictioncenter.org (Figure 1a). For each

361 target, the largest subset of models with the same number of residues present in the model files

362 was selected by a bash-script (Figure $1 \mathrm{~b}$, reference the appendix table). These models were then

363 processed into a tensor by extracting a distance matrix using a Python script and output it as a

364 MATLAB array (Figure 1c).

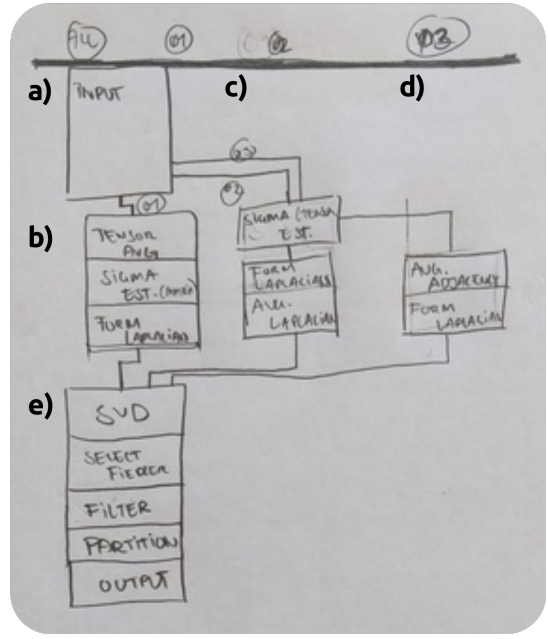

Figure 10. Detailed outline of Bipart algorithm.

Figure 10-source data 1. from: 2017-07-17 casp12 method sketches 


\begin{tabular}{|c|c|c|}
\hline Table & Attributes & Comment \\
\hline Target & ID, Casp.ID, Path.Pathway, Slen & \\
\hline Domain & ID, Method.ID & Segments and domain size in a separate view \\
\hline Component & ID, Target.ID, num, Domain.ID & \\
\hline Segment & Domain.ID, Start, Stop, len & \\
\hline Casp & ID, Path.Pathway & \\
\hline \multirow[t]{2}{*}{ Method } & ID, Name, Description, Type & Type takes four values; \\
\hline & & Server, Partitioner, Compounder, QA \\
\hline \multirow[t]{2}{*}{ Model } & $\begin{array}{l}\text { ID, Method.ID, Target.ID, } \\
\text { Path.Pathway, Name }\end{array}$ & $\begin{array}{l}\text { Name is model running name, } \\
\text { ex. in *_TS1 we have TS1. }\end{array}$ \\
\hline & & $\begin{array}{l}\text { (Method, Target, Name) are unique and } \\
\text { Path is unique in its own right }\end{array}$ \\
\hline \multirow[t]{3}{*}{ QA } & $\begin{array}{l}\text { ID, Model.ID, Component.ID, } \\
\text { Method.ID }\end{array}$ & $\begin{array}{l}\text { if Component.ID is NULL, } \\
\text { the quality is on full model }\end{array}$ \\
\hline & & $\begin{array}{l}\text { Method.ID is pointing to a QA-method or a } \\
\text { compounded QA-method }\end{array}$ \\
\hline & & Model, Component and Method are UNIQUE \\
\hline QAscore & qa.ID, global, local & local should be a parseable text array \\
\hline QAjoin & QA.ID, QA.ID & $\begin{array}{l}\text { From.ID, To.ID, where to is the compounded; } \\
\text { defines which QA's that are compounded. }\end{array}$ \\
\hline Path & Pathway & Directory or file, in text. \\
\hline
\end{tabular}

Table 2. Database tables

The first step in the BiPART algoirthm is to read in the distance tensor as an array and the global quality assessment as a vector from file (Figure 10a). Next the algorithm first collapse the tensor 367 into a square matrix, then estimates a similarity matrix formation sigma and forms the Laplacian 368 matrix (Figure 10b). There were two other variants developed; one method that estimates the sigma 369 over each distance matrix and forms a separate Laplacian which is then averaged (Figure 10c), and 370 another method that averages over the adjacency matrix and then forms the Laplacian (Figure 10d). 371 All collapses of the tensor to a matrix by averaging is done over the tensors model dimension, using 372 the global quality assessment vector as weights. Thereafter all variants follow the same scheme as 373 in von Luxburg (2007) by performing the singular value decomposition, identifying the vector with 37 smallest non-zero singular value as the fiedler vector, applying sequential and de-noising filtering 5 and partitioning using t-test (Figure $10 \mathrm{e}$ ). The result is then output in a text file as a boolean vector with residue pertaining to the first domain as zeroes and the other domain as ones.

After the BIPART algorithm has produced its domain defintions, the PDB MASKER reads this definitions, figures out the corresponding residues and writes a Pcons residue ignore file for each domain definition (Figure 1d). These residue masks can be used to define two ensembles of models, one pertaining to each domain defined by BiPART.

Pcons (Wallner and Elofsson, 2005) is then executed using the functionality to ignore residues 2 from the structural superposition. Each of the ignore masks generated by BiPART are used to 3 generate local quality assessment for each of the domains separately. The resulting local quality 384 assessment matrices are then concatenated, forming a new global quality score for the full models 385 using the $\Sigma<S_{i}>$ as described above in the same way as the regular Pcons (Figure $1 \mathrm{f}$ ).

386 Database design

387 All results, QA and domain definitions are stored in a sqlite3 database with tables as outline in

388 Table 2 . 


\section{References}

Berman HM, Westbrook J, Feng Z, Gilliland G, Bhat TN, Weissig H, Shindyalov IN, Bourne PE. The Protein Data Bank. Nucleic acids research. 2000 Jan; 28(1):235-242.

Buenavista MT, Roche DB, McGuffin LJ. Improvement of 3D protein models using multiple templates guided by single-template model quality assessment. Bioinformatics (Oxford, England). 2012 Jul; 28(14):1851-1857.

4 Cristobal S, Zemla A, Fischer D, Rychlewski L, Elofsson A. A study of quality measures for protein threading models. BMC bioinformatics. 2001; 2(5).

DeLano WL. The PyMOL Molecular Graphics System. wwwpymolorg. 2002;

Elofsson A, Joo K, Keasar C, Lee J, Maghrabi AHA, Manavalan B, McGuffin LJ, Ménendez Hurtado D, Mirabello C, Pilstål R, Sidi T, Uziela K, Wallner B. Methods for Estimation of Model Accuracy in CASP12. Proteins. 2017; doi: 10.1002/prot.25395.

Ginalski K, Elofsson A, Fischer D, Rychlewski L. 3D-Jury: A Simple Approach to Improve Protein Structure Predictions. Bioinformatics. 2017; 19(8):1015-1018. https://academic.oup.com/bioinformatics/article/19/8/ 1015/235399/3D-Jury-a-simple-approach-to-improve-protein, doi: 10.1093/bioinformatics/btg124.

Kryshtafovych A, Monastyrskyy B, Fidelis K, Schwede T, Tramontano A. Assessment of model accuracy estimations in CASP12. Proteins. 2018 Mar; 86 Suppl 1:345-360.

Larsson P, Skwark MJ, Wallner B, Elofsson A. Assessment of global and local model quality in CASP8 using Pcons and ProQ. Proteins: Structure, Function, and Bioinformatics. 2009; 77 Suppl 9(S9):167-172.

Levitt M, Gerstein M. A Unified Statistical Framework for Sequence Comparison and Structure Comparison. Proceedings of the National Academy of Sciences. 2017: 95(11):5913-5920. http://www.pnas.org/content/95/ $11 / 5913$.

von Luxburg U. A Tutorial on Spectral Clustering. CoRR. 2007; abs/0711.0189. http://arxiv.org/abs/0711.0189.

Manavalan B, Lee J. SVMQA: support-vector-machine-based protein single-model quality assessment. Bioinformatics (Oxford, England). 2017 Aug; 33(16):2496-2503.

Mariani V, Biasini M, Barbato A, Schwede T. IDDT: A Local Superposition-Free Score for Comparing Protein Structures and Models Using Distance Difference Tests. Bioinformatics. 2017; 29(21):2722-2728. https:// academic.oup.com/bioinformatics/article/29/21/2722/195896/IDDT-a-local-superposition-free-score-for, doi: 10.1093/bioinformatics/btt473.

McGuffin LJ, Roche DB. Rapid model quality assessment for protein structure predictions using the comparison of multiple models without structural alignments. Bioinformatics (Oxford, England). 2010 Jan; 26(2):182-188.

Morcos F, Pagnani A, Lunt B, Bertolino A, Marks DS, Sander C, Zecchina R, Onuchic JN, Hwa T, Weigt M. DirectCoupling Analysis of Residue Coevolution Captures Native Contacts across Many Protein Families. Proceedings of the National Academy of Sciences of the United States of America. 2011; 108(49):E1293-301. http: //www.pubmedcentral.nih.gov/articlerender.fcgi?artid=3241805\&tool=pmcentrez\&rendertype=abstract, doi: 10.1073/pnas.1111471108

Moult J, Fidelis K, Kryshtafovych A, Schwede T, Tramontano A. Critical assessment of methods of protein structure prediction (CASP)-Round XII. Proteins. 2018 Mar; 86 Suppl 1:7-15.

Olechnovič K, Kulberkytè E, Venclovas C. CAD-score: a new contact area difference-based function for evaluation of protein structural models. Proteins. 2013 Jan; 81(1):149-162.

Ray A, Lindahl E, Wallner B. Improved model quality assessment using ProQ2. BMC bioinformatics. 2012; 13(1):224.

Siew N, Elofsson A, Rychlewski L, Fischer D. MaxSub: An automated measure to assess the quality of protein structure predictions. Bionformatics. 2000; 16(9):776-785.

Skwark MJ, Elofsson A. PconsD: ultra rapid, accurate model quality assessment for protein structure prediction. Bioinformatics (Oxford, England). 2013 Jul; 29(14):1817-1818.

434 The UniProt Consortium. UniProt: the universal protein knowledgebase. Nucleic acids research. 2017 Jan; 45(D1):D158-D169. 
436 Uziela K, Menendez Hurtado D, Shu N, Wallner B, Elofsson A. ProQ3D: improved model quality assessments 437 using deep learning. Bioinformatics (Oxford, England). 2017; 33(10):1578-1580.

438 Uziela K, Wallner B. ProQ2: estimation of model accuracy implemented in Rosetta. Bioinformatics (Oxford, $439 \quad$ England). 2016; 32(9):1411-1413.

440 Wallner B, Elofsson A. Pcons5: combining consensus, structural evaluation and fold recognition scores. $441 \quad$ Bioinformatics (Oxford, England). 2005 Dec; 21(23):4248-4254.

442 Wallner B, Elofsson A. Prediction of global and local model quality in CASP7 using Pcons and ProQ. Proteins: 443 Structure, Function, and Bioinformatics. 2007; 69(S8):184-193.

444 Wallner B, Fang H, Elofsson A. Automatic consensus-based fold recognition using Pcons, ProQ, and Pmodeller. 445 Proteins: Structure, Function, and Bioinformatics. 2003; 53 Suppl 6(S6):534-541.

446 Zemla A, Venclovas C, Moult J, Fidelis K. Processing and analysis of CASP3 protein structure predictions. Proteins: 447 Structure, Function, and Bioinformatics. 1999; Suppl 3:22-29.

448 Zhang Y, Skolnick J. Scoring function for automated assessment of protein structure template quality. Proteins: 449 Structure, Function, and Bioinformatics. 2004 Dec; 57(4):702-710. 



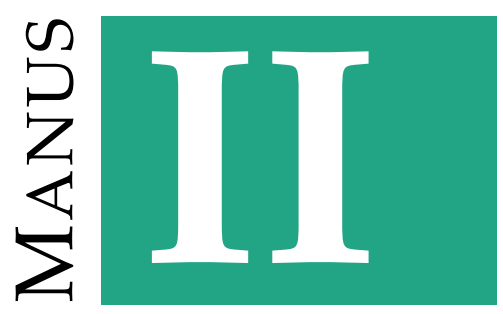

Robert Pilstål, Maria Sunnerhagen, and Björn Wallner. "Functional Interaction Network of c-MYC Conserved Regions determined by Evolutionary Couplings". Apr. 2018 



\section{CMBI: Functional Interaction} Network of c-MYC Conserved Regions determined by Evolutionary Couplings.

*For correspondence: robert.pilstal@liu.se (KRP); bjorn.wallner@liu.se (BW) Present address: ${ }^{\mathrm{I}} \mathrm{FM}$, Linköpings universitet, SE-581 83 Linköping

\author{
Karl Robert Pilstål ${ }^{1 *}$, Maria Sunnerhagen $^{2 \S}$, Björn Wallner $^{1 \star \S}$ \\ ${ }^{1}$ Department of Physics, Chemistry and Biology - Division of Bioinformatics; ${ }^{2}$ Department \\ of Physics, Chemistry and Biology - Division of Chemistry
}

9 Abstract The c-MYC protein is powerful oncoprotein and a major regulator of translation of 10 entire gene programs in cell cycle regulation. It is deregegulated and overexpressed in more than $150 \%$ of cancers. The deregulation of MYC is associated with poor prognosis and aggressive disease, 12 thus developing therapeutics that would inhibit MYC is crucial. But in order to develop MYC

3 inhibitors a greater understanding of the mechanism that regulate MYC expression and activity are 14 needed. Much of the regulation mechanism involves intricate networks of protein-protein

5 interactions and post-translational modifications (PTMs) that modulate the activity. A central part of the regulation is coordinated by the regions of interactions called Myc-Boxes (MB), well conserved regions within the Myc proteins and directly responsible for the interaction with a large number of protein. The Myc-Boxes are located in an disordered part the protein making them extremely difficult to characterize experimentally. Here, we apply the latest computational evolutionary coupling analysis to gain some additional understanding of the human Myc protein. We analyze both the individual Myc-Boxes and pairwise interactions between boxes. We find several residues within and between Myc-Boxes that are coupled, many of which are known to be important for the regulation of Myc, e.g phosphorylation sites T58, S62, as well as sites regulated through 24 post-translationally modification, K52. We also suggest a specific novel interaction between MB1-MB2 involving the residues T58 in MB1 and I130, Q131 in MB2.

\section{Introduction}

28 The c-MYC (MYC) protein (439 aa) is a powerful oncoprotein and a major regulator of translation of entire gene programs in cell cycle regulation. It is deregegulated and overexpressed in more so than 50\% of cancers (Nesbit et al., 1999; Meyer and Penn, 2008; Dang, 2012; Kalkat et al., 2017). The deregulation of MYC is associated with poor prognosis and aggressive disease(McKeown and 32 Bradner, 2014; De Melo et al., 2017), thus developing therapeutics that would inhibit MYC is crucial. 3 But in order to develop MYC inhibitors a greater understanding of the mechanism that regulate 34 MYC expression and activity are needed. Much of the regulation mechanism involves intricate 5 networks of protein-protein interactions and post-translational modifications (PTMs) that modulate the activity Tu et al. (2015).

The structure of MYC consists of a 100-residue C-terminal DNA binding bHLH and leucine 38 zipper domain that hetrodimerize with the Max protein, while the remaining 300 aa appears to be 39 disordered, but still interacting with many partners some estimates say as much as $10-15 \%$ of the 


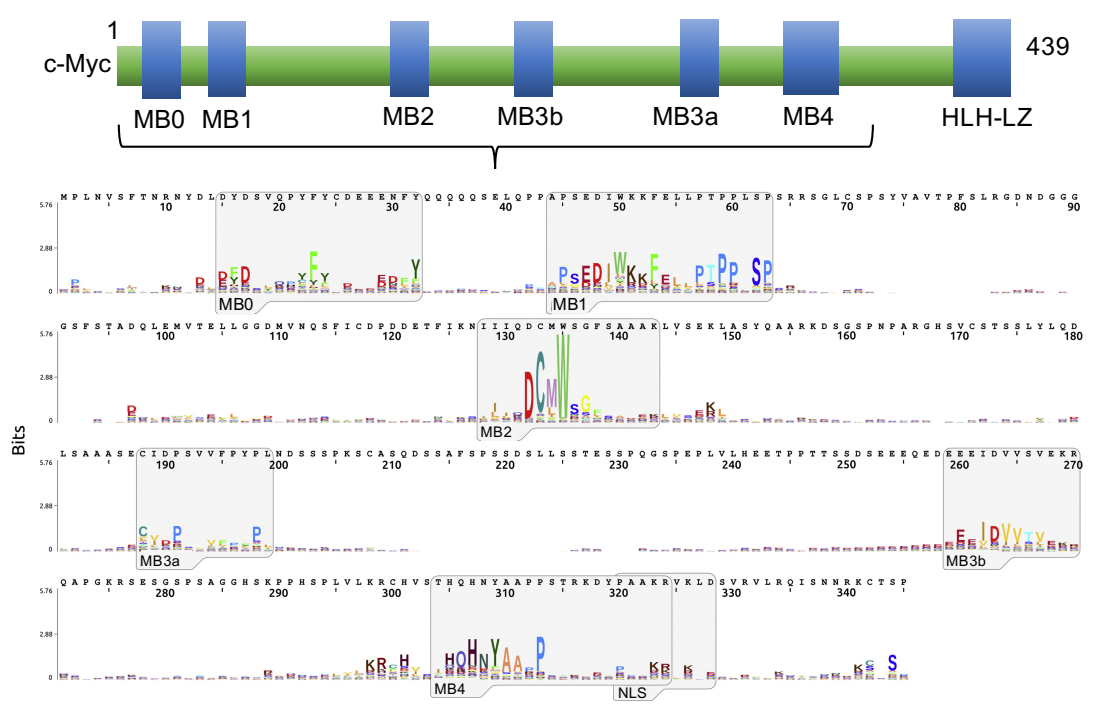

Figure 1. Overview of the Myc interaction regions, i.e Myc boxes (MB) and a sequence logo for the $\mathrm{N}$-terminal part excluding the DNA-binding HLH Leucine Zipper.

40 genome (Meyer and Penn, 2008). Interestingly, the regions of interactions, called Myc-Boxes (MB), 41 are well conserved within Myc proteins and are also directly responsible for the interaction with a 42 large number of proteins (Chan et al., 2014).

Since, disordered proteins are difficult to characterize, it would be extremly useful if compu44 tational methods could be utilized to gain some additional information that can be used design ${ }_{45}$ and direct experiment. Recently, direct coupling analyis of residue coevolution have been shown to

46 capture native contacts and interaction using only sequence information (Morcos et al., 2011; Jones 47 et al., 2012a; Ekeberg et al., 2013) and it has also been applied to disorded proteins (Toth-Petroczy et al., 2016).

Here, we apply the latest coupling analysis to gain some additional understanding of the human 50 Myc protein. We analyze both the individual Myc-Boxes and pairwise interactions between boxes, and validate the predictions on what is known in the litterature.

\section{Results and Discussion}

53 To investigate the interactions within and between the Myc boxes (MB) evolutionary coupling analy${ }_{54}$ sis was performed on boxes visualized in Figure 1 (See Methods). The Myc boxes are conservered sequence motifs in the disordered part N-terminal domain of Myc, with MBIl showing the highest conservation (Figure 1).

Multiple sequence alignments (MSAs) were constructed for the individual and pairwise Myc $s$ boxes, as described in Methods. The effective number of sequences for the individual boxes are shown in Figure 2a. The most conserved Myc box, MB2, is also showing the largest number of $\mathrm{M}_{\text {eff }}$ with around 40 , followed by MB4 and MBO, with 30 and 25, respectively, for the promiscous level. As a rule of thumb $\mathrm{M}_{\text {eff }}$ should be larger than the sequence length to be informative Uones 62 et al., 2015). Also for the pairwise MB interactions, interactions involving MB2 show the largest $6 \mathrm{M}_{\text {eff }}$ values, around 18 (Figure $2 \mathrm{~b}$ ). 


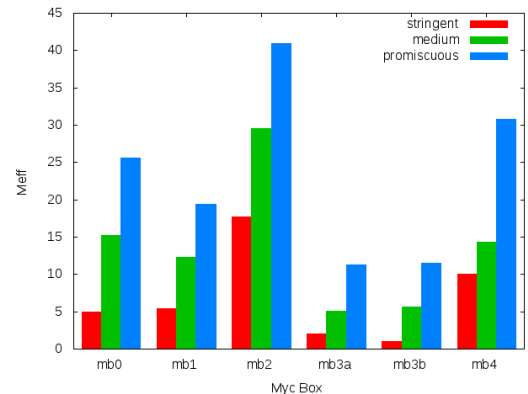

(a)

Figure 2. This histogram shows the effective number of sequences as measured by $\mathrm{M}_{\text {eff }}$ on the vertical axis and boxes considered indicated in on the horizontal axis. $\mathrm{M}_{\text {eff }}$ measures here the variational content for the multiple sequence alignments underlying the box internal interaction predictions for each box. $\mathrm{A}_{e f f}$ as high as the length of the sequence involved is considered good enough to produce reliant predictions in the most significant interactions predicted.

' Figure 2-source data 1. from 20160222_imputation_case_myc_report.pdf

\section{Individual Myc box interactions}

65 The MSAs constructed above for the individual boxes at the promiscous level were used in a direct-

66 coupling analysis using PSICOV (Jones et al., 2012b). The result is presented in Figure 3, highlights

${ }_{67}$ some positions or regions that seem to be evolutionary coupled, in particular, [D15,Y16]-P21,

68 D13-Q20, Y22-[E27,E28], and F23-F31 in MB0 ( $\left.\mathrm{M}_{\text {eff }}=25.6\right)$; F53-T58, L55-S62, P45-[K51,K52] in MB1

$69 \quad\left(\mathrm{M}_{e f f}=19.4\right) ;[\mathrm{F} 138, \mathrm{~S} 139]-\mathrm{L} 144, \mathrm{~S} 139-\mathrm{V} 145$, and F138-F149 in MB2 ( $\left.\mathrm{M}_{\text {eff }}=41.0\right) ; \mathrm{D} 190-\mathrm{F} 195$ in MB3a

$70 \quad\left(\mathrm{M}_{\text {eff }}=11.3\right)$; [Q256,E253]-R270 in MB3b ( $\left.\mathrm{M}_{\text {eff }}=11.5\right)$; and 299R-306Q in MB4 $\left(\mathrm{M}_{\text {eff }}=30.8\right)$. Both

$71 \mathrm{~K} 52$ (De Melo et al., 2017), as well as the region around T58 and S62 as been shown to be hot-spots

72 for cancer-associated mutations (Bahram et al., 2000). It is important to realize that couplings

73 predicted here, might be direct or indirect mediated through a ligand or other partner protein, e.g.

74 positions at the ends of a long helix might be coupled if that helix is interacting with a partner etc.

\section{Pairwise Myc box interactions}

To investigate potential couplings between Myc boxes, the MSAs for the individual boxes were combined as described in Methods, and coupling analysis for pairs of boxes were performed using 8 PSICOV. In this analysis, the intra box interactions are also present, and in almost all cases the strongest couplings are still internal box interactions (Figure 4, Boxes column). Eventough, the internal couplings might have changed compared to the invidual box case above, since they are now analyzed using the combined MSA involving both boxes. Focusing on inter box couplings, given

82 the data at hand, only MB1-MB2 (Figure 4g-h) show a strong coupling through T58-[Q131,1130], and

83 the internal [F138,S139]-L144 is still present in MB2. In the all other cases the interactions involving

84 other boxes are significantly weaker (Figure 4). The interaction between MB1-MB2 is intriguing

85 and has also been reported before Fladvad et al. (2005), but at not at the residue level. Here, we

86 suggest positions (T58-[Q131,1130]) that can be mutated to establish the residues responsible for

87 the interaction

\section{Summary model of the interactions}

89 We have demonstrated that it is possible to extract evolutionary couplings for Myc from sequence

90 data. In order to do this we needed to first split sequences into box entries, or micro domains.

91 Furthermore, when searching for similar sequences, we needed to consider E-value thresholds that 
MBO

$M_{\text {eff }} 25.6$

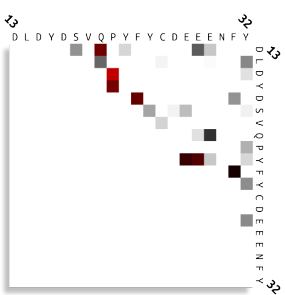

(a) Couplings from PSICOV for MBO

\section{MB1}

$M_{\text {eff }} 19.4$

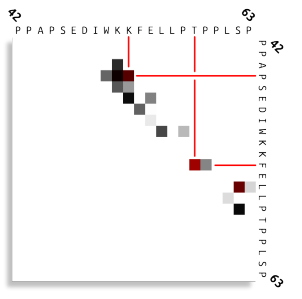

(c) Couplings from PSICOV for MB1.

\section{MB2}

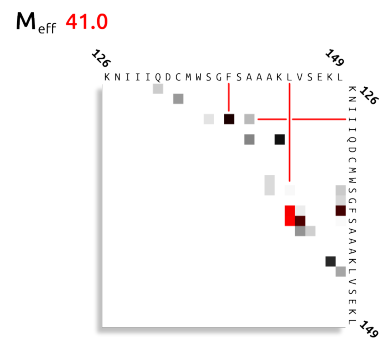

(e) Couplings from PSICOV for MB2.

\begin{tabular}{rrrrrr}
\hline Rank & Pos1 & Res1 & Pos2 & Res2 & Score \\
\hline 1 & 15 & D & 21 & P & 0.88 \\
2 & 16 & Y & 21 & P & 0.73 \\
3 & 13 & D & 20 & Q & 0.72 \\
4 & 17 & D & 23 & F & 0.70 \\
5 & 22 & Y & 28 & E & 0.66 \\
6 & 22 & Y & 27 & E & 0.61 \\
7 & 23 & F & 31 & F & 0.54 \\
8 & 20 & Q & 29 & E & 0.40 \\
9 & 13 & D & 28 & E & 0.32 \\
10 & 14 & L & 20 & Q & 0.30 \\
\hline
\end{tabular}

(b) Top 10 couplings from PSICOV for MBO.

\begin{tabular}{rrrrrr}
\hline Rank & Pos1 & Res1 & Pos2 & Res2 & Score \\
\hline 1 & 53 & $\mathrm{~F}$ & 58 & $\mathrm{~T}$ & 0.81 \\
2 & 55 & $\mathrm{~L}$ & 62 & $\mathrm{~S}$ & 0.71 \\
3 & 45 & $\mathrm{P}$ & 52 & $\mathrm{~K}$ & 0.67 \\
4 & 45 & $\mathrm{P}$ & 51 & $\mathrm{~K}$ & 0.53 \\
5 & 57 & $\mathrm{P}$ & 62 & $\mathrm{~S}$ & 0.48 \\
6 & 47 & $\mathrm{E}$ & 52 & $\mathrm{~K}$ & 0.47 \\
7 & 44 & $\mathrm{~A}$ & 51 & $\mathrm{~K}$ & 0.42 \\
8 & 50 & $\mathrm{~W}$ & 55 & $\mathrm{~L}$ & 0.36 \\
9 & 46 & $\mathrm{~S}$ & 51 & $\mathrm{~K}$ & 0.33 \\
10 & 48 & $\mathrm{D}$ & 53 & $\mathrm{~F}$ & 0.31 \\
\hline
\end{tabular}

(d) Top 10 couplings from PSICOV for MB1.

\begin{tabular}{rrrrrr}
\hline Rank & Pos1 & Res1 & Pos2 & Res2 & Score \\
\hline 1 & 139 & $\mathrm{~S}$ & 144 & $\mathrm{~L}$ & 1.00 \\
2 & 138 & $\mathrm{~F}$ & 144 & $\mathrm{~L}$ & 0.99 \\
3 & 139 & $\mathrm{~S}$ & 145 & $\mathrm{~V}$ & 0.66 \\
4 & 138 & $\mathrm{~F}$ & 149 & $\mathrm{~L}$ & 0.63 \\
5 & 129 & $\mathrm{I}$ & 138 & $\mathrm{~F}$ & 0.56 \\
6 & 131 & $\mathrm{Q}$ & 143 & $\mathrm{~K}$ & 0.47 \\
7 & 143 & $\mathrm{~K}$ & 148 & $\mathrm{~K}$ & 0.42 \\
8 & 131 & $\mathrm{Q}$ & 140 & $\mathrm{~A}$ & 0.24 \\
9 & 140 & $\mathrm{~A}$ & 145 & $\mathrm{~V}$ & 0.21 \\
10 & 127 & $\mathrm{~N}$ & 133 & $\mathrm{C}$ & 0.20 \\
\hline (f) Top 10 couplings from PSICOV for MB2.
\end{tabular}

Figure continues on next page. 


\section{MB3a}

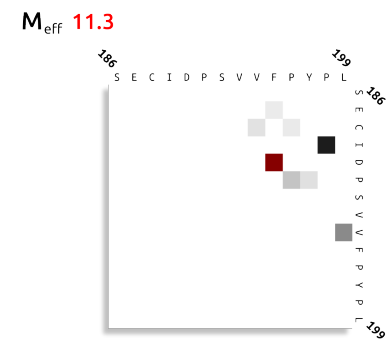

\begin{tabular}{rrrrrr}
\hline Rank & Pos1 & Res1 & Pos2 & Res2 & Score \\
\hline 1 & 190 & $\mathrm{D}$ & 195 & $\mathrm{~F}$ & 0.76 \\
2 & 189 & $\mathrm{I}$ & 198 & $\mathrm{P}$ & 0.45 \\
3 & 194 & $\mathrm{~V}$ & 199 & $\mathrm{~L}$ & 0.23 \\
4 & 191 & $\mathrm{P}$ & 196 & $\mathrm{P}$ & 0.12 \\
5 & 191 & $\mathrm{P}$ & 197 & $\mathrm{Y}$ & 0.06 \\
6 & 188 & $\mathrm{C}$ & 194 & $\mathrm{~V}$ & 0.06 \\
7 & 188 & $\mathrm{C}$ & 196 & $\mathrm{P}$ & 0.04 \\
8 & 187 & $\mathrm{E}$ & 195 & $\mathrm{~F}$ & 0.04 \\
9 & 187 & $\mathrm{E}$ & 196 & $\mathrm{P}$ & -0.05 \\
10 & 191 & $\mathrm{P}$ & 198 & $\mathrm{P}$ & -0.12 \\
\hline
\end{tabular}

(g) Couplings from PSICOV for MB3a.

(h) Top 10 couplings from PSICOV for MB3a.

\section{MB3b}

$M_{\text {eff }} 11.5$

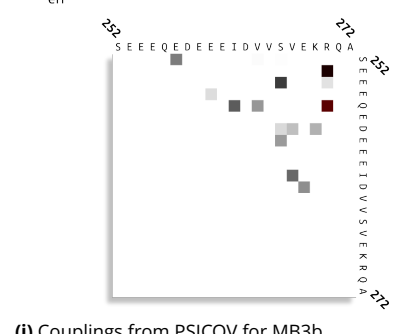

\begin{tabular}{rrrrrr}
\hline Rank & Pos1 & Res1 & Pos2 & Res2 & Score \\
\hline 1 & 256 & Q & 270 & $\mathrm{R}$ & 0.68 \\
2 & 253 & $\mathrm{E}$ & 270 & $\mathrm{R}$ & 0.55 \\
3 & 254 & $\mathrm{E}$ & 266 & $\mathrm{~S}$ & 0.38 \\
4 & 256 & $\mathrm{Q}$ & 262 & $\mathrm{I}$ & 0.32 \\
5 & 262 & $\mathrm{I}$ & 267 & $\mathrm{~V}$ & 0.30 \\
6 & 252 & $\mathrm{~S}$ & 257 & $\mathrm{E}$ & 0.26 \\
7 & 263 & $\mathrm{D}$ & 268 & $\mathrm{E}$ & 0.22 \\
8 & 256 & $\mathrm{Q}$ & 264 & $\mathrm{~V}$ & 0.20 \\
9 & 259 & $\mathrm{E}$ & 266 & $\mathrm{~S}$ & 0.20 \\
10 & 258 & $\mathrm{D}$ & 269 & $\mathrm{~K}$ & 0.15 \\
\hline (j) Top 10 couplings from PSICOV for MB3b.
\end{tabular}

\section{MB4}

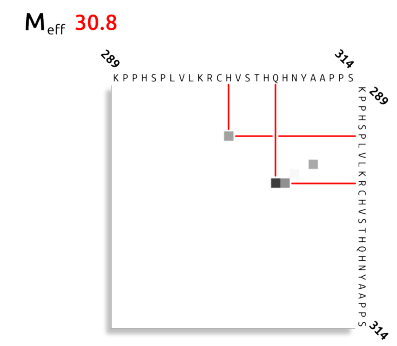

(k) Couplings from PSICOV for MB4

\begin{tabular}{rrrrrr}
\hline Rank & Pos1 & Res1 & Pos2 & Res2 & Score \\
\hline 1 & 299 & $\mathrm{R}$ & 306 & $\mathrm{Q}$ & 0.38 \\
2 & 299 & $\mathrm{R}$ & 307 & $\mathrm{H}$ & 0.22 \\
3 & 294 & $\mathrm{P}$ & 301 & $\mathrm{H}$ & 0.18 \\
4 & 297 & $\mathrm{~L}$ & 310 & $\mathrm{~A}$ & 0.17 \\
5 & 298 & $\mathrm{~K}$ & 308 & $\mathrm{~N}$ & 0.01 \\
6 & 298 & $\mathrm{~K}$ & 311 & $\mathrm{~A}$ & -0.04 \\
7 & 299 & $\mathrm{R}$ & 308 & $\mathrm{~N}$ & -0.05 \\
8 & 307 & $\mathrm{H}$ & 312 & $\mathrm{P}$ & -0.07 \\
9 & 299 & $\mathrm{R}$ & 312 & $\mathrm{P}$ & -0.07 \\
10 & 297 & $\mathrm{~L}$ & 311 & $\mathrm{~A}$ & -0.08 \\
\hline
\end{tabular}

(I) Top 10 couplings from PSICOV for MB4

Figure 3. Covariance coupling using PSICOV for individual boxes. 


\section{$\mathrm{MB0}$ vs $\mathrm{MB1}$}

$\mathrm{M}_{\mathrm{eff}} 4.4$

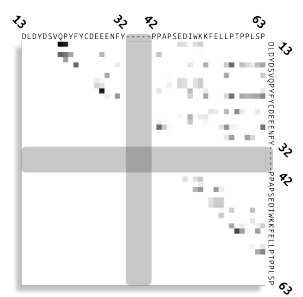

(a) Couplings from PSICOV for MBO-MB1.

\section{MB0 vs MB4}

$M_{\text {eff }} 8.6$

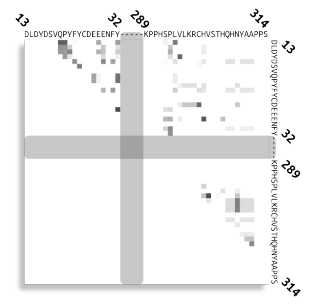

(c) Couplings from PSICOV for MBO-MB4.

\section{$\mathrm{MB0}$ vs $\mathrm{MB3b}$}

$\mathrm{M}_{\mathrm{eff}} \sim 7$

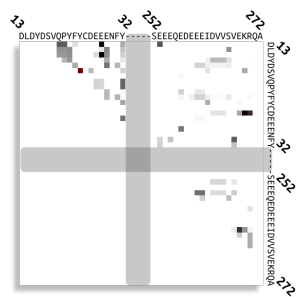

(e) Couplings from PSICOV for MBO-MB3b.

\begin{tabular}{rrrrrrr}
\hline Rank & Pos1 & Res1 & Pos2 & Res2 & Score & Boxes \\
\hline 1 & 13 & D & 20 & Q & 0.49 & MB0 \\
2 & 22 & Y & 28 & E & 0.45 & MB0 \\
3 & 13 & D & 21 & P & 0.44 & MB0 \\
4 & 53 & F & 58 & T & 0.36 & MB1 \\
5 & 15 & D & 20 & Q & 0.30 & MB0 \\
6 & 17 & D & 23 & F & 0.30 & MB0 \\
7 & 17 & D & 57 & P & 0.29 & MB0-MB1 \\
8 & 29 & E & 43 & P & 0.29 & MB0-MB1 \\
9 & 29 & E & 63 & P & 0.28 & MB0-MB1 \\
10 & 23 & F & 57 & P & 0.28 & MB0-MB1 \\
\hline
\end{tabular}

(b) Top 10 couplings from PSICOV for MBO-MB1

\begin{tabular}{rrrrrrr}
\hline Rank & Pos1 & Res1 & Pos2 & Res2 & Score & Boxes \\
\hline 1 & 27 & E & 32 & Y & 0.35 & MB0 \\
2 & 296 & V & 302 & V & 0.35 & MB4 \\
3 & 29 & E & 301 & H & 0.33 & MBO-MB4 \\
4 & 13 & D & 21 & P & 0.31 & MB0 \\
5 & 13 & D & 20 & Q & 0.31 & MB0 \\
6 & 26 & D & 300 & C & 0.30 & MB0-MB4 \\
7 & 16 & Y & 296 & V & 0.29 & MB0-MB4 \\
8 & 21 & P & 32 & Y & 0.28 & MB0 \\
9 & 20 & Q & 32 & Y & 0.28 & MB0 \\
10 & 13 & D & 295 & L & 0.26 & MB0-MB4 \\
\hline
\end{tabular}

(d) Top 10 couplings from PSICOV for MBO-MB4

\begin{tabular}{rrrrrrr}
\hline Rank & Pos1 & Res1 & Pos2 & Res2 & Score & Boxes \\
\hline 1 & 18 & $\mathrm{~S}$ & 24 & $\mathrm{Y}$ & 0.72 & MB0 \\
2 & 26 & $\mathrm{D}$ & 269 & $\mathrm{~K}$ & 0.54 & MBO-MB3B \\
3 & 13 & $\mathrm{D}$ & 28 & $\mathrm{E}$ & 0.53 & $\mathrm{MBO}$ \\
4 & 15 & $\mathrm{D}$ & 28 & $\mathrm{E}$ & 0.49 & $\mathrm{MBO}$ \\
5 & 26 & $\mathrm{D}$ & 270 & $\mathrm{R}$ & 0.42 & $\mathrm{MBO}-\mathrm{MB} 3 \mathrm{~B}$ \\
6 & 262 & $\mathrm{I}$ & 268 & $\mathrm{E}$ & 0.40 & $\mathrm{MB} 3 \mathrm{~B}$ \\
7 & 13 & $\mathrm{D}$ & 253 & $\mathrm{E}$ & 0.34 & $\mathrm{MBO}-\mathrm{MB} 3 \mathrm{~B}$ \\
8 & 13 & $\mathrm{D}$ & 21 & $\mathrm{P}$ & 0.32 & $\mathrm{MBO}$ \\
9 & 13 & $\mathrm{D}$ & 20 & $\mathrm{Q}$ & 0.32 & $\mathrm{MBO}$ \\
10 & 31 & $\mathrm{~F}$ & 267 & $\mathrm{~V}$ & 0.32 & MBO-MB3B \\
\hline
\end{tabular}

(f) Top 10 couplings from PSICOV for MB0-MB3b. 
MB1 vs MB2

$M_{\text {eff }} 12.6$

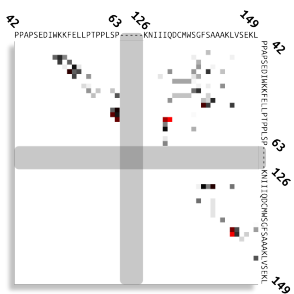

(g) Couplings from PSICOV for MB1-MB2.

\section{$M B 2$ vs $M B 3 b$}

$M_{\text {eff }} 17.3$

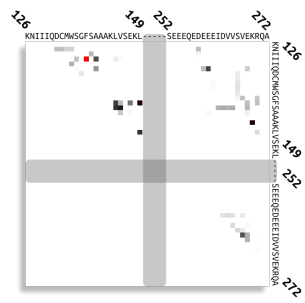

(i) Couplings from PSICOV for MB2-MB3b.

\section{MB2 vs MB4}

$M_{\text {eff }} 18.5$

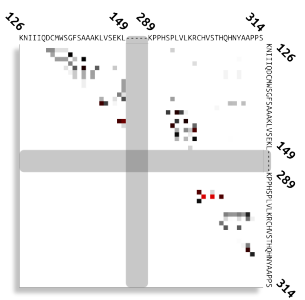

(k) Couplings from PSICOV for MB2-MB4.

\begin{tabular}{rrrrrrr}
\hline Rank & Pos1 & Res1 & Pos2 & Res2 & Score & Boxes \\
\hline 1 & 58 & T & 131 & $\mathrm{Q}$ & 1.00 & MB1-MB2 \\
2 & 139 & $\mathrm{~S}$ & 144 & $\mathrm{~L}$ & 0.98 & MB2 \\
3 & 58 & $\mathrm{~T}$ & 130 & $\mathrm{I}$ & 0.83 & MB1-MB2 \\
4 & 138 & $\mathrm{~F}$ & 144 & $\mathrm{~L}$ & 0.73 & MB2 \\
5 & 58 & $\mathrm{~T}$ & 63 & $\mathrm{P}$ & 0.70 & $\mathrm{MB1}$ \\
6 & 57 & $\mathrm{P}$ & 62 & $\mathrm{~S}$ & 0.69 & $\mathrm{MB} 1$ \\
7 & 55 & $\mathrm{~L}$ & 138 & $\mathrm{~F}$ & 0.64 & MB1-MB2 \\
8 & 48 & $\mathrm{D}$ & 53 & $\mathrm{~F}$ & 0.59 & $\mathrm{MB1}$ \\
9 & 129 & $\mathrm{I}$ & 140 & $\mathrm{~A}$ & 0.57 & $\mathrm{MB2}$ \\
10 & 56 & $\mathrm{~L}$ & 63 & $\mathrm{P}$ & 0.54 & $\mathrm{MB1}$ \\
\hline
\end{tabular}

(h) Top 10 couplings from PSICOV for MB1-MB2

\begin{tabular}{rrrrrrr}
\hline Rank & Pos1 & Res1 & Pos2 & Res2 & Score & Boxes \\
\hline 1 & 129 & $\mathrm{I}$ & 138 & $\mathrm{~F}$ & 1.11 & $\mathrm{MB} 2$ \\
2 & 142 & $\mathrm{~A}$ & 269 & $\mathrm{~K}$ & 0.55 & MB2-MB3B \\
3 & 138 & $\mathrm{~F}$ & 149 & $\mathrm{~L}$ & 0.55 & $\mathrm{MB2}$ \\
4 & 139 & $\mathrm{~S}$ & 145 & $\mathrm{~V}$ & 0.44 & $\mathrm{MB2}$ \\
5 & 139 & $\mathrm{~S}$ & 144 & $\mathrm{~L}$ & 0.41 & $\mathrm{MB2}$ \\
6 & 144 & $\mathrm{~L}$ & 149 & $\mathrm{~L}$ & 0.41 & $\mathrm{MB} 2$ \\
7 & 138 & $\mathrm{~F}$ & 144 & $\mathrm{~L}$ & 0.38 & $\mathrm{MB} 2$ \\
8 & 131 & $\mathrm{Q}$ & 260 & $\mathrm{E}$ & 0.37 & MB2-MB3B \\
9 & 129 & $\mathrm{I}$ & 140 & $\mathrm{~A}$ & 0.35 & $\mathrm{MB2}$ \\
10 & 262 & $\mathrm{I}$ & 267 & $\mathrm{~V}$ & 0.35 & MB3B \\
\hline (j) Top 10 couplings from PSICOV for MB2-MB3b.
\end{tabular}

\begin{tabular}{rrrrrrr}
\hline Rank & Pos1 & Res1 & Pos2 & Res2 & Score & Boxes \\
\hline 1 & 294 & $\mathrm{P}$ & 303 & $\mathrm{~S}$ & 0.93 & MB4 \\
2 & 294 & $\mathrm{P}$ & 301 & $\mathrm{H}$ & 0.92 & MB4 \\
3 & 143 & $\mathrm{~K}$ & 149 & $\mathrm{~L}$ & 0.72 & MB2 \\
4 & 293 & $\mathrm{~S}$ & 300 & $\mathrm{C}$ & 0.66 & MB4 \\
5 & 294 & $\mathrm{P}$ & 305 & $\mathrm{H}$ & 0.65 & $\mathrm{MB} 4$ \\
6 & 145 & $\mathrm{~V}$ & 299 & $\mathrm{R}$ & 0.65 & $\mathrm{MB2}-\mathrm{MB} 4$ \\
7 & 143 & $\mathrm{~K}$ & 148 & $\mathrm{~K}$ & 0.64 & MB2 \\
8 & 139 & $\mathrm{~S}$ & 144 & $\mathrm{~L}$ & 0.62 & MB2 \\
9 & 306 & $\mathrm{Q}$ & 311 & $\mathrm{~A}$ & 0.60 & MB4 \\
10 & 144 & $\mathrm{~L}$ & 294 & $\mathrm{P}$ & 0.60 & MB2-MB4 \\
\hline
\end{tabular}

(I) Top 10 couplings from PSICOV for MB2-MB4. 


\section{MB3a vs MB4}

$\mathrm{M}_{\text {eff }} 9.9$

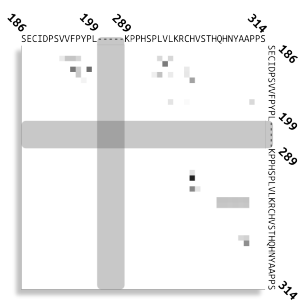

(m) Couplings from PSICOV for MB3a-MB4.

\begin{tabular}{rrrrrrr}
\hline Rank & Pos1 & Res1 & Pos2 & Res2 & Score & Boxes \\
\hline 1 & 294 & $\mathrm{P}$ & 301 & $\mathrm{H}$ & 0.44 & MB4 \\
2 & 190 & $\mathrm{D}$ & 198 & $\mathrm{P}$ & 0.29 & MB3A \\
3 & 190 & $\mathrm{D}$ & 195 & $\mathrm{~F}$ & 0.27 & MB3A \\
4 & 189 & $\mathrm{I}$ & 296 & $\mathrm{~V}$ & 0.26 & MB3A-MB4 \\
5 & 296 & $\mathrm{~V}$ & 301 & $\mathrm{H}$ & 0.23 & MB4 \\
6 & 306 & $\mathrm{Q}$ & 311 & $\mathrm{~A}$ & 0.22 & MB4 \\
7 & 192 & $\mathrm{~S}$ & 301 & $\mathrm{H}$ & 0.18 & MB3A-MB4 \\
8 & 191 & $\mathrm{P}$ & 295 & $\mathrm{~L}$ & 0.12 & MB3A-MB4 \\
9 & 298 & $\mathrm{~K}$ & 311 & $\mathrm{~A}$ & 0.12 & MB4 \\
10 & 298 & $\mathrm{~K}$ & 309 & $\mathrm{Y}$ & 0.12 & MB4 \\
\hline
\end{tabular}

(n) Top 10 couplings from PSICOV for MB3a-MB4.

Figure 4. Covariance coupling using PSICOV for pairs of boxes.

92 traditionally is not considered significant. Unfortunately there are no large data set of structural 3 information for disordered proteins that can be used to conduct a large benchmark. Instead, we choose to validate and motivate the result by comparing to known mutations and interactions for Myc.

An interpretation of the coupling predictions above is presented below; only the box to box interactions that seems to be more consistent between the two box pair MSA generation methods and at the same time present some dominant interactions are included (Figure 5).

The boxes are sequentially connected by a grey curve for ease of interpretation, and the single box interactions are drawn with thick black lines between the boxes. Box pair interaction predictions are shown on the sides and below the central sequence representation. We do find a couple of significant couplings within the boxes, involving mostly [D15,Y16]-P21, Y22-[E27,E28] in MB0; F53T58, L55-S62, P45-[K51,K52] in MB1; [F138,S139]-[L144,V145] in MB2 (corresponding to the red dots in the single box interactions maps). For the pairwise boxes, results the two variants of combining MSAs are shown. Method 1, using only the highest scoring matches for each protein, when combining the box pair MSAs seems to be the most accurate in the sense that it produces 7 more distinct interaction maps. Most of the red dots for the pairs are still within a single box. 8 Only for interactions between MB1 and MB2, the pair interaction mediated by the T58-[Q131,1130] coupling is stronger than the box internal couplings (Figure 5).

\section{Experimental validation}

The most extensively studied pathway regulating MYC stability involves phosphorylation of S62 12 anf T58 residues, followed by isomerization of P61, dephosphorylation of S62 and subsequent 3 ubiquitylation and proteasomal degradation by the E3-ligase, Skp1-Cullin1-Fbox (SCF)Fbxw7 (Yada 14 et al., 2004). Mutations at T58 give rise to highly aggressive tumors (Bahram et al., 2000), due to 115 disturbed proteolytic breakdown as a result of non-functional phosphorylation at this critical site. 16 It is interesting that both phosphorylation sites T58 and S62 in MB1 connects to several residues 7 internal in MB1 (Figure 3c-d and Figure 4g-h) as well as to a region of MB2 $(130,131,144)$, which 18 is critical for transformation. Such correlations may speak both to functional conservation and 119 structural conservation, due to earlier identified interactions between MB1 and MB2 Fladvad et al. (2005).

Besides the two phosorylation sites, $\mathrm{K} 52$ is another residue also showing up as highly coupled to P45 and E47 in MB1 (Figure 3c-d). K52 has been previously been reported to be post-translationally modified both ubiquitylation (Kim et al., 2011) and SUMOylation (González-Prieto et al., 2015) highlighting the importantance of this residue. Recently, a second pathway for MYC regulation involving $\mathrm{K} 52$ was also reported (De Melo et al., 2017).

Similarily, the coupling between residues in the MB0 Pin1-binding region (Helander et al., 2015) 

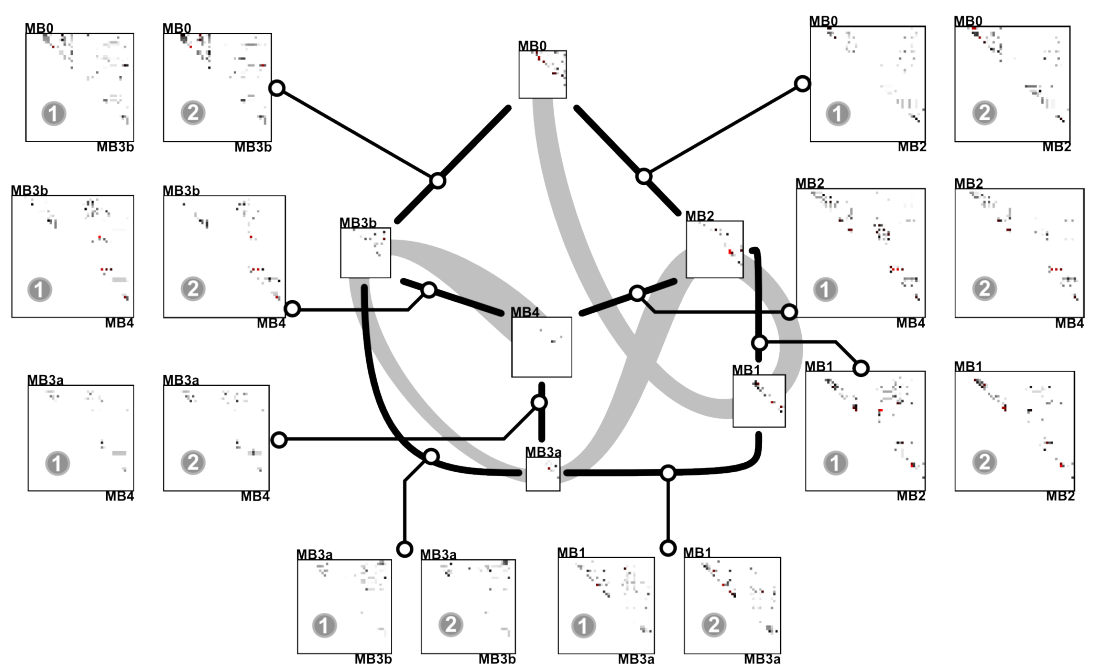

Figure 5. Illustration of the internal and between box coupling interaction predictions. The predictions of the separate boxes internal structures are presented in the central representation of the c-MYC disordered chain in grey, with the interaction predictions using the pairwise MSAs for each pair are illustrated in the margins and their associated box interaction indicated at the central rendering. Each prediction is done with two methods; i.e. 1) only top co-occurences was combined and used, and 2) all co-occurences were combined and used for the pairwise MSAs.

Figure 5-source data 1. from 20160222_imputation_case_myc_report.pdf

$127(17,23,29)$ (Figure 4a-b) with prolines adjacent to the phosphorylated $\mathrm{S} / \mathrm{T}$ residues signalling cis-trans isomerisation by Pin1 (P57, P63) speaks toward a functional coupling: the Pin1-binding anchoring region needs to be conserved in order for cis-trans isomerisation to occur (Liou et al., 2011). Also the coupling within MB0 between 20-23 and 27-31 (Figure 3a-b) agrees nicely with the two touch-points to Pin1: the first (20-23) being the main touch-point to the Pin1 WW domain and the second (27-31) being the main touch-point to the PPlase domain (Helander et al., 2015). Illustrating a case where coupling is achieve by interaction of third partner, Pin1 in this case.

Taken together there is strong support for several of the predicted couplings. The strong coupling between T58 in MB1 and 1130, Q131 in MB2 is novel and would be a perfect target 136 for mutational analysis, something that we are currently persuing. There is also strong coupling between S139-L144 in MB2 that is also worth investigating.

\section{Methods and Materials}

\section{Myc sequence}

The human Myc sequence (P01106, MYC_HUMAN) was downloaded from Uniprot (The UniProt Consortium, 2017). The regulatory myc box regions was defined based on the definitions used in $T u$ et al. (2015) according to Table 1.

\section{Covariance analysis}

44 In order to maximize the use of available data for the modeling of the box interactions, homolo145 gous sequences was searched for each Myc box separately using Jackhmmer (Eddy, 2011) against 146 Uniref100 (Suzek et al, 2015) using three different $\mathrm{E}$-value threshold; from strigent $\mathrm{E}<0.01$, medium

$147 E<1$, and promiscuos $E<10$. The reason to use such large $E$-values is that for short sequences $<30$

148 amino acids it is impossible to get enough hits with E-values at are commonly seen as significant 
Table 1. The myc box definitions used in study.

\begin{tabular}{lrr}
\hline Box & Start & End \\
\hline MB0 & 13 & 32 \\
MBI & 42 & 63 \\
MBII & 126 & 149 \\
MBIIIa & 186 & 199 \\
MBIIIb & 252 & 272 \\
MBIV & 289 & 314 \\
\hline
\end{tabular}

149 even identical matches have relatively high E-values. Still, we are interested in finding the most similar sequences in the database, and by increasing the E-value we will get these sequences. Alternatively, we could have made the filter on the alignment score rather than on the E-value, but that 52 would essentially generate the same ranked list of sequences, and since Jackhmmer only always filtering on E-value this was easier. In the future, the statistics, and multiple testing correction, for 4 short sequences needs to be improved so that we with power can tell which sequences to include. In essence, this problem is related to GWAS studies were it is also hard to find significance because of the multiple testing problem. MSAs.

Finally, PSICOV (Jones et al., 2012b) was used to to predict covariance/contacts for the different

\section{Pairwise covariance analysis}

Pairwise covariance analysis was performed on pairwise MSAs that were constructed by joining sequences on common Uniref100 cluster identifiers. This was performed using two methods that differs in how to pair up multiple matches to the same Uniref100 cluster. The first method (1) choose pairs in the order determined by the highest scoring matches in each protein, removing any any unmatched sequences. The second method (2) combined all pairs of sequences within cluster. Finally, PSICOV (Jones et al., 2012b) was used to to predict covariance/contacts for the two joined MSAs

\section{Effective number of sequence}

To get a measure on the sequence diversity of a MSA, the effective number of sequence, $\mathrm{M}_{e f f}$, is calculated as in Ekeberg et al. (2013). In short, $\mathrm{M}_{\text {eff }}$ is calculated by summing a term corresponding

170 to the inverse of the number of sequence in the MSA with sequence identity above $80 \%$ for each 171 sequence in the MSA Ekeberg et al. (2013). In this way, similar sequences are only counted once.

$72 \mathrm{M}_{\text {eff }}$ was calculated for single and pairwise MSAs, as described above.

173 Further Work

174 Instead of searching directly with the sequences using the Pfam-family entry as in Tu et al. (2015) 175 and start the searches using cut-out HMM-profiles for each box might find more sequences. In 176 addition, searching with other tools such as HHblits might also improve the results. Another way of 177 improving the results could be to impute away the gaps in the MSAs; this was the initial idea, but it 178 was never followed through since the imputation method was in development (unpublished work). 179 Since the statistics are weaker for shorter query searches the box MSAs could be redone increasing 180 the box size to a minimum of 30 residues.

\section{References}

Bahram F, von der Lehr N, Cetinkaya C, Larsson LG. c-Myc hot spot mutations in lymphomas result in inefficient 183 ubiquitination and decreased proteasome-mediated turnover. Blood. 2000 Mar; 95(6):2104-2110. 
Chan PK, Srikumar T, Dingar D, Kalkat M, Penn LZ, Raught B. BioID data of c-MYC interacting protein partners in cultured cells and xenograft tumors. Data in brief. 2014 Dec; 1:76-78.

Dang CV. MYC on the path to cancer. Cell. 2012 Mar; 149(1):22-35.

De Melo J, Kim SS, Lourenco C, Penn LZ. Lysine-52 stabilizes the MYC oncoprotein through an SCFFbxw7independent mechanism. Oncogene. 2017 Dec; 36(49):6815-6822.

Eddy SR. Accelerated Profile HMM Searches. PLoS Computational Biology. 2011 Oct; 7(10):e1002195. http: //dx.plos.org/10.1371/journal.pcbi.1002195, doi: 10.1371/journal.pcbi.1002195.

Ekeberg M, Lövkvist C, Lan Y, Weigt M, Aurell E. Improved contact prediction in proteins: Using pseudolikelihoods to infer Potts models. Physical Review E. 2013 Jan; 87(1):012707-012707. http://link.aps.org/doi/10.1103/ PhysRevE.87.012707, doi: 10.1103/PhysRevE.87.012707.

Fladvad M, Zhou K, Moshref A, Pursglove S, Säfsten P, Sunnerhagen M. N and C-terminal sub-regions in the c-Myc transactivation region and their joint role in creating versatility in folding and binding. Journal of Molecular Biology. 2005 Feb; 346(1):175-189.

González-Prieto R, Cuijpers SA, Kumar R, Hendriks IA, Vertegaal AC. c-Myc is targeted to the proteasome for degradation in a SUMOylation-dependent manner, regulated by PIAS1, SENP7 and RNF4. Cell cycle (Georgetown, Tex). 2015; 14(12):1859-1872.

Helander S, Montecchio M, Pilstål R, Su Y, Kuruvilla J, Elvén M, Ziauddin JM, Anandapadamanaban M, Cristobal S, Lundström P. Pre-Anchoring of Pin1 to Unphosphorylated c-Myc in a Fuzzy Complex Regulates c-Myc Activity. Structure/Folding and Design. 2015; 23(12):2267-2279.

Jones DT, Buchan DWA, Cozzetto D, Pontil M. PSICOV: precise structural contact prediction using sparse inverse covariance estimation on large multiple sequence alignments. Bioinformatics (Oxford, England). 2012 Jan; 28(2):184-190.

Jones DT, Buchan DWa, Cozzetto D, Pontil M. PSICOV: precise structural contact prediction using sparse inverse covariance estimation on large multiple sequence alignments. Bioinformatics (Oxford, England). $2012 \mathrm{Jan}$; 28(2):184-90. http://www.ncbi.nlm.nih.gov/pubmed/22101153, doi: 10.1093/bioinformatics/btr638.

Jones DT, Singh T, Kosciolek T, Tetchner S. MetaPSICOV: combining coevolution methods for accurate prediction of contacts and long range hydrogen bonding in proteins. Bioinformatics (Oxford, England). 2015 Apr; 31(7):999-1006.

Kalkat M, De Melo J, Hickman KA, Lourenco C, Redel C, Resetca D, Tamachi A, Tu WB, Penn LZ. MYC Deregulation in Primary Human Cancers. Genes. 2017 May; 8(6).

Kim W, Bennett EJ, Huttlin EL, Guo A, Li J, Possemato A, Sowa ME, Rad R, Rush J, Comb MJ, Harper JW, Gygi SP. Systematic and quantitative assessment of the ubiquitin-modified proteome. Mol Cell. 2011 Oct; 44(2):325340 .

Liou YC, Zhou XZ, Lu KP. Prolyl isomerase Pin1 as a molecular switch to determine the fate of phosphoproteins. Trends in biochemical sciences. 2011 Oct; 36(10):501-514.

McKeown MR, Bradner JE. Therapeutic Strategies to Inhibit MYC. Cold Spring Harbor Perspectives in Medicine. 2014; 4(10):a014266-a014266.

Meyer N, Penn LZ. Reflecting on 25 years with MYC. Nature reviews Cancer. 2008 Dec; 8(12):976-990.

Morcos F, Pagnani A, Lunt B, Bertolino A, Marks DS, Sander C, Zecchina R, Onuchic JN, Hwa T, Weigt M. Directcoupling analysis of residue coevolution captures native contacts across many protein families. Proceedings of the National Academy of Sciences of the United States of America. 2011 Dec; 108(49):E1293-301.

Nesbit CE, Tersak JM, Prochownik EV. MYC oncogenes and human neoplastic disease. Oncogene. 1999; 18(19):3004-3016.

Suzek BE, Wang Y, Huang H, McGarvey PB, Wu CH. UniRef clusters: a comprehensive and scalable alternative for improving sequence similarity searches. Bioinformatics. 2015 Mar; 31(6):926-932. https://www.ncbi.nIm. nih.gov/pmc/articles/PMC4375400/, doi: 10.1093/bioinformatics/btu739.

The UniProt Consortium. UniProt: the universal protein knowledgebase. Nucleic acids research. 2017 Jan; 45(D1):D158-D169. 
2 Toth-Petroczy A, Palmedo P, Ingraham J, Hopf T, Berger B, Sander C, Marks D. Structured States of Disordered Proteins from Genomic Sequences. Cell. 2016 Sep; 167(1):158-170.e12. http://linkinghub.elsevier.com/ retrieve/pii/S0092867416312430, doi: 10.1016/j.cell.2016.09.010.

Tu WB, Helander S, Pilstål R, Hickman KA, Lourenco C, Jurisica I, Raught B, Wallner B, Sunnerhagen M, Penn LZ. Myc and its interactors take shape. Biochimica et Biophysica Acta (BBA)-Gene Regulatory Mechanisms. 2015; 1849(5):469-483.

Yada M, Hatakeyama S, Kamura T, Nishiyama M, Tsunematsu R, Imaki H, Ishida N, Okumura F, Nakayama K, Nakayama KI. Phosphorylation-dependent degradation of c-Myc is mediated by the F-box protein Fbw7. EMBO J. 2004 May; 23(10):2116-2125. 